\title{
Integrationsfördernde Unternehmensgründung
}

KOMPENDIUM BEWÄHRTER VERFAHREN 



\section{Integrationsfördernde Unternehmensgründung}

KOMPENDIUM BEWÄHRTER VERFAHREN 
Das vorliegende Dokument wird unter der Verantwortung des Generalsekretärs der OECD veröffentlicht. Die darin zum Ausdruck gebrachten Meinungen und Argumente spiegeln nicht zwangsläufig die offizielle Einstellung der OECD-Mitgliedstaaten wider oder die der Europäischen Union wieder.

Dieses Dokument und die darin enthaltenen Karten berühren weder den völkerrechtlichen Status von Territorien noch die Souveränität über Territorien, den Verlauf internationaler Grenzen und Grenzlinien sowie den Namen von Territorien, Städten oder Gebieten.

\section{Bitte zitieren Sie diese Publikation wie folgt:}

OECD/Europäische Union (2016), Die fehlenden Unternehmer 2015: Politiken für selbstständige

Erwerbstätigkeit und Unternehmertum, OECD Publishing.

http://dx.doi.org/10.1787/9789264261402-de

ISBN 978-92-6426138-9 (print)

ISBN 978-92-6426140-2 (PDF)

Europäische Union

ISBN 978-92-79-57969-1 (print)

ISBN 978-92-79-57653-9 (PDF)

Katalognummer

KE-01-16-314-DE-C (print)

KE-01-16-314-DE-N (PDF)

Lettland war zum Zeitpunkt der Erstellung dieser Publikation nicht Mitglied der OECD. Lettland erscheint somit nicht in der Liste der Mitgliedsländer und ist nicht als Teil des OECD-Raums aufgeführt.

Foto(s): Deckblatt @ iStockphoto.com/Kevin Smart

Korrigenda zu OECD-Veröffentlichungen sind verfügbar unter: www.oecd.org/publishing/corrigenda.

() OECD/Europäische Union 2016

Die OECD gestattet das Kopieren, Herunterladen und Abdrucken von OECD-Inhalten für den eigenen Gebrauch sowie das Einfügen von Auszügen aus OECD-Veröffentlichungen, -Datenbanken und -Multimediaprodukten in eigene Dokumente, Präsentationen, Blogs, Websites und Lehrmaterialien, vorausgesetzt die Quelle und der Urheberrechtsinhaber werden in geeigneter Weise genannt. Sämtliche Anfragen bezüglich Verwendung für öffentliche oder kommerzielle Zwecke bzw. Übersetzungsrechte sind zu richten an: rights@oecd.org. Die Genehmigung zur Kopie von Teilen dieser Publikation für den öffentlichen oder kommerziellen Gebrauch ist direkt einzuholen beim Copyright Clearance Center (CCC) unter info@copyright.com oder beim Centre français d'exploitation du droit de copie (CFC) unter contact@cfcopies.com. 


\section{VORWORT}

$\mathrm{D}$ e Stimulation erfolgreicher Unternehmensgründungen über alle Gesellschaftsbereiche hinweg stellt eine wichtige Anforderung dar, um intelligentes, nachhaltiges und integratives Wachstum zu erreichen und auf wirtschaftliche und soziale Herausforderungen in den Ländern der Europäischen Union zu reagieren. Das Unternehmertum kann eine Antwort auf die häufig in bestimmten Bereichen der Gesellschaft konzentrierte hohe Arbeitslosigkeit und niedrige Arbeitsmarktbeteiligung sein, indem es den Menschen ermöglicht, Arbeitsplätze für sich selbst und andere zu schaffen. Somit ist das Unternehmertum von grundlegender Bedeutung für die Entstehung einer stärker unternehmerisch ausgerichteten Wirtschaft, in der neue Unternehmen Lösungen für soziale, wirtschaftliche und ökologische Herausforderungen bieten. Jedoch sind viele Menschen in Bezug auf das Unternehmertum mit bestimmten Hindernissen konfrontiert. Dazu zählen unter anderem der Zugang zu Finanzierung, fehlende unternehmerische Fähigkeiten und ein Mangel an Unternehmernetzwerken. Diese Hürden sind häufig noch ausgeprägter für Menschen aus Gruppen, die innerhalb des Unternehmertums benachteiligt oder unterrepräsentiert sind, da diese vor zusätzlichen Hindernissen stehen, die mit Einstellungen, Motivation und Wohlfahrtssystemen zusammenhängen, die sie in Bezug auf das Unternehmertum diskriminieren. Sowohl politische Maßnahmen im Bereich der Wirtschaftsförderung als auch eine aktive Arbeitsmarktpolitik spielen eine Rolle bei der Überwindung dieser Probleme. Geeignete Maßnahmen können auf nationaler, regionaler und lokaler Ebene ergriffen werden und sich an Schlüsselgruppen wie junge Menschen, Frauen, Ältere, Arbeitslose, Zuwanderer, ethnische Minderheiten und Menschen mit Behinderungen richten. Das vorliegende Kompendium zeigt die Arten von politischen Ansätzen auf, die bei der Überwindung dieser Probleme Wirkung zeigen, und erörtert die Schlüsselfaktoren für ihre erfolgreiche Umsetzung.

Es soll politische Entscheidungsträger innerhalb und außerhalb Europas dazu anregen, neue nationale und lokale Maßnahmen einzuführen, die Menschen aus allen Teilen der Gesellschaft die Möglichkeit einräumen, erfolgreich ein Unternehmen zu gründen und eine selbstständige Erwerbstätigkeit aufzunehmen. Dabei kann auf Erfahrungen zurückgegriffen werden, die sich in der Praxis bereits bewährt haben. Einschlägige Maßnahmen können durch den Einsatz einer Reihe von zu diesem Zweck zur Verfügung stehenden lokalen, regionalen und nationalen Mitteln sowie Mitteln der Europäischen Union, darunter die Europäischen Struktur- und Investitionsfonds sowie nationale Mittel für Maßnahmen der aktiven Arbeitsmarktpolitik, gelenkt und umgesetzt werden. Doch müssen hierfür geeignete Pläne und Vorschläge festgelegt und umgesetzt werden. Dieses Kompendium enthält verschiedenste Beispiele für mögliche Ansätze. Die Beschreibungen dieser politischen Maßnahmen stellen nicht nur die Aktivitäten der einzelnen Programme vor, sondern erörtern auch die Herausforderungen, die sich bei ihrer Entwicklung und Umsetzung ergeben, sowie die Schlüsselfaktoren für eine erfolgreiche Übertragung des jeweiligen Ansatzes auf einen anderen Kontext. Die Absicht dabei ist es, Hilfestellung bei der Übertragung der Erfahrungen und Erkenntnisse zu leisten, damit andere die üblichen Fallstricke vermeiden und schnellere Ergebnisse erreichen können.

Dieses Kompendium ist Teil einer laufenden Zusammenarbeit zum integrationsfördernden Unternehmertum zwischen dem Zentrum für Unternehmertum, KMU und lokale Entwicklung der Organisation für wirtschaftliche Zusammenarbeit und Entwicklung (OECD) und der Generaldirektion Beschäftigung, Soziales und Integration der Europäischen Kommission. Eines der wichtigsten Ziele dieser Zusammenarbeit ist die Unterstützung des Kapazitätsaufbaus für politische Entscheidungsträger durch die Bereitstellung von Nachweisen und Orientierungshilfen zu wirksamen Verfahren. Weitere Ergebnisse sind in Form einer Reihe von Kurzdossiers und Berichten zu den Fehlenden Unternehmern verfügbar, die politische Ratschläge zu speziellen Zielgruppen und politischen Instrumenten bieten. Weitere Informationen zu diesem Arbeitsprogramm sind verfügbar unter: www.oecd.org/employment/leed/inclusive-entrepreneurship.htm. 



\section{Inhaltsverzeichnis}

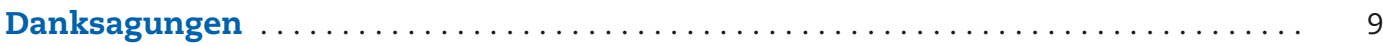

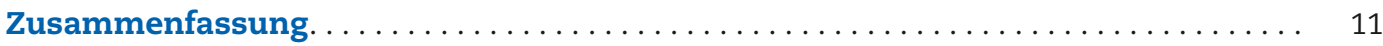

Teil I.

\section{Herausforderungen, Möglichkeiten und Erfolgsfaktoren von Politiken für integrationsfördernde Unternehmensgründungen}

Kapitel 1. Zentrale politische Fragen und Ansätze für integrationsfördernde

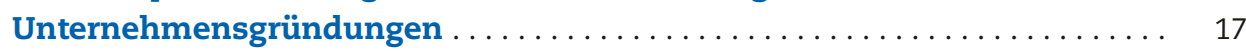

Fakten und Zahlen zu integrationsfördernden Unternehmensgründungen . . . . . . . . 17

Politische Hauptansätze zur Unterstützung des integrationsfördernden

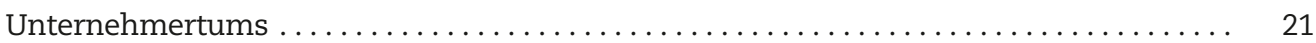

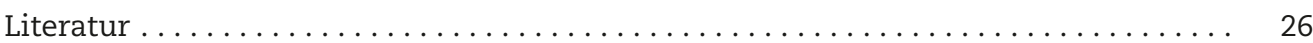

Kapitel 2. Bestandteile erfolgreicher Programme für integrationsfördernde

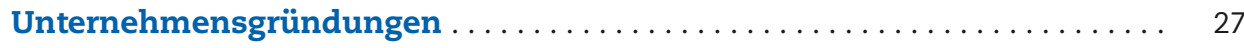

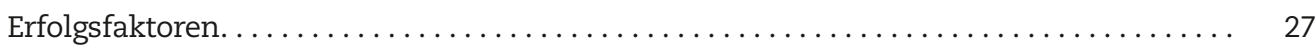

Literatur . . . . . . . . . . . . . . . . . . . . . . . . . . . . . . . . 36

Teil II.

\section{Beispiele für bewährte Verfahren anhand von Programmen für ein integrationsförderndes Unternehmertum}

Unternehmensgründungsprogramm, Österreich $\ldots \ldots \ldots \ldots \ldots \ldots \ldots \ldots \ldots \ldots \ldots \ldots .61$

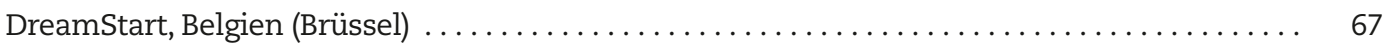

Unternehmertum funktioniert, Belgien (Flandern) $\ldots \ldots \ldots \ldots \ldots \ldots \ldots \ldots \ldots \ldots \ldots \ldots \ldots$

Frauen auf dem Arbeitsmarkt, Kroatien $\ldots \ldots \ldots \ldots \ldots \ldots \ldots \ldots \ldots \ldots \ldots \ldots \ldots \ldots$

Geschäftscoaching für ethnische Minderheiten, Dänemark . . . . . . . . . . . . 86

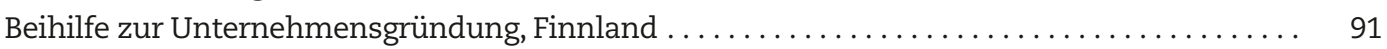

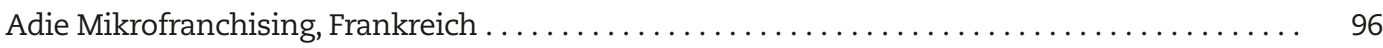

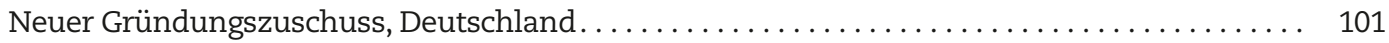

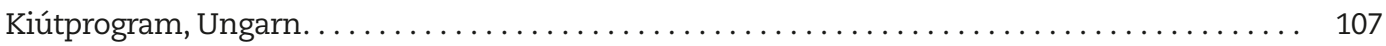

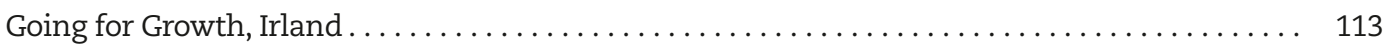

Invitalias Unterstützung für das Unternehmertum Arbeitsloser, Italien . . . . . . . . . . . 120

Massnahme zur Aufnahme einer gewerblichen Tätigkeit oder selbstständigen

Erwerbstätigkeit, Lettland. . . . . . . . . . . . . . . . . . . . . . . . . . . . . . . . . . 127

Förderungsfonds für das Unternehmertum, Litauen . . . . . . . . . . . . . . . . . . . . . . 132

Sozialhilfe für selbstständig Erwerbstätige (BBZ), Niederlande . . . . . . . . . . . . . . . 139

Reifer Unternehmer, Polen . . . . . . . . . . . . . . . . . . . . . . . . . . . 146 


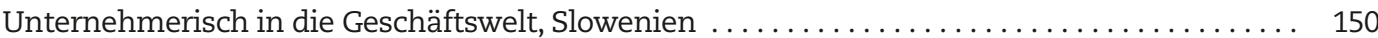

Startrampen für Beschäftigung und Unternehmertum, Spanien . . . . . . . . . . . . . . 156

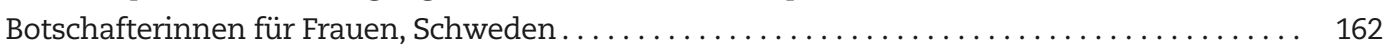

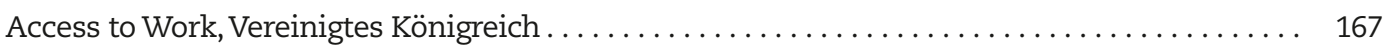

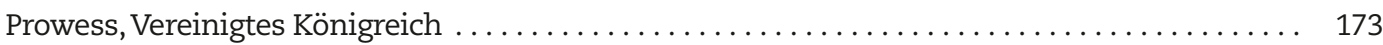

\section{Textfelder}

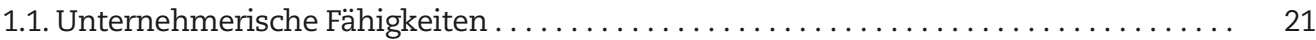

1.2. Kosten aufgrund von Mitnahmeeffekten und Verdrängungseffekte . . . . . . . . . 24

2.1. Beispiele für die Entwicklung und Umsetzung von Projekten im Rahmen von integrierten Strategien . . . . . . . . . . . . . . . . . . . . . . . . . . . . . 29

2.2. Beispiele für die Definition von Zielen $\ldots \ldots \ldots \ldots \ldots \ldots \ldots \ldots \ldots \ldots \ldots \ldots \ldots \ldots .29$

2.3. Ein Beispiel für eine Kontaktaufnahme über die Medien . . . . . . . . . . . . . . 30

2.4. Beispiele für Kontaktaufnahmen durch Mundpropaganda und Veranstaltungen . . . . 30

2.5. Ein Beispiel für die Nutzung von Wettbewerbsmechanismen zur Vergabe von

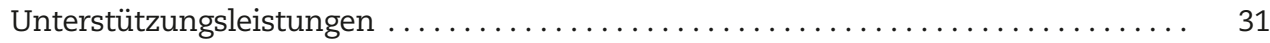

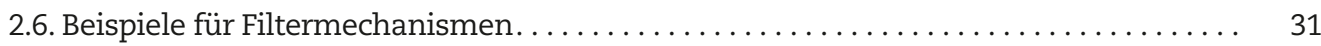

2.7. Beispiele für die Zusammenarbeit mit Partnern bei der Bereitstellung von

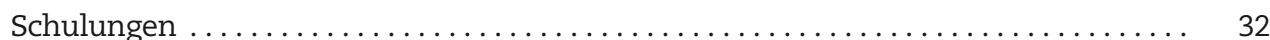

2.8. Ein Beispiel für die Akkreditierung von Organisationen zur Unternehmensförderung. 32

2.9. Beispiele für das Lernen aus Pilotprojekten $\ldots \ldots \ldots \ldots \ldots \ldots \ldots \ldots \ldots \ldots \ldots$

2.10. Beispiele für umfassende Evaluierungen $\ldots \ldots \ldots \ldots \ldots \ldots \ldots \ldots \ldots \ldots \ldots \ldots \ldots . \quad 34$

3.1. Übertragung des österreichischen Unternehmensgründungsprogramms auf

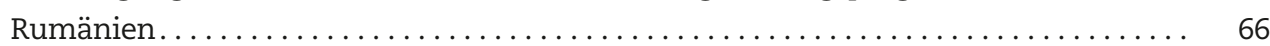

5.1. Blick in die Zukunft: Unternehmertum funktioniert - die Fortsetzung. . . . . . . . . 79

7.1. Profil eines Teilnehmers von „Geschäftscoaching für ethnische Minderheiten“ Maria,

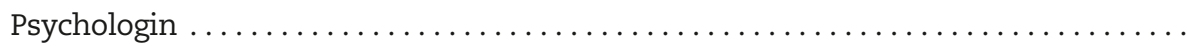

7.2. Profil eines Teilnehmers von „Geschäftscoaching für ethnische Minderheiten“: ein

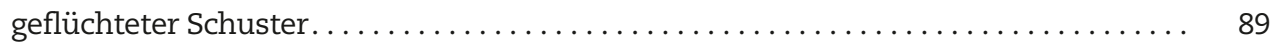

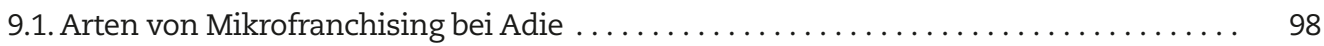

12.1. Die Pilotgesprächsrunde von Going for Growth 2014 in Finnland . . . . . . . . . . . . 118

\section{Tabellen}

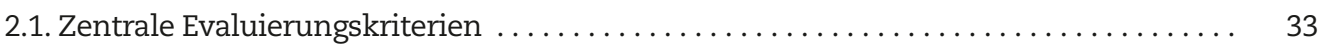

2.2. Übersicht der vorgestellten Politiken und Programme .................. 35

3.1. Überlebensraten für Start-ups im Unternehmensgründungsprogramm . . . . . . . 65

5.1. Profil der Teilnehmer an „Unternehmertum funktioniert“, 2009-2014 . . . . . . . . 75

5.2. Finanzierung von „Unternehmertum funktioniert I, II und III“ $\ldots \ldots \ldots \ldots \ldots \ldots .76$

5.3. Durchschnittliche Kosten pro Beratungsverlauf in „Unternehmertum funktioniert“ . . 76

5.4. Anzahl der Teilnehmer an „Unternehmertum funktioniert“, die in eine selbstständige oder abhängige Erwerbstätigkeit übergingen . . . . . . . 78

13.1. Invitalias Unterstützungsaktivitäten für das Unternehmertum Arbeitsloser . . . . . . . 122

13.2. Ergebnisse von Invitalias Programm zur Unterstützung des Unternehmertums

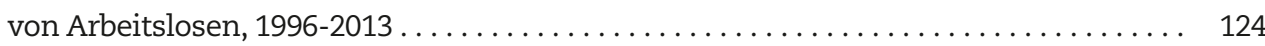

13.3. Persönliche Merkmale der Teilnehmer an Invitalias Programm zur Unterstützung des Unternehmertums von Arbeitslosen . . . . . . . . . . . 125

14.1. Anzahl der Teilnehmer an der Maßnahme zur Aufnahme einer gewerblichen Tätigkeit oder selbstständigen Erwerbstätigkeit 
14.2. Kosten der Maßnahme zur Aufnahme einer gewerblichen Tätigkeit oder selbstständigen Erwerbstätigkeit pro Person aufgeschlüsselt nach Bestandteilen, 2014

15.1. Mittel für den zentralen Fonds des Förderungsfonds für das Unternehmertum, 2009-2015 .

15.2. Auswirkungen des Programms „Förderungsfonds für das Unternehmertum“, 2010-2014

16.1. Finanzierung von BBZ durch die nationale Regierung, 2012 142

16.2. Kosten der einzelnen Wirtschaftsförderungsdienste des BBZ und Klientenanzahl pro Jahr, 2012 143

16.3. Schätzung der Kosten und Nutzen von BBZ pro Existenzgründer . 144

18.1. Schlüsselkennzahlen für die drei Projektphasen von „Unternehmerisch in die Geschäftswelt" 154

19.1. Kosten pro Startrampe. . . . . . . . . . . . . . . . . . . . . . . . . . . 159

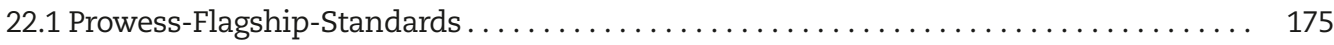

\section{Abbildungen}

1.1. Raten des neuen Unternehmertums in der Europäischen Union, 2009-2013 (kombiniert)

1.2. Vorzug einer selbstständigen Erwerbstätigkeit und deren Realisierbarkeit

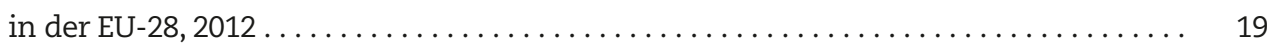

1.3. Hindernisse bei der Unternehmensgründung in der EU-28, $2012 \ldots \ldots \ldots \ldots \ldots \ldots \ldots$

4.1. Die Bestandteile der Schulungen von DreamStart. . . . . . . . . . . . . . . . . 68

9.1. Adie-Mikrofranchising-Rahmen $\ldots \ldots \ldots \ldots \ldots \ldots \ldots \ldots \ldots \ldots \ldots \ldots \ldots \ldots$

13.1. Betriebsprozess, Verwaltung und Aktivitäten von Invitalias Programm zur Unterstützung des Unternehmertums von Arbeitslosen . . . . . . . . 123

15.1. Umsetzung des Förderungsfonds für das Unternehmertum . . . . . . . . . . . . . 134

15.2. Bestandteile des Programms „Förderungsfonds für das Unternehmertum“, 2009-2015. 



\section{Danksagungen}

$\mathrm{D}$ eser Bericht geht auf eine laufende Zusammenarbeit zwischen dem Programm für lokale Wirtschafts- und Beschäftigungsentwicklung (LEED) am Zentrum für Unternehmertum, KMU und lokale Entwicklung der OECD und der Generaldirektion Beschäftigung, Soziales und Integration der Europäischen Kommission zurück.

Der Bericht wurde unter der Leitung von Jonathan Potter, führender Ökonom am Zentrum der OECD für Unternehmertum, KMU und lokale Entwicklung, ausgearbeitet und herausgegeben. David Halabisky, Ökonom im LEED-Programm der OECD, arbeitete an der Ausarbeitung und Redaktion mit und übernahm die Koordination der schriftlichen Beiträge von verschiedenen Mitwirkenden.

Das Material für den Bericht wurde von den folgenden externen Sachverständigen ausgearbeitet: An De Coen, David Deakins, Andrea Dorr, Paula Fitzsimons, Eva Heckl, Jarna Heinonen, Rachida Justo, Alexander Kritikos, Nadine Levratto, Klaas Molenaar, Ricardo Pinto, Peter Ramsden, Petra Reszkető, Arnis Sauka, Karl Wennberg und Patrizia Zanoni.

Der Bericht wurde durch wertvolle Kommentare und Beiträge der Generaldirektion Beschäftigung, Soziales und Integration der Europäischen Kommission ergänzt. Guy Lejeune, Andrea Maier und Risto Raivio leisteten dabei wesentliche Beiträge. Weitere Beiträge stammen von Marco Fantini und Frédéric Mermety, die zur Ausrichtung dieses Kompendiums beigetragen haben.

Und schließlich gilt der Dank der Autoren Eleanor Davies, Barbara Cachova, Bikrim Gill und François Iglesias vom LEED-Programm für ihre Unterstützung. 

INTEGRATIONSFÖRDERNDE UNTERNEHMENSGRÜNDUNG

KOMPENDIUM BEWÄHRTER VERFAHREN

๑) OCDE/Europäische Union, 2016

\section{Zusammenfassung}

F

ast ein Jahrzehnt nach Einsetzen der Wirtschaftskrise im Jahr 2007 ist die Schaffung von Arbeitsplätzen in der gesamten Europäischen Union weiterhin eine politische Priorität. Mit knapp 23 Millionen Arbeitslosen im zweiten Quartal 2015 und weiteren 91 Millionen Nichterwerbstätigen stellt sich allseits die Frage, wie neue Arbeitsplätze geschaffen werden können.

Für das Beschäftigungsproblem existiert keine Patentlösung. Es wird ein mehrgleisiger Ansatz benötigt, der sowohl auf die Stärkung des makroökonomischen Umfelds gerichtete Maßnahmen als auch politische Maßnahmen umfasst, mit denen konkrete Herausforderungen auf dem Arbeitsmarkt angegangen werden. Politiken für ein integrationsförderndes Unternehmertum können ein Bestandteil dieses politischen Maßnahmenpakets sein. Das Ziel dieser Politiken besteht darin, sicherzustellen, dass alle Gruppen von Menschen ungeachtet ihres Hintergrunds und ihrer persönlichen Merkmale eine Chance auf eine Unternehmensgründung oder die Aufnahme einer selbstständigen Erwerbstätigkeit erhalten. Sie legen den Fokus auf die Unterstützung von Menschen im Unternehmertum, die aus sozialen Gruppen stammen, die in Bezug auf das Unternehmertum unterrepräsentiert oder am Arbeitsmarkt benachteiligt sind: junge Menschen, Ältere, Frauen, ethnische Minderheiten und Zuwanderer, Menschen mit Behinderungen und Arbeitslose.

Es ist klar ersichtlich, dass Menschen aus vielen dieser sozialen Gruppen mit geringerer Wahrscheinlichkeit Inhaber eines neuen Unternehmens werden als Männer im zentralen Erwerbsalter. So waren zum Beispiel Frauen in der Europäischen Union zwischen 2009 und 2013 weniger häufig Inhaber eines neuen Unternehmens als Männer (1,8\% gegenüber 3,5\%), obwohl sie über ein ähnliches Niveau an Humankapital verfügten. Im selben Zeitraum waren auch Ältere (50- bis 64-Jährige) weniger häufig Inhaber eines neuen Unternehmens (1,6\% gegenüber 2,6\% bei den Erwachsenen). Junge Menschen (15- bis 30-Jährige) waren hingegen mit derselben Wahrscheinlichkeit wie Erwachsene Inhaber eines neuen Unternehmens (2,9\% bei den jungen Menschen und 2,6\% bei den Erwachsenen), wobei ihre Unternehmen allerdings niedrige Überlebensraten haben. Zu den größten Hindernissen bei der Unternehmensgründung für diese Bevölkerungsgruppen gehören der Zugang zu Finanzierung und fehlende unternehmerische Fähigkeiten. Insbesondere junge Menschen führen diese Hindernisse an. Frauen geben diese Hindernisse genauso häufig an wie Männer. Doch in Bezug auf die Schwierigkeit, die selbstständige Erwerbstätigkeit mit familiären Pflichten zu vereinbaren, und das Fehlen einer Geschäftsidee zeigen sich geschlechtsspezifische Unterschiede. Ältere führen viel seltener Hindernisse für die selbstständige Erwerbstätigkeit an als junge Menschen und Erwachsene im zentralen Erwerbsalter, haben aber dennoch eine relativ niedrige Arbeitsmarktbeteiligung zu beklagen.

Unternehmensgründungen oder, richtiger ausgedrückt, nachhaltige Unternehmensgründungen und damit einhergehend eine größere Arbeitsmarktbeteiligung der anvisierten Bevölkerungsgruppen sind eindeutig ein Schlüsselergebnis, das die Politiken für ein integrationsförderndes Unternehmertum anstreben. Doch zugleich ist die Generierung nachhaltiger Unternehmensgründungen nicht das einzige angestrebte Ergebnis. Menschen, die ermutigt werden, das Unternehmertum in Erwägung zu ziehen und auszuprobieren, werden nicht immer dazu übergehen wollen, ein Unternehmen zu gründen. Sehr häufig gibt ihnen die Beschäftigung mit dem Unternehmertum in Bezug auf ihre Fähigkeiten, ihre Motivation, ihre Netzwerke und ihre Beschäftigungsfähigkeit jedoch Auftrieb, was wiederum ihre Chancen auf einen Arbeitsplatz erhöht. 
Das vorliegende Kompendium bewährter Verfahren soll für das Potenzial der Politiken und Programme für ein integrationsförderndes Unternehmertum sensibilisieren und als Impulsgeber für politische Entscheidungsträger auf nationaler, regionaler und lokaler Ebene dienen. Die ausführlichen Fallstudien aus der gesamten Europäischen Union veranschaulichen einige der zahlreichen Optionen, die politischen Entscheidungsträgern bei der Unterstützung von integrationsfördernden Unternehmensgründungen offenstehen, unter anderem Unternehmerausbildungen, Coaching und Mentoring, Rollenvorbilder, Mikrofinanzierung, Überbrückungshilfen, Unternehmensberatung und unternehmerische Netzwerkarbeit.

Bei der Betrachtung dieser Beispiele lassen sich unabhängig vom gewählten Ansatz und der Klientenzielgruppe mehrere zentrale Erfolgsfaktoren feststellen:

- die Entwicklung und Durchführung von Politiken und Programmen für ein integrationsförderndes Unternehmertum als Teil einer integrierten Strategie;

- die Festlegung klarer Ziele für politische Interventionen;

- der Einsatz von zielgerichteten Kontaktaufnahmen, um benachteiligte und unterrepräsentierte Gruppen einzubinden;

- die Nutzung von Wettbewerbsmechanismen, um Menschen mit Erfolgspotenzial zielgerichtet und intensiv zu unterstützen;

- der Einsatz von qualifizierten Ausbildern und Beratern;

- das Lernen aus Erfahrungen. 


\section{Leseleitfaden}

Dieser Leseleitfaden enthält Informationen zur Nutzung des Kompendiums sowie eine Übersicht seines Aufbaus. Außerdem enthalten sind Informationen über die Erstellung des Kompendiums.

\section{Methodik}

Der erste Schritt im Rahmen der Erstellung dieses Kompendiums war die Entwicklung einer Reihe von Kriterien, anhand derer die aufzunehmenden Programmbeispiele ausgewählt werden. Diese Kriterienliste wurde unter Hinzuziehung verschiedener internationaler Experten im Bereich der Politiken und Programme für ein integrationsförderndes Unternehmertum von der OECD und der Europäischen Kommission entwickelt. Es wurde eine Reihe von Beispielen ausgewählt, um eine relativ breite Abdeckung i) der verschiedenen Regionen der Europäischen Union, ii) der verschiedenen politischen Ansätze, die zur Förderung integrationsfördernder Unternehmensgründungen genutzt werden können, und iii) der verschiedenen sozialen Zielgruppen von Politiken und Programmen für Unternehmensgründungen zu bieten. Als wichtigstes Auswahlkriterium mussten die Beispiele ihre positiven Auswirkungen bei der Schaffung nachhaltiger Unternehmen klar unter Beweis stellen. Außerdem bemühte man sich, Beispiele auszuwählen, die sowohl traditionelle als auch neue, innovative Ansätze herausstellen.

Die Ermittlung von Beispielen erfolgte seitens der OECD durch die Befragung internationaler Experten, politischer Entscheidungsträger und Politiker im Bereich des integrationsfördernden Unternehmertums aus der gesamten Europäischen Union. Aus der daraus resultierenden Liste potenzieller Fälle wurden anhand der Auswahlkriterien die in diesem Buch vorgestellten 20 Beispiele ermittelt. Die in den jeweiligen Beschreibungen enthaltenen Informationen wurden durch Schreibtischstudien und Befragungen der Programmleiter gesammelt. Jede Fallstudie wurde von nationalen Experten und dem Sekretariat der OECD angefertigt.

\section{Nutzung dieses Kompendiums}

Dieses Kompendium besteht aus zwei Teilen. Der erste Teil beginnt mit einem Überblick über wichtige Daten zum integrationsfördernden Unternehmertum in der Europäischen Union in Kapitel 1. Dazu gehören aktuelle Unternehmensgründungszahlen der zentralen Zielgruppen der Politiken für ein integrationsförderndes Unternehmertum (d. h. junge Menschen, Frauen und Ältere) sowie Daten zur Attraktivität und wahrgenommenen Realisierbarkeit einer selbstständigen Erwerbstätigkeit. Kapitel 1 bietet außerdem eine Kurzübersicht der politischen Hauptansätze, die zur Förderung und Unterstützung von Unternehmensgründungen durch benachteiligte und unterrepräsentierte Gruppen genutzt werden.

In Kapitel 2 werden zentrale Erfolgsfaktoren für die wichtigsten politischen Instrumente für ein integrationsförderndes Unternehmertum vorgestellt und erörtert. Das Kapitel enthält zudem eine Übersichtstabelle, in der die jeweiligen Hauptmerkmale der 20 Beispiele vorgestellt werden, sowie eine Liste mit weiterführender Lektüre zum integrationsfördernden Unternehmertum. Zusammenfassungen von je einer Seite für jedes Beispiel der politischen Maßnahmen, die in diesem Buch behandelt werden, finden sich im Anhang zu diesem Kapitel.

InTeil 2 des Buches wird die Sammlung der 20 ausführlichen Beschreibungen der politischen Maßnahmen und Programme vorgestellt. Die Beschreibungen geben die Ziele und Gründe für jedes Beispiel, eine Übersicht der durchgeführten Aktivitäten, die Rolle von Partnem und die erreichten Ergebnisse an. Zudem schildert jede Beschreibung die Herausforderungen, auf die man gestoßen ist, sowie Schlüsselfaktoren für eine erfolgreiche Übertragung des Ansatzes auf einen anderen Kontext. 

TEIL I.

\section{Herausforderungen, Möglichkeiten und Erfolgsfaktoren von Politiken für integrationsfördernde Unternehmensgründungen}



INTEGRATIONSFÖRDERNDE UNTERNEHMENSGRÜNDUNG

KOMPENDIUM BEWÄHRTER VERFAHREN

○ OCDE/Europäische Union, 2016

Kapitel 1.

\title{
Zentrale politische Fragen und Ansätze für integrationsfördernde Unternehmensgründungen
}

\begin{abstract}
In diesem Kapitel werden wichtige Daten zum integrationsfördernden Unternehmertum von jungen Menschen, Frauen und Älteren vorgestellt. Dazu gehören die Raten der Unternehmensgründung, das Interesse am Unternehmertum und Hindernisse für die selbstständige Erwerbstätigkeit. In diesem Kapitel werden außerdem die politischen Hauptansätze erörtert, die in der Europäischen Union zur Förderung und Unterstützung von benachteiligten und unterrepräsentierten Gruppen bei der Unternehmensgründung und selbstständigen Erwerbstätigkeit eingesetzt werden.
\end{abstract}

\section{Fakten und Zahlen zu integrationsfördernden Unternehmensgründungen}

\section{Unternehmensgründungen}

Für politische Entscheidungsträger sind Unternehmensgründungen aus verschiedenen Gründen von Interesse. Neue Unternehmen tragen zur Stimulation von Innovationen bei und sind-ohne Berücksichtigung von Schließungen von und Arbeitsplatzverlusten in größeren etablierten Unternehmen - für die Schaffung von fast allen Arbeitsplätzen im privaten Sektor verantwortlich. Jedoch gründet nur ein relativ geringer Anteil der Bevölkerung ein eigenes Unternehmen, und in Bezug auf diese Neigung bestehen zwischen den Ländern einige wesentliche Unterschiede. Abbildung 1.1 vergleicht die Raten des neuen Unternehmertums für die Gesamtbevölkerung, Frauen, junge Menschen und Ältere. Diese Maßzahlen erfassen den Anteil der Erwachsenen in der Bevölkerung, der angibt, gegenwärtig geschäftsführender Inhaber eines neuen Unternehmens zu sein, das an die Inhaber seit mindestens drei Monaten und höchstens 42 Monaten Gehälter, Löhne oder sonstige Zahlungen geleistet hat. Im Zeitraum zwischen 2009 und 2013 waren rund 2,6\% der Erwachsenenbevölkerung in der Europäischen Union neue Unternehmensinhaber. Auf Ebene der Länder reichte dieser Anteil von 1,5\% in Italien bis 5,0\% in Litauen. Es ist anzunehmen, dass eine Erhöhung der Raten wichtige Vorteile für die Schaffung von Arbeitsplätzen und im Bereich Innovation bietet.

Überdies haben nicht alle Menschen dieselben Möglichkeiten, ein Unternehmen zu gründen und zu führen. Aus Abbildung 1.1 wird deutlich, dass einige Bevölkerungsgruppen mit geringerer Wahrscheinlichkeit Inhaber eines neuen Unternehmens sind. Zwischen 2009 und 2013 waren Frauen weniger häufig Inhaber eines neuen Unternehmens als Männer, obwohl sie über ein ähnliches Niveau an Humankapital verfügten. Innerhalb 
der Europäischen Union waren Frauen in diesem Zeitraum halb so häufig Inhaberinnen neuer Unternehmen wie Männer (1,8\% gegenüber 3,5\%). Diese Lücke lässt sich durch eine Reihe politischer Maßnahmen schließen, unter anderem, indem man Unternehmensgründungen für Frauen attraktiver macht, den Aufbau von Unternehmernetzwerken unterstützt, eine Familienpolitik umsetzt, die Doppelverdiener fördert und Kinderbetreuung anbietet, und die Diskriminierung auf den Finanzmärkten angeht (OECD/EK, 2013).

\section{Abbildung 1.1. Raten des neuen Unternehmertums in der Europäischen Union, 2009-2013 (kombiniert)}

Prozentualer Anteil an der Erwachsenenbevölkerung (15- bis 64-Jährige)

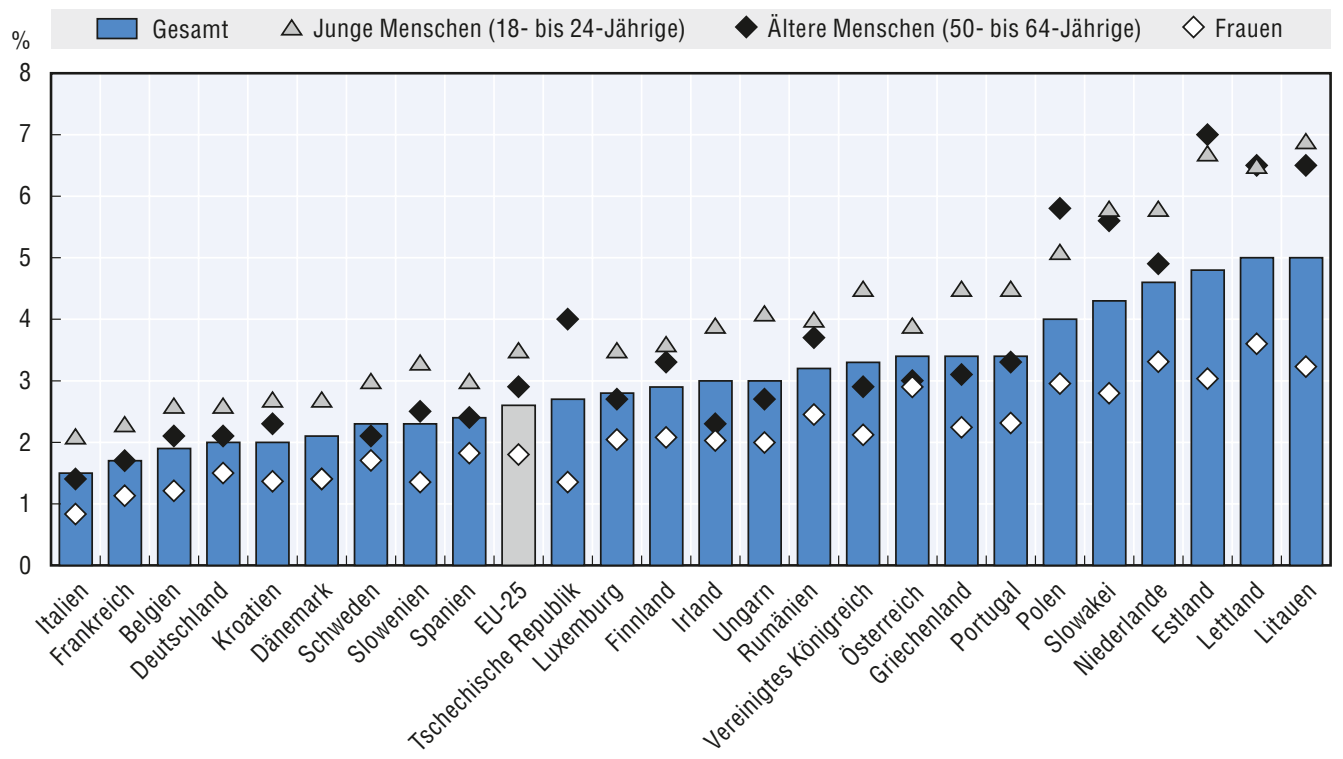

Hinweise: 1. Mitgliedstaaten der Europäischen Union, die im fraglichen Zeitraum an den Erhebungen des Global Entrepreneurship Monitor (GEM) teilnahmen: Belgien, Tschechische Republik, Deutschland, Estland, Irland, Griechenland, Spanien, Frankreich, Kroatien, Italien, Lettland, Litauen, Ungarn, Niederlande, Österreich, Polen, Portugal, Rumänien, Slowenien, Slowakei, Finnland, Schweden, Vereinigtes Königreich. 2. Die in der Abbildung dargestellten Daten wurden über den Zeitraum 2009-2013 aggregiert. Die folgenden Mitgliedstaaten nahmen in diesem Zeitraum nicht an allen jährlichen GEM-Erhebungen teil, wurden aber dennoch einbezogen: Tschechische Republik (2011, 2013), Dänemark (2009, 2010, 2011, 2012), Estland (2012, 2013), Irland (2010, 2011, 2012, 2013), Italien (2009, 2010, 2012, 2013), Litauen (2011, 2012, 2013), Luxemburg (2013), Österreich (2012, 2013), Polen (2011, 2012, 2013), Portugal (2010, 2011, 2012, 2013), Slowakei (2011, 2012, 2013) und Schweden (2010, 2011, 2012, 2013). 3. Die Rate des neuen Unternehmertums ist definiert als Anteil der Erwachsenen, die gegenwärtig geschäftsführender Inhaber eines neu gegründeten Unternehmens sind, wobei ihnen seit mehr als drei, jedoch höchstens 42 Monaten Lohn-, Gehaltsoder sonstige Zahlungen dieses Unternehmens zugehen.

Quelle: Global Entrepreneurship Monitor (GEM) (2014), Besondere Auswertung der jährlichen Erhebungen des Global Entrepreneurship Monitor unter Erwachsenen im Zeitraum 2009-2013.

Junge Menschen (18- bis 24-Jährige) waren hingegen häufiger Inhaber neuer Unternehmen als Erwachsene. Im Zeitraum 2009-2013 waren 3,5\% der jungen Menschen in der Europäischen Union Inhaber neuer Unternehmen. Trotz dieser hohen Gründungsraten deutet eine Vielzahl von wissenschaftlichen Forschungsarbeiten darauf hin, dass von Jungunternehmern geführte Unternehmen häufig niedrige Überlebensraten haben (z. B. van Praag, 2003). Somit besteht die Herausforderung für politische Entscheidungsträger darin, sicherzustellen, dass junge Menschen hochwertige Unternehmen in angemessenen Wirtschaftszweigen gründen, in denen ein ausreichender Nachfrageüberhang besteht, um ein nachhaltiges Unternehmen aufzubauen (OECD/EK, 2012).

Für ältere Unternehmer (50- bis 64-Jährige) ergibt sich ein gemischtes Bild. In einigen Mitgliedstaaten sind ältere Unternehmer mit höherer Wahrscheinlichkeit Inhaber eines neuen Unternehmens, während diese Rate in anderen Mitgliedstaaten unter der Rate der Erwachsenen liegt. Mit fortschreitender Alterung der Bevölkerung der Europäischen Union steigen die Arbeitsmarktbeteiligungsquoten unter den Älteren, 
doch die Selbstständigenquoten bleiben dahinter zurück (OECD/EK, 2012b). Außerdem sind Unternehmen, die von Älteren geführt werden, in der Tendenz klein und haben niedrige Wachstumsraten (OECD/EK, 2012b). Politische Entscheidungsträger können versuchen, das unternehmerische Potenzial von Älteren zu erhöhen, indem sie ein positives Bewusstsein für den Nutzen des Unternehmertums schaffen, den Aufbau von Netzwerken unterstützen, den Zugang zu Startkapital sicherstellen, das Potenzial von Unternehmenserwerben statt von Unternehmensgründungen herausstellen und Ältere dazu ermutigen, Unternehmensgründungen von Jungunternehmern zu unterstützen.

\section{Attraktivität und Realisierbarkeit der selbstständigen Erwerbstätigkeit}

Viele Faktoren beeinflussen die Entscheidung, ein Unternehmen zu gründen. Daten aus der neuesten Eurobarometer-Umfrage deuten darauf hin, dass eine geeignete Geschäftsidee (87\%) und die Fähigkeit, auf die nötige Finanzierung zuzugreifen (84\%), zwei der wichtigsten Faktoren darstellen (EK, 2012). Dieser Entscheidung liegt allerdings die Wahrnehmung des Einzelnen zugrunde, wie attraktiv und realisierbar das Unternehmertum ist. Dabei handelt es sich also um die Frage, ob eine Unternehmensgründung im Vergleich zur Tätigkeit als Angestellter positiv gesehen wird und eine realistische Option darstellt.

Abbildung 1.2 stellt die wahrgenommene Attraktivität und Realisierbarkeit der selbstständigen Erwerbstätigkeit für die Erwachsenenbevölkerung, Frauen, junge Menschen und Ältere dar. Im Jahr 2012 zogen Frauen viel seltener als Männer (nicht abgebildet) eine selbstständige Erwerbstätigkeit der abhängigen Erwerbstätigkeit vor (33\% gegenüber 42\%) und betrachteten die selbstständige Erwerbstätigkeit auch seltener als realisierbar (26\% gegenüber $35 \%$ ). Junge Menschen allerdings betrachteten die selbstständige Erwerbstätigkeit häufiger als Erwachsene als eine der abhängigen Erwerbstätigkeit vorzuziehende (45\% gegenüber 37\%) und realisierbare Tätigkeit (41\% gegenüber 30\%). Diese Begeisterung erklärt die hohen Gründungsraten unter jungen Menschen. Ältere betrachteten die selbstständige Erwerbstätigkeit genauso häufig wie Erwachsene als der abhängigen Erwerbstätigkeit vorzuziehen (35\% gegenüber 37\%), sahen diese jedoch viel seltener als realisierbar an (16\% gegenüber 30\%). Das Gefälle bei der wahrgenommenen Realisierbarkeit ist durch eine Reihe von Faktoren zu erklären. Dazu zählen die Wahl eines bestimmten Lebensstils, finanzielle Negativanreize bei staatlichen Leistungen und der Altersrente sowie zeitliche Opportunitätskosten (d. h., Ältere zeigen häufig weniger Bereitschaft, ihre Zeit Tätigkeiten zu widmen, die künftige Einkommensströme generieren und mit einem gewissen Risiko verbunden sind - etwa die Gründung einer Firma -, gegenüber Tätigkeiten, die sofortige, risikolose Erlöse abwerfen, zum Beispiel eine abhängige Erwerbstätigkeit).

\section{Abbildung 1.2. Vorzug einer selbstständigen Erwerbstätigkeit und deren Realisierbarkeit in der EU-28, 2012}

„Wenn Sie zwischen verschiedenen Arten von Arbeitsplätzen wählen könnten, wären Sie dann lieber selbstständig erwerbstätig?“ „Ungeachtet dessen, ob Sie selbstständig erwerbstätig werden möchten oder nicht, wäre es für Sie realisierbar, innerhalb der nächsten fünf Jahre selbstständig erwerbstätig zu werden?“

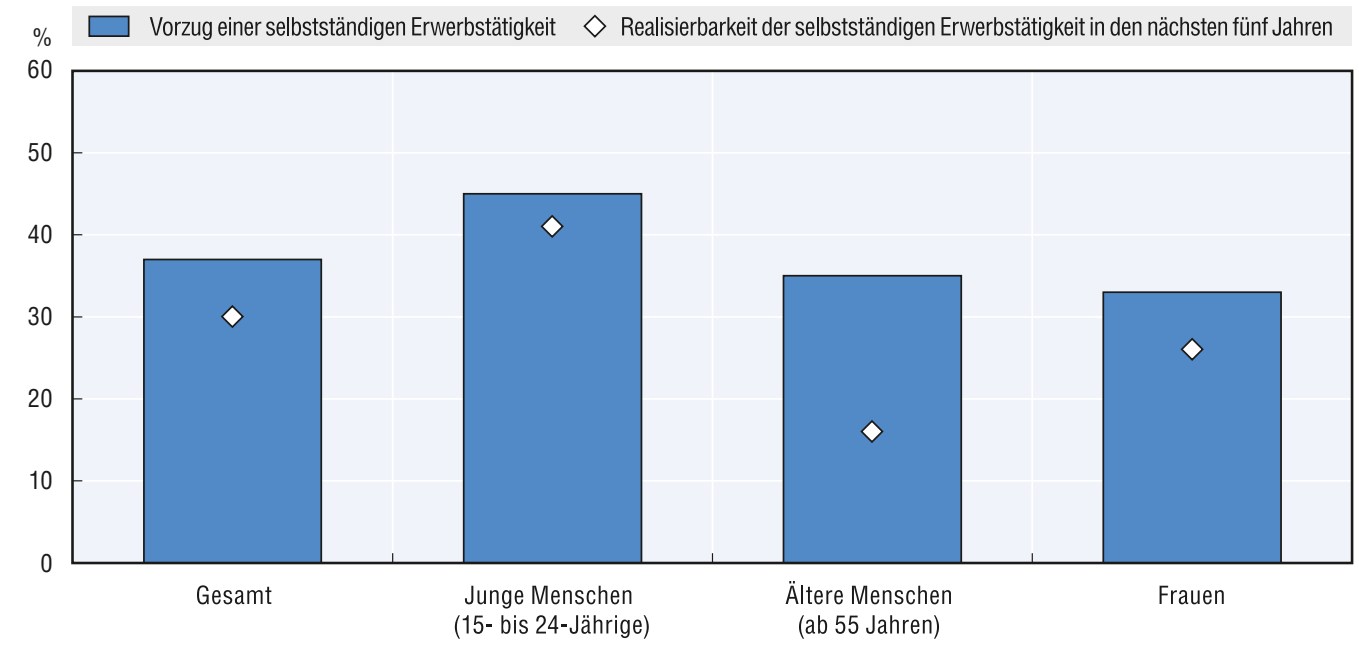

Quelle: Europäische Kommission, 2012, „Entrepreneurship in the EU and beyond”, Flash Eurobarometer 354. 


\section{Hindernisse bei der Unternehmensgründung}

Die wahrgenommenen Hindernisse für die Unternehmensgründung sind in Abbildung 1.3 dargestellt. Der Zugang zu Startkapital ist der am häufigsten angeführte Grund, warum eine selbstständige Erwerbstätigkeit nicht als realisierbar betrachtet wird - dieser Grund wurde von 21\% der Erwachsenen in der Europäischen Union angegeben. Darauf folgte die Angabe fehlender Fähigkeiten für die selbstständige Erwerbstätigkeit (8\%), einer fehlenden Geschäftsidee (7\%) und der Schwierigkeit, die selbstständige Erwerbstätigkeit mit familiären Pflichten zu vereinbaren (6\%). Außerdem angeführt wurden das Risiko eines Scheiterns und seine rechtlichen und sozialen Folgen (5\%) sowie verwaltungstechnische Schwierigkeiten (4\%). Für die öffentliche Politik gibt es Ansatzpunkte zur Verringerung jedes dieser Hindernisse.

\section{Abbildung 1.3. Hindernisse bei der Unternehmensgründung in der EU-28, 2012}

„Warum wäre es für Sie nicht realisierbar, innerhalb der nächsten fünf Jahre selbstständig erwerbstätig zu werden?“

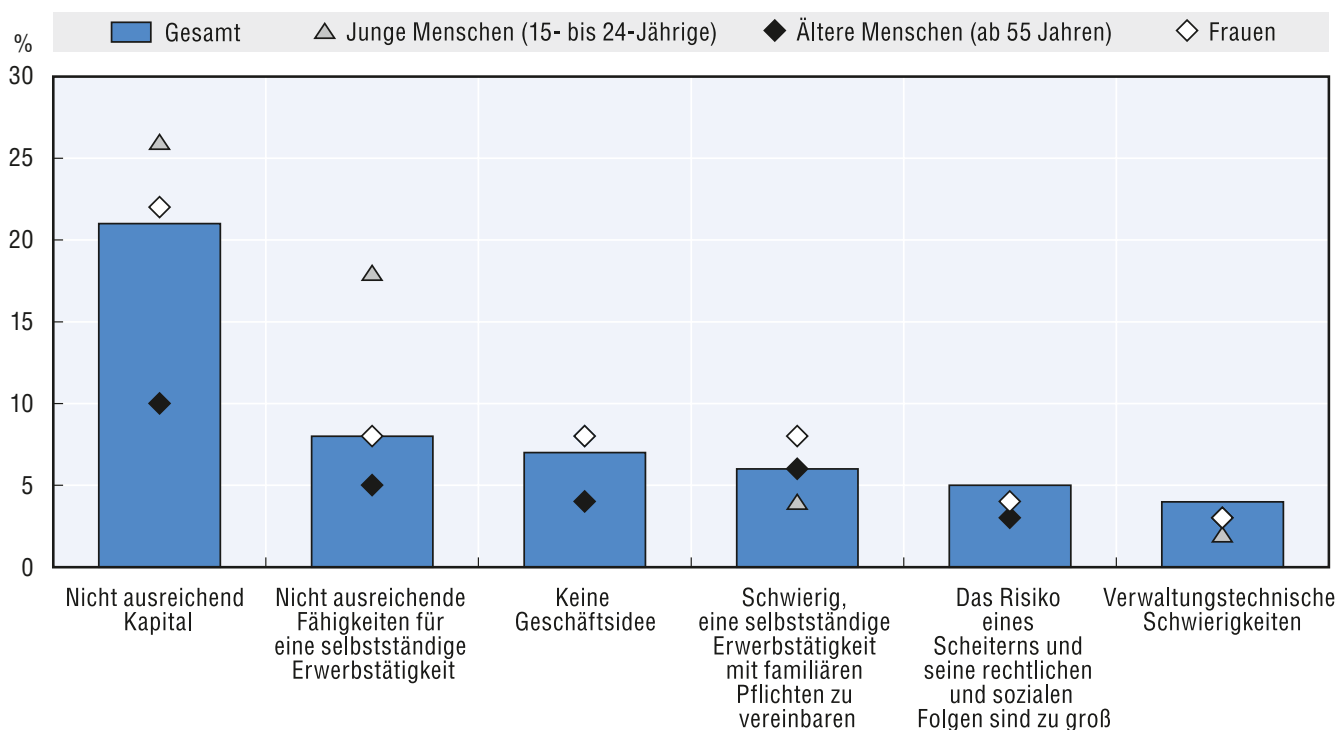

Quelle: Europäische Kommission, 2012, „Entrepreneurship in the EU and beyond”, Flash Eurobarometer 354.

Es bestehen einige Unterschiede im Ausmaß, in dem verschiedene Bevölkerungsgruppen diese Hindernisse zu spüren bekommen. Sowohl Männer als auch Frauen gaben den Zugang zu Kapital für die Unternehmensgründung als das größte Hindernis an, und zwar ähnlich häufig (22\% der Frauen und 20\% der Männer). Auch die Häufigkeit, mit der sie durch einen Mangel an Fähigkeiten, das hohe Risiko eines Scheiterns und die erheblichen Folgen eines Scheiterns des Unternehmens sowie verwaltungstechnische Schwierigkeiten eingeschränkt werden, war bei Männern und Frauen ähnlich hoch. Allerdings gaben Frauen mit höherer Wahrscheinlichkeit die Schwierigkeit, eine selbstständige Erwerbstätigkeit mit ihren familiären Pflichten zu vereinbaren (8\% gegenüber $4 \%$ ), und eine fehlende Geschäftsidee ( $8 \%$ gegenüber 6\%) an.

Junge Menschen stellten die Bevölkerungsgruppe dar, die am häufigsten einen Mangel an Kapital (26\% gegenüber $21 \%$ ) und unternehmerischen Fähigkeiten (18\% gegenüber $8 \%$ ) als Hindernisse bei der selbstständigen Erwerbstätigkeit angaben. In Bezug auf andere wahrgenommene Hindernisse bestanden nur geringe Unterschiede zwischen jungen Menschen und Erwachsenen. Für junge Menschen scheinen verwaltungstechnische Schwierigkeiten kein erhebliches Hindernis zu sein. Dies deutet daraufhin, dass Bemühungen zur Senkung des Verwaltungsaufwands wahrscheinlich nur geringe Auswirkungen auf die unternehmerischen Tätigkeiten junger Menschen haben dürften.

Obwohl Ältere eine selbstständige Erwerbstätigkeit nur halb so häufig als realisierbar ansahen (Abbildung 1.2), gaben sie viel seltener Hindernisse für die selbstständige Erwerbstätigkeit an als junge Menschen oder Erwachsene im zentralen Erwerbsalter. Nur 10\% der Älteren gaben fehlendes Startkapital als Hindernis für die selbstständige Erwerbstätigkeit an. In ähnlicher Weise gaben nur 5\% der Älteren fehlende Fähigkeiten und 4\% fehlende Geschäftsideen als Hindernisse an, während es bei den Erwachsenen $8 \%$ bzw. $7 \%$ waren. 


\section{Politische Hauptansätze zur Unterstützung des integrationsfördernden Unternehmertums}

\section{Unternehmerische Bildung und Ausbildung}

Unternehmerische Bildung an Schulen, Berufsbildung und Hochschulbildung helfen jungen Menschen dabei, eine positive Einstellung gegenüber dem Unternehmertum zu entwickeln und bieten die Möglichkeit, grundlegende unternehmerische Fähigkeiten zu erwerben, damit junge Menschen hochwertigere Unternehmen mit größeren Erfolgschancen gründen können (OECD/EK, 2013).

Die Ziele der unternehmerischen Bildung sind in der Regel je nach Alter der Schüler unterschiedlich. In der Primarstufe besteht das Ziel darin, das Bewusstsein für das Unternehmertum als mögliche berufliche Laufbahn zu schärfen und Wissen, Fähigkeiten und Einstellungen zu entwickeln, die dem unternehmerischen Handeln zuträglich sind. Häufig werden hierzu lokale Unternehmer eingeladen, die im Klassenraum einen Vortrag vor den Schülern über den Betrieb eines Unternehmens halten. Es ist jedoch auch üblich, Schüler in lokale Unternehmen mitzunehmen, damit sie das Alltagsgeschäft eines Kleinunternehmens beobachten und etwas darüber erfahren können.

In der Sekundarstufe wird bei der unternehmerischen Bildung ein größerer Wert auf den Erwerb spezifischer technischer Fähigkeiten gelegt. Den Schülern wird durch Simulationen oder echte Unternehmensgründungen häufig die Möglichkeit gegeben, etwas über Geschäftspläne und den Zugang zu Startkapital zu lernen.

Dieser Ansatz setzt sich in der Hochschulbildung fort. Dort ist es wichtig, dass Studenten die grundlegenden Fähigkeiten für die Gründung und den Betrieb eines Unternehmens erwerben, und lernen, wie wichtig Netzwerke sind. An verschiedenen Universitäten kommen unterschiedliche Ansätze zum Einsatz. Einige bieten in traditionelle Fächer integrierte Module zum Unternehmertum an, während andere spezielle Kurse und Programme anbieten. Viele Hochschuleinrichtungen verfügen zudem über praktisch ausgerichtete Unterstützungsdienste für die Unternehmensgründung, die direkt von der Universität oder über Partnerschaften mit anderen Organisationen in der lokalen Gemeinschaft zur Verfügung gestellt werden.

Berufsbildungszentren sind eng mit der Wirtschaftsgemeinschaft verbunden und bieten somit ein enormes Potenzial zur Unterstützung der unternehmerischen Bildung. Auf dieser Ebene ist die unternehmerische Bildung in der Europäischen Union in der Tendenz weniger entwickelt als auf anderen Bildungsebenen. Aktuelle Ansätze konzentrieren sich häufig auf die Entwicklung von Geschäftsplänen - dies ist zwar ein wichtiger Aspekt, es sollte jedoch stärker darauf geachtet werden, dass die Studenten praktische, in der realen Welt nutzbare Erfahrungen sammeln.

Unternehmerische Fähigkeiten (Textfeld 1.1) können auch außerhalb des Bildungssystems erworben werden. Unternehmerausbildung außerhalb der formellen Bildung lässt sich auf spezifische Gruppen motivierter Unternehmer ausrichten und kann sich eher auf die Entwicklung praktischer Fähigkeiten konzentrieren. Schulungsprogramme bieten in der Regel über mehrere Wochen laufende Module zum Unternehmertum an, allerdings besteht auch erheblicher Raum für die Entwicklung von Online-Kursen. Dies ist einer der politischen Hauptansätze, um den Erwerb unternehmerischer Fähigkeiten für Ältere und benachteiligte junge Menschen zu unterstützen, die nicht am Bildungssystem teilnehmen. Er wird häufig im Rahmen von aktiven Arbeitsmarktmaßnahmen durchgeführt.

\section{Textfeld 1.1. Unternehmerische Fähigkeiten}

Unternehmerische Fähigkeiten sind eine Kombination aus technischen, betriebswirtschaftlichen und persönlichen Fähigkeiten, die für die Gründung und Führung eines Unternehmens und die Aufnahme und Fortführung einer selbstständigen Erwerbstätigkeit erforderlich ist. Hierzu gehören beispielsweise das Erkennen von Möglichkeiten, Fähigkeiten zum Teamaufbau, Verhandlung, Strategieentwicklung, Risikomanagement, Finanzplanung und Marketing. Die Unterstützung des Erwerbs unternehmerischer Fähigkeiten ist nicht nur für die Steigerung der Unternehmensgründungsraten von Bedeutung, sondern auch für die Verbesserung der Qualität von Unternehmensgründungen. 


\section{Coaching und Mentoring}

In der Regel handelt es sich beim Coaching um eine kurze Beziehung, in der die Fähigkeiten eines Unternehmers entwickelt werden sollen. Dabei handelt es sich um einen Prozess der Zusammenarbeit mit klar vorgegebenen Rollen der Teilnehmer. Dabei ist der Betreuer dafür verantwortlich, kurzfristige Ziele zu entwickeln und den Betreuten mittels konstruktiven Feedbacks in Richtung dieses Ziels zu leiten. Der Betreute hingegen soll Ideen und Optionen entwickeln, Schritte zur Erreichung der Ziele ergreifen und Fortschritte melden.

Beim Mentoring handelt es sich auch um eine professionelle Beziehung, bei der eine erfahrene Person (der Mentor) einer anderen Person (dem Mentee) Unterstützung bei der Entwicklung von Fähigkeiten und Wissen bietet, um das berufliche und persönliche Wachstum der weniger erfahrenen Person zu beschleunigen. Diese Beziehungen sind in der Regel längerfristig als beim Coaching angelegt.

Coaching- und Mentoring-Programme können entweder als eigenständige Programme oder als Bestandteil eines integrierten Programms angeboten werden. Damit Coaching- und MentoringBeziehungen ihre Wirksamkeit entfalten, muss zwischen den Beteiligten großes Vertrauen vorherrschen. Für das integrationsfördernde Unternehmertum bedeutet dies, dass ein besonderes Augenmerk auf den Bedürfnissen des Klienten liegen und der Klient einem Betreuer oder Mentor zugewiesen werden muss, der die Herausforderungen versteht, mit denen der Klient konfrontiert ist. Häufig stammt der Betreuer oder Mentor aus derselben Zielgruppe.

Die meisten Initiativen der öffentlichen Politik setzen freiwillige Betreuer und Mentoren aus der lokalen Wirtschaftsgemeinschaft ein. Um die Erfolgschancen zu erhöhen, sollten Initiativen ein Zuordnungsverfahren verwenden, das persönliche wie geschäftliche Eigenschaften berücksichtigt, und die Betreuer und Mentoren eine Schulung zur Stärkung ihrer Kommunikationsfähigkeiten und zur Verbesserung ihrer Kenntnisse über die vor dem Klienten stehenden Herausforderungen durchlaufen lassen (OECD/EK, 2014). Zudem ist es wichtig, die Beziehung zeitlich zu befristen, um die Entstehung einer Abhängigkeitsbeziehung zu vermeiden. Auch sollten die während der Beziehung gemachten Fortschritte verfolgt werden, um sicherzustellen, dass die jeweiligen Erwartungen erfüllt werden.

\section{Finanzierung}

Wie bereits in diesem Kapitel festgestellt, gilt der Zugang zu Startkapital häufig als eines der größten Hindernisse für die Unternehmensgründung. Diese Herausforderung gilt verstärkt für Menschen aus benachteiligten und unterrepräsentierten Gruppen (mit Ausnahme der älteren Unternehmer), was weitgehend den relativen Mangel an Sicherheiten und eigenen finanziellen Mitteln in diesen Gruppen widerspiegelt (OECD/EK, 2013; 2014b). Einige potenzielle Unternehmer sind unter Umständen Diskriminierung auf den Kreditmärkten ausgesetzt. Das Ziel politischer Interventionen für ein integrationsförderndes Unternehmertum sollte in diesem Bereich darin bestehen, dass jeder, ungeachtet des Geschlechts, Alters oder ethnischen Hintergrunds, Zugang zu den am Markt verfügbaren Instrumenten zur Unternehmensfinanzierung erhält.

Beihilfen stellen die am häufigsten verwendeten Ansätze für das integrationsfördernde Unternehmertum dar. Dabei handelt es sich um die Übertragung von Kapital, wobei Gelder von einer Partei (d. h. dem Beihilfegeber) auf eine andere (d. h. den Beihilfeempfänger) übertragen werden. Es ist nicht unüblich, dass öffentliche Programme Beihilfen unter bestimmten Bedingungen vergeben, z. B. in Bezug auf ihre letztendliche Verwendung. Die Festlegung von Bedingungen dient der Verhinderung eines Missbrauchs öffentlicher Ressourcen.

Zwar beantragt nicht jeder Unternehmer einen Bankkredit, doch gibt es zahlreiche Unternehmer, die so vorgehen und deren Antrag abgelehnt wird. Regierungen können hier einschreiten, indem zielgerichtete Kredite an Menschen vergeben werden, die andernfalls nur schwerlich einen Kredit bekommen würden, aber ein tragfähiges Geschäftsprojekt haben (bzw. ein Projekt, das durch ergänzende politische Unterstützung tragfähig werden kann). Viele Länder der Europäischen Union haben subventionierte Darlehensprogramme eingeführt. Ein Beispiel sind sogenannte „Ehrendarlehen“, dank derer viele Generationen von Jungunternehmern und -unternehmerinnen in Italien und Frankreich eine Kombination aus Beihilfen und zinslosen Darlehen erhalten haben. Ehrendarlehen tragen ihre Bezeichnung wegen des 
Umstands, dass sie gegen das Ehrenwort des Darlehensnehmers gewährt werden, ohne dass Sicherheiten oder andere Garantieformen notwendig sind.

Alternativ können politische Entscheidungsträger eine Garantie für privatwirtschaftliche Kredite geben. Bei Kreditgarantieprogrammen handelt es sich um die Verpflichtung einer Agentur, dem Kreditgeber einen Betrag zurückzuerstatten, falls der Kreditnehmer einen Kredit nicht zurückzahlt. Die Gebühr für die Garantie trägt der Kreditnehmer, der Kreditgeber oder beide. Als Mechanismus zur Risikoaufteilung verringern Kreditgarantieprogramme die Risiken und potenziellen Verluste von Gläubigern und veranlassen diese, Kredite an Kreditnehmer mit höherem Risikoprofil zu vergeben. Solche Programme erzeugen in der Regel weniger Marktverzerrungen im Vergleich zur direkten Kreditvergabe, denn sie nutzen bestehende Marktmechanismen (d. h. Privatbanken) als Hauptinstrumente für die Kreditvergabe.

Ein weiterer im Rahmen der Politiken für ein integrationsförderndes Unternehmertum verwendeter Ansatz sind Mikrokredite, also die Gewährung von Kleinstkrediten an Kreditnehmer, denen es häufig an Sicherheiten und einer nachprüfbaren Bonitätsgeschichte mangelt. Diese stammen oft aus benachteiligten Gruppen (z. B. Arbeitslose, junge Menschen oder Menschen mit Behinderungen) und haben unter Umständen keinen Zugang zum regulären Kreditmarkt. Hinter den Mikrokrediten steht nicht nur die Absicht, das Unternehmertum und die selbstständige Erwerbstätigkeit zu unterstützen, sondern auch, die Armut zu lindern. Mikrokredite sind eine Untergruppe der Mikrofinanzierung, bei der eine breitere Auswahl an Finanzdienstleistungen und sonstiger Unterstützung (z. B. Schulungen) für Menschen mit niedrigen Einkommen und keinerlei Ersparnissen bereitgestellt wird. Mikrokredite werden in der Regel über spezialisierte Mikrofinanzierungsinstitutionen zur Verfügung gestellt. In der Europäischen Union liegt die Obergrenze für Mikrokredite bei 25000 EUR. In der Praxis liegen die Mikrokredite, die durch von der Europäischen Union finanzierte Programme (etwa das PROGRESS-Mikrofinanzierungsinstrument oder die EaSI-Finanzierungsinstrumente) unterstützt werden, mit durchschnittlich weniger als 10000 EUR weit unter dieser Obergrenze.

Ein letztes wichtiges Element zur Unterstützung des Zugangs zu Finanzierung im Rahmen der Politiken für ein integrationsförderndes Unternehmertum ist die Finanzbildung. Viele potenzielle Unternehmer aus benachteiligten und unterrepräsentierten Gruppen haben geringe Kenntnisse über grundlegende finanzielle Konzepte und wissen nicht über ihre Möglichkeiten bei der Suche nach Startkapital Bescheid, zum Beispiel wie sie auf neue Formen der Finanzierung wie Crowdfunding und Gruppenkreditvergabe zugreifen können oder wie sie die Verwendung begrenzter Ressourcen durch Bootstrapping maximieren können. Ziel hierbei ist die Verbesserung des Finanzwissens. Dies umfasst das Wissen über und Verständnis von finanziellen Konzepten sowie die Fähigkeiten, die Motivation und das Selbstvertrauen, dieses Wissen und Verständnis anzuwenden, um in verschiedenen finanziellen Kontexten wirksame Entscheidungen zu treffen, das finanzielle Wohlergehen des Einzelnen und der Gesellschaft zu verbessern und eine Beteiligung am Wirtschaftsleben zu ermöglichen.

Programme für ein integrationsförderndes Unternehmertum entwickeln häufig spezifische finanzielle Maßnahmen für benachteiligte und unterrepräsentierte Gruppen als Ganzes oder für Schlüsselgruppen innerhalb dieser Gruppe, zum Beispiel spezielle Initiativen für Frauen oder junge Menschen. Allerdings entscheiden sich kleinere Länder und Regionen häufig, diese Gruppen über allgemeine, der gesamten Bevölkerung zur Verfügung stehende Programme zu bedienen, wenn die Größe der Klientel kein maßgeschneidertes Programm rechtfertigt. Bei der Einrichtung von Finanzprogrammen ist unbedingt anzuerkennen, dass die Verfügbarkeit einer Finanzierung allein kaum alle bei der Unternehmensgründung auftretenden Probleme lösen kann. Finanzierungsprogramme müssen auch auf andere Initiativen abgestimmt sein, einschließlich derer zur Förderung von unternehmerischen Fähigkeiten und Marktentwicklungsfähigkeiten.

\section{Überbrückungshilfen}

Programme zur Überbrückungshilfe zahlen für einen begrenzten Zeitraum nach der Unternehmensgründung eine Zuwendung oder Arbeitslosenunterstützung, um die Sozialversicherungsbeiträge und persönlichen Unterhaltskosten zu decken. Dieser Ansatz wurde im letzten Jahrzehnt von mehreren Regierungen in der Europäischen Union eingeführt, um sicherzustellen, dass sich Arbeitslose während der Phase der Unternehmensgründung und -entwicklung bzw. 
der Aufnahme einer selbstständigen Erwerbstätigkeit ein Einkommen sichern können, bevor sie regelmäßige Einnahmen erwirtschaften.

Dank der großen Anzahl von Überbrückungshilfeprogrammen, die in der Europäischen Union umgesetzt werden, können umfassende Belege Aufschluss über die Wirksamkeit dieser Programme geben. Die Überlebensraten nach zwei Jahren liegen in der Regel zwischen 66\% und 85\% - diese Zahlen liegen nur knapp unter der Überlebensrate neuer Unternehmen insgesamt (OECD/EK, 2014). Allerdings reicht eine Untersuchung der Überlebensraten nicht zur Prüfung der Wirksamkeit und Effizienz dieser Programme aus. Auch die Programmkosten sowie die Kosten aufgrund von Mitnahme- und Verdrängungseffekten sind zu berücksichtigen. Die Schätzungen der Kosten aufgrund von Mitnahmeeffekten (üblicherweise selbst bekundete Angaben zu „Ich hätte unabhängig von den Zuschüssen ein Unternehmen gegründet“) unterscheiden sich je nach Land und Merkmalen des Programms. Verdrängungseffekte werden in Evaluierungen selten untersucht, sollten aber von politischen Entscheidungsträgern berücksichtigt werden.

\section{Textfeld 1.2. Kosten aufgrund von Mitnahmeeffekten und Verdrängungseffekte}

Kosten aufgrund von Mitnahmeeffekten: Kosten für die Unterstützung von Teilnehmern, die auch ohne Zuschüsse ein neues Unternehmen gegründet hätten. Da das Verhalten dieser „unproduktiven Teilnehmer" nicht durch das Programm beeinflusst wird, trägt ihreTeilnahme nicht zum wirtschaftlichen Wert bei, sondern verursacht öffentliche Ausgaben. Die sozialen Kosten dieser Ausgaben sind die Summe der wettbewerbsverzerrenden Kosten, der übermäßigen Steuerlast, über die dieses Programm finanziert wird, und die Kosten zur Deckung der Einrichtungs- und Betriebskosten.

Verdrängungseffekte: Der Umfang, in dem bezuschusste Unternehmen nicht bezuschusste Unternehmen um Märkte bringen und diese verdrängen.

\section{Unterstützung für die Unternehmensentwicklung}

Bei Unterstützungsleistungen für die Unternehmensentwicklung handelt es sich um Dienstleistungen, mit denen die Ergebnisse des Unternehmens durch die Verbesserung seiner Wettbewerbsfähigkeit und seines Marktzugangs verbessert werden sollen. Zu den Unterstützungsleistungen gehören die Bereitstellung von Informationen, Beratungsdienstleistungen, Marketingunterstützung, Unterstützung bei Technologieentwicklung und -transfer und Netzwerkarbeit. Dazu zählt die Unterstützung sowohl für strategische (mittel- bis langfristige Themen zur Verbesserung der Leistung) als auch betriebliche (alltägliche) Themen (siehe OECD/EK, 2014).

In der Praxis setzen politische Entscheidungsträger vielfältige Instrumente als Teil ihrer Unterstützungspakete für die Unternehmensentwicklung ein. Die Dienstleistung mit der geringsten Intensität ist die Orientierungshilfe. Hierbei werden Unternehmern Informationen darüber bereitgestellt, wo sie professionelle Informations- und Hilfequellen finden können. Dies kann über Websites, Informationen, die über die öffentlichen Arbeitsverwaltungen oder andere Partner (z. B. Handelskammern) zur Verfügung gestellt werden, oder Medienkampagnen erfolgen. Der kritischste Erfolgsfaktor bei der Bereitstellung von Informationen zur Orientierungshilfe für Unternehmer aus benachteiligten und unterrepräsentierten Gruppen liegt in der Nutzung bereits verwendeter Kanäle (z. B. Gemeinschaftsmedien).

Die Unternehmensberatung ist ein Wirtschaftsförderungsdienst, der dem Unternehmer professionelle Unternehmensberatung bietet. Eine gängige Herangehensweise besteht darin, die Unternehmensberatung als Teil eines integrierten Unterstützungspakets bereitzustellen. Regelmäßige Treffen mit Unternehmensberatern sind häufig Voraussetzung für eine finanzielle Unterstützung.

Die intensivsten Unterstützungsleistungen für die Unternehmensentwicklung werden erbracht, wenn Unternehmen in eigens dafür vorgesehenen Orten untergebracht sind. Bei Gründungszentren handelt es sich um Einrichtungen zur Förderung der Gründung und des Wachstums von Unternehmen mithilfe einer Reihe von Ressourcen und Dienstleistungen zur Unternehmensförderung, die sowohl direkt im Gründungszentrum als auch über dessen Kontaktnetz angeboten werden. Gründungszentren weisen Unterschiede bei der Art der Bereitstellung ihrer Dienstleistungen, ihrer Organisationsstruktur und ihren 
Klientengruppen auf. Es gibt zwar virtuelle/online erreichbare Gründungszentren, die meisten Programme nehmen die Unternehmen in der Phase vor und während ihrer Gründung jedoch für einen begrenzten Zeitrauminihren Räumlichkeiten auf.DererfolgreicheAbschlusseinesUnternehmensgründungsprogramms steigert die Wahrscheinlichkeit für das Überleben und Wachstum des neu gegründeten Unternehmens.

\section{Netzwerkarbeit}

Unternehmernetzwerke bieten Zugang zu Gruppen untereinander verbundener Unternehmer, Unternehmensdienstleistern und verschiedenen sonstigen relevanten Menschen, über die Unternehmer im Rahmen von auf Gegenseitigkeit basierenden Beziehungen Zugang zu Informationen und Ideen für den Betrieb ihrer Unternehmen erhalten. Diese Netzwerke können Unternehmern dabei helfen, sich eine Finanzierung zu sichern, Geschäftspartner, Lieferanten, Angestellte und Kunden zu finden, und auf Ideen für neue Produkte, Verfahren, Organisationsmethoden und Geschäftsmodelle zu kommen. Außerdem können sie die Wahrnehmung des Einzelnen im Hinblick auf die Attraktivität und Realisierbarkeit des Unternehmertums beeinflussen (OECD/EK, 2015).

Für den Erfolg dieser Initiativen sind mehrere zentrale Faktoren maßgeblich. Erstens sollten die Netzwerkstrukturen und -verfahren so gestaltet sein, dass ein hohes Maß an Interaktion zwischen den Unternehmern im Netzwerk und dem weiteren Wirtschaftsumfeld gewährleistet ist. Damit wird sichergestellt, dass Unternehmer aus benachteiligten und unterrepräsentierten Gruppen Zugang zu einem größeren Ressourcen-Pool haben, um ihnen bei der Überwindung von Hindernissen und Hürden zu helfen. Zudem sorgt eine starke Brücke zwischen dem Netzwerk und dem weiteren Wirtschaftsumfeld dafür, dass das Netzwerk die Gruppe benachteiligter Unternehmer nicht noch weiter isoliert und ihre Benachteiligung verstärkt.

Zweitens sollten politische Entscheidungsträger klar definierte Ziele für Unternehmernetzwerke festlegen, die sich prägend auf die Netzwerkstruktur und -verfahren auswirken. So kombinieren einige politische Maßnahmen zum Beispiel den Aufbau von Netzwerken mit der Bereitstellung von Unternehmerschulungen und Zugang zu Finanzierung. Für solch ein Netzwerk ist eine vollkommen andere Struktur erforderlich als für ein Netzwerk, dessen Ziel im Aufbau internationaler Verbindungen und in der Förderung von Export und Geschäftsausweitung besteht. Ein klar definierter Zweck sorgt zudem für eine verbesserte Kontaktaufnahme und Sensibilisierung im Netzwerk.

Drittens sollten politische Entscheidungsträger kein Überangebot an Netzwerken schaffen. Existiert eine große Zahl von Netzwerken, verdrängen diese sich gegenseitig und untergraben die Vorteile, die jedes einzelne von ihnen mit sich bringt. Viel wichtiger ist es, dass sich politische Entscheidungsträger auf die Bereitstellung hochwertiger Netzwerke konzentrieren.

Viertens sollte die öffentliche Politik die Nutzung von Online-Unternehmernetzwerken fördern. Über deren Wirksamkeit ist zwar nichts bekannt, doch sind sie kostengünstig und einfach zugänglich. Allerdings müssen politische Entscheidungsträger sicherstellen, dass das Netzwerk dynamisch ist, damit die Mitglieder sich kontinuierlich einbringen. Die Auswirkungen sind wahrscheinlich größer, wenn die Online-Netzwerke mit persönlichen Interaktionen kombiniert werden.

Und schließlich istes von zentraler Bedeutung, dass politische Entscheidungsträger Netzwerkinitiativen so gestalten, dass die Verwaltung des Netzwerks und das Eigentum am Netzwerk auf seine Mitglieder übergehen. Dies sorgt für mehr Vertrauen unter den Mitgliedern und potenziellen Mitgliedern und für eine stärke Beteiligung. 


\section{Literatur}

Europäische Kommission, 2012, „Entrepreneurship in the EU and beyond“, Flash Eurobarometer 354.

Global Entrepreneurship Monitor (GEM) (2014), Besondere Auswertung der jährlichen Erhebungen des Global Entrepreneurship Monitor unter Erwachsenen im Zeitraum 2009-2013.

OECD/Europäische Kommission (2015), „Kurzdossier zu Netzwerken für ein integrationsförderndes Unternehmertum“, OECD Publishing, Paris, und EU Publishing, Luxemburg. ec.europa.eu/social/ BlobServlet? docId=13739\&langId=de

OECD/Europäische Kommission (2014), Die fehlenden Unternehmer 2014: Politiken für ein integrationsförderndes Unternehmertum in Europa, OECD Publishing, Paris, und EU Publishing, Luxemburg. http://dx.doi.org/10.1787/9789264230897-de

OECD/Europäische Kommission (2014b), „Zugang zu Gründungsfinanzierung für ein integrationsförderndes Unternehmertum“, OECD Publishing, Paris, und EU Publishing, Luxemburg. ec.europa.eu/social/BlobServlet?docId=11499\&langId=de

OECD/Europäische Kommission (2013), Die fehlenden Unternehmer: Politiken für ein integrationsförderndes Unternehmertum in Europa, OECD Publishing, Paris, und EU Publishing, Luxemburg. http://dx.doi.org/10.1787/9789264188419-de

OECD/Europäische Kommission (2012), „Kurzdossier zur unternehmerischen Initiative junger Menschen“, OECD Publishing, Paris, und EU Publishing, Luxemburg. http://ec.europa.eu/social/ BlobServlet?docId=8759\&langId=de

OECD/Europäische Kommission (2012b), „Kurzdossier zur unternehmerischen Initiative älterer Menschen“, OECD Publishing, Paris, und EU Publishing, Luxemburg. ec.europa.eu/social/ BlobServlet? docId=9644\&langId=de

van Praag, M. (2003), „Business Survival and Success of Young Small Business Owners: An Empirical Analysis“, Small Business Economics, Bd. 21, S. 1-17. 


\title{
Bestandteile erfolgreicher Programme für integrationsfördernde Unternehmensgründungen
}

\begin{abstract}
In diesem Kapitel werden sechs zentrale Erfolgsfaktoren für Politiken und Programme für ein integrationsförderndes Unternehmertum vorgestellt und erörtert. In der Diskussion werden Verbindungen zwischen den Erfolgsfaktoren und den in diesem Kompendium enthaltenen Beispielen gezogen. Das Kapitel stellt zudem jedes Beispiel für eine politische Maßnahme in einer zusammenfassenden Tabelle von je einer Seite und einer Übersichtstabelle mit den jeweiligen Hauptmerkmalen vor. Den Abschluss bildet eine Liste mit weiterführender Lektüre.
\end{abstract}

\section{Erfolgsfaktoren}

Programme für Unternehmensgründungen und selbstständige Erwerbstätigkeit unter benachteiligten und unterrepräsentierten Gruppen müssen der Art und dem Umfang der Hindernisse, die diesen Gruppen in Bezug auf eine erfolgreiche Unternehmensgründung im Wege stehen, im Unterschied zur allgemeinen Bevölkerung und anderen benachteiligten und unterrepräsentierten Gruppen Rechnung tragen.

Die Programme müssen außerdem auf vielfältige Probleme reagieren, auf die bestimmte Zielgruppen beim Zugang zu Programmen stoßen. Frauen zum Beispiel bevorzugen eher einen lokalen Zugang zu Dienstleistungen zur Unternehmensförderung wie Schulungen und Mentoring, junge Menschen haben eher mit der Skepsis seitens allgemeiner Unternehmensberater zu kämpfen, ethnische Minderheiten und Zuwanderer wissen häufig nicht, welche Unterstützungsleistungen ihnen zur Verfügung stehen, und Menschen mit Behinderungen benötigen eine Rückversicherung bezüglich ihres Sozialversicherungsstatus (OECD/EK, 2013).

Um auf diese beiden Bedürfnisse einzugehen, können politische Entscheidungsträger bei der politischen Umsetzung verschiedene Modelle nutzen - von der vollständigen Integration in die Erbringung allgemeiner Dienste bis hin zum Einsatz von spezialisierten Agenturen, die maßgeschneiderte Unterstützungsleistungen für bestimmte Zielgruppen bereitstellen. Die Wahl des Modells hat Auswirkungen auf die Wirksamkeit und die Kosten der politischen Intervention.

Daher stellt sich für politische Entscheidungsträger die zentrale Frage, inwiefern benachteiligte und unterrepräsentierte Gruppen in angemessener Weise durch dieselben Unterstützungs- und Bereitstellungsvorkehrungen bedient werden können, die auch anderen Bevölkerungsgruppen angeboten 
werden (d. h. allgemeine Unterstützungsleistungen), und inwiefern es sinnvoll ist, teilweise oder vollständig davon abweichende politische Maßnahmen und Bereitstellungswege (d. h. zielgerichtete Unterstützung) anzubieten. Zielgerichtete Programme und Bereitstellungsstrukturen bieten den Vorteil, dass sie dazu beitragen, dass sichergestellt wird, dass die am schwersten erreichbaren Gruppen Unterstützung erhalten, und zwar die Art von Unterstützung, die am ehesten für sie geeignet ist. Andererseits kann eine zu starke Orientierung in Richtung unterschiedlicher Unterstützungsangebote und -strukturen aufgrund der hohen Kosten und fehlenden Skaleneffekte bei der Bereitstellung sowie der Isolation von Zielgruppen gegenüber anderen relevanten Programmen problematisch sein.

Unabhängig vom verwendeten Bereitstellungsmodell sind sechs zentrale Erfolgsfaktoren für die Gestaltung und Bereitstellung von Politiken und Programmen für ein integrationsförderndes Unternehmertum zu nennen.

\section{Entwicklung und Durchführung von Politiken und Programmen für ein integrationsförderndes Unternehmertum als Teil einer integrierten Strategie}

Auch wenn in der Europäischen Union immer häufiger Politiken für ein integrationsförderndes Unternehmertum zum Einsatz kommen, sind die resultierenden politischen Maßnahmen in vielen Mitgliedstaaten der Europäischen Union über verschiedene Regierungsministerien und Regierungsebenen sowie über viele allgemeine und spezialisierte Organisationen zur Unternehmensförderung verteilt. Um sicherzustellen, dass es bei diesen politischen Maßnahmen keine großen Lücken und keine Doppelarbeit gibt, dass die Maßnahmen sich gegenseitig verstärken, dass Engpässe erkannt und beseitigt werden, dass erfolgreiche politische Innovationen verbreitet werden und dass Unternehmer und potenzielle Unternehmer die für sie geeigneten Unterstützungsleistungen finden können, sollten politische Maßnahmen gemeinsam als Teil einer Strategie für das integrationsfördernde Unternehmertum gestaltet werden, die mit der Unterstützung für das allgemeine Unternehmertum verknüpft ist. Die Entwicklung dieser Strategien sollten auf einem Verständnis des politischen Kontexts, der Ermittlung der zentralen Akteure und der bereits vorhandenen politischen Unterstützungsleistungen, der Ermittlung der erforderlichen Maßnahmen und der Nutzung von Überwachungs- und Evaluierungsverfahren basieren, um die politischen Eingriffe zu verbessern.

Zur Unterstützung der politischen Entscheidungsträger bei der Gestaltung einer Strategie stehen eine Reihe von Schritten und Instrumenten zur Verfügung. Eines davon ist das Konzept des politischen Zyklus, das den politischen Entwicklungsprozess und den jeweiligen Informationsbedarf in verschiedenen Programmphasen hervorhebt (siehe OECD/EK, 2013). Eher praktisch ausgerichtet ist das diagnostische Instrument „COPIE“ (www.cop-ie.eu/). Es hilft politischen Entscheidungsträgern in bestimmten Gebieten bei der Sammlung quantitativer und qualitativer Informationen zur aktuellen politischen Umgebung und bei der Ermittlung von Bereichen mit Verbesserungspotenzial. Außerdem bedarf es eines Verfahrens zur Maßnahmenplanung, im Rahmen dessen Interessenträger diese diagnostischen Informationen in Ziele und priorisierte Maßnahmen übersetzen, sowie eines Verfahrens zur Auswahl finanzierungswürdiger Projekte innerhalb eines Programms und zur Bestimmung derjenigen politischen „Bottom-up“-Innovationen, die fortlaufende oder erweiterte Unterstützung verdienen. Die Programmevaluierung schließlich ist ein Instrument, das in der Europäischen Union zu wenig genutzt wird. Sie ist von wesentlicher Bedeutung, weil sie den politischen Entscheidungsträgern dabei hilft, die weiteren Auswirkungen politischer Maßnahmen (neben Mitnahme- und Verdrängungseffekten) zu verstehen. Eine gute Strategie hängt nicht nur von der Sammlung solcher Evaluierungsnachweise, sondern auch von deren Nutzung in allen Phasen des politischen Zyklus ab. 


\section{Textfeld 2.1. Beispiele für die Entwicklung und Umsetzung von Projekten im Rahmen von integrierten Strategien}

Dieses Kompendium enthält mehrere Beispiele für Programme und Projekte, die als Teil breiter angelegter Unterstützungsprogramme gestaltet und umgesetzt wurden. Access to Work im Vereinigten Königreich ist ein Beihilfeprogramm, das Arbeitsplatzanpassungen (z. B. physische Veränderungen, Anschaffung von Ausrüstung) für Menschen mit Behinderungen unterstützt. Selbstständig Erwerbstätige mit Behinderungen haben Zugang zu allen Beihilfen in diesem Programm und Bewerber, die sich für eine Unternehmensgründung interessieren, werden von den Programmmitarbeitern an das Programm „New Enterprise Allowance“ („Zuwendung für neue Unternehmen“) weitergeleitet. Access to Work wurde im Rahmen breiter angelegter Sozialprogramme für Menschen mit Behinderungen entwickelt. Die Inanspruchnahme dieses Programms verringert keine anderen bezogenen Unterstützungsleistungen.

Botschafterinnen für Frauen in Schweden wurde als Teil einer nationalen Strategie zur Werbung für und Unterstützung des weiblichen Unternehmertums entwickelt. Das Botschafterinnen-Programm startete 2008 mit der Absicht, bei Frauen für das Unternehmertum zu werben. Weitere Programme wurden aufgesetzt, um das Programm durch die Bereitstellung von Schulungen, Finanzierung und Unternehmensberatung zu ergänzen.

Frauen auf dem Arbeitsmarkt war Teil einer Reihe von Heranführungsprogrammen in Kroatien, die das Angebot der öffentlichen Arbeitsverwaltung verbessern sollten. Dieses Programm leistete Beamten technische Unterstützung und bot eine Reihe von Beschäftigungsprogrammen an, unter anderem drei Projekte zur Unterstützung von Unternehmerinnen.

\section{Definition klarer Ziele der politischen Intervention}

Die Definition geeigneter Ziele ist einer der wichtigsten Schritte bei der Politikgestaltung, denn aus diesen Zielen ergeben sich die zu ergreifenden Maßnahmen, das passende Bereitstellungsmodell und die zur Messung des Programmerfolgs anzuwendenden Kriterien. Zur Definition der Ziele müssen politische Entscheidungsträger eine Bedarfsdiagnose und eine Maßnahmenplanung vornehmen. Diese Tätigkeiten beinhalten die Zusammenstellung von Informationen und Nachweisen zu den Problemen, mit denen benachteiligte und unterrepräsentierte Gruppen bei der Unternehmensgründung und der Aufnahme einer selbstständigen Erwerbstätigkeit konfrontiert sind, zu den aktuellen Angeboten der Politik sowie zu den Möglichkeiten zur Verbesserung politischer Maßnahmen und zur Beseitigung von Lücken in diesen Maßnahmen. Diese Informationen und Nachweise werden für Vorschläge zu den am besten geeigneten Maßnahmen herangezogen, die künftig umzusetzen sind. Ziele sollten festgelegt werden, die bei der Entwicklung und Bereitstellung der Programme als Orientierung dienen und dafür sorgen, dass die Programme einen angemessenen Umfang haben.

Auch Ex-ante-Evaluierungen sind sehr wichtig. Sie werden vor einer politischen Intervention durchgeführt, um deren Relevanz und Kohärenz sowie die für ihre Umsetzung getroffenen Vorkehrungen zu bewerten. Die Ex-ante-Evaluierung kann genutzt werden, um Ziele und Meilensteine für Aktivitäten, Leistungen und Ergebnisse zu bestimmen und Verfahren für spätere Evaluierungen festzulegen, die während der Laufzeit der Maßnahme vorzunehmen sind.

\section{Textfeld 2.2. Beispiele für die Definition von Zielen}

Dieses Kompendium enthält viele Beispiele für Verfahren, bei denen zur Definition von Zielen eine Bedarfsanalyse eingesetzt wurde. Kiútprogram in Ungarn führte ein Gruppenkreditvergabeprogramm für Roma in benachteiligten Regionen ein, um Unternehmensgründungen und die Formalisierung von informellen Unternehmen zu unterstützen. Als erster Schritt bei der Entwicklung dieses Programms wurde in potenziellen Regionen Feldforschung betrieben, um den Bedarf an einem solchen Programm einzuschätzen.

Das Mikrofranchising-Programm von Adie in Frankreich wurde vor dem Hintergrund einer wachsenden Zahl von Klienten entwickelt, die bereit waren, ein Unternehmen zu gründen,jedoch keine tragähige Geschäftsidee hatten. Traditionelle Start-ups und Franchising sind für Unternehmer mit niedrigem Einkommen nicht erschwinglich - das Mikrofranchising-Modell bietet eine Lösung für dieses Problem. Das Programm rechnet auch mit einem Wachstum und legt dafür 5-Jahres-Ziele für jede Art des Mikrofranchising-Modells fest. 


\section{Einsatz von zielgerichteten Kontaktaufnahmen, um benachteiligte und unterrepräsentierte Gruppen einzubinden}

Die Fähigkeit, potenzielle Klienten zu erreichen, liegt dem Erfolg jedes Programms oder Projekts für ein integrationsförderndes Unternehmertum zugrunde. Öffentliche Agenturen bzw. die Einrichtungen, die Unterstützungsleistungen bereitstellen, sollten sich um die Ausschöpfung der Medienkanäle bemühen, die von den Mitgliedern der verschiedenen anvisierten Gruppen am häufigsten genutzt werden. Dazu gehören zum Beispiel Radio, Zeitungen und Social Media, die bereits eine starke Anhängerschaft in der Klientenzielgruppe haben. Zusätzlich sollten diese Medienkanäle durch breit angelegte Sensibilisierungskampagnen und die Förderung von Rollenvorbildern in den Zielgruppen genutzt werden.

\section{Textfeld 2.3. Ein Beispiel für eine Kontaktaufnahme über die Medien}

Ein gutes Beispiel für eine Kontaktaufnahme unter Nutzung der Medien ist Going for Growth in Irland, das darauf abzielt, Frauen dazu zu ermutigen, ihr Unternehmen wachsen zu lassen. Das Programm nutzt ein Netzwerk aus Champions, um bei Frauen für ein wachstumsstarkes Unternehmertum zu werben, und setzt Medienkampagnen effektiv ein, um Unternehmerinnen zu erreichen.

Zwar ist der Einsatz des angemessensten Kommunikationsmediums wichtig, doch auch auf die Botschaft, die vermittelt wird, ist zu achten. Die Botschaften müssen auf die spezifischen Zielgruppen zugeschnitten sein und für diese differenziert werden, um die Chancen zu erhöhen, mit ihnen Verbindung aufzunehmen. So unterscheiden sich zum Beispiel die Botschaften an potenzielle Jungunternehmer von denen an Frauen oder Ältere. Darüber hinaus muss die Kontaktaufnahme zu Gruppen von Zuwanderern und ethnischen Minderheiten unter Umständen in mehreren Sprachen erfolgen.

\section{Textfeld 2.4. Beispiele für Kontaktaufnahmen durch Mundpropaganda und Veranstaltungen}

In diesem Kompendium ist eine Reihe von Beispielen für eine zielgerichtete Kontaktarbeit enthalten. In Dänemark nutzt das Projekt Geschäftscoaching für ethnische Minderheiten in der Stadt Vejle alle zwei Monate stattfindende Kontaktaufnahmetreffen in verschiedenen Sprachen, um Unternehmer aus verschiedenen ethnischen Gemeinschaften zu erreichen. Die Treffen werden in der Regel in Zusammenarbeit mit lokalen Wirtschaftsverbänden ausgerichtet. Auch die Mundpropaganda spielt eine wichtige Rolle bei der Förderung des Coaching-Programms.

DreamStart, ein integriertes Programm zur Unterstützung des Unternehmertums für arbeitslose junge Menschen in Belgien, bemühte sich anfangs wenig um Kontaktaufnahmen. Stattdessen stützte es sich auf Weitervermittlungen. Das Ergebnis war jedoch ein Strom potenzieller Klienten, die alle dasselbe Profil und dieselben Ideen hatten. Zur Ausweitung seines Klientenstamms gibt DreamStart nun Präsentationen bei Veranstaltungen, richtet Kontaktaufnahmetreffen aus und verteilt Drucksachen über die Unternehmensagentur. 


\section{Nutzung von Wettbewerbsmechanismen, um Menschen mit Erfolgspotenzial zielgerichtet eine intensive Unterstützung zu leisten}

Viele Programme, die nachweislich Auswirkungen haben, verwenden Auswahlkriterien, um Teilnehmerauszuwählen.Zu den gängigenAnsätzen gehörtdieNutzungvonWettbewerbsmechanismen (z. B. eines Wettbewerbs um den besten Geschäftsplan), um diejenigen auszuwählen, die mit der höchsten Wahrscheinlichkeit bei der Unternehmensgründung erfolgreich sein werden. Die Nutzung strikter Auswahlkriterien sorgt für eine Verbesserung der Leistungskennzahlen des Programms (z. B. der Überlebensraten der Unternehmen der Teilnehmer). Vor allem kostspielige Programme, zum Beispiel persönliches Coaching oder Startkapitalprogramme, nutzen solche strikten Auswahlmechanismen, um zu gewährleisten, dass die Programmergebnisse im Einklang mit den Kosten stehen.

\section{Textfeld 2.5. Ein Beispiel für die Nutzung von Wettbewerbsmechanismen zur Vergabe von Unterstützungsleistungen}

Reifer Unternehmer in Polen ist ein Beispiel für die Integration eines Wettbewerbselements, um Unterstützungsleistungen auf die Teilnehmer auszurichten, die gute Erfolgschancen haben. Für den Schulungsbestandteil wurden die Teilnehmer durch schriftliche Bewerbungen und kurze Gespräche ausgewählt. Die Teilnehmer, die mindestens $80 \%$ der Schulung abschlossen, konnten an einem Wettbewerb für eine einmalige Beihilfe und eine Überbrückungshilfe für sechs Monate teilnehmen. Diese finanzielle Unterstützung wurde von einem Komitee vergeben, das aus Vertretern lokaler Arbeitgeberorganisationen bestand.

Mit der Nutzung von Wettbewerbsmechanismen zur Auswahl von Teilnehmern sind jedoch auch Risiken verbunden, die politische Entscheidungsträger berücksichtigen müssen. Da durch Wettbewerbsmechanismen diejenigen ausgewählt werden, die die größten Erfolgschancen haben, besteht die Gefahr, dass denjenigen mit öffentlichen Unterstützungsleistungen geholfen wird, die diese gar nicht benötigen (Kosten aufgrund von Mitnahmeeffekten). Außerdem schließen diese zur Auswahl eingesetzten Wettbewerbsmechanismen fast immer die am stärksten benachteiligten Menschen aus und untergraben so die Ziele der Politiken für ein integrationsförderndes Unternehmertum. Daher müssen die zur Auswahl der Teilnehmer verwendeten Kriterien neben Fertigkeiten und Fähigkeiten auch der Motivation und den Zielen der Intervention Rechnung tragen.

Alternativ kann ein gestuftes Unterstützungsverfahren eingesetzt werden, um die Teilnehmer zu filtern. Kostengünstige Unterstützungsleistungen (z. B. eine grundlegende Unternehmerausbildung) können einer breiten Basis potenzieller Klienten angeboten werden, während den Teilnehmern stufenweise mit jedem erfolgreich abgeschlossenen Schritt eine intensivere Unterstützung geleistet wird.

\section{Textfeld 2.6. Beispiele für Filtermechanismen}

Bei Politiken und Programmen für ein integrationsförderndes Unternehmertum wird weithin der „Trichter"-Ansatz verwendet. Hierfür sind mehrere Beispiele in diesem Kompendium enthalten. Die Maßnahme zur Aufnahme einer gewerblichen Tätigkeit oder selbstständigen Erwerbstätigkeit in Lettland bietet Unterstützungin zwei Phasen.Zunächstkönnen dieTeilnehmerbis zu 20 Unternehmensberatungstermine über sechs Wochen hinweg in Anspruch nehmen, die ihnen bei der Entwicklung eines Geschäftsplans helfen sollen. Anschließend können sie sich für die zweite Unterstützungsphase bewerben, in der sie zusätzliche Unternehmensberatung und finanzielle Beihilfen erhalten können. Damit sie diese zusätzliche Unterstützung in Anspruch nehmen können, werden ihre Geschäftspläne von Branchenexperten hinsichtlich der Einzigartigkeit des Produkts bzw. der Dienstleistung, des Innovationsgrads und der Größe des potenziellen Marktes bewertet.

BBZ in den Niederlanden bietet Arbeitslosen, die ein Unternehmen gründen, finanzielle Unterstützung, Schulungen und Wirtschaftsförderungsdienste. Eines der Merkmale dieses Programms ist der Umstand, dass die Teilnehmer abhängig von ihren Bedürfnissen in verschiedene Gruppen sortiert werden. Die verschiedenen Gruppen erhalten Unterstützungsleistungen unterschiedlicher Intensität. 


\section{Einsatz von qualifizierten Ausbildern und Beratern}

Politische Entscheidungsträger müssen die Qualität der geleisteten Unterstützungsdienste beachten. Ausbilder, Mentoren, Betreuer und Berater für Unternehmer sollten die Herausforderungen und Bedürfnisse der jeweiligen benachteiligten und unterrepräsentierten Gruppen, mit denen sie arbeiten, sehr genau verstehen. Darum ist es wichtig, dass die Ausbilder und Berater für die Arbeit mit der jeweiligen Zielgruppe geschult werden. Auch die Einsetzung von Menschen aus der Zielgruppe oder mit Erfahrungen mit der Zielgruppe ist von Bedeutung. Alternativ können die Agenturen, die die Programme umsetzen, mit privatwirtschaftlichen Organisationen zusammenarbeiten, um deren Erfahrungen und Fachwissen zu nutzen.

\section{Textfeld 2.7. Beispiele für die Zusammenarbeit mit Partnern bei der Bereitstellung von Schulungen}

Dieses Kompendium enthält mehrere Beispiele für öffentlich-private Partnerschaften. Eines davon ist der Förderungsfonds für das Unternehmertum in Litauen, der Mikrokreditfinanzierungen und Schulungen bietet. Verwaltet wird er von INVEGA, der für die Entwicklung kleiner und mittlerer Unternehmen zuständigen Agentur, in Zusammenarbeit mit dem Ministerium für soziale Sicherheit und Arbeit und dem Finanzministerium. Von zentraler Bedeutung für die Durchführung des Programms sind eine Reihe privatwirtschaftlicher Organisationen, einschließlich der Litauischen Zentralen Kreditgenossenschaft, anderen Kreditgenossenschaften und Schulungsorganisationen. INVEGA nutzt die Stärken jeder der privatwirtschaftlichen Organisationen für unterschiedliche Bestandteile des Programms.

Bei Unternehmertum funktioniert in Belgien handelt es sich um ein Coaching-Programm für Arbeitslose, die sich für das Unternehmertum interessieren. Es stützt sich auf eine enge Zusammenarbeit zwischen mehreren Organisationen, insbesondere der Union selbstständig Erwerbstätiger und KMU (UNIZO) (dem Hauptverwalter), der flämischen öffentlichen Arbeitsverwaltung für die Kontaktaufnahme mit den Arbeitslosen und einer Schulungsorganisation, die das Coaching durchführt.

In ähnlicher Weise führt Invitalia, Italiens Nationalagentur, die für die Verbesserung der Wettbewerbsfähigkeit Italiens verantwortlich ist, mehrere Unternehmensgründungsprogramme durch. $\mathrm{Zu}$ den verfügbaren Unterstützungsleistungen gehören Finanzierung, Schulungen, Coaching und Mentoring. Die Agentur nutzt lokale und regionale Partnerschaften, um potenzielle Klienten zu erreichen und hochwertige Unterstützungsleistungen bereitzustellen, die für den lokalen Kontext relevant sind.

Eine Möglichkeit, um die Qualität der Unterstützung für Unternehmensgründungen und die selbstständige Erwerbstätigkeit sicherzustellen, ist der Aufbau eines Akkreditierungssystems, mit dem die Erfahrungen und Fähigkeiten derjenigen geprüft werden, die die Unterstützung bereitstellen. Außerdem können öffentliche Mittel für Unterstützungsleistungen für Unternehmensgründungen und die selbstständige Erwerbstätigkeit an die Anforderung geknüpft werden, dass geschulte und qualifizierte Wirtschaftstrainer und -berater eingesetzt werden.

\section{Textfeld 2.8. Ein Beispiel für die Akkreditierung von Organisationen zur Unternehmensförderung}

Ein Beispiel für einen Ansatz zur Sicherstellung eines hochwertigen Wirtschaftsförderungsdienstes bietet Prowess, ein Mitgliedernetzwerk bestehend aus Organisationen von frauenfreundlichen Dienstleistern zur Unterstützung von Unternehmensgründungen im gesamten Vereinigten Königreich. Eine der Schlüsselinitiativen des Netzwerks war die Vergabe des Prowess Flagship Award, mit der frauenfreundliche Dienstleistungen zur Unternehmensförderung anerkannt werden sollten. 


\section{Aus Erfahrungen lernen}

Evaluierungen sind ein wichtiges Werkzeug zur Unterstützung der Gestaltung und Umsetzung von Politiken und Programmen für ein integrationsförderndes Unternehmertum. Sie können so gestaltet sein, dass sie politische Maßnahmen hinsichtlich einer Reihe von zentralen Erfolgskriterien bewerten (z. B. Relevanz, Wirksamkeit, Effizienz, Auswirkungen, Nachhaltigkeit) und auf der Grundlage der aus dieser Bewertung gezogenen Schlüsse Bereiche mit Verbesserungspotenzial ermitteln (siehe Tabelle 2.1). Die Ergebnisse dieser Evaluierungen sollten dann als Rückmeldung in die Politikgestaltung und die Bereitstellungsverfahren zurückgegeben werden.

\section{Tabelle 2.1. Zentrale Evaluierungskriterien}

\begin{tabular}{|c|c|c|}
\hline Kennzahlen & Definition & Beispielfragen \\
\hline \multirow[t]{2}{*}{ Relevanz } & \multirow{2}{*}{$\begin{array}{l}\text { Das Ausmaß, in dem sich die Aktivität für die Prioritäten und } \\
\text { Politiken der Zielgruppe, -empfänger und -regierung eignet } \\
\text { (Ziele im Vergleich zu Bedürfnissen). }\end{array}$} & $\begin{array}{l}\text { Stellt die Finanzierung immer noch ein Hindernis für das } \\
\text { weibliche Unternehmertum dar? }\end{array}$ \\
\hline & & $\begin{array}{l}\text { Sorgen Änderungen der Vorschriften im Zusammenhang } \\
\text { mit Leistungen, die Menschen mit Behinderungen beziehen, } \\
\text { dafür, dass eine Unternehmensgründung für diese Menschen } \\
\text { unattraktiv wird? }\end{array}$ \\
\hline \multirow[t]{2}{*}{ Wirksamkeit } & \multirow{2}{*}{$\begin{array}{l}\text { Das Ausmaß, in dem die Ziele der Intervention unter } \\
\text { Berücksichtigung ihrer relativen Wichtigkeit (Ergebnisse im } \\
\text { Vergleich zu Zielen) erreicht werden oder damit zu rechnen ist, } \\
\text { dass sie erreicht werden. }\end{array}$} & $\begin{array}{l}\text { Wurde die angestrebte Anzahl von durch junge Menschen } \\
\text { gegründeten Unternehmen erreicht? }\end{array}$ \\
\hline & & Haben diese Unternehmen zwei Jahre lang überlebt? \\
\hline \multirow[t]{4}{*}{ Effizienz } & \multirow{4}{*}{$\begin{array}{l}\text { Die Ergebnisse im Vergleich zu den Eingaben. Hierbei handelt } \\
\text { es sich um einen Wirtschaftsbegriff, der besagt, dass die } \\
\text { Intervention die am wenigsten kostspieligen Ressourcen zur } \\
\text { Erreichung der angestrebten Ergebnisse verwendet (Input im } \\
\text { Vergleich zu Output). }\end{array}$} & Wie hoch waren die Kosten pro beratene Person? \\
\hline & & $\begin{array}{l}\text { Wie hoch waren die Kosten pro geschaffenen Arbeitsplatz für } \\
\text { einen Roma? }\end{array}$ \\
\hline & & $\begin{array}{l}\text { Wie hoch lag der prozentuale Anteil von Klienten, die aus der } \\
\text { Zielgruppe stammten? }\end{array}$ \\
\hline & & Gab es effizientere Methoden zur Umsetzung der Maßnahme? \\
\hline \multirow[t]{3}{*}{ Auswirkungen } & \multirow{3}{*}{$\begin{array}{l}\text { Die positiven und negativen Veränderungen, die direkt oder } \\
\text { indirekt, beabsichtigt oder unbeabsichtigt durch eine politische } \\
\text { Intervention herbeigeführt werden (Ziele im Vergleich zu } \\
\text { Ergebnissen). }\end{array}$} & $\begin{array}{l}\text { Liegen die Rate der Unternehmensinhaber und die Rate der } \\
\text { selbstständig Erwerbstätigen in der Zielgruppe nun höher? }\end{array}$ \\
\hline & & Liegt die Beschäftigungsquote der Zielgruppe nun höher? \\
\hline & & Hat sich die soziale Integration erhöht? \\
\hline \multirow[t]{3}{*}{ Nachhaltigkeit } & \multirow[t]{3}{*}{$\begin{array}{l}\text { Ob der Nutzen einer Aktivität wahrscheinlich fortbesteht, } \\
\text { nachdem die Finanzierung entzogen wird. }\end{array}$} & $\begin{array}{l}\text { Wird sich das für ältere Unternehmer eingerichtete } \\
\text { Mikrokreditprogramm selbst tragen? }\end{array}$ \\
\hline & & $\begin{array}{l}\text { Ist das Beratungszentrum in der Lage, die Fähigkeiten } \\
\text { beizubehalten, die es entwickelt hat? }\end{array}$ \\
\hline & & Wird weitere öffentliche Unterstützung benötigt? \\
\hline
\end{tabular}

Quelle: OECD/Europäische Kommission (2013), „Kurzdossier zur Evaluierung von Maßnahmen für ein integrationsförderndes Unternehmertum", EU: Luxemburg. http://ec.europa.eu/social/BlobServlet?docId=11086\&langId=de

Es ist wichtig zu berücksichtigen, wie Evaluierungen im politischen Verfahren eingesetzt werden. Auch Ex-ante-Evaluierungen sollten vorgenommen werden, um die Relevanz und Kohärenz der vorgeschlagenen politischen Maßnahmen zu bewerten. In diesem Stadium sind Überwachungs- und Evaluierungsindikatoren zu ermitteln, ein logischer Rahmen ist festzulegen, um explizit darzulegen, wie die politische Maßnahme einen Nutzen bringen soll, und es ist ein Evaluierungsplan zu entwerfen, der zeigt, wie die Maßnahme während und nach dem Umsetzungszeitraum bewertet wird. Gleichfalls von Bedeutung während dieses Prozesses ist die Berücksichtigung der Ergebnisse von Evaluierungen ähnlicher Programme in der Region, im Land oder andernorts.

Es kann auch wertvoll sein, ein Pilotprojekt einzurichten und zu evaluieren, um einschätzen zu können, ob eine vorgeschlagene Programmlogik dem Praxistest standhält, ob sich das Programm als relevant, wirksam und effizient erweist, und um herauszufinden, welche Arten von Problemen sich bei dem Versuch, die Initiative auszuweiten oder zu etablieren, ergeben könnten. 


\section{Textfeld 2.9. Beispiele für das Lernen aus Pilotprojekten}

Eine Reihe von Beispielen, die in diesem Kompendium vorgestellt werden, haben Pilotprojekte durchgeführt. Unternehmerisch in die Geschäftswelt in Slowenien ist ein Beispiel, bei dem im kleinen Umfang mit einer Idee experimentiert wurde, die dann ausgeweitet wurde, sobald sie Erfolge nachweisen konnte. Bei diesem Projekt werden arbeitslose junge Menschen als öffentliche Bedienstete eingestellt, die eine Unternehmerausbildung und Coaching erhalten, statt zu arbeiten. Im Wesentlichen funktioniert dies wie eine Zuwendung. Das Projekt wurde von 2009 bis 2012 in der Region Zasavje getestet, 2013 national umgesetzt und 2014 ausgeweitet.

Das Projekt Startrampen für Beschäftigung und Unternehmertum in Spanien bietet Gruppen- und EinzelCoachings für Arbeitslose an, die ein Unternehmen gründen oder eine Beschäftigung finden möchten. Es wurde nicht als Pilotprojekt getestet, sondern ist einem anderen Projekt nachempfunden, das arbeitslosen jungen Menschen in mehr als 17 Ländern handwerkliche Fähigkeiten vermittelte.

Ex-post-Evaluierungen werden vorgenommen, sobald eine politische Maßnahme ausreichend lange durchgeführt wurde, um Resultate hervorzubringen. Einer der wesentlichen Aspekte der Bewertung von Wirkungen hängt mit der Ermittlung und Berücksichtigung des Kontrafaktischen zusammen, indem zum Beispiel die Auswirkungen auf Kontrollgruppen von Nichtbegünstigten verfolgt und mit den Teilnehmern, die eine politische Unterstützung erhalten, verglichen werden.

Evaluierungen könnten recht technisch werden, wenn moderne Methoden wie kontrafaktische Techniken zum Einsatz kommen. Dies sollte uns jedoch nicht daran hindern, Nutzen aus Evaluierungen $\mathrm{zu}$ ziehen und dadurch zu erfahren, welche politischen Ansätze für bestimmte Zielgruppen am besten funktionieren und wie sie verbessert werden können. Entscheidend ist, dass politische Entscheidungsträger die Hauptprobleme kennen, sodass sie bei Experten geeignete Evaluierungen in Auftrag geben können und bei der Schaffung von Kulturen mitwirken, wobei Politikgestaltung bzw. -umsetzung und Evaluierungen miteinander einhergehen.

\section{Textfeld 2.10. Beispiele für umfassende Evaluierungen}

Dieses Kompendium enthält eine Reihe von Beispielen für Programme, die umfassend evaluiert wurden. Das Überbrückungsgeld in Deutschland ist ein groß angelegtes Unterstützungsprogramm für Arbeitslose, die sich für eine selbstständige Erwerbstätigkeit interessieren. Der große Umfang des Programms und die Fülle anVerwaltungsinformationen und -daten haben eine umfassende Evaluierung ermöglicht. Dies hat politischen Entscheidungsträgern Aufschluss über wirksame Maßnahmen und über Bereiche gegeben, in denen das Programm verbessert werden kann.

Auch die Beihilfe zur Unternehmensgründung in Finnland wurde regelmäßig evaluiert. Bei Beihilfen zur Unternehmensgründung für Arbeitslose beschäftigen die politischen Entscheidungsträger zwei Hauptanliegen: das Ausmaß, in dem öffentliche Mittel von denjenigen in Anspruch genommen werden, die ohne die Beihilfe ein Unternehmen gegründet hätten, und das Ausmaß, in dem diejenigen, die öffentliche Unterstützung erhalten, andere aus dem Markt verdrängen. Ausführliche Evaluierungen können diese Auswirkungen offenlegen und ermöglichen es, die Maßnahme gezielter durchzuführen. Finnland ist in dieser Hinsicht führend.

Ziel des Unternehmensgründungsprogramms in Österreich ist es, Arbeitslose bei der Unternehmensgründung zu unterstützen. Anfangs konzentrierte sich das Programm ausschließlich auf die Unterstützung vor der Unternehmensgründung, doch deuteten Evaluierungen darauf hin, dass die fehlende Unterstützung im Anschluss an die Unternehmensgründung der Hauptgrund für die beobachtete hohe Misserfolgsquote war. Daher wurde das Projekt angepasst: Die Teilnehmer können nun bis zu zwei Jahre nach der Unternehmensgründung Unternehmensberatung in Anspruch nehmen. 
Tabelle 2.2. Übersicht der vorgestellten Politiken und Programme

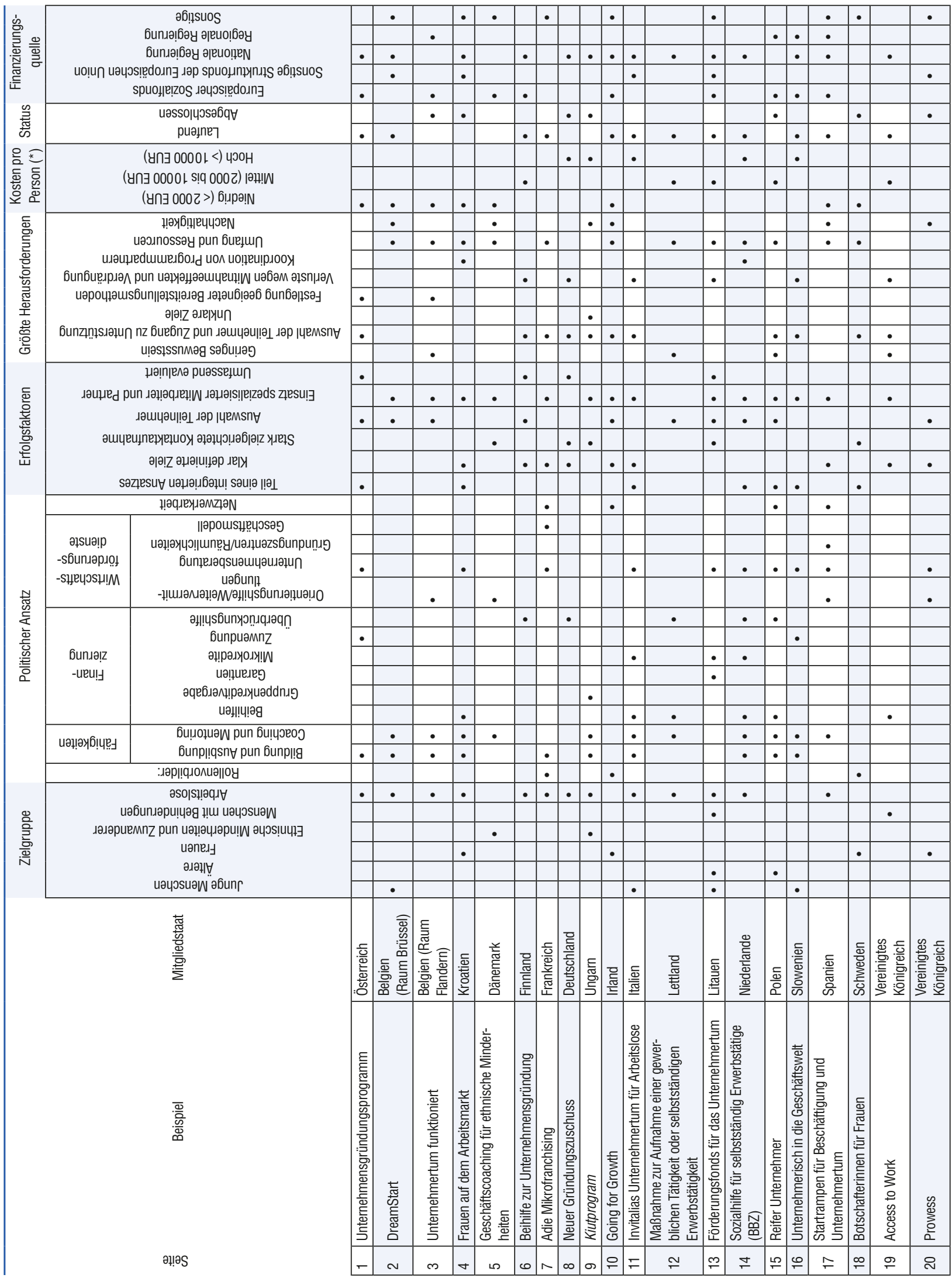




\section{Literatur}

Berichte „Die fehlenden Unternehmer“

OECD/EuropäischeKommission(2015),DiefehlendenUnternehmer 2015:Politikenfüreinintegrationsförderndes Unternehmertum in Europa, OECD Publishing. http://dx.doi.org/10.1787/9789264249943-de

OECD/EuropäischeKommission(2014),DiefehlendenUnternehmer 2014:Politikenfüreinintegrationsförderndes Unternehmertum in Europa, OECD Publishing. http://dx.doi.org/10.1787/9789264230897-de

OECD/Europäische Kommission (2013), Die fehlenden Unternehmer: Politiken für ein integrationsförderndes Unternehmertum in Europa, OECD Publishing. http://dx.doi.org/10.1787/9789264188419-de

\section{Kurzdossiers zum integrationsfördernden Unternehmertum}

OECD/Europäische Kommission (2015), „Policy Brief on Sustaining Self-employment“, OECD Publishing, Paris, und EU Publishing, Luxemburg. http://dx.doi.org/10.1787/5jrtpbxh2v21-en

OECD/Europäische Kommission (2015), „Kurzdossier zum informellen Unternehmertum“, OECD Publishing, Paris, und EU Publishing, Luxemburg. ec.europa.eu/social/BlobServlet?docId=13791\&lan gId $=$ de

OECD/Europäische Kommission (2015), „Kurzdossier zu Netzwerken für ein integrationsförderndes Unternehmertum“, OECD Publishing, Paris, und EU Publishing, Luxemburg. ec.europa.eu/social/ BlobServlet? docId=13739\&langId=de

OECD/Europäische Kommission (2014), „Kurzdossier zum Unternehmertum für Menschen mit Behinderung“, OECD Publishing, Paris, und EU Publishing, Luxemburg. ec.europa.eu/social/ BlobServlet? docId=12561\&langId=de

OECD/Europäische Kommission (2014), „Kurzdossier zum Zugang zu Gründungsfinanzierung für ein integrationsförderndes Unternehmertum“, OECD Publishing, Paris, und EU Publishing, Luxemburg. ec.europa.eu/social/BlobServlet?docId=11499\&langId=de

OECD/Europäische Kommission (2013), „Kurzdossier zur Evaluierung von Maßnahmen für ein integrationsförderndes Unternehmertum“, OECD Publishing, Paris, und EU Publishing, Luxemburg. ec.europa.eu/social/BlobServlet?docId=11086\&langId=de

OECD/Europäische Kommission (2013), „Kurzdossier zu sozialem Unternehmertum“, OECD Publishing, Paris, und EU Publishing, Luxemburg. ec.europa.eu/social/BlobServlet?docId=13503\&langId=de

OECD/Europäische Kommission (2012), „Kurzdossier zur unternehmerischen Initiative älterer Menschen“, OECD Publishing, Paris, und EU Publishing, Luxemburg. ec.europa.eu/social/ BlobServlet? docId $=9644 \&$ langId $=$ de

OECD/Europäische Kommission (2012), „Kurzdossier zur unternehmerischen Initiative junger Menschen“, OECD Publishing, Paris, und EU Publishing, Luxemburg. http://ec.europa.eu/social/ BlobServlet? docId=8759\&langId=de 


\section{Anhang: Zusammenfassende Tabellen}




\section{Österreich}

\begin{tabular}{|c|c|}
\hline \multicolumn{2}{|r|}{ Unternehmensgründungsprogramm } \\
\hline Zielgruppe & Arbeitslose \\
\hline Geleistete Unterstützung & Schulungen, Unternehmensberatung, Zuwendungen \\
\hline Ziel & $\begin{array}{l}\text { Ziel des Programms ist die Reduzierung der Arbeitslosigkeit durch die Unterstützung Arbeitsloser bei der Gründung eines } \\
\text { eigenen Unternehmens. }\end{array}$ \\
\hline Status & 1995 gestartet; laufend. \\
\hline \multirow[t]{3}{*}{ Kurzprofil des Programms } & $\begin{array}{l}\text { Das Unternehmensgründungsprogramm (UGP) des Arbeitsmarktservice Österreich (AMS) bietet ein integriertes } \\
\text { Unterstützungspaket für Arbeitslose, die sich für die Aufnahme einer selbstständigen Erwerbstätigkeit interessieren. }\end{array}$ \\
\hline & $\begin{array}{l}\text { Das Unterstützungsprogramm ist in vier Phasen aufgebaut: Die Klärungsphase besteht aus einem Aufnahmeprozess, bei dem die } \\
\text { Realisierbarkeit der Geschäftsidee geprüft wird und Bedürfnisse des Unternehmers ermittelt werden. In der Vorbereitungsphase } \\
\text { findet der größte Teil des Unternehmensgründungsprogramms statt, unter anderem individuelle Unternehmensberatung und } \\
\text { Unternehmensgründungsworkshops. In der Umsetzungsphase starten die Teilnehmer ihr Unternehmen. Sie können zusätzliche } \\
\text { Beratungsangebote in Anspruch nehmen und erhalten zwei Monate lang Zuwendungen zur Unternehmensgründung. Die } \\
\text { Zuwendungen entsprechen den Leistungen, die sie in Form ihrer Arbeitslosenunterstützung bezogen hätten, zuzüglich eines } \\
\text { Betrags, um ihre Sozialversicherungsbeiträge zu decken. Nach der Gründung des Unternehmens sieht eine Nachbereitungsphase } \\
\text { eine „Kontrolle“ der neuen Unternehmen vor. Im Anschluss an die Unternehmensgründung wird zwei Jahre lang eine zusätzliche } \\
\text { Beratung angeboten. }\end{array}$ \\
\hline & Das Programm wird durch die regionalen AMS-Büros verwaltet, aber von externen Beratern durchgeführt. \\
\hline Hintergrund & $\begin{array}{l}\text { Arbeitslose erhalten nur dann eine Arbeitslosenunterstützung, wenn sie gemeldet sind und für eine Beschäftigung zur Verfügung } \\
\text { stehen. Diejenigen, die ein Unternehmen gründen, müssen auf ihre Arbeitslosenunterstützung verzichten, da sie nicht mehr } \\
\text { für eine Beschäftigung zur Verfügung stehen. Dies kann von einer selbstständigen Erwerbstätigkeit als Weg zurück in die } \\
\text { Beschäftigung abschrecken. }\end{array}$ \\
\hline Dauer der Unterstützung & $\begin{array}{l}\text { Schulungen und Unternehmensberatung werden für bis zu sechs Monate angeboten. Eine Verlängerung auf bis zu neun Monate } \\
\text { ist möglich. Die Zuwendungen stehen zwei Monate lang zur Verfügung. }\end{array}$ \\
\hline \multirow[t]{2}{*}{ Größte Herausforderungen } & $\begin{array}{l}\text { 1. Schaffung eines Gleichgewichts zwischen der Notwendigkeit, Teilnehmer auszuwählen, die gute Erfolgschancen haben, und } \\
\text { der Notwendigkeit, die am stärksten Benachteiligten zu unterstützen. }\end{array}$ \\
\hline & $\begin{array}{l}\text { 2. Sicherstellung der Kosteneffektivität des Programms, da es eine intensive individuelle Unterstützung sowie nicht } \\
\text { rückzahlungspflichtige finanzielle Zuwendungen bereitstellt. }\end{array}$ \\
\hline \multirow[t]{3}{*}{ Auswirkungen } & Von den 8683 Menschen, die 2013 an dem Programm teilnahmen, gründeten 5074 ein Unternehmen (58\%). \\
\hline & $\begin{array}{l}\text { Die Überlebensraten für unterstützte Unternehmen liegen bei } 89 \% \text { für ein Jahr, } 75 \% \text { für drei Jahre und } 64 \text { \% für fünf Jahre. } \\
\text { Diese Raten liegen etwas höher als die Überlebensraten der allgemeinen Unternehmerbevölkerung. }\end{array}$ \\
\hline & $\begin{array}{l}\text { Eine Evaluierung aus dem Jahr } 2006 \text { zeigt, dass die im Rahmen dieses Programms gegründeten Unternehmen netto 1,26 neue } \\
\text { Arbeitsplätze zusätzlich zu dem des Unternehmers geschaffen haben. }\end{array}$ \\
\hline Durchschnittliche jährliche Kosten & 5 Millionen EUR für Beratung und Schulungen; 12 Millionen EUR für Zuwendungen \\
\hline Kosten pro Teilnehmer (") & 1960 EUR \\
\hline Finanzierungsquellen & $\begin{array}{l}\text { Das Programm wird derzeit durch die nationale Regierung finanziert; bis } 2006 \text { erhielt es Mittel aus dem Europäischen } \\
\text { Sozialfonds. }\end{array}$ \\
\hline
\end{tabular}

(*) Beim Vergleich der Kosten pro Teilnehmer der verschiedenen Programme sollten die Dauer der Programme und die Unterschiede in den angebotenen Dienstleistungen beachtet werden. 


\section{Belgien (Raum Brüssel)}

\begin{tabular}{|c|c|}
\hline \multicolumn{2}{|r|}{ DreamStart Facility } \\
\hline Zielgruppe & Arbeitslose junge Menschen (18- bis 30-Jährige) \\
\hline Geleistete Unterstützung & Schulungen, Coaching und Mentoring \\
\hline Ziel & $\begin{array}{l}\text { Die Unterstützung arbeitsloser junger Menschen bei der Entwicklung einer Geschäftsidee und der Erstellung eines } \\
\text { Geschäftsplans, damit sie ihr eigenes Unternehmen gründen können. }\end{array}$ \\
\hline Status & 2013 gestartet; laufend. \\
\hline \multirow[t]{2}{*}{ Kurzprofil des Programms } & $\begin{array}{l}\text { Das Projekt versteht sich als Kurs zur Geschäftsplanerstellung. Die Teilnehmer werden durch Aufnahmegespräche ausgewählt, in } \\
\text { denen ihre Geschäftsidee, ihr Antrieb und ihre Erfolgschancen beurteilt werden. }\end{array}$ \\
\hline & $\begin{array}{l}\text { Während eines zweimonatigen Zeitraums kommen die Teilnehmer drei ganze Tage in der Woche zusammen, um mit } \\
\text { Unternehmern, Start-up-Experten und anderen potenziellen Unternehmern zusammen zu lernen und zu interagieren. In diesen } \\
\text { zwei Monaten erhalten die Teilnehmer Schulungen und Beratung von freiwilligen Experten aus dem privatwirtschaftlichen und } \\
\text { öffentlichen Sektor sowie aus dem Finanzsektor. Die Teilnehmer arbeiten in diesem Zeitraum gemeinsam an der Entwicklung } \\
\text { ihrer Geschäftspläne. Es ist jedem Einzelnen überlassen, seinen Plan umzusetzen und sein eigenes Unternehmen zu gründen. } \\
\text { Eine Finanzierung und sonstige Unterstützungsleistungen für die Unternehmensgründung gehören zwar nicht offiziell zu diesem } \\
\text { Unterstützungspaket, doch haben die Teilnehmer häufig Zugang zu Mikrokrediten durch MicroStart, eine Schwesterorganisation } \\
\text { von DreamStart. }\end{array}$ \\
\hline Hintergrund & $\begin{array}{l}\text { Der Raum Brüssel hat mit einer hohen Jugendarbeitslosigkeit zu kämpfen, und es wird für die öffentlichen Arbeitsverwaltungen } \\
\text { immer schwieriger, für ihre Klienten eine Beschäftigung zu finden. Eine selbstständige Erwerbstätigkeit ist eine Möglichkeit, wie } \\
\text { einige der arbeitslosen jungen Menschen wieder an den Arbeitsmarkt zurückkehren können. }\end{array}$ \\
\hline Dauer der Unterstützung & Schulungen, Coaching und Mentoring werden zwei Monate lang angeboten. \\
\hline \multirow[t]{4}{*}{ Größte Herausforderungen } & $\begin{array}{l}\text { 1. Das Projekt stützt sich in hohem Maße auf Freiwillige. Dies ist hinderlich bei der Erweiterung des Umfangs der angebotenen } \\
\text { Leistungen. }\end{array}$ \\
\hline & $\begin{array}{l}\text { 2. Die Sicherung ausreichender finanzieller Ressourcen stellt ein Problem dar. Das Projekt wird durch öffentliche Mittel, } \\
\text { Spenden aus der Privatwirtschaft und Sacheinlagen finanziert. Eine Gebühr wird nicht erhoben, da dies potenzielle Teilnehmer } \\
\text { abschrecken könnte. }\end{array}$ \\
\hline & $\begin{array}{l}\text { 3. Das Projekt konzentriert seine Bemühungen auf die Phase vor der Unternehmensgründung, doch die Teilnehmer benötigen } \\
\text { wahrscheinlich auch Unterstützung nach der Unternehmensgründung. Man bemüht sich um mehr und engere Verbindungen zu } \\
\text { Anbietern von Wirtschaftsförderungsdiensten. }\end{array}$ \\
\hline & 4. Die Teilnehmer benötigen mehr Unterstützung beim Zugang zu Finanzierung, als derzeit angeboten wird. \\
\hline \multirow[t]{2}{*}{ Auswirkungen } & $\begin{array}{l}\text { Über drei Projektzyklen hinweg sind bislang } 36 \text { junge Menschen unterstützt worden. } 60 \% \text { derjenigen, die das Programm } \\
\text { abschlossen, gründeten innerhalb eines Jahres ein Unternehmen. Ein Drittel von ihnen war im eigenen Unternehmen in Vollzeit } \\
\text { tätig, und zwei Drittel kombinierten ihr Unternehmen mit einer abhängigen Erwerbstätigkeit. }\end{array}$ \\
\hline & Viele Teilnehmer kamen nach dem Programm in eigenen inoffiziellen Gruppen von Gleichgestellten zusammen. \\
\hline Durchschnittliche jährliche Kosten & 72000 EUR für drei Zyklen (ausgenommen 70000 EUR an Sacheinlagen von Freiwilligen) \\
\hline Kosten pro Teilnehmer (*) & 2000 EUR \\
\hline Finanzierungsquellen & Öffentliche Mittel (42\%), Spenden aus der Privatwirtschaft (7\%) und Sacheinlagen (51\%). \\
\hline
\end{tabular}

$\left(^{*}\right)$ Beim Vergleich der Kosten pro Teilnehmer der verschiedenen Programme sollten die Dauer der Programme und die Unterschiede in den angebotenen Dienstleistungen beachtet werden. 


\section{Belgien (Flandern)}

\begin{tabular}{|c|c|}
\hline \multicolumn{2}{|r|}{ Unternehmertum funktioniert } \\
\hline Zielgruppe & Arbeitslose \\
\hline Geleistete Unterstützung & Coaching \\
\hline Ziel & Unterstützung von Arbeitslosen bei der Gründung eines Unternehmens. \\
\hline Status & 2008-2012; abgeschlossen. \\
\hline \multirow[t]{4}{*}{ Kurzprofil des Programms } & $\begin{array}{l}\text { „Unternehmertum funktioniert“ war ein Unternehmercoaching-Programm für Arbeitsuchende. Es handelte sich dabei um eine } \\
\text { Partnerschaft zwischen UNIZO (der Union selbstständig Erwerbstätiger und KMU), der VDAB (der flämischen öffentlichen } \\
\text { Arbeitsverwaltung) und Syntra (dem Netzwerk für Unternehmerausbildung), die durch den Europäischen Sozialfonds und die } \\
\text { VDAB finanziell unterstützt wurde. }\end{array}$ \\
\hline & $\begin{array}{l}\text { Vor der Zulassung in das Programm mussten interessierte Arbeitslose ein Screening durchlaufen. Externe Geschäftsexperten } \\
\text { identifizierten mittels des Selbstbewertungsinstruments „Entre Mirror“ die Kandidaten, die eine geeignete Idee und angemessene } \\
\text { Kompetenzen für eine selbstständige Erwerbstätigkeit hatten. }\end{array}$ \\
\hline & $\begin{array}{l}\text { Die erste Unterstützungsphase dauerte bis zu sechs Monate. Jeder Teilnehmer durchlief eine ausführliche Fähigkeits- und } \\
\text { Bedarfsanalyse, nahm an Schulungen teil und bekam einen Betreuer zugeteilt. Ziel der ersten Phase war es, einen Aktionsplan } \\
\text { für die Unternehmensgründung zu entwickeln und ein betriebswirtschaftliches Zertifikat (das in Flandern gefordert wird) zu } \\
\text { erlangen. }\end{array}$ \\
\hline & $\begin{array}{l}\text { In der zweiten Phase, die ebenfalls höchstens sechs Monate dauerte, erfolgte ein intensives Coaching, während die Teilnehmer } \\
\text { ihre Geschäftspläne umsetzten. Auch andere Aspekte wurden behandelt, etwa die Beantragung von Darlehen, die Suche nach } \\
\text { Gewerberäumen, die Kontaktaufnahme mit Lieferanten und potenziellen Kunden und die Entwicklung einer Marktstrategie. Den } \\
\text { Teilnehmern wurden zudem Workshops und Informationssitzungen zu betriebswirtschaftlichen Fallstricken angeboten. }\end{array}$ \\
\hline Hintergrund & $\begin{array}{l}\text { Das Programm wurde ins Leben gerufen, um zwei Hindernisse anzugehen, vor denen Arbeitslose im Rahmen der } \\
\text { Unternehmensgründung stehen. Erstens mangelt es Arbeitslosen im Allgemeinen an Motivation und Fähigkeiten für eine } \\
\text { selbstständige Erwerbstätigkeit. Zweitens waren die Arbeitsverwaltungen in Flandern Mitte der 2000er-Jahre fragmentiert, und es } \\
\text { gab wenig Unterstützung für Arbeitslose, die sich für eine selbstständige Erwerbstätigkeit interessierten. }\end{array}$ \\
\hline Dauer der Unterstützung & $\begin{array}{l}\text { Die zwei Phasen bestehend aus Coaching und Schulungen dauerten jeweils bis zu sechs Monate. Teilnehmer ohne } \\
\text { betriebswirtschaftliches Zertifikat waren verpflichtet, einen 132-stündigen Kurs zu absolvieren (außerhalb dieses Programms). }\end{array}$ \\
\hline \multirow[t]{3}{*}{ Größte Herausforderungen } & $\begin{array}{l}\text { 1. Für „Unternehmertum funktioniert“ war es schwierig, einige der Zielklienten zu erreichen, zum Beispiel Erwerbsunfähige und } \\
\text { ethnische Minderheitengruppen. Da alle Schulungskurse auf Niederländisch abgehalten wurden, stellte die Sprache für viele } \\
\text { Kandidaten eine erhebliche Hürde dar. }\end{array}$ \\
\hline & $\begin{array}{l}\text { 2. Eine zweite Herausforderung bestand darin, mit einem Team von nur fünf Betreuern ein professionelles Coaching für } \\
650 \text { Teilnehmer bereitzustellen. }\end{array}$ \\
\hline & $\begin{array}{l}\text { 3. Die richtige Balance zu finden zwischen der Bereitstellung eines strukturierten Programms zur Unterstützung von } \\
\text { Unternehmensgründungen und der Einräumung von ausreichend Flexibilität, um Kreativität zu fördern. }\end{array}$ \\
\hline Auswirkungen & $\begin{array}{l}2101 \text { Teilnehmer schlossen mindestens die erste Programmphase ab. 52,4\% der Teilnehmer gelang im Zeitraum 2011-2013 der } \\
\text { Übergang aus der Arbeitslosigkeit, davon waren } 90 \% \text { selbstständig erwerbstätig. }\end{array}$ \\
\hline Durchschnittliche jährliche Kosten & 1480000 EUR (für die letzte Durchführung) \\
\hline Kosten pro Teilnehmer (") & 1250 EUR (für die letzte Durchführung) \\
\hline Finanzierungsquellen & Regionale Regierung (51,7\%), Europäischer Sozialfonds (39,5\%) und sonstige $(8,8 \%)$. \\
\hline
\end{tabular}

(*) Beim Vergleich der Kosten pro Teilnehmer der verschiedenen Programme sollten die Dauer der Programme und die Unterschiede in den angebotenen Dienstleistungen beachtet werden. 


\section{Kroatien}

\begin{tabular}{|c|c|}
\hline \multicolumn{2}{|r|}{ Frauen auf dem Arbeitsmarkt } \\
\hline Zielgruppe & $\begin{array}{l}\text { Arbeitslose Frauen, insbesondere langzeitarbeitslose Frauen (d. h. länger als zwölf Monate ohne Beschäftigung) im Alter von } \\
\text { über } 40 \text { Jahren und Frauen in der öffentlichen Verwaltung }\end{array}$ \\
\hline Geleistete Unterstützung & Finanzierung (Beihilfen), Coaching und Mentoring, Schulungen, Unternehmensberatung \\
\hline Ziel & $\begin{array}{l}\text { Die Unterstützung arbeitsloser Frauen bei der Rückkehr in die Beschäftigung durch eine Unternehmensgründung und die } \\
\text { Stärkung der Kapazitäten der öffentlichen Verwaltung. }\end{array}$ \\
\hline Status & $\begin{array}{l}\text { Das Programm wurde } 2010 \text { aufgenommen und war } 2012 \text { abgeschlossen. Das Beihilfeprogramm lief von November } 2010 \text { bis } \\
\text { November 2011, das technische Unterstützungsprojekt von September } 2010 \text { bis März } 2012 .\end{array}$ \\
\hline \multirow[t]{2}{*}{ Kurzprofil des Programms } & $\begin{array}{l}\text { Bei „Frauen auf dem Arbeitsmarkt“ handelte es sich um ein zweigliedriges Projekt, das von der kroatischen Arbeitsverwaltung im } \\
\text { Rahmen des Instruments für Heranführungshilfe durchgeführt wurde. Ein Aspekt des Projekts war die Erbringung technischer } \\
\text { Unterstützung, deren Ziel die Nutzung bereits existierender Instrumente für Frauen zur Rückkehr auf den Arbeitsmarkt (z. B. } \\
\text { Schulungen) war. Das Hauptaugenmerk dieser Unterstützung lag auf der Suche nach einer Beschäftigung für Frauen (nicht auf } \\
\text { der Förderung der selbstständigen Erwerbstätigkeit). }\end{array}$ \\
\hline & $\begin{array}{l}\text { Zudem wurde ein Beihilfeprogramm eingerichtet, das verschiedene Projekte finanzierte, die Frauen bei ihrem Eintritt in } \\
\text { den Arbeitsmarkt unterstützen sollten. Nach der Prüfung von } 55 \text { Projektanträgen erhielten } 18 \text { Projekte Beihilfe für ihre } \\
\text { Umsetzung. Davon konzentrierten sich drei Projekte speziell auf unternehmerische Tätigkeiten: „Sei unternehmerisch - sei } \\
\text { wettbewerbsfähig“ in der Gespanschaft Brod-Posavina, „Schritt für Schritt in den Arbeitsmarkt“ in der Gespanschaft Vukovar- } \\
\text { Srijem und „MOTS - Mehr Möglichkeiten durch neue Fähigkeiten“ in Dubrovnik-Neretva. Diese Projekte stellten eine Reihe von } \\
\text { Unterstützungsleistungen bereit. }\end{array}$ \\
\hline Hintergrund & $\begin{array}{l}\text { Langzeitarbeitslose Frauen in Kroatien sind stark durch soziale Ausgrenzung bedroht. Das Unternehmertum wurde als ein } \\
\text { Weg betrachtet, um diese Herausforderung anzugehen und benachteiligte Frauen zu stärken. Zudem versuchte man mit dem } \\
\text { Programm, eine Lücke in der öffentlichen politischen Unterstützung für diese Frauen zu schließen. }\end{array}$ \\
\hline Dauer der Unterstützung & $\begin{array}{l}\text { Unternehmerprojekte wurden für zwölf Monate mit Beihilfen gefördert. Die technische Unterstützung wurde } 18 \text { Monate lang } \\
\text { bereitgestellt. }\end{array}$ \\
\hline \multirow[t]{3}{*}{ Größte Herausforderungen } & $\begin{array}{l}\text { 1. Die Organisationen, die die Beihilfen ausbezahlten, hatten Schwierigkeiten mit der Einhaltung der Anforderungen der } \\
\text { Europäischen Union sowie mit der Planung und Mittelverwaltung. }\end{array}$ \\
\hline & $\begin{array}{l}\text { 2. Für das technische Unterstützungsprojekt bestanden einige Herausforderungen, unter anderem hinter den Erwartungen } \\
\text { zurückbleibende Teilnehmerquoten (insbesondere seitens Frauen in öffentlichen Verwaltungsfunktionen). }\end{array}$ \\
\hline & $\begin{array}{l}\text { 3. In die Projekte waren keine Überwachung und Evaluierung integriert, sodass es schwierig ist, aus den gewonnen Erfahrungen } \\
\text { Erkenntnisse zu ziehen. }\end{array}$ \\
\hline \multirow[t]{5}{*}{ Auswirkungen } & Drei Beihilfeprojekte förderten direkt Unternehmensgründungen durch Frauen: \\
\hline & $\begin{array}{l}\text { Sei unternehmerisch - sei wettbewerbsfähig: Von } 415 \text { Frauen wurden } 55 \text { zur Teilnahme ausgewählt. Von diesen } 55 \text { gründeten } \\
13 \text { Frauen ein eigenes Unternehmen oder fanden eine anderweitige Beschäftigung. }\end{array}$ \\
\hline & $\begin{array}{l}\text { Schritt für Schritt in den Arbeitsmarkt: } 262 \text { Frauen nahmen an dem Programm teil. Von diesen gründeten zwei jeweils } \\
\text { ein eigenes Unternehmen (bei denen } 13 \text { andere Teilnehmerinnen beschäftigt wurden), drei Frauen gründeten gemeinsam } \\
\text { ein Unternehmen und } 27 \text { Frauen fanden eine anderweitige Beschäftigung. Insgesamt fanden } 45 \text { Frauen eine produktive } \\
\text { Beschäftigung verschiedener Art. }\end{array}$ \\
\hline & $\begin{array}{l}\text { MOTS - Mehr Möglichkeiten durch neue Fähigkeiten: } 239 \text { arbeitslose Frauen nahmen teil, } 59 \text { fanden eine Beschäftigung, neun } \\
\text { gründeten ein eigenes Unternehmen. }\end{array}$ \\
\hline & Ein weiteres Ergebnis war ein Kapazitätsaufbau in der öffentlichen Verwaltung. \\
\hline Durchschnittliche jährliche Kosten & $\begin{array}{l}\text { Beihilfeprogramm: } 1853606 \text { EUR (davon wurden } 309412 \text { EUR für die drei Unternehmerprojekte verwendet); technisches } \\
\text { Unterstützungsprojekt: } 581000 \text { EUR }\end{array}$ \\
\hline Kosten pro Teilnehmer (") & Beihilfeprogramm: 560 EUR (für die drei Unternehmerprojekte) \\
\hline Finanzierungsquellen & EU-Instrument für Heranführungshilfe; Kofinanzierung durch die nationale Regierung und lokale Organisationen. \\
\hline
\end{tabular}

(*) Beim Vergleich der Kosten pro Teilnehmer der verschiedenen Programme sollten die Dauer der Programme und die Unterschiede in den angebotenen Dienstleistungen beachtet werden. 


\section{Dänemark}

\begin{tabular}{|c|c|}
\hline \multicolumn{2}{|r|}{ Geschäftscoaching für ethnische Minderheiten } \\
\hline Zielgruppe & Unternehmer aus ethnischen Minderheiten \\
\hline Geleistete Unterstützung & Coaching, Weitervermittlungen \\
\hline Ziel & $\begin{array}{l}\text { Das Ziel des Programms besteht darin, die Rolle des Unternehmensberaters weg von der Familie und von Freunden hin zu } \\
\text { einem professionellen Betreuer zu verlagern, um für eine wirksamere Beratung zu sorgen. Dies dürfte die Erfolgsquote bei den } \\
\text { Unternehmern aus ethnischen Minderheiten in Vejle erhöhen. }\end{array}$ \\
\hline Status & 2004 gestartet; laufend. \\
\hline \multirow[t]{2}{*}{ Kurzprofil des Programms } & $\begin{array}{l}\text { Dieses Projekt baut durch die Bereitstellung professioneller Beratungsdienste persönliche Beziehungen mit Unternehmern in } \\
\text { Gemeinschaften von ethnischen Minderheiten und Zuwanderern auf. Der Betreuer hat die Funktion, professionelle Beratung } \\
\text { zu den Rechts- und Sozialnormen im neuen Land anzubieten und die sozialen Netzwerke der ethnischen Minderheiten } \\
\text { aufzubauen und zu stärken. Die Beratung erfolgt im Rahmen von informellen und flexiblen Abmachungen. Zunächst liegt das } \\
\text { Hauptaugenmerk auf dem Geschäftsplan: Die zentralen Elemente der Geschäftsidee sollen ermittelt und weiterentwickelt werden, } \\
\text { unter anderem die Möglichkeit, der Markt, der Finanzierungsbedarf und die Kompetenzen des Unternehmers. Auf der Grundlage } \\
\text { dieser Gespräche vermittelt der Betreuer die Teilnehmer an formelle Geschäfts- und Unternehmerschulungen weiter, die von den } \\
\text { Regionen und Kommunen angeboten werden. Im Anschluss an die Unternehmensgründung können die Teilnehmer weiterhin bei } \\
\text { ihrem Berater Coaching und Mentoring in Anspruch nehmen, um ihr Unternehmen weiterzuentwickeln und wachsen zu lassen. }\end{array}$ \\
\hline & $\begin{array}{l}\text { Anfangs waren die Empfänger dieser Unterstützung zum größten Teil türkischer Herkunft, inzwischen stammen die Teilnehmer } \\
\text { jedoch aus den unterschiedlichsten Gebieten, unter anderem aus Nordafrika, vom Horn von Afrika sowie aus Zentral- und } \\
\text { Osteuropa. }\end{array}$ \\
\hline Hintergrund & $\begin{array}{l}\text { Das Projekt wurde initiiert, weil allgemeine Dienstleistungen zur Unternehmensförderung nicht für Unternehmer aus } \\
\text { ethnischen Minderheiten und zugewanderte Unternehmer attraktiv waren. Eine der größten Herausforderungen für öffentliche } \\
\text { Unterstützungsprogramme ist es, eine Vertrauensbasis mit den Klienten aufzubauen. Ein Betreuer, der selbst aus einer } \\
\text { ethnischen Minderheit stammt, beweist gegenüber dem Klienten häufig ein besseres Einfühlungsvermögen und hat unter } \\
\text { Umständen ein tieferes Verständnis für die Herausforderungen, die sich dem Klienten stellen, und dessen Bedürfnisse } \\
\text { (insbesondere hinsichtlich nicht mit dem Unternehmen zusammenhängender Aspekten wie Einwanderung und Status der } \\
\text { Arbeitserlaubnis). }\end{array}$ \\
\hline Dauer der Unterstützung & $\begin{array}{l}\text { Die Teilnehmer müssen an einem zweistündigen Aufnahmegespräch teilnehmen. Anschließend werden die Dauer und Intensität } \\
\text { der Coaching-Beziehung zwischen dem Betreuer und dem Unternehmer verhandelt. }\end{array}$ \\
\hline Größte Herausforderungen & 1. Die Sicherung einer ausreichenden Finanzierung, um das Projekt aufrechtzuerhalten. \\
\hline \multirow[t]{2}{*}{ Auswirkungen } & Jährlich werden etwa 100 Unternehmer betreut, von denen $50 \%$ innerhalb eines Jahres ein Unternehmen gründen. \\
\hline & $\begin{array}{l}\text { Das Projekt wurde } 2007 \text { mit dem „European Trailblazer Award“ ausgezeichnet und } 2011 \text { im Interreg IVC-Projekt, Enspire EU } \\
\text { (Entrepreneurial inspiration for the European Union), als beste Vorgehensweise in Europa ausgewählt. }\end{array}$ \\
\hline Durchschnittliche jährliche Kosten & 20000 EUR \\
\hline Kosten pro Teilnehmer (") & 200 EUR \\
\hline Finanzierungsquellen & Europäischer Sozialfonds; Sachleistungen \\
\hline
\end{tabular}

(*) Beim Vergleich der Kosten pro Teilnehmer der verschiedenen Programme sollten die Dauer der Programme und die Unterschiede in den angebotenen Dienstleistungen beachtet werden. 


\section{Finnland}

\begin{tabular}{|c|c|}
\hline \multicolumn{2}{|r|}{ Beihilfe zur Unternehmensgründung } \\
\hline Zielgruppe & Arbeitslose und Menschen, die aus der abhängigen Erwerbstätigkeit in die selbstständige Erwerbstätigkeit wechseln. \\
\hline Geleistete Unterstützung & Überbrückungshilfe \\
\hline Ziel & $\begin{array}{l}\text { Das Ziel der „Beihilfe zur Unternehmensgründung“ ist die Stärkung neuer Unternehmensgründungen und die Förderung } \\
\text { der Beschäftigung unter den Arbeitslosen. Das Programm möchte einen Beitrag zur Erhöhung der Anzahl der neuen } \\
\text { Unternehmensgründungen in Finnland leisten, die Überlebensrate neuer gegründeter Unternehmen aufrechterhalten und } \\
\text { verbessern sowie Einzelne zur Unternehmensgründung animieren und ihr Wachstum und ihre Beschäftigung fördern. }\end{array}$ \\
\hline Status & 1984 gestartet; laufend. \\
\hline \multirow[t]{3}{*}{ Kurzprofil des Programms } & $\begin{array}{l}\text { Das Programm gewährt Zuwendungen, um neue Unternehmer in der Frühphase der Unternehmensentwicklung, wenn ein } \\
\text { Kundenstamm für das Unternehmen aufgebaut wird, zu unterstützen. Teilnehmen können Arbeitslose, die ein Unternehmen } \\
\text { gründen, sowie Menschen, die von einer abhängigen Erwerbstätigkeit in eine selbstständige Erwerbstätigkeit wechseln möchten. }\end{array}$ \\
\hline & $\begin{array}{l}\text { Die Zuwendung besteht aus einer Grundbeihilfe, um die Lebenshaltungskosten des Unternehmers für einen bestimmten } \\
\text { Zeitraum zu decken. Diese Grundbeihilfe belief sich im Jahr } 2014 \text { auf 32,66 EUR pro Tag. Sie wird in der Regel sechs Monate } \\
\text { lang geleistet, kann aber auf bis zu } 18 \text { Monate verlängert werden. Zusätzlich kann eine Ergänzungsbeihilfe gewährt werden, die } \\
\text { bis zu } 60 \% \text { der Höhe der Grundbeihilfe beträgt. Im Durchschnitt betrug die Beihilfe etwa } 800-850 \text { EUR monatlich für } 9,5 \text { Monate. }\end{array}$ \\
\hline & $\begin{array}{l}\text { Verwaltet wird die Beihilfe durch das TE-Büro (Büro für Beschäftigung und Wirtschaftsentwicklung), die Auswahl der } \\
\text { Beihilfeempfänger übernehmen jedoch externe Berater. }\end{array}$ \\
\hline Hintergrund & $\begin{array}{l}\text { Ursprünglich wurde die Beihilfe zur Bekämpfung einer schweren Rezession und hoher Arbeitslosenquoten eingerichtet. Sie } \\
\text { wurde außerdem als Möglichkeit betrachtet, das Wirtschaftswachstum, den Wirtschaftswandel und die Wirtschaftsentwicklung } \\
\text { zu fördern. }\end{array}$ \\
\hline Dauer der Unterstützung & Zuwendungen werden sechs Monate lang geleistet, können aber um weitere zwölf Monate verlängert werden. \\
\hline \multirow[t]{3}{*}{ Größte Herausforderungen } & $\begin{array}{l}\text { 1. Es ist schwierig, ein ausgeglichenes Verhältnis zu schaffen zwischen den sozialen Zielen der Unterstützung von Arbeitslosen } \\
\text { und der Notwendigkeit, für echte Erfolgschancen für die gegründeten Unternehmen zu sorgen. Zudem ist es für die Regierung } \\
\text { schwierig, denjenigen, die die Bewerbungen bewerten, Orientierungshilfen zu geben. }\end{array}$ \\
\hline & $\begin{array}{l}\text { 2. Die Beihilfeempfänger sind häufig nicht mit der Höhe und der Dauer der Beihilfe zufrieden. Dies deutet darauf hin, dass die } \\
\text { Regierung die Beihilfeempfänger möglicherweise besser darüber informieren sollte, dass es sich bei der Beihilfe nicht um eine } \\
\text { Start-up-Finanzierung handelt. }\end{array}$ \\
\hline & $\begin{array}{l}\text { 3. Den Beihilfeempfängern sind häufig keine anderen Finanzierungsmöglichkeiten bekannt, auf die sie in Bezug auf eine Start-up- } \\
\text { Finanzierung zurückgreifen können. }\end{array}$ \\
\hline \multirow[t]{2}{*}{ Auswirkungen } & $\begin{array}{l}\text { Rund } 50 \% \text { der Beihilfeempfänger gaben an, die Beihilfe sei notwendig gewesen, um ihr Unternehmen zu gründen. } 38 \% \text { der im } \\
\text { Jahr } 2005 \text { gegründeten Unternehmen waren } 2012 \text { immer noch in Betrieb (sowie } 41 \% \text { aus dem Jahr } 2006 \text { und } 61 \% \text { aus dem } \\
\text { Jahr 2007). }\end{array}$ \\
\hline & 50-80 \% der Unternehmer arbeiteten in Vollzeit für ihr Start-up (abhängig vom Jahr) und $66 \%$ von innen stellten Mitarbeiter ein. \\
\hline Durchschnittliche jährliche Kosten & 37,5 Millionen EUR \\
\hline Kosten pro Teilnehmer (“) & 7650 EUR (nur für die Beihilfe, ohne Berücksichtigung der Betriebskosten) \\
\hline Finanzierungsquellen & Ministerium für Beschäftigung und Wirtschaft (95\%); Europäischer Sozialfonds (5\%) \\
\hline
\end{tabular}

$\left(^{*}\right)$ Beim Vergleich der Kosten pro Teilnehmer der verschiedenen Programme sollten die Dauer der Programme und die Unterschiede in den angebotenen Dienstleistungen beachtet werden. 


\section{Frankreich}

\begin{tabular}{|c|c|}
\hline \multicolumn{2}{|r|}{ Adie Mikrofranchising } \\
\hline Zielgruppe & In erster Linie Arbeitslose, aber alle sozial ausgegrenzten Gruppen kommen infrage \\
\hline Geleistete Unterstützung & Ein Geschäftsmodell (d. h. Mikrofranchising) und integrierte Unterstützungsdienstleistungen \\
\hline Ziel & $\begin{array}{l}\text { Das Ziel besteht darin, den Eintritt in die selbstständige Erwerbstätigkeit zu erleichtern, indem Klienten ein risikoarmes, sofort } \\
\text { einsatzbereites Geschäftsmodell geboten wird, das ihnen eine selbstständige Erwerbstätigkeit ermöglicht. }\end{array}$ \\
\hline Status & 2011 gestartet; laufend. \\
\hline \multirow[t]{3}{*}{ Kurzprofil des Programms } & $\begin{array}{l}\text { Die Klienten von Adie sind in der Regel Arbeitslose und Sozialhilfeempfänger. Das Mikrofranchising-Programm bietet ein } \\
\text { sofort einsatzbereites Geschäftsmodell sowie verschiedene Unterstützungsleistungen für die Unternehmensgründung (z. B. } \\
\text { Schulungen, Unternehmensberatung), um zum Erfolg des Mikrofranchising beizutragen. }\end{array}$ \\
\hline & $\begin{array}{l}\text { Adie ermittelt potenzielle Mikrofranchisegeber über seine Unternehmensnetzwerke. Aktuell gibt es zwei Arten von } \\
\text { Mikrofranchise-Netzwerken: solche, die in Zusammenarbeit mit Großkonzernen entwickelt werden, und solche, die in } \\
\text { Zusammenarbeit mit den Unternehmern entwickelt werden. Der einzige Unterschied zwischen den beiden Modellen ist die } \\
\text { potenzielle Größe des Netzwerks. }\end{array}$ \\
\hline & $\begin{array}{l}\text { Als Mikrofranchisenehmer infrage kommende Kandidaten werden befragt und hinsichtlich ihrer Motivation und ihres } \\
\text { betriebswirtschaftlichen Potenzials beurteilt. Adie begründet ein Vertragsverhältnis zwischen allen Parteien. Der } \\
\text { Mikrofranchisenehmer muss dem Mikrofranchisegeber für die Nutzung des Geschäftsmodells Lizenzgebühren zahlen. Unter } \\
\text { Umständen unterstützt Adie den Mikrofranchisenehmer auch mit Zugang zu Finanzierung und Schulungen. }\end{array}$ \\
\hline Hintergrund & $\begin{array}{l}\text { Adie rief seine Mikrofranchising-Initiative ins Leben, weil immer mehr Menschen in Zweigstellen der Vereinigung kamen, die } \\
\text { bereit waren, ein eigenes Unternehmen zu gründen, aber keine tragfähige Geschäftsidee hatten. Ein traditionelles Franchising } \\
\text { ist für Unternehmer mit niedrigem Einkommen nicht erschwinglich, und es gab keine sonstige Unterstützung für diese } \\
\text { Unternehmer, die ihr Unternehmen nicht allein gründen möchten. }\end{array}$ \\
\hline Dauer der Unterstützung & Die Dauer der Unterstützung hängt von den Bedürfnissen des Klienten ab. \\
\hline \multirow[t]{3}{*}{ Größte Herausforderungen } & $\begin{array}{l}\text { 1. Berufliche Zertifizierungsanforderungen stellen eine Herausforderung dar, da viele potenzielle Mikrofranchising-Unternehmen } \\
\text { in Sektoren tätig sind, in denen Mitarbeiter Schulungen und Zertifikate vorweisen müssen. Die Einholung dieser Zertifikate ist für } \\
\text { die Klienten von Adie häufig schwierig. }\end{array}$ \\
\hline & $\begin{array}{l}\text { 2. Die bisherigen Erfahrungen ergaben keine konsistenten Erfolgsprädiktoren, die man für das Auswahlverfahren für die } \\
\text { Kandidaten heranziehen könnte. }\end{array}$ \\
\hline & 3. Für eine Ausweitung des Programms werden mehr Freiwillige und mehr Schulungen für Freiwillige benötigt. \\
\hline Auswirkungen & $\begin{array}{l}\text { Drei Mikrofranchising-Netzwerke wurden ins Leben gerufen, die mehr als } 150 \text { Möglichkeiten für eine selbstständige } \\
\text { Erwerbstätigkeit geschaffen haben. Für fünf Netzwerke werden derzeit Pilotprojekte durchgeführt. }\end{array}$ \\
\hline Durchschnittliche jährliche Kosten & k. A. \\
\hline Kosten pro Teilnehmer (") & 8000 EUR \\
\hline Finanzierungsquellen & Adie \\
\hline
\end{tabular}

(*) Beim Vergleich der Kosten pro Teilnehmer der verschiedenen Programme sollten die Dauer der Programme und die Unterschiede in den angebotenen Dienstleistungen beachtet werden. 


\section{Deutschland}

\begin{tabular}{|c|c|}
\hline \multicolumn{2}{|r|}{ Gründungszuschuss } \\
\hline Zielgruppe & Arbeitslose \\
\hline Geleistete Unterstützung & Überbrückungshilfe \\
\hline Ziel & $\begin{array}{l}\text { Das Ziel des Überbrückungsgelds besteht darin, Arbeitslose bei der Gründung eines Unternehmens finanziell zu unterstützen. } \\
\text { Dieses Programm soll Arbeitslosen eine Alternative zu den typischen Strategien der Arbeitssuche bieten, um der Arbeitslosigkeit } \\
\text { zu entkommen. }\end{array}$ \\
\hline \multirow[t]{2}{*}{ Status } & Gründungszuschuss: 2006-2011 (abgeschlossen) \\
\hline & Neuer Gründungszuschuss: 2012 bis heute (laufend) \\
\hline Kurzprofil des Programms & $\begin{array}{l}\text { Arbeitslosen werden Zuschüsse gewährt, um ihnen die Gründung eines eigenen Unternehmens zu erleichtern. Die betroffene } \\
\text { Person erhält denselben Betrag, den sie im Rahmen der Arbeitslosenunterstützung erhalten hätte, zuzüglich } 300 \text { EUR für } \\
\text { Sozialversicherungsbeiträge. Die Leistungen werden für sechs Monate gewährt. Es besteht die Möglichkeit, sie für weitere } \\
\text { neun Monate zu verlängern (vorausgesetzt, die Person führt ihre selbstständige Erwerbstätigkeit fort). Um diese Zuwendung } \\
\text { zu erhalten, muss die Person noch einen Anspruch auf Arbeitslosenunterstützung von mindestens } 150 \text { Tagen haben und } \\
\text { einen Geschäftsplan vorlegen, der durch die Handelskammer oder eine ähnliche Einrichtung genehmigt wurde. Die Gestaltung } \\
\text { des Programms soll Arbeitslose in die Lage versetzen, sich auf die Gründung eines eigenen Unternehmens zu konzentrieren, } \\
\text { während ihre Lebenshaltungskosten durch den Zuschuss gedeckt werden. }\end{array}$ \\
\hline Hintergrund & $\begin{array}{l}\text { Diesem Zuschuss liegt der Umstand zugrunde, dass für Arbeitslose eine Startkapitallücke existiert und sie verpflichtet } \\
\text { sind, auf ihre Arbeitslosenunterstützung zu verzichten, wenn sie sich auf eine Gründung vorbereiten. Das wichtigste Ziel } \\
\text { dieses Finanzierungsinstruments besteht darin, die Lebenshaltungs- und Sozialversicherungskosten zu decken. So soll } \\
\text { Existenzgründern, die aus der Arbeitslosigkeit kommen, dabei geholfen werden, die erste Phase der selbstständigen } \\
\text { Erwerbstätigkeit zu überstehen, in der Unternehmen sehr anfällig sind und eventuell noch keine adäquaten Einnahmen abwerfen. }\end{array}$ \\
\hline Dauer der Unterstützung & Zuwendungen werden sechs Monate lang gezahlt, können aber um weitere neun Monate verlängert werden. \\
\hline \multirow[t]{3}{*}{ Größte Herausforderungen } & $\begin{array}{l}\text { 1. Die Minimierung von Verlusten wegen Mitnahmeeffekten, da einige Personen, die unabhängig von dem Zuschuss } \\
\text { selbstständig erwerbstätig geworden wären, dennoch finanziert werden. }\end{array}$ \\
\hline & $\begin{array}{l}\text { 2. Die Minimierung von Verdrängungseffekten für bestehende Unternehmen, die mit aus öffentlicher Hand bezuschussten } \\
\text { Unternehmen konkurrieren müssen. }\end{array}$ \\
\hline & $\begin{array}{l}\text { 3. Die Verhinderung der „Scheinselbstständigkeit“, bei der Arbeitnehmer durch Selbstständige ersetzt werden, die effektiv wie } \\
\text { reguläre Arbeitnehmer, nur zu günstigeren Kosten tätig sind. }\end{array}$ \\
\hline \multirow[t]{3}{*}{ Auswirkungen } & $\begin{array}{l}\text { Die Überlebensraten für Start-ups lagen } 56 \text { Monate nach der Gründung zwischen } 55 \% \text { und } 70 \% \text { (abhängig von Geschlecht, } \\
\text { Region und anderen Faktoren). Nach fünf Jahren hatte der allgemeine Durchschnitt eine } 50 \% \text { ige Überlebenschance. } 40 \% \text { der } \\
\text { Bezieher hatten mindestens einen weiteren Mitarbeiter in ihrem Unternehmen. Die Arbeitsplatzschaffungsquote lag für jeden } \\
\text { Zuschuss bei 1:1. }\end{array}$ \\
\hline & Zwischen $20 \%$ und $23 \%$ der Bezieher fanden im Anschluss an das Programm eine regelmäßige abhängige Erwerbstätigkeit. \\
\hline & $\begin{array}{l}\text { Die Programmteilnehmer waren } 20 \text { Monate länger beschäftigt und hatten im Durchschnitt ein höheres Arbeitseinkommen als } \\
\text { Arbeitslose, die ihr Unternehmen ohne jegliche Unterstützung gründeten. }\end{array}$ \\
\hline Durchschnittliche jährliche Kosten & Zwischen 1,5 Milliarden EUR und 3,5 Milliarden EUR, abhängig vom Jahr und Programm \\
\hline Kosten pro Teilnehmer (") & 11000-13000 EUR \\
\hline Finanzierungsquellen & Nationalregierung (Gesetzliche Arbeitslosenversicherung) \\
\hline
\end{tabular}

(*) Beim Vergleich der Kosten pro Teilnehmer der verschiedenen Programme sollten die Dauer der Programme und die Unterschiede in den angebotenen Dienstleistungen beachtet werden. 


\section{Ungarn}

\begin{tabular}{|c|c|}
\hline \multicolumn{2}{|r|}{ Kiutprogram } \\
\hline Zielgruppe & Arbeitslose in benachteiligten Gebieten (in der Praxis zum größten Teil Roma) \\
\hline Geleistete Unterstützung & Finanzierung (Darlehen), Coaching und Mentoring, Schulungen, Unternehmensberatung \\
\hline Ziel & $\begin{array}{l}\text { Das Programm strebte die Förderung der sozialen Mobilität und der Eingliederung benachteiligter Menschen in benachteiligten } \\
\text { Gebieten an, indem diesen durch die Gründung und Eintragung eines Kleinunternehmens die Aufnahme einer selbstständigen } \\
\text { Erwerbstätigkeit ermöglicht wurde. }\end{array}$ \\
\hline Status & 2010-2012; abgeschlossen. \\
\hline \multirow[t]{3}{*}{ Kurzprofil des Programms } & $\begin{array}{l}\text { Dieses Gruppenkreditvergabeprogramm lief von Oktober } 2010 \text { bis Juni } 2012 \text { und basierte auf dem Grameen-Modell. } \\
\text { Außendienstmitarbeiter besuchten infrage kommende Gebiete, um für das Programm zu werben, und kehrten dann nochmals } \\
\text { dorthin zurück, um sich mit Interessierten zu Aufnahmegesprächen zu treffen. Die ausgewählten Kandidaten wurden } \\
\text { in Kreditgruppen aufgeteilt, und die Gruppenmitglieder erhielten der Reihe nach ihre Kredite. Es mussten wöchentliche } \\
\text { Rückzahlungen erfolgen. Säumnisse hatten zur Folge, dass andere Gruppenmitglieder ihre Kredite nicht erhielten. In jeder } \\
\text { Gruppe war ein Leiter dafür zuständig, sicherzustellen, dass die Mitglieder die Kreditbedingungen erfüllten. Die Gruppenleiter } \\
\text { erhielten ihren Kredit als Letztes. }\end{array}$ \\
\hline & $\begin{array}{l}\text { Bei der Umsetzung des Projekts und der individuellen Unterstützung der Klienten spielten Außendienstmitarbeiter eine wichtige } \\
\text { Rolle. Die Unterstützung beinhaltete Hilfestellung bei der Einholung der notwendigen Gewerbeerlaubnis und der Eröffnung von } \\
\text { Bankkonten sowie Coaching und betriebswirtschaftliche Schulungen. }\end{array}$ \\
\hline & $\begin{array}{l}\text { Aufgrund der schleppenden Inanspruchnahme und dem schlechten Abschneiden der ersten Klientenkohorte wurde das } \\
\text { Aufnahmeverfahren nach dem ersten Jahr überarbeitet. }\end{array}$ \\
\hline Hintergrund & $\begin{array}{l}\text { Die Zielgruppe von Kiútprogram waren Menschen in den am stärksten benachteiligten Gebieten Ungarns - hauptsächlich, aber } \\
\text { nicht ausschließlich, Roma. Das Ziel des Programms war es, eine Lücke im Sozialleistungssystem zu schließen. Dafür wurden } \\
\text { finanzielle Unterstützung, Schulungen und persönliches Coaching bereitgestellt, die Menschen bei der Unternehmensgründung } \\
\text { helfen sollten, damit sie auf dem Arbeitsmarkt und in der Gesellschaft aktiv bleiben können. }\end{array}$ \\
\hline Dauer der Unterstützung & 18 Monate \\
\hline \multirow[t]{3}{*}{ Größte Herausforderungen } & $\begin{array}{l}\text { 1. Die Einhaltung der Ziele und Zielvorgaben. Das Programm änderte seinen Zielmarkt während der dreijährigen Laufzeit } \\
\text { erheblich. }\end{array}$ \\
\hline & $\begin{array}{l}\text { 2. Die Auswahl der Teilnehmer stellte eine Herausforderung dar. Es war schwierig, potenzielle Unternehmer zu identifizieren, die } \\
\text { alle Auswahlkriterien erfüllten. Im Ergebnis hatten viele Teilnehmer keinen Erfolg. }\end{array}$ \\
\hline & $\begin{array}{l}\text { 3. Die Teilnehmer lebten in benachteiligten Gebieten und waren somit mit der Herausforderung relativ schwacher } \\
\text { regionaler Märkte und geschäftlicher Netzwerke konfrontiert. Sie stützten sich in hohem Maße auf die Unterstützung des } \\
\text { Außendienstmitarbeiters. }\end{array}$ \\
\hline \multirow[t]{3}{*}{ Auswirkungen } & $\begin{array}{l}192 \text { Menschen aus benachteiligten Gruppen bewarben sich. Von diesen wurden } 138 \text { für die Teilnahme an dem Programm } \\
\text { ausgewählt. }\end{array}$ \\
\hline & $\begin{array}{l}95 \% \text { der Klienten behaupteten, dass das Programm die Hauptfinanzierungsquelle für die Gründung ihres eigenen Unternehmens } \\
\text { darstellte. }\end{array}$ \\
\hline & $45 \%$ der Unternehmen waren ein Jahr, nachdem die Teilnehmer das Programm abgeschlossen hatten, immer noch in Betrieb. \\
\hline Durchschnittliche jährliche Kosten & 466667 EUR \\
\hline Kosten pro Teilnehmer (") & 10144 EUR \\
\hline Finanzierungsquellen & Europäische Union (85\%); nationale Regierung (15\%) \\
\hline
\end{tabular}

$\left(^{*}\right)$ Beim Vergleich der Kosten pro Teilnehmer der verschiedenen Programme sollten die Dauer der Programme und die Unterschiede in den angebotenen Dienstleistungen beachtet werden. 


\section{Irland}

\begin{tabular}{|c|c|}
\hline \multicolumn{2}{|r|}{ Going for Growth } \\
\hline Zielgruppe & Unternehmerinnen mit Wachstumsambitionen \\
\hline Geleistete Unterstützung & Gruppen- und Peer-basiertes Coaching und Mentoring, Rollenvorbilder \\
\hline Ziel & $\begin{array}{l}\text { Das Ziel ist die Unterstützung ehrgeiziger Unternehmerinnen, die für ihr Unternehmen ein Wachstum anstreben und ihre } \\
\text { Netzwerke ausweiten möchten. }\end{array}$ \\
\hline Status & 2009 gestartet; laufend. \\
\hline \multirow[t]{2}{*}{ Kurzprofil des Programms } & $\begin{array}{l}\text { Going for Growth fördert Unternehmerinnen mit Wachstumsbestrebungen. Durch die Konzentration auf Wachstumsziele und } \\
\text { zeitlich definierte Meilensteine soll die Unternehmensentwicklung unterstützt werden. Die Initiative nutzt freiwillige Beiträge von } \\
\text { erfolgreichen Unternehmern, die in sechsmonatigen Zyklen Sitzungen für Gruppen- und Peer-basiertes Coaching und Mentoring } \\
\text { leiten. Diese Gruppentreffen werden durch freiwillige „Leiterinnen“ moderiert, die erfolgreiche Unternehmerinnen sind. Die } \\
\text { Leiterinnen teilen ihre Erfahrungen mit der Gruppe, pflegen eine Kultur des Vertrauens und der Zusammenarbeit und fördern } \\
\text { den Austausch von Erfahrungen und Herausforderungen. Das Gruppen-Mentoring bietet Unterstützung, schafft einen positiven } \\
\text { Gruppenzwang und animiert die Unternehmerinnen dazu, ihre Ziele zu erreichen. Zu dem Projekt gehört außerdem ein nationales } \\
\text { Forum, das alle Leiterinnen und Teilnehmer zu Entwicklungs-Workshops und zum Zweck der Ausweitung ihrer Netzwerke } \\
\text { zusammenbringt. }\end{array}$ \\
\hline & $\begin{array}{l}\text { Going for Growth setzt sich zudem für eine positive Darstellung von Unternehmerinnen in den Medien ein, um eine } \\
\text { Wahrnehmung der Frauen als wachstumsfähig zu fördern. }\end{array}$ \\
\hline Hintergrund & $\begin{array}{l}\text { In Irland sind Frauen seltener als Männer unternehmerisch tätig. Frauen sind im Unternehmertum in der Tendenz weniger } \\
\text { selbstbewusst, nehmen unternehmerische Möglichkeiten seltener war und haben weniger entwickelte Netzwerke als Männer. } \\
\text { Entsprechend hegt ein kleinerer Anteil der Unternehmerinnen erhebliche Wachstumsbestrebungen. }\end{array}$ \\
\hline Dauer der Unterstützung & Jeder Zyklus dauert sechs Monate. \\
\hline \multirow[t]{3}{*}{ Größte Herausforderungen } & $\begin{array}{l}\text { 1. Die Mittel für das Projekt stammen in erster Linie aus der öffentlichen Hand und sind daher häufig kurzfristiger Natur. Die } \\
\text { Ausweitung des Projekts mit einem relativ festen Haushalt stellt sowohl hinsichtlich seiner Größenordnung als auch seines } \\
\text { Umfangs eine Herausforderung dar. Eine wachsende Anzahl privater Sponsoren ermöglicht die Entwicklung neuer Elemente und } \\
\text { ergänzender Initiativen. }\end{array}$ \\
\hline & $\begin{array}{l}\text { 2. Teilnehmerinnen anzuziehen war in den Anfangsjahren aufgrund der Zahl der bestehenden Netzwerke und Programme } \\
\text { für Unternehmerinnen eine Herausforderung. Nun besteht die Schwierigkeit, dass die Nachfrage nach Programmplätzen die } \\
\text { Kapazität übersteigt. }\end{array}$ \\
\hline & 3. Es ist eine Herausforderung, Unternehmerinnen als Leiterinnen zu gewinnen, die dem Projekt freiwillig ihre Zeit widmen. \\
\hline Auswirkungen & $\begin{array}{l}\text { Das Projekt befindet sich derzeit in seinem } 7 \text {. Zyklus, und mehr als } 400 \text { Teilnehmerinnen haben es bislang in Anspruch } \\
\text { genommen. Mehr als } 70 \% \text { der Teilnehmerinnen, die den } 6 \text {. Zyklus abgeschlossen haben, gaben an, während des } \\
\text { sechsmonatigen Zeitraums einen Umsatzanstieg verzeichnet zu haben. Durchschnittlich lag das Wachstum bei } 15 \% \text {. Während } \\
\text { dieses Zeitraums stellten die Teilnehmerinnen } 88 \text { Menschen zusätzlich ein und beschäftigten zehn Praktikanten. Die Zahl der } \\
\text { Exporteure unter den Teilnehmerinnen stieg um } 22 \% \text {. }\end{array}$ \\
\hline Durchschnittliche jährliche Kosten & k. A. \\
\hline Kosten pro Teilnehmer (*) & 2000 EUR pro Zyklus \\
\hline \multirow[t]{3}{*}{ Finanzierungsquellen } & $\begin{array}{l}\text { Die Maßnahme „Equality for Women“ („Gleichstellung für Frauen“), die über das operationelle Programm für Investitionen in das } \\
\text { Humankapitel durch den Europäischen Sozialfonds finanziert wird; }\end{array}$ \\
\hline & $\begin{array}{l}\text { Enterprise Ireland, die für die Entwicklung und das Wachstum irischer Unternehmen verantwortliche Regierungsorganisation; } \\
\text { das Ministerium für Justiz und Gleichstellung über den Zeitraum von } 2010 \text { bis einschließlich } 2014 .\end{array}$ \\
\hline & Im Jahr 2015 wurde auch KPMG ein Sponsor. \\
\hline
\end{tabular}

$\left(^{*}\right)$ Beim Vergleich der Kosten pro Teilnehmer der verschiedenen Programme sollten die Dauer der Programme und die Unterschiede in den angebotenen Dienstleistungen beachtet werden. 


\section{Italien}

Invitalias Unterstützung für das Unternehmertum Arbeitsloser

\begin{tabular}{|c|c|}
\hline Zielgruppe & Arbeitslose, darunter vor allem arbeitslose junge Menschen (18- bis 35-Jährige) sowie Arbeitslose aus den Südregionen Italiens \\
\hline Geleistete Unterstützung & Finanzierung (Beihilfe und zinsverbilligte Darlehen), Schulungen und Unternehmensberatung \\
\hline Ziel & $\begin{array}{l}\text { Die Ziele von Invitalias Programm für das Unternehmertum Arbeitsloser konzentrieren sich auf Unternehmensgründungen und } \\
\text { sollen sicherstellen, dass Arbeitslose die Möglichkeit erhalten, in das Unternehmertum einzusteigen. }\end{array}$ \\
\hline Status & 1996 gestartet; laufend. \\
\hline Kurzprofil des Programms & $\begin{array}{l}\text { Dieses nationale Programm besteht aus vier gesonderten Phasen. Phase } 1 \text { besteht aus Werbemaßnahmen für das Programm } \\
\text { mit Seminaren zur Kontaktaufnahme und Workshops für Bewerber. Phase } 2 \text { ist das Aufnahmeverfahren. Die Bewerber sind } \\
\text { verpflichtet, einen Geschäftsplan zu erstellen, der von Invitalia bewertet wird. Lokale Partner (z. B. Iokale Entwicklungsagenturen) } \\
\text { beurteilen die Eignung der Bewerbung und des Geschäftsplans und treffen die Kandidaten. Genehmigte Vorschläge kommen } \\
\text { in Phase 3, die in Form von drei verschiedenen Unterstützungssparten durchgeführt wird. Die Teilnehmer werden einem der } \\
\text { folgenden Programme zugeteilt: selbstständige Erwerbstätigkeit, Mikrounternehmen und Franchising. Im Rahmen jeder dieser } \\
\text { Maßnahmen können die Teilnehmer eine Startbeihilfe von bis zu } 5165 \text { EUR, ein zinsverbilligtes Darlehen mit einer Laufzeit } \\
\text { von bis zu sieben Jahren und Hilfe bei der Geschäftsplanung, Schulungen, Mentoring und Unternehmensberatung erhalten. } \\
\text { Das Unterstützungsniveau ist je nach Programm unterschiedlich. Rund } 85 \% \text { der Bewerber wählen die Sparte „selbstständige } \\
\text { Erwerbstätigkeit“, } 14 \% \text { „Mikrounternehmen“ und weniger als } 1 \% \text { „Franchising“. Schließlich wertet Invitalia in Phase } 4 \text { die } \\
\text { Auswirkungen des Programms durch dauerhafte Überwachung der finanzierten Unternehmen und Datenerhebung von diesen } \\
\text { Unternehmen aus. }\end{array}$ \\
\hline Hintergrund & $\begin{array}{l}\text { Es spricht im Allgemeinen vieles für die Förderung von Unternehmensgründungen durch Arbeitslose. Auch wenn die } \\
\text { Erkenntnisse in der Europäischen Union gemischt sind, führte die wachsende Arbeitslosigkeit in Italien dazu, dass neue } \\
\text { Möglichkeiten zur Schaffung von Arbeitsplätzen gefunden und Methoden erforscht werden müssen, um Arbeitslose wirksam } \\
\text { wieder auf den Arbeitsmarkt zu bringen. }\end{array}$ \\
\hline Dauer der Unterstützung & $\begin{array}{l}\text { Unterstützung in Form von Unternehmensberatung im ersten Betriebsjahr. Finanzierungsmaßnahmen können bis zu sieben Jahre } \\
\text { dauern. }\end{array}$ \\
\hline \multirow[t]{3}{*}{ Größte Herausforderungen } & $\begin{array}{l}\text { 1. Die Verwaltung von Screening- und Aufnahmeverfahren mit begrenzten Ressourcen für Programmleiter und ein hohes } \\
\text { Bewerberaufkommen (bis zu } 10000 \text { pro Jahr). }\end{array}$ \\
\hline & $\begin{array}{l}\text { 2. Die Bereitstellung ausreichender, hochwertiger individueller Unterstützung für die Teilnehmer, um ihre Erfolgschancen zu } \\
\text { erhöhen. }\end{array}$ \\
\hline & 3. Die Entwicklung adäquater Partnerschaften, die das Programm fördern und Bestandteile der Dienstleistungen erbringen. \\
\hline Auswirkungen & $\begin{array}{l}\text { Zwischen } 1996 \text { und } 2013 \text { gingen } 327280 \text { Bewerbungen ein. Daraus wurden } 111124 \text { neue Unternehmen gegründet. Damit } \\
\text { wurden } 193084 \text { zusätzliche Arbeitsplätze geschaffen. Die Investitionen in diese neuen Unternehmen beliefen sich auf } \\
\text { 4032,7 Millionen EUR. }\end{array}$ \\
\hline Durchschnittliche jährliche Kosten & 120 Millionen EUR \\
\hline Kosten pro Teilnehmer (“) & Rund 36000 EUR pro geschaffenem Unternehmen (einschließlich finanzieller Beihilfe). \\
\hline Finanzierungsquellen & Europäischer Fonds für regionale Entwicklung; nationale Regierung \\
\hline
\end{tabular}

(*) Beim Vergleich der Kosten pro Teilnehmer der verschiedenen Programme sollten die Dauer der Programme und die Unterschiede in den angebotenen Dienstleistungen beachtet werden. 


\section{Lettland}

\begin{tabular}{|c|c|}
\hline & Maßnahme zur Aufnahme einer gewerblichen Tätigkeit oder selbstständigen Erwerbstätigkeit \\
\hline Zielgruppe & Arbeitslose \\
\hline Geleistete Unterstützung & Finanzierung (Beihilfe, monatliche Zuwendungen), Unternehmensberatung \\
\hline Ziel & $\begin{array}{l}\text { Hilfe für Arbeitslose, durch eine selbstständige Erwerbstätigkeit auf den Arbeitsmarkt zurückzukehren und ihr Start-up für } \\
\text { mindestens zwei Jahre aufrechtzuerhalten. }\end{array}$ \\
\hline Status & 2008 gestartet; laufend. \\
\hline \multirow[t]{4}{*}{ Kurzprofil des Programms } & $\begin{array}{l}\text { Die Unterstützung wird in dieser Maßnahme in zwei Phasen geleistet. Zunächst nehmen die Teilnehmer an einer Reihe von } \\
\text { Beratungsterminen teil, bei denen sie individuelle Unterstützung bei der Vorbereitung und Entwicklung eines Geschäftsplans } \\
\text { erhalten. Jeder Teilnehmer kann innerhalb von sechs Wochen an } 20 \text { individuellen Beratungssitzungen teilnehmen. Die Beratung } \\
\text { erfolgt durch Geschäftsexperten, die von der staatlichen Arbeitsagentur (Nodarbinātības valsts aǵentūra, NVA) beauftragt } \\
\text { werden. Das Hauptaugenmerk dieser Sitzungen liegt auf der Ermittlung von Geschäftsideen und dem Entwurf eines Plans auf } \\
\text { dieser Grundlage. }\end{array}$ \\
\hline & $\begin{array}{l}\text { Sobald die Beratung abgeschlossen ist und die Teilnehmer einen Geschäftsplan haben, führt die NVA ein Evaluierungsverfahren } \\
\text { durch, um die Projekte auszuwählen, die in der zweiten Unterstützungsphase gefördert werden. Die zweite Unterstützungsphase } \\
\text { besteht aus zusätzlichen individuellen Unternehmensberatungsterminen und finanziellen Beihilfen. }\end{array}$ \\
\hline & $\begin{array}{l}\text { Das Evaluierungsverfahren konzentriert sich auf die Qualität und Realisierbarkeit der Geschäftspläne, die in der ersten } \\
\text { Unterstützungsphase entwickelt wurden. Die NVA stellt einen Evaluierungsausschuss zusammen, der aus Branchenexperten und } \\
\text { Experten im Bereich Unternehmensgründung und -entwicklung besteht. Der Ausschuss prüft das Potenzial der Geschäftspläne } \\
\text { unter Berücksichtigung der darin enthaltenen Produkt- bzw. Dienstleistungsinnovationen, der Einzigartigkeit des Produkts bzw. } \\
\text { der Dienstleistung und der potenziellen Nachfrage nach dem Produkt bzw. der Dienstleistung. }\end{array}$ \\
\hline & $\begin{array}{l}\text { Die ausgewählten Projekte kommen für Folgendes infrage: Unternehmensberatung im ersten Jahr der Umsetzung der } \\
\text { selbstständigen Erwerbstätigkeit oder der Unternehmensgründung ( } 20 \text { Beratungstermine); eine Beihilfe für die Umsetzung eines } \\
\text { Geschäftsplans in Höhe von höchstens } 2845,74 \text { EUR gemäß der genehmigten Schätzung; eine monatliche Beihilfe in der frühen } \\
\text { Umsetzungsphase (den ersten sechs Monaten) in Höhe des staatlich festgelegten Mindestlohns (320 EUR). }\end{array}$ \\
\hline Hintergrund & $\begin{array}{l}\text { Nach der Wirtschaftskrise erhöhte sich die Arbeitslosigkeit in Lettland in schnellerem Maße als in der Europäischen Union } \\
\text { insgesamt. Dies belastete die öffentlichen Arbeitsverwaltungen. Dieses Pilotprojekt wurde gestartet, um das Potenzial einer } \\
\text { Unterstützung von Arbeitslosen bei der selbstständigen Erwerbstätigkeit zu testen. }\end{array}$ \\
\hline Dauer der Unterstützung & $\begin{array}{l}\text { Die Unterstützung vor der Unternehmensgründung besteht aus individuellen Beratungsterminen über einen Zeitraum von sechs } \\
\text { Wochen. Die Unterstützung nach der Gründung besteht aus einer Beihilfe für sechs Monate. }\end{array}$ \\
\hline \multirow[t]{3}{*}{ Größte Herausforderungen } & $\begin{array}{l}\text { 1. Die Auswahl der Teilnehmer stellt eine Herausforderung dar. Es besteht die Notwendigkeit, Ergebnisse zu erzielen, was Anlass } \\
\text { zu strengen Auswahlkriterien für die Teilnehmer gab. Dies hat jedoch die Anzahl der Arbeitslosen, die für das Programm infrage } \\
\text { kommen, beschränkt. }\end{array}$ \\
\hline & $\begin{array}{l}\text { 2. Die Sicherung der Finanzierung ist schwierig. Da es sich um ein kleines Pilotprojekt handelt, ist die Finanzierung ständig } \\
\text { gefährdet. }\end{array}$ \\
\hline & $\begin{array}{l}\text { 3. Arbeitslose davon zu überzeugen, zu einer formellen Tätigkeit zurückzukehren, da Tätigkeiten in der informellen Wirtschaft } \\
\text { weit verbreitet sind. }\end{array}$ \\
\hline Auswirkungen & $\begin{array}{l}\text { Zwischen } 2008 \text { und } 2014 \text { haben insgesamt } 1228 \text { Arbeitslose an den anfänglichen Beratungen zur Erstellung eines } \\
\text { Geschäftsplans teilgenommen. Ende } 2014 \text { hatten } 267 \text { Unternehmer das Zwei-Jahres-Programm abgeschlossen. Derzeit erhalten } \\
129 \text { Arbeitslose Unterstützung durch dieses Programm. }\end{array}$ \\
\hline Durchschnittliche jährliche Kosten & 288300 EUR \\
\hline Kosten pro Teilnehmer (") & 5628,83 EUR (Höchstbetrag einschließlich Beihilfe und Zuwendungen.) \\
\hline Finanzierungsquellen & Nationale Regierung \\
\hline
\end{tabular}

(*) Beim Vergleich der Kosten pro Teilnehmer der verschiedenen Programme sollten die Dauer der Programme und die Unterschiede in den angebotenen Dienstleistungen beachtet werden. 


\section{Litauen}

\begin{tabular}{|c|c|}
\hline \multicolumn{2}{|r|}{ Förderungsfonds für das Unternehmertum } \\
\hline Zielgruppe & $\begin{array}{l}\text { Landesweites Programm, doch Arbeitslosen, jungen Menschen (unter } 29 \text { Jahre), Älteren (über } 50 \text { Jahre) und Menschen mit } \\
\text { Behinderungen wird vorrangiger Zugang gewährt }\end{array}$ \\
\hline Geleistete Unterstützung & Mikrokredite, Darlehensgarantien, Schulungen, Unternehmensberatung \\
\hline Ziel & $\begin{array}{l}\text { Das Ziel des Förderungsfonds für das Unternehmertum (Entrepreneurship Promotion Fund, EPF) ist es, die Zahl der } \\
\text { Unternehmensgründungen und der selbstständig Erwerbstätigen zu erhöhen und prioritäre Gruppen aus im Unternehmertum } \\
\text { benachteiligten und unterrepräsentierten Menschen aufzunehmen. }\end{array}$ \\
\hline Status & 2009 gestartet; laufend. \\
\hline \multirow[t]{2}{*}{ Kurzprofil des Programms } & $\begin{array}{l}\text { Der Förderungsfonds für das Unternehmertum wurde durch das Ministerium für Soziale Sicherheit und Arbeit, das } \\
\text { Finanzministerium und INVEGA (der für die Entwicklung von KMU verantwortlichen Agentur) eingerichtet. Das Programm } \\
\text { bietet Mikrokredite in Höhe von maximal } 25000 \text { EUR für Existenzgründer und selbstständig Erwerbstätige, die seit weniger } \\
\text { als einem Jahr ein Unternehmen führen. Die Mikrokredite werden als Teil eines Pakets gewährt, das aus ergänzenden } \\
\text { Unterstützungsleistungen besteht, darunter kostenfreie Schulungen, Unternehmensberatung und zusätzliche finanzielle } \\
\text { Förderung durch Zinszuschüsse und Teilzuschüsse für Mitarbeiter (für die Start-ups, die Arbeitsplätze schaffen). Das Programm } \\
\text { gewährt außerdem Garantien von bis zu } 80 \% \text { des Darlehenswertes. }\end{array}$ \\
\hline & $\begin{array}{l}\text { Verwaltet wird das EPF-Programm von INVEGA in Zusammenarbeit mit der Litauischen Zentralen Kreditgenossenschaft (LZK), } \\
\text { die die Funktion eines Finanzintermediärs übernimmt. Die Darlehen werden durch Kreditgenossenschaften gewährt, und die LZK } \\
\text { unterhält Partnerschaften mit } 15 \text { Schulungsanbietern, die kostenfreie Schulungen und Unternehmensberatungsdienste für die } \\
\text { Darlehensempfänger durchführen. }\end{array}$ \\
\hline Hintergrund & $\begin{array}{l}\text { Der Zugang zu Finanzierung stellt für Existenzgründer in Litauen seit geraumer Zeit ein Problem dar, das sich durch die } \\
\text { schwierigen wirtschaftlichen Umstände nach der Wirtschaftskrise weiter zuspitzte. Existenzgründer aus den prioritären Gruppen, } \\
\text { die von dem Programm bevorzugt werden, sind beim Zugang zu Finanzierung mit größeren Herausforderungen konfrontiert als } \\
\text { Unternehmer aus den allgemeinen Gruppen, die eine Unternehmensgründung anstreben. Dies begründet den prioritären Status } \\
\text { dieser Zielgruppen im EPF-Programm. }\end{array}$ \\
\hline Dauer der Unterstützung & $\begin{array}{l}\text { Darlehen werden innerhalb von vier bis sechs Wochen gewährt, Garantien innerhalb von acht Wochen. Für die gewährten } \\
\text { Darlehen ist keine Höchstlaufzeit festgelegt. } 64 \text { Schulungsstunden werden angeboten. }\end{array}$ \\
\hline \multirow[t]{4}{*}{ Größte Herausforderungen } & $\begin{array}{l}\text { 1. Zum Zeitpunkt der Einrichtung des EPF im Jahr } 2009 \text { erwog man ursprünglich die Strategie, die Darlehen teilweise (zu } \\
30 \% \text { ) in eine Beihilfe umzuwandeln, um die Darlehenskosten der Teilnehmer zu reduzieren. Allerdings war es aufgrund von } \\
\text { Verordnungen der Europäischen Union nicht möglich, einen Teil eines Darlehens in eine Beihilfe umzuwandeln. Darum wurden } \\
\text { andere Möglichkeiten der finanziellen Unterstützung untersucht. Das Wirtschaftsministerium einigte sich mit dem Ministerium } \\
\text { für soziale Sicherheit und Arbeit auf die Einführung von Zinszuschüssen, die schließlich } 2012 \text { stattfand. }\end{array}$ \\
\hline & $\begin{array}{l}\text { 2. Die Einrichtung des EPF-Programms dauerte länger als erwartet, zum Teil aufgrund der Komplexität der Verordnungen der } \\
\text { Europäischen Union im Zusammenhang mit dem Europäischen Fonds für regionale Entwicklung. }\end{array}$ \\
\hline & $\begin{array}{l}\text { 3. Die Umstände nach der Wirtschaftskrise bedeuteten, dass die Bedingungen für Unternehmensgründungen schwieriger waren } \\
\text { als vorausgesehen und die anfängliche Nachfrage nach Darlehen nicht so hoch war wie erwartet. }\end{array}$ \\
\hline & 4. Die Finanzierung stellt eine Herausforderung dar, insbesondere die Sicherung von ausreichenden Mitteln aus dem Privatsektor. \\
\hline Auswirkungen & $\begin{array}{l}\text { Das Programm hat die meisten seiner Ziele für den Zeitraum 2010-2014 übertroffen, insbesondere hinsichtlich der } \\
\text { Unterstützung, die den prioritären Gruppen geleistet wurde. Insgesamt haben } 4205 \text { Menschen an Schulungen teilgenommen } \\
\text { und } 4117 \text { haben diese abgeschlossen. } 1017 \text { Darlehen wurden gewährt, davon } 479 \text { an prioritäre Gruppen ( } 47 \% \text { ). Die } \\
\text { Darlehensempfänger haben } 1758 \text { neue Arbeitsplätze geschaffen. }\end{array}$ \\
\hline Durchschnittliche jährliche Kosten & 3,62 Millionen EUR \\
\hline Kosten pro Teilnehmer (“) & 3500 (berechnet anhand der Anzahl der Menschen, die die Schulungen begonnen haben). \\
\hline Finanzierungsquellen & Europäischer Sozialfonds, Europäischer Fonds für regionale Entwicklung, nationale Regierung, Mittel aus dem Privatsektor \\
\hline
\end{tabular}

$\left(^{*}\right)$ Beim Vergleich der Kosten pro Teilnehmer der verschiedenen Programme sollten die Dauer der Programme und die Unterschiede in den angebotenen Dienstleistungen beachtet werden. 


\section{Niederlande}

\begin{tabular}{|c|c|}
\hline \multicolumn{2}{|r|}{ Sozialhilfe für selbstständig Erwerbstätige (BBZ) } \\
\hline Zielgruppe & Arbeitslose und Kleinunternehmer, die vor dem Konkurs stehen \\
\hline Geleistete Unterstützung & Finanzierung (Beihilfen, Darlehen, Zuwendungen), Coaching und Mentoring, Schulungen, Unternehmensberatung \\
\hline Ziel & $\begin{array}{l}\text { BBZ zielt darauf ab, Sozialhilfeempfänger bei der Gründung eines kleinen Unternehmens und der Aufnahme einer selbstständigen } \\
\text { Erwerbstätigkeit zu unterstützen. }\end{array}$ \\
\hline Status & 1996 gestartet; laufend. \\
\hline \multirow[t]{2}{*}{ Kurzprofil des Programms } & $\begin{array}{l}\text { BBZ bietet ein integriertes Paket an Unterstützungsleistungen für Unternehmer vor und nach der Gründung. Das BBZ-Programm } \\
\text { besteht aus acht Stufen, darunter Aufnahme, Schulung, Entwicklung eines Geschäftsplans und Finanzierung. Die Teilnehmer } \\
\text { müssen nicht alle Stufen durchlaufen, doch die meisten entscheiden sich dafür. In der Regel dauern die der Gründung } \\
\text { vorangehenden Stufen neun Monate. Nach der Unternehmensgründung können die Teilnehmer zwölf Monate lang ein Mentoring } \\
\text { in Anspruch nehmen. }\end{array}$ \\
\hline & $\begin{array}{l}\text { Das Ministerium für Soziales und Beschäftigung überwacht das Programm, das in erster Linie durch die } \\
\text { kommunalen Abteilungen für Soziales und Beschäftigung umgesetzt wird. Privatwirtschaftliche und halbstaatliche } \\
\text { Wirtschaftsförderungsdienstleister sowie mit öffentlicher Beteiligung arbeitende Einrichtungen werden durch die } \\
\text { Stadtverwaltungen mit der Bereitstellung der Dienstleistungen beauftragt. Die Stadtverwaltungen entscheiden nach eigenem } \\
\text { Ermessen, wie sie die Dienstleister für Schulungen und Unternehmensberatung beauftragen. Viele Stadtverwaltungen nutzen } \\
\text { Festaufträge (durch beschränkte und auch manchmal offene Ausschreibungsverfahren) mit einem oder zwei Dienstleistern für } \\
\text { einen festgelegten Zeitraum. }\end{array}$ \\
\hline Hintergrund & $\begin{array}{l}\text { Das Programm startete 1996, als die Arbeitslosigkeit hoch war und weiter stieg. Das Unternehmertum wurde als produktive } \\
\text { Methode betrachtet, Arbeitslose wieder in die erwerbstätige Bevölkerung zu integrieren, insbesondere diejenigen, die } \\
\text { Sozialhilfeleistungen bezogen. }\end{array}$ \\
\hline Dauer der Unterstützung & $\begin{array}{l}\text { Die Unterstützung vor der Unternehmensgründung dauert in der Regel neun Monate an; Darlehen haben Laufzeiten von } \\
\text { bis zu fünf Jahren; Zuwendungen können bis zu drei Jahre lang geleistet werden; Unterstützung in Form von Coaching und } \\
\text { Unternehmensberatung nach der Gründung wird ein Jahr lang angeboten. }\end{array}$ \\
\hline \multirow[t]{4}{*}{ Größte Herausforderungen } & $\begin{array}{l}\text { 1. Die Preisgestaltung für die Wirtschaftsförderungsdienste, die den Klienten angeboten werden, ist schwierig, sodass die } \\
\text { Programmleiter Mühe hatten, Partner zu gewinnen und die Bereitstellung dieser Unterstützung zu organisieren. }\end{array}$ \\
\hline & 2. Der Aufbau und die Aufrechterhaltung von Beziehungen mit einer abnehmenden Anzahl lokaler Dienstleister. \\
\hline & $\begin{array}{l}\text { 3. Hauptsächlich für die Umsetzung zuständig sind die Stadtverwaltungen, die wenig Erfahrung mit der Koordinierung derartiger } \\
\text { Programme haben. Die Mitarbeiter haben Schwierigkeiten, sich an einen wechselnden Kundenstamm und ein sich entwickelndes } \\
\text { Wirtschaftsumfeld anzupassen. }\end{array}$ \\
\hline & $\begin{array}{l}\text { 4. Die Programmteilnehmer sehen sich einem möglichen Verlust ihrer Sozialleistungen gegenüber, was von der Teilnahme } \\
\text { abschreckt. }\end{array}$ \\
\hline \multirow[t]{2}{*}{ Auswirkungen } & $\begin{array}{l}\text { Im Durchschnitt steigen jedes Jahr zwischen } 1800 \text { und } 2400 \text { Menschen in das Projekt ein. Davon schließen } 900 \text { bis } 1200 \text { das } \\
\text { Unterstützungsprogramm ab und gründen ein Unternehmen. Seit 2009/2010 blieben } 72 \% \text { der Teilnehmer drei Jahre nach } \\
\text { ihrem Einstieg in das Programm erwerbstätig. Davon fanden } 20 \% \text { eine abhängige Erwerbstätigkeit und } 80 \% \text { setzten ihre } \\
\text { unternehmerischen Bemühungen fort. }\end{array}$ \\
\hline & $\begin{array}{l}\text { Laut Schätzungen durch Evaluierungen beläuft sich der gesellschaftliche Nettobeitrag jedes durch das Programm unterstützten } \\
\text { Start-ups auf fast } 20000 \text { EUR. }\end{array}$ \\
\hline Durchschnittliche jährliche Kosten & 40,4 Millionen EUR \\
\hline Kosten pro Teilnehmer (*) & 33700 EUR \\
\hline Finanzierungsquellen & $\begin{array}{l}\text { Nationale Regierung (Ministerium für Soziales und Beschäftigung); Europäischer Sozialfonds; sonstige EU-Mittel (ausschließlich } \\
\text { Sonderprojekte) }\end{array}$ \\
\hline
\end{tabular}

(*) Beim Vergleich der Kosten pro Teilnehmer der verschiedenen Programme sollten die Dauer der Programme und die Unterschiede in den angebotenen Dienstleistungen beachtet werden. 


\section{Polen}

\begin{tabular}{|c|c|}
\hline \multicolumn{2}{|r|}{ Reifer Unternehmer } \\
\hline Zielgruppe & Arbeitslose über 50 \\
\hline Geleistete Unterstützung & Finanzierung (Beihilfen und Überbrückungshilfe), Schulungen, Coaching und Mentoring, Unternehmensberatung, Netzwerkarbeit. \\
\hline Ziel & $\begin{array}{l}\text { Die Unterstützung Älterer bei der Gründung eines Unternehmens, damit sie auf dem Arbeitsmarkt aktiv bleiben können. } \\
\text { Weiterhin zielt das Projekt darauf ab, eine positivere Einstellung gegenüber Älteren in der Gesellschaft zu schaffen und lokal für } \\
\text { mehr Unternehmertum zu sorgen. }\end{array}$ \\
\hline Status & 2009-2010; abgeschlossen. \\
\hline \multirow[t]{2}{*}{ Kurzprofil des Programms } & $\begin{array}{l}\text { Das polnische Projekt „Reifer Unternehmer“ wurde vom kommunalen Arbeitsamt in Gdansk entwickelt und möchte Älteren } \\
\text { zwischen } 50 \text { und } 64 \text { Jahren, die ein Unternehmen gründen möchten, Orientierungshilfen und finanzielle Unterstützung bieten. Als } \\
\text { Kandidaten infrage kommen Arbeitslose, Rentner oder Menschen, die aus der abhängigen in die selbstständige Erwerbstätigkeit } \\
\text { wechseln möchten. }\end{array}$ \\
\hline & $\begin{array}{l}\text { Das Projekt umfasst } 150 \text { Schulungsstunden in acht Modulen. Nach Abschluss der Schulungen kann man sich um eine } \\
\text { finanzielle Unterstützung bewerben. Diese besteht aus einer Beihilfe (bis zu } 40000 \text { PLN bzw. etwa } 9650 \text { EUR) und einer } \\
\text { monatlichen Überbrückungshilfe (1250 PLN bzw. etwa } 300 \text { EUR). Die Teilnehmer können bis zu vier Stunden individueller } \\
\text { Beratung rund um die Entwicklung von Geschäfts- und Finanzplänen sowie zwölf Stunden individueller Unterstützung nach der } \\
\text { Unternehmensgründung in Anspruch nehmen. Das Projekt fördert außerdem die Entwicklung von Netzwerken, indem Teilnehmer } \\
\text { in kleine Gruppen eingeteilt und Betreuer und Ausbilder ermutigt werden, ihre Netzwerke für die Teilnehmer zu öffnen. }\end{array}$ \\
\hline Hintergrund & $\begin{array}{l}\text { Ältere in Polen haben Schwierigkeiten auf dem Arbeitsmarkt, weil sie stärker als jüngere Arbeitskräfte gefährdet sind, wenn } \\
\text { Unternehmen Personal abbauen, und häufig zugunsten jüngerer Menschen übersehen werden, wenn sie eine Beschäftigung } \\
\text { suchen. Aber diese Altersgruppe hat noch viel zur Gesellschaft und zum Arbeitsmarkt beizutragen. Häufig müssen viele von } \\
\text { innen zudem weiter erwerbstätig bleiben, da sie nur über geringe Ersparnisse für den Ruhestand verfügen. }\end{array}$ \\
\hline Dauer der Unterstützung & Zwölfmonatige Unterstützung \\
\hline \multirow[t]{3}{*}{ Größte Herausforderungen } & $\begin{array}{l}\text { 1. Die Auswahl der Teilnehmer war schwierig, da viele eingereichte Geschäftsideen nicht realisierbar waren und wenig Aussicht } \\
\text { hatten, als Grundlage für ein Unternehmen zu dienen. }\end{array}$ \\
\hline & $\begin{array}{l}\text { 2. Die Unterstützung der Teilnehmer beim Erwerb der zur Umsetzung ihrer Idee notwendigen Fähigkeiten, da es häufig eine } \\
\text { Diskrepanz zwischen den Fähigkeiten und Qualifikationen und der Geschäftsidee gab. }\end{array}$ \\
\hline & 3. Die Sicherung einer Finanzierung für die Fortführung des Projekts. \\
\hline Auswirkungen & $\begin{array}{l}\text { "Reifer Unternehmer“ förderte die Gründung } 33 \text { neuer Unternehmen in der Stadt Gdansk und in Powiat Gda囚ski, was sich positiv } \\
\text { auf die lokale Wirtschaft ausgewirkt hat. Alle neu gegründeten Unternehmen sind immer noch aktiv, einige davon wachsen sogar } \\
\text { so stark, dass weitere Vollzeitstellen geschaffen wurden. }\end{array}$ \\
\hline Durchschnittliche jährliche Kosten & 240265 EUR \\
\hline Kosten pro Teilnehmer ( & 8009 EUR \\
\hline Finanzierungsquellen & Europäischer Sozialfonds (85\%); nationale Regierung (15\%) \\
\hline
\end{tabular}

(*) Beim Vergleich der Kosten pro Teilnehmer der verschiedenen Programme sollten die Dauer der Programme und die Unterschiede in den angebotenen Dienstleistungen beachtet werden. 


\section{Slowenien}

\begin{tabular}{|c|c|}
\hline \multicolumn{2}{|r|}{ Unternehmerisch in die Geschäftswelt } \\
\hline Zielgruppe & Arbeitslose unter 35 Jahre mit einem abgeschlossenen grundständigen oder postgradualen Studium. \\
\hline Geleistete Unterstützung & Finanzierung (Zuwendungen), Schulungen, Coaching, Unternehmensberatung \\
\hline Ziel & $\begin{array}{l}\text { Hilfe für hoch qualifizierte Arbeitslose unter } 35 \text { Jahren beim Einstieg in die selbstständige Erwerbstätigkeit oder bei der } \\
\text { Aufnahme einer abhängigen Erwerbstätigkeit. }\end{array}$ \\
\hline Status & Pilotprojekt (2009-2012); nationale Projekte (2013 bis heute) \\
\hline Kurzprofil des Programms & $\begin{array}{l}\text { Das Projekt soll unter 35-Jährigen mit einem hohen Bildungsstand bei der Unternehmensgründung oder bei der Aufnahme einer } \\
\text { abhängigen Erwerbstätigkeit unterstützen. Die Teilnehmer werden als öffentliche Bedienstete eingestellt und erhalten vier Monate } \\
\text { lang ein Monatsgehalt von } 798 \text { EUR. Dabei handelt es sich im Wesentlichen also um eine Zuwendung. Anstatt zu arbeiten, } \\
\text { erhalten die Teilnehmer unternehmerische Schulungen und individuelles Coaching, Mentoring sowie Unternehmensberatung } \\
\text { durch regionale Entwicklungsagenturen, die ihnen bei der Unternehmensgründung helfen. Die Schulungen und das Coaching } \\
\text { werden von Mitarbeitern dieser Entwicklungszentren und von Unternehmensberatern durchgeführt. Außerdem haben die } \\
\text { Teilnehmer für ein Jahr ab Unternehmensgründung Zugang zu Unternehmensberatung. Das Projekt wird in drei Phasen } \\
\text { umgesetzt: ein regionales Pilotprojekt und zwei nationale Projekte in allen Regionen. }\end{array}$ \\
\hline Hintergrund & $\begin{array}{l}\text { Mit dem Projekt soll die hohe Arbeitslosenquote unter den jungen Menschen angegangen werden, die vor einem mangelnden } \\
\text { Arbeitsplatzangebot stehen. Die Arbeitslosenquote der jungen Menschen in Slowenien stieg von 10\% im Jahr } 2009 \text { rapide auf } \\
25 \% \text { im Jahr } 2013 \text { an. Gleichzeitig vermittelte das Bildungssystem nicht die Fähigkeiten und Kenntnisse, die arbeitslose, aber } \\
\text { hochgebildete Menschen benötigten, um ihre Geschäftsideen zu verfolgen und sich selbstständig zu machen. }\end{array}$ \\
\hline Dauer der Unterstützung & $\begin{array}{l}\text { Schulungen und Zuwendungen für bis zu vier Monate; Unternehmensberatungsleistungen für ein Jahr nach der } \\
\text { Unternehmensgründung. }\end{array}$ \\
\hline \multirow[t]{2}{*}{ Größte Herausforderungen } & $\begin{array}{l}\text { 1. Es ist schwierig, eine angemessene Anzahl hoch qualifizierter Bewerber zu finden. Während des Auswahlverfahrens werden } \\
\text { abgelehnte Bewerber aus den Vorjahren wieder eingeladen, wenn es an qualifizierten Bewerbern mangelt. }\end{array}$ \\
\hline & 2. Die Entwicklung einer Schulungsmethode ist schwierig. Die aktuelle Methode wurde anhand von Experimenten entworfen. \\
\hline Auswirkungen & $\begin{array}{l}\text { Überwachungsdaten deuten darauf hin, dass } 134 \text { (56\%) der Teilnehmer am ersten nationalen Projekt (d. h. im Jahr 2013) } \\
\text { erfolgreich ein Unternehmen gegründet oder eine abhängige Erwerbstätigkeit gefunden haben. }\end{array}$ \\
\hline Durchschnittliche jährliche Kosten & Regional: 151225 EUR; national (2013): 3816534 EUR; national (2014 bis heute): 4702038 EUR \\
\hline Kosten pro Teilnehmer ( $\left.{ }^{*}\right)$ & Pilotprojekt: 15122 EUR; nationale Phase 1: 15266 EUR; nationale Phase 2: 13061 EUR \\
\hline \multirow[t]{3}{*}{ Finanzierungsquellen } & $\begin{array}{l}\text { Regional: Europäischer Fonds für regionale Entwicklung (28\%); Stadtverwaltung von Zagorje ob Savi (15\%); lokale } \\
\text { Gebietskörperschaften }(57 \%)\end{array}$ \\
\hline & $\begin{array}{l}\text { Nationale Phase } 1(2013) \text { : Europäischer Sozialfonds (85\%); slowenisches Ministerium für Arbeit, Familie, Soziales und } \\
\text { Gleichstellung }(15 \%)\end{array}$ \\
\hline & $\begin{array}{l}\text { Nationale Phase } 2 \text { (2014 bis heute): Europäischer Sozialfonds ( } 85 \%) \text {; slowenisches Ministerium für Arbeit, Familie, Soziales und } \\
\text { Gleichstellung }(15 \%)\end{array}$ \\
\hline
\end{tabular}

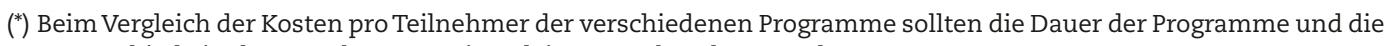
Unterschiede in den angebotenen Dienstleistungen beachtet werden. 


\section{Spanien}

\begin{tabular}{|c|c|}
\hline \multicolumn{2}{|r|}{ Startrampen für Beschäftigung und Unternehmertum } \\
\hline Zielgruppe & Langzeitarbeitslose (d. h. seit mehr als zwölf Monaten). \\
\hline Geleistete Unterstützung & Coaching und Mentoring, Unternehmensberatung, Schulungen, Räumlichkeiten. \\
\hline Ziel & $\begin{array}{l}\text { Dieses Programm zielt darauf ab, die Beschäftigungsfähigkeit seiner Teilnehmer durch Schulungen, die Stärkung des } \\
\text { Selbstwertgefühls, den Aufbau von Netzwerken und die Entwicklung unternehmerischer Fähigkeiten zu verbessern. Ein weiteres } \\
\text { Ziel ist die Intensivierung der Verbindungen zwischen Wirtschaftsförderungsdienstleistern. }\end{array}$ \\
\hline Status & $\begin{array}{l}\text { Pilotprojekt (April 2013-Dezember 2013). Das Pilotprojekt hat sich inzwischen zu einem Netzwerk von „Startrampen“ entwickelt } \\
\text { und läuft nach wie vor. }\end{array}$ \\
\hline \multirow[t]{3}{*}{ Kurzprofil des Programms } & $\begin{array}{l}\text { Das Programm besteht aus einem Netzwerk von „Startrampen“, die Langzeitarbeitslosen dabei helfen sollen, durch die Gründung } \\
\text { eines Unternehmens oder die Aufnahme einer abhängigen Erwerbstätigkeit wieder auf den Arbeitsmarkt zurückzukehren. } \\
\text { Jede der „Startrampen“ ist ein strukturiertes Projekt, das an drei Tagen in der Woche Gruppen-Coachings und Schulungen } \\
\text { und während der beiden übrigen Tage Unternehmensberatung anbietet. Ergänzt wird dies durch Schulungen, Workshops, } \\
\text { Geschäftsbesuche und Netzwerkveranstaltungen. }\end{array}$ \\
\hline & $\begin{array}{l}\text { Die „Startrampen“ sind teilautonom und werden jeweils von einem Betreuer geleitet. Jede von ihnen hat die Möglichkeit, die } \\
\text { geleistete Unterstützung auf die Bedürfnisse der Teilnehmer in ihrer Region zuzuschneiden, und wird ihrerseits von der Zentrale } \\
\text { unterstützt. Die einzigen bezahlten Mitarbeiter sind die Betreuer - die anderen Unterstützungsgeber sind Freiwillige. Die Betreuer } \\
\text { sind für die Weiterentwicklung und Umsetzung des Programms und für die Entwicklung und Verwaltung von Beziehungen mit } \\
\text { anderen Unterstützungsgebern verantwortlich. Sie werden vom LEES geschult. }\end{array}$ \\
\hline & LEES richtet außerdem Veranstaltungen für alle „Startrampen“ aus, unter anderem Netzwerkveranstaltungen und Konferenzen. \\
\hline Hintergrund & $\begin{array}{l}\text { Das Programm wurde ins Leben gerufen, um zur Unterstützung der Rückkehr von Arbeitslosen auf den Arbeitsmarkt beizutragen } \\
\text { und das soziale Stigma der Arbeitslosigkeit zu bekämpfen, indem Langzeitarbeitslosen geholfen wird, aktiv zu bleiben. Es wurde } \\
\text { in Anlehnung an einen Ansatz entworfen, der jungen Menschen Mitte bis Ende der 1980er beim Einstieg in den Arbeitsmarkt } \\
\text { helfen sollte. }\end{array}$ \\
\hline Dauer der Unterstützung & Sechs oder neun Monate, abhängig von der jeweiligen „Startrampe“. \\
\hline \multirow[t]{2}{*}{ Größte Herausforderungen } & $\begin{array}{l}\text { 1. Aufgrund des kleinen Haushalts kann nur ein Mitarbeiter pro „Startrampe“ eingestellt werden (der Betreuer), der für die } \\
\text { Entwicklung und Umsetzung des Programms sowie die Bereitstellung einer individuellen Unterstützung die volle Verantwortung } \\
\text { trägt. Die Arbeitsbelastung ist also immens, und es besteht die Gefahr, dass diese Aufgabe zu umfangreich für eine einzige } \\
\text { Person ist. }\end{array}$ \\
\hline & $\begin{array}{l}\text { 2. Die Messung der Auswirkungen gestaltet sich schwierig angesichts dessen, dass das Ziel des Programms darin besteht, einer } \\
\text { sehr benachteiligten Gruppe zu einer Beschäftigung zu verhelfen. Verschiedene Ergebnisse können als positiv gewertet werden, } \\
\text { aber es ist schwierig, das Programm direkt mit den Ergebnissen in Verbindung zu bringen. }\end{array}$ \\
\hline \multirow[t]{2}{*}{ Auswirkungen } & $\begin{array}{l}\text { Während der Pilotphase (April 2013-Dezember 2013) nahmen } 114 \text { Menschen an } 5 \text { „Startrampen“ teil: } 25 \% \text { von ihnen gründeten } \\
\text { ein Unternehmen, und } 63 \% \text { fanden eine abhängige Erwerbstätigkeit. Die übrigen } 22 \% \text { suchten entweder immer noch nach einer } \\
\text { Stelle oder meldeten sich zu einer Weiterbildung an, um ihre Aussichten bei der Arbeitssuche zu verbessern. }\end{array}$ \\
\hline & 2014 gab es 40 „Startrampen“. Man rechnete bis Ende 2015 mit der Verdoppelung dieser Anzahl. \\
\hline Durchschnittliche jährliche Kosten & 40000 EUR pro „Startrampe“, jedoch gibt es in Abhängigkeit der Dauer der geleisteten Unterstützung einige Abweichungen. \\
\hline Kosten pro Teilnehmer (") & 2000 EUR \\
\hline Finanzierungsquellen & Europäischer Sozialfonds (80\%); nationale Regierung (20\%) \\
\hline
\end{tabular}

$\left(^{*}\right)$ Beim Vergleich der Kosten pro Teilnehmer der verschiedenen Programme sollten die Dauer der Programme und die Unterschiede in den angebotenen Dienstleistungen beachtet werden. 


\section{Schweden}

\begin{tabular}{|c|c|}
\hline \multicolumn{2}{|r|}{ Botschafterinnen für Frauen } \\
\hline Zielgruppe & Frauen, Studentinnen \\
\hline Geleistete Unterstützung & Rollenvorbilder, Coaching \\
\hline Ziel & $\begin{array}{l}\text { Das Botschafterinnen-Projekt bot eine Struktur für Unternehmerinnen, um ihre unternehmerischen Erfahrungen mit anderen zu } \\
\text { teilen und dadurch die Sichtbarkeit von Unternehmerinnen zu erhöhen, durch persönliche Geschichten und Rollenvorbilder zum } \\
\text { Unternehmertum zu inspirieren, Frauen die Identifikation mit Unternehmerinnen als Rollenvorbilder zu erleichtern, mehr Frauen } \\
\text { dazu zu ermutigen, das Unternehmertum als potenzielle berufliche Laufbahn zu betrachten und den Erfahrungsaustausch zu } \\
\text { fördern. }\end{array}$ \\
\hline Status & 2008-2014; abgeschlossen. \\
\hline \multirow[t]{2}{*}{ Kurzprofil des Programms } & $\begin{array}{l}\text { Das Botschafterinnen-Projekt warb bei Frauen für das Unternehmertum, indem es Unternehmerinnen als Rollenvorbilder } \\
\text { einsetzte. Diese Botschafterinnen nahmen an Veranstaltungen teil und hielten Präsentationen über ihre Geschichte und } \\
\text { das Unternehmertum im Allgemeinen. Regionale Koordinatoren übernahmen die Verwaltung für die Botschafterinnen und } \\
\text { organisierten den Zeitplan für jede von innen. Ein besonderer Schwerpunkt des Programms war die Kontaktaufnahme zu Schulen } \\
\text { und Universitäten, um das Unternehmertum bei Studentinnen zu fördern. }\end{array}$ \\
\hline & $\begin{array}{l}\text { Zusätzlich zu diesen Werbeaktivitäten waren viele der Botschafterinnen als Betreuer und Mentoren für Unternehmerinnen } \\
\text { tätig. Dies wurde durch das Programm gefördert und wurde } 2010 \text { Teil des offiziellen Angebots. Die Termine wurden individuell } \\
\text { vereinbart oder unter www.ambassadorer.se gebucht. }\end{array}$ \\
\hline Hintergrund & $\begin{array}{l}\text { Dieses Projekt war Teil eines politischen Maßnahmenpakets, um für das weibliche Unternehmertum zu werben. Umfragen } \\
\text { deuteten darauf hin, dass kaum ein Bewusstsein für den Beitrag von Unternehmerinnen zur Gesellschaft vorhanden ist und dass } \\
\text { wenige Studenten eine berufliche Laufbahn als Selbstständige in Betracht zogen. }\end{array}$ \\
\hline Dauer der Unterstützung & $\begin{array}{l}\text { Die Botschaften warben bei Veranstaltungen und bei Studentinnen für das weibliche Unternehmertum. Einige Frauen } \\
\text { entwickelten Coaching- und Mentoring-Beziehungen mit inrem Mentor. Die Bedingungen und die Dauer dieser Beziehungen } \\
\text { waren Vereinbarungssache beider Parteien. }\end{array}$ \\
\hline \multirow[t]{3}{*}{ Größte Herausforderungen } & $\begin{array}{l}\text { 1. Die freiwilligen Botschafterinnen wurden zuweilen durch die hohen Opportunitätskosten abgeschreckt, die eine Tätigkeit als } \\
\text { Rollenvorbild verursachte, insbesondere in ländlichen Regionen, wo sie viel reisen mussten. }\end{array}$ \\
\hline & $\begin{array}{l}\text { 2. Die regionalen Koordinatoren verfügten nicht über einen Mechanismus, um freiwillige Botschafterinnen, die ungeeignet waren, } \\
\text { abzulehnen. }\end{array}$ \\
\hline & $\begin{array}{l}\text { 3. Das Programm musste sich vielen Fragen darüber stellen, ob ein derartiges „weiches“ politisches Instrument Auswirkungen } \\
\text { erzielen kann. }\end{array}$ \\
\hline Auswirkungen & $\begin{array}{l}\text { Vom Start (Mai 2008) bis zum Abschluss (Dezember 2014) erreichten die Botschafterinnen des Programms mehr als } \\
170000 \text { Menschen mit etwa } 11000 \text { Aktivitäten. Laut Befragungen der Teilnehmer interessierten sich } 50 \% \text { von ihnen dank der } \\
\text { Botschafterinnen stärker für das Unternehmertum. }\end{array}$ \\
\hline Durchschnittliche jährliche Kosten & 1,72 Millionen EUR (für die letzte Phase von 2011 bis 2014) \\
\hline Kosten pro Teilnehmer ( & 217 EUR (pro erreichten Teilnehmer) \\
\hline Finanzierungsquellen & Nationale Regierung \\
\hline
\end{tabular}

(*) Beim Vergleich der Kosten pro Teilnehmer der verschiedenen Programme sollten die Dauer der Programme und die Unterschiede in den angebotenen Dienstleistungen beachtet werden. 


\section{Vereinigtes Königreich}

\begin{tabular}{|c|c|}
\hline \multicolumn{2}{|r|}{ Access to Work } \\
\hline Zielgruppe & Menschen mit gesundheitlichen Problemen/Behinderungen, die ihre Erwerbsfähigkeit beeinträchtigen. \\
\hline Geleistete Unterstützung & Finanzierung (Beihilfen) \\
\hline Ziel & $\begin{array}{l}\text { Verschiedene Unterstützungsleistungen zur Arbeitsplatzanpassung, um Menschen mit Behinderungen die Gründung eines } \\
\text { Unternehmens oder die Tätigkeit als Angestellter zu erleichtern. }\end{array}$ \\
\hline Status & 1984 gestartet; laufend. \\
\hline Kurzprofil des Programms & $\begin{array}{l}\text { Das Programm bietet Beratung und praktische Unterstützung für Menschen mit Behinderungen und ihre Arbeitgeber, damit diese } \\
\text { Arbeitshindernisse bewältigen können. Access to Work zahlt über Jobcentre Plus Beihilfen aus, um die Kosten für angemessene } \\
\text { Arbeitsplatzanpassungen für Menschen mit Behinderungen (sowohl für Angestellte als auch für selbstständig Erwerbstätige) } \\
\text { zu decken. Zudem können andere, individuell angepasste Unterstützungsleistungen erbracht werden, zum Beispiel Beihilfen } \\
\text { für spezielle Hilfsmittel und Ausrüstung, Anpassungen von Räumlichkeiten und Vorkehrungen für den Arbeitsweg. Access to } \\
\text { Work erstattet Arbeitgebern und selbstständig Erwerbstätigen die Kosten für Maßnahmen, die sie ergreifen, um Menschen mit } \\
\text { Behinderungen am Arbeitsplatz zu helfen. Die Höhe der Erstattungen hängt von den jeweiligen Umständen ab (z. B. der Art } \\
\text { der ergriffenen Maßnahmen, dem Grad der Behinderung der Person). Menschen, die sich für eine Unternehmensgründung } \\
\text { interessieren, werden außerdem durch den Zugang zur New Enterprise Allowance (Zuwendung für neue Unternehmen) } \\
\text { unterstützt. Die Mittel, die von Access to Work gezahlt werden, haben keinen Einfluss auf andere bezogene Leistungen. }\end{array}$ \\
\hline Hintergrund & $\begin{array}{l}\text { Das Programm fördert die Umsetzung des Disability Discrimination Act (Gesetz zur Diskriminierung aufgrund einer } \\
\text { Behinderung). Sein Zweck besteht darin, für angemessene Anpassungen des Arbeitsplatzes von Menschen mit Behinderungen } \\
\text { zu sorgen. Das Programm wird als wirksame Möglichkeit betrachtet, Menschen mit Behinderungen zur Aufnahme einer } \\
\text { Erwerbstätigkeit zu ermutigen und sie dabei zu unterstützen sowie die Beteiligungsquoten zu erhöhen. }\end{array}$ \\
\hline Dauer der Unterstützung & Die Dauer der Unterstützung ist von Fall zu Fall unterschiedlich. \\
\hline \multirow[t]{3}{*}{ Größte Herausforderungen } & 1. Geringe und lückenhafte Bekanntheit aufgrund fehlender dedizierter Aufklärungsprogramme. \\
\hline & $\begin{array}{l}\text { 2. Die Teilnehmer gaben häufig an, dass sie Schwierigkeiten hatten, ihre besonderen Bedürfnisse und die Maßnahmen, die ihnen } \\
\text { helfen würden, zu erklären. }\end{array}$ \\
\hline & $\begin{array}{l}\text { 3. Die Unterstützung von selbstständig Erwerbstätigen ist schwierig, da es sich bei Access to Work um ein Erstattungsprogramm } \\
\text { handelt, bei dem in den meisten Fällen anfängliche Investitionen geleistet werden müssen. }\end{array}$ \\
\hline \multirow[t]{3}{*}{ Auswirkungen } & $\begin{array}{l}\text { Im Jahr } 2012 \text { half Access to Work mehr als } 30000 \text { Menschen, einen Arbeitsplatz zu finden oder zu behalten. Davon waren etwa } \\
4500 \text { in Kleinunternehmen tätig. } 45 \% \text { der Empfänger wären arbeitslos, hätte man nicht die durch das Programm finanzierten } \\
\text { Anpassungen vorgenommen. }\end{array}$ \\
\hline & Zusammen mit der New Enterprises Allowance trug Access to Work im Jahr 2012 zu rund 8000 Unternehmensgründungen bei. \\
\hline & $\begin{array}{l}\text { Das Programm erzielte einen klaren finanziellen Ertrag für die Investition des Finanzministeriums. Mit dem Betrag, der an } \\
\text { Arbeitslosenunterstützung gespart wurde, erzielte man einen Ertrag von 1,48 EUR für jeden durch das Finanzministerium } \\
\text { investierten Euro. }\end{array}$ \\
\hline Durchschnittliche jährliche Kosten & 138 Millionen EUR \\
\hline Kosten pro Teilnehmer (") & 2875 EUR \\
\hline Finanzierungsquellen & Nationale Regierung (Ministerium für Arbeit und Renten) \\
\hline
\end{tabular}

(*) Beim Vergleich der Kosten pro Teilnehmer der verschiedenen Programme sollten die Dauer der Programme und die Unterschiede in den angebotenen Dienstleistungen beachtet werden. 


\section{Vereinigtes Königreich}

\begin{tabular}{|c|c|}
\hline \multicolumn{2}{|r|}{ Prowess } \\
\hline Zielgruppe & Frauen \\
\hline Geleistete Unterstützung & Qualitätszertifizierungen für Wirtschaftsförderungsdienste für Frauen \\
\hline Ziel & $\begin{array}{l}\text { Prowess wurde } 2002 \text { damit beauftragt, die erste nationale Strategie des Vereinigten Königreichs für das weibliche } \\
\text { Unternehmertum zu erarbeiten und Qualitätsstandards für die Unterstützung von Unternehmen von Frauen zu entwickeln. }\end{array}$ \\
\hline Status & Prowess nahm seine Tätigkeit im Jahr 2002 auf, erfand sich aber 2012 als Sozialunternehmen neu; laufend. \\
\hline \multirow[t]{2}{*}{ Kurzprofil des Programms } & $\begin{array}{l}\text { Bei Prowess handelt es sich um ein Mitgliedschaftsnetzwerk aus Organisationen, die eine frauenfreundliche Unterstützung für } \\
\text { die Unternehmensgründung im gesamten Vereinigten Königreich leisten. Das Programm wurde } 2002 \text { als National Association } \\
\text { for the Promotion of Women's Enterprise (Nationaler Verband zur Förderung des weiblichen Unternehmertums) mit der } \\
\text { Unterstützung der nationalen Regierung im Vereinigten Königreich eingerichtet. In seiner Hochphase hatte Prowess mehr als } \\
350 \text { Mitgliedsorganisationen, die insgesamt über } 100000 \text { Unternehmensinhaberinnen unterstützten. Nachdem die öffentlichen } \\
\text { Mittel } 2012 \text { ausliefen, erwarb der ehemalige Geschäftsführer die Vermögenswerte der Organisation, die anschließend als } \\
\text { Sozialunternehmen neu aufgelegt wurde. }\end{array}$ \\
\hline & $\begin{array}{l}\text { Eine der Schlüsselinitiativen von Prowess war der Prowess Flagship Award. Mit dieser Auszeichnung sollten frauenfreundliche } \\
\text { Dienstleistungen zur Unternehmensförderung anerkannt werden. Der Flagship Award ist eine Auszeichnung auf der Grundlage } \\
\text { von zwölf Kriterien, anhand derer allgemeine und spezialisierte Anbieter einer Unternehmensförderung für Frauen bewertet } \\
\text { werden können. Prowess führte Bewertungen aller seiner Mitgliedsorganisationen durch. Zu jeder Bewertung gehörte ein } \\
\text { Standortbesuch, bei dem ein kleines Team von Prowess sich mit der Organisation und einigen ihrer Klienten traf, um die } \\
\text { Aktivitäten der Organisation anhand der zwölf Kriterien zu bewerten. }\end{array}$ \\
\hline Hintergrund & $\begin{array}{l}\text { Anfang der 2000er-Jahre erkannte die staatliche Einrichtung Small Business Service (Dienst für Kleinunternehmen), dass } \\
\text { Dienstleistungen zur Unternehmensförderung nicht genügend Frauen und andere soziale Zielgruppen (z. B. junge Menschen, } \\
\text { Zuwanderer, Menschen mit Behinderungen) erreichten. Prowess wurde } 2002 \text { eingerichtet, um die Herausforderungen im } \\
\text { Zusammenhang mit der Unterstützung des weiblichen Unternehmertums anzugehen. Das Programm wurde während eines } \\
\text { Zeitraums ins Leben gerufen, als die Regierung eine Politik verfolgte, gemäß derer spezialisierte Agenturen zur Unterstützung } \\
\text { bestimmter Zielgruppen eingerichtet werden sollten. }\end{array}$ \\
\hline Dauer der Unterstützung & k. A. \\
\hline \multirow[t]{2}{*}{ Größte Herausforderungen } & $\begin{array}{l}\text { 1. Sich ändernde Prioritäten der Regierung, unter anderem die Schließung der regionalen Entwicklungsagenturen, die bis zu } \\
\text { diesem Zeitpunkt aktiv an der Initiative mitgewirkt hatten. }\end{array}$ \\
\hline & $\begin{array}{l}\text { 2. Die Sicherung einer nachhaltigen Finanzierungsquelle. Der Verlust der Mittel aus dem Europäischen Sozialfonds und } \\
\text { die Streichung von privatwirtschaftlichen Mitteln aufgrund der Finanzkrise erforderten eine Änderung der Rechtsform der } \\
\text { Organisation. }\end{array}$ \\
\hline Auswirkungen & $\begin{array}{l}\text { Von den } 68 \text { Organisationen, die in der ersten Runde bewertet wurden, wurde sechs der Status „Flagship-Mitglied“ verliehen. } \\
\text { Ferner handelte es sich bei neun Mitgliedsorganisationen um Netzwerke, die anhand von leicht unterschiedlichen Kriterien } \\
\text { bewertet wurden. Zwei Mitgliedern wurde der Status „Flagship-Netzwerkmitglied“ verliehen. Aktuell gibt es } 24 \text { Organisationen, } \\
\text { die einen der Flagship Awards erhalten haben. }\end{array}$ \\
\hline Durchschnittliche jährliche Kosten & 700000 EUR \\
\hline Kosten pro Teilnehmer (“) & k. A. \\
\hline Finanzierungsquellen & $\begin{array}{l}\text { Von } 2002 \text { bis } 2009 \text { erhielt Prowess Mittel von der Europäischen Union über EQUAL und von der Phoenix-Stiftung. Seit } 2012 \text { ist } \\
\text { Prowess als Sozialunternehmen tätig und stützt sich auf Einnahmen aus Mitgliedsgebühren, Sonderveranstaltungen und } \\
\text { Beratung. }\end{array}$ \\
\hline
\end{tabular}

(*) Beim Vergleich der Kosten pro Teilnehmer der verschiedenen Programme sollten die Dauer der Programme und die Unterschiede in den angebotenen Dienstleistungen beachtet werden. 

TEIL II.

\section{Beispiele für bewährte Verfahren anhand von Programmen für ein integrationsförderndes Unternehmertum}





\title{
Unternehmensgründungsprogramm, Österreich
}

\begin{abstract}
Diese Fallstudie bietet ein Beispiel für ein integriertes Programm, das Arbeitslose bei der Unternehmensgründung unterstützt. Zu der Unterstützung gehören Schulungen, Beratung und finanzielle Unterstützung. Diese Fallstudie beschreibt die Ziele und den Hintergrund des Programms und gibt einen Überblick über seine zentralen Aktivitäten. Außerdem werden die Auswirkungen und die Herausforderungen präsentiert und die Voraussetzungen für die Übertragung dieses Verfahrens auf einen anderen Kontext erörtert.
\end{abstract}

Ziele

Das Ziel des Unternehmensgründungsprogramms (UGP) besteht in der Unterstützung von Arbeitslosen bei der Gründung eines eigenen Unternehmens. Das Programm wird vom Arbeitsmarktservice Österreich (AMS) durchgeführt und hilft Arbeitslosen dabei, durch die Gründung nachhaltiger neuer Unternehmen Arbeitsplätze für sich zu schaffen. Die Teilnehmer müssen beim AMS arbeitslos oder arbeitsuchend gemeldet sein und sich zudem für die Gründung eines Unternehmens interessieren, eine bestimmte Geschäftsidee haben und über einschlägige berufliche Erfahrungen und Fähigkeiten verfügen.

\section{Hintergrund}

Das Unternehmensgründungsprogramm wurde als Antwort auf die Herausforderungen entwickelt, mit denen der AMS bei der Unterstützungvon Arbeitslosen, die eine selbstständige Erwerbstätigkeit aufzunehmen beabsichtigten, konfrontiert war. Um eine Arbeitslosenunterstützung beziehen zu dürfen, müssen die Betroffenen erklären, dass sie für eine Beschäftigung zur Verfügung stehen. Allerdings liefen Arbeitslose, die sich für eine selbstständige Erwerbstätigkeit interessierten, Gefahr, ihre Arbeitslosenunterstützung zu verlieren, während sie an ihren Start-ups (d. h. vor der Unternehmensgründung) arbeiten, denn dies bedeutete, dass sie mit einiger Wahrscheinlichkeit nicht für eine Beschäftigung zu Verfügung standen. Somit schreckte das System der Arbeitslosenunterstützung Arbeitslose davon ab, eine selbstständige Erwerbstätigkeit als Möglichkeit der Rückkehr auf den Arbeitsmarkt in Betracht zu ziehen.

Zu Bewältigung dieses Problems setzte der AMS auf internationale Erfahrungen, um eine Lösung zu finden, die die Vorbereitung der Klienten für eine selbstständige Erwerbstätigkeit mit der Vorbereitung auf eine abhängige Erwerbstätigkeit auf eine Stufe stellte. Ziel war die Entwicklung eines kleinen Pilotprojekts, um einen Ansatz zu testen, bevor eine Umsetzung im größeren Stil stattfand. 


\section{Aktivitäten}

Als integriertes Unterstützungspaket bietet das UGP Schulungsmodule, Workshops, Unternehmensberatung durch professionelle Management-Berater und finanzielle Unterstützung zur Deckung der allgemeinen Lebenshaltungskosten während der Frühphase der Geschäftsentwicklung.

Das Programm wurde 1995 als Pilotprojekt in den Bundesländern Steiermark und Kärnten ins Leben gerufen und läuft nach wie vor. Es wird inzwischen in allen neun Bundesländern umgesetzt und wird von den Bundesbüros des AMS in den einzelnen Bundesländern verwaltet. Zu seinen Aktivitäten gehören Beratung, Unternehmerschulungen und finanzielle Unterstützung zur Deckung der allgemeinen Lebenshaltungskosten. Die Unterstützung wird in Zusammenarbeit zwischen dem AMS und externen Management-Beratern geleistet, dieSchulungen und Unternehmensberatungbereitstellen.Seit2000wähltder AMS externe Beratungsdienste für Unternehmensgründungen in einem offenen Ausschreibungsverfahren in der Europäischen Union gemäß nationalem Recht für die Vergabe von Aufträgen aus.

Die Bundesbüros des AMS entscheiden in jedem Bundesland selbst über den Umfang und die Art der Beratungsdienste (d. h. individuelle Beratung, Workshops in Gruppen oder eine Kombination aus beiden). In jedem Bundesland ist ein bestimmter externer Beratungsdienst für die Umsetzung des Unternehmensgründungsprogramms verantwortlich. Die Klienten können sich ihren Anbieter nicht aussuchen, sondern müssen den autorisierten Beratungsdienst in dem Bundesland nutzen, in dem sie leben.

Teilnehmer am UGP müssen beim AMS arbeitslos gemeldet oder Angestellte sein, denen gekündigt wurde und deren Beschäftigung in Kürze beendet sein wird. Außerdem muss eine Bereitschaft vorliegen, ein Unternehmen zu gründen, eine bestimmte Geschäftsidee muss vorhanden sein, und die Person muss einschlägige berufliche Fähigkeiten haben. Einige Arbeitslose, die keine Arbeitslosenunterstützung beziehen, können auch am UGP teilnehmen, zum Beispiel Frauen nach der Elternzeit.

Die im Rahmen des UGP gegründeten Unternehmen müssen neue Unternehmen oder FranchiseUnternehmen von bereits existierenden Unternehmen sein. Freiberufliche Tätigkeiten wie Anwälte, Ärzte oder Apotheker gehören nicht dazu. Die selbstständige Erwerbstätigkeit muss die Hauptbeschäftigung des Teilnehmers sein (AMS, 2014a).

Die Unterstützung durch das UGP ist in vier Phasen aufgebaut:

\section{Klärungsphase}

Das Ziel dieser Phase ist die Abklärung der Realisierbarkeit der Geschäftsidee, die Ermittlung des Schulungsbedarfs der Klienten und die Auswahl derjenigen, die durch das Programm unterstützt werden sollen.

Klienten des AMS, die sich für eine Teilnahme an dem Programm interessieren, müssen ein zweistufiges Screening-Verfahren durchlaufen, bevor sie Unterstützung erhalten. Zunächst müssen sie ihren AMSBerater über ihre Absicht, ins Programm einzusteigen, und über ihre Geschäftsidee informieren. Nach dieser ersten Vorabprüfung der Geschäftsidee durch die regionalen Geschäftsstellen des AMS erhalten die Klienten einen Beratungstermin mit einem externen Geschäftsexperten, der die Realisierbarkeit der Geschäftsidee, die persönliche Eignung des Kandidaten und den persönlichen Qualifizierungsbedarf prüft. Um für eine Teilnahme am Unternehmensgründungsprogramm infrage zu kommen, müssen Bewerber die folgenden Voraussetzungen erfüllen:

- Sie müssen arbeitslos sein;

- sie müssen die Absicht haben, eine selbstständige Erwerbstätigkeit aufzunehmen;

- sie müssen eine konkrete Geschäftsidee haben und über die notwendigen Fähigkeiten und Qualifikationen für eine Unternehmensgründung verfügen;

- sie müssen an einer Gründungsberatung teilnehmen, die vom Arbeitsmarktservice zusammen mit einer externen Beratungsfirma für Start-ups angeboten wird;

- sie müssen zustimmen, eine förmlicheVerpflichtung mit dem Berater des Arbeitsmarktservice einzugehen (d. h. eine Vereinbarung schließen);

- sie müssen einen Nachweis über ihre Sozialversicherung vorlegen (für selbstständig Erwerbstätige oder für Landwirte). 
Diese externe Bewertung bildet die Grundlage für die Entscheidung, ob ein Klient am Unternehmensgründungsprogramm teilnehmen kann oder nicht. Nur diejenigen, bei denen man von einer hohen Wahrscheinlichkeit ausgeht, dass sie ein neues Unternehmen gründen werden, können ins Programm einsteigen (AMS, 2014a).

Die Klärungsphase dauert in der Regel acht bis zehn Wochen. Während der Klärungsphase beziehen Klienten weiterhin ihre Arbeitslosenunterstützung, jedoch wird keine sonstige finanzielle Unterstützung geleistet.

\section{Vorbereitungsphase}

Diese Phase bildet die Einführung in das Programm und beinhaltet unterstützende Beratung bei der Ausarbeitung des Geschäftskonzepts und des Finanzplans und bei der Entwicklung einer Analyse des Qualifikationsbedarfs des Klienten. Dies geschieht vor allem durch persönliche Gespräche, doch werden auch optionale Workshops zu verschiedenen Fragen der Unternehmensgründung angeboten.

Die persönliche Beratung konzentriert sich auf verschiedene Themen im Zusammenhang mit der Unternehmensgründung. Der Schwerpunkt bei der Unterstützung liegt zwar auf der Entwicklung der Geschäftsidee, beinhaltet jedoch auch Marktanalysen, die Beurteilung von Konkurrenten, der Unternehmensstandort, die Organisationsstruktur und rechtliche Voraussetzungen (d. h. Handelsrecht, Arbeitsrecht, Sozialversicherung).

Die Agenturen, die diese Unterstützung bereitstellen, bewerten zudem die Qualifikationen des Klienten und bieten Schulungen und Workshops $\mathrm{zu}$ Unternehmensbuchhaltung, Investitionen und Finanzdienstleistungen an. Sie arbeiten mit potenziellen Kapitalgebern zusammen und informieren die Klienten über ihre Instrumente zur finanziellen Unterstützung, insbesondere Mikrokredite. Mikrokredite werden von UGP-Klienten sehr häufig in Anspruch genommen, weil sie aufgrund ihres Status als Arbeitslose Schwierigkeiten haben, Zugang zu Bankkrediten zu erhalten.

Außerdem werden optionale Workshops zu Marketing, Kostenrechnung, Betriebswirtschaft und den rechtlichen Voraussetzungen für eine Unternehmensgründung angeboten. In den Workshops werden auch persönliche Kompetenzen behandelt und Aspekte wie die Nutzung individueller Stärken und Zeitmanagement angesprochen. Workshops werden in kleinen Gruppen von zehn bis zwölf Teilnehmern angeboten. Jeder Klient kann an bis zu neun Workshops teilnehmen.

Zudem können die Kandidaten an kurzen Einzelschulungskursen teilnehmen. Das Programm stellt bis zu 2600 EUR pro Klient für Kurse bereit, die sich an der Bedarfsanalyse des Klienten orientieren. Am häufigsten werden Kurse in Marketing und Kostenrechnung oder Vorbereitungskurse für Meisterprüfungen angeboten. In der Praxis machen nur wenige Klienten von dieser Option Gebrauch; es kommt häufiger vor, dass Klienten an den UGP-Workshops teilnehmen.

Die Zuwendung, die während der ersten Unterstützungsphase gewährt wird, endet nach zwei Monaten.

\section{Realisierungsphase}

In dieser Phase starten dieTeilnehmer ihr Unternehmen. Eine zusätzliche Unternehmensberatung wird angeboten, und die Klienten erhalten zwei Monate lang eine Zuwendung für die Unternehmensgründung. Die Zuwendung wird rückwirkend ab dem ersten Tag des Monats bezahlt, in dem der Klient sich selbstständig gemacht hat. Diese Zuwendung für die Unternehmensgründung hilft den Klienten, ihre Lebenshaltungskosten zu decken. Sie soll nicht zur Finanzierung von Geschäftstätigkeiten oder für den Erwerb von Betriebsmitteln verwendet werden. Die Zuwendung für die Unternehmensgründung entspricht in der Höhe der Arbeitslosenunterstützung, die der Klient erhalten hat, zuzüglich der von ihm zu zahlenden Sozialversicherungsbeiträge.

Die Vorbereitungs- und Realisierungsphase dauern für die Teilnehmer (zusammen) sechs Monate. Eine Verlängerung auf neun Monate ist jedoch möglich.

\section{Nachbetreuungsphase}

In dieser Phase liegt der Schwerpunkt auf der Überwachung der Geschäftstätigkeit der Teilnehmer durch die Unternehmensberater, damit sichergestellt ist, dass sie nicht auf Schwierigkeiten stoßen. 
Die Klienten können innerhalb von zwei Jahren ab der Unternehmensgründung kostenfrei vier Beratungssitzungen in Anspruch nehmen.

\section{Teilnehmerprofil}

Etwa $40 \%$ der Teilnehmer am UGP sind Frauen. Dieser Anteil lag im Zeitraum zwischen 1998 und 2005 noch bei 30\%. Er entspricht nun dem Anteil der Frauen an der arbeitslos gemeldeten Bevölkerung. Zwischen 2006 und 2012 stieg der Anteil der Älteren (ab 45 Jahren) von 20\% auf 27,5\%, der Anteil der Zuwanderer von 13,8\% auf 16,8\%. Zuwanderer sind in diesem Programm nach wie vor unterrepräsentiert.

Mehr als $40 \%$ der Teilnehmer haben mindestens eine Lehre oder eine Berufsausbildung (42,3\%) abgeschlossen. Die jungen Menschen unter den Teilnehmern (die bis zu 25-Jährigen) haben häufig einen hohen Bildungsstand erreicht - 56,8\% hatten 2012 mindestens eine Berufsausbildung abgeschlossen (Bergmann et al., 2013).

\section{Projektentwicklung}

Seit Programmstart wurden drei Hauptänderungen vorgenommen. Erstens umfasste das Programm anfangs nur die ersten drei Unterstützungsphasen. Die abschließende Nachbetreuungsphase wurde ergänzt, nachdem festgestellt wurde, dass die ersten Kohorten eine zusätzliche Unterstützung nach der Unternehmensgründung benötigten. Zweitens wurden gezielte Angebote entwickelt, um drei spezielle Zielgruppen zu erreichen: Frauen, die wieder eine Erwerbstätigkeit aufnehmen, Ältere und Menschen mit Migrationshintergrund. Drittens wurde die Art der Unternehmensberatung an die sich ändernden Bedürfnisse der Klienten angepasst. In der Frühphase des Programms stützte man sich stärker auf persönliche Treffen und Workshops, doch dieses Vorgehen wich einer Beratung auf Abruf über E-Mail oder Telefon. Auch auf der Bereitstellung von Online-Unterstützung liegt ein starker Fokus, um die Programmkosten zu reduzieren.

Zudem laufen Gespräche darüber, das Programm auf die Bedürfnisse älterer Menschen (d. h. Menschen über 50 Jahre) zuzuschneiden, da diese auf dem Arbeitsmarkt mit Herausforderungen konfrontiert sind. Eine der Überlegungen im Rahmen der Anpassung des Programms an diese Zielgruppe ist, dass die Gruppe in der Tendenz risikoscheuer ist und daher unter Umständen mehr Zeit benötigt, um sich für eine selbstständige Erwerbstätigkeit zu entscheiden. Daher reicht die Dauer des Unterstützungsangebots womöglich nicht aus.

\section{Projektfinanzierung}

Das Unternehmensgründungsprogramm wird durch den Arbeitsmarktservice finanziert. Im Jahr 2013 nahmen 8683 Menschen an dem Programm teil. Die Ausgaben für Beratungsleistungen beliefen sich auf 5 Millionen EUR. Zusätzlich erhielten 5074 neue Existenzgründer im Jahr 2013 Zuwendungen für die Unternehmensgründung in Höhe von 12 Millionen EUR (AMS, 2014b).

Das UGP erhielt bis 2006 Mittel aus dem Europäischen Sozialfonds. Seither finanziert es sich aus nationalen Quellen.

\section{Herausforderungen}

Die größte Herausforderung für das UGP besteht darin, Klienten zu identifizieren und auszuwählen, die in die Klärungsphase einsteigen. Dies ist die wichtigste Phase, da sie über den Erfolg des Programms entscheidet. Die Geschäftsexperten, die die Kandidaten auswählen, müssen zwischen der Notwendigkeit, nachhaltige Start-ups zu entwickeln, und dem Ziel, eine breite Basis von Arbeitslosen zu unterstützen, abwägen. Die Auswahlmethode wurde anhand der gewonnenen Erfahrungen verfeinert, wird aber fortwährend überprüft.

Eine weitere Herausforderung ist die Erhöhung der Kosteneffizienz des Programms. Die Beratungsdienste mussten die besten Verfahren ermitteln, um den Kandidaten unternehmerische Fähigkeiten zu vermitteln. Nach Versuchen mit persönlicher Beratung wechselten die Programmleiter zu einem Ansatz, der persönliche Beratungen mit Workshops kombiniert. Es war eine Verbesserung der Fähigkeiten der Teilnehmer zu bemerken. 


\section{Auswirkungen}

Zwischen 2006 und 2014 gründeten etwa 65000 Arbeitslose im Rahmen dieses Programms ein Unternehmen. Im Jahr 2013 bzw. 2014 gründeten 5074 bzw. 5167 Klienten ein Unternehmen.

Evaluierungen zufolge hilft das Programm erfolgreich Arbeitslosen bei der Unternehmensgründung und der anschließenden Entwicklung eines nachhaltigen und wachsenden Unternehmens. Die Unternehmensgründungsrate (d. h. der Anteil der Teilnehmer, die ein Unternehmen gründeten) lag 2006 bei 75\% und erhöhte sich 2011 auf 83\% (Bergmann et al., 2013).

Die Überlebensraten dieser Unternehmen sind in Tabelle 3.1 dargestellt. Die Überlebensraten der Unternehmen nach einem Jahr und nach drei Jahren sind im letzten Jahrzehnt angestiegen, während die Überlebensrate nach fünf Jahren relativ konstant geblieben ist. Die Überlebensraten der Unternehmen, die durch dieses Programm unterstützt wurden, übersteigen die Überlebensraten der allgemeinen Unternehmerbevölkerung.

\section{Tabelle 3.1. Überlebensraten für Start-ups im Unternehmensgründungsprogramm}

\begin{tabular}{lcc}
\hline & Kohorte 2000 & Kohorte 2009 \\
\hline Ein Jahr & $80 \%$ & $89 \%$ \\
Drei Jahre & $70 \%$ & $75 \%$ \\
Fünf Jahre/immer noch aktiv & $67 \%$ & $64 \%$ \\
\hline
\end{tabular}

Quelle: Bergmann et al., 2013.

Die Evaluierungen zeigen außerdem, dass vom UGP unterstützte Start-ups Beschäftigung für andere schaffen. Einer Evaluierung aus dem Jahr 2006 zufolge schufen unterstützte Start-ups nach fünf Jahren im Durchschnitt netto 1,26 neue Vollzeitstellen. Darin nicht inbegriffen ist der Klient selbst, allerdings sind die Arbeitsplätze berücksichtigt, die wegen Unternehmensaufgabe abgebaut wurden (Dornmayr und Lenger, 2006). Im Rahmen der Evaluierung aus dem Jahr 2013 stellte sich zudem heraus, dass einige der Unternehmen erfolgreich weitere Arbeitsplätze schufen, dass die Mehrheit der Startups jedoch von Einzelunternehmern geführt wurden. Sie zeigte, dass $22 \%$ der jungen Unternehmer, die am UGP teilnahmen, Arbeitgeber waren und dass Frauen weniger häufig als Männer Arbeitgeber waren (15\% gegenüber 28\%). Dieser Umstand wurde auf die Geschäftstätigkeit und Arbeitsintensität zurückgeführt (Bergmann et al. 2013). Nichtsdestotrotz werden die arbeitsmarktpolitischen Ziele des UGP (d. h. die Verringerung der Arbeitslosigkeit durch die Schaffung einer stabilen selbstständigen Erwerbstätigkeit einerseits und die Schaffung neuer Arbeitsplätze in den neuen Unternehmen andererseits) daher weitgehend erreicht.

Die Evaluierungen zeigten auch einige Bereiche auf, in denen das Programm gestärkt werden könnte. Auch wenn laut der 2013 durchgeführten Evaluierung 31\% bzw. 55\% der Teilnehmer ziemlich oder sehr zufrieden mit der Beratung im Rahmen des Programms waren, wird in der Evaluierung empfohlen, während der bereitgestellten Beratungen mehr Gewicht auf eine sektorspezifische Beratung zu legen (Bergmann et al. 2013). Außerdem wurde vorgeschlagen, dass das Programm mehr Möglichkeiten für Netzwerkaktivitäten für die Teilnehmer anbieten und die Klienten besser über die nach der Unternehmensgründung zur Verfügung stehende Unterstützung informieren sollte.

\section{Voraussetzungen für die Übertragung}

Das Unternehmensgründungsprogramm (UGP) hat das Potenzial, auf andere Länder und Regionen übertragen zu werden, und kann von Erfolg gekrönt sein, wenn es auf den lokalen Kontext zugeschnitten wird. Die zentralen Erfolgsfaktoren sind folgende:

1. Finanzielle Unterstützung. Einer der zentralen Erfolgsfaktoren für dieses Programm ist die finanzielle Unterstützung zur Deckung der persönlichen Ausgaben während des Gründungsprozesses. Sie ist besonders relevant für diese Klientengruppe (d. h. Arbeitslose), die wahrscheinlich nur über wenige Ersparnisse verfügt, auf die sie in der Frühphase der Geschäftsentwicklung zurückgreifen kann. Es ist wichtig, Zuwendungen bereitzustellen, mit denen die grundlegenden Lebenshaltungskosten 
und Sozialversicherungsbeiträge gedeckt werden können. Die finanzielle Unterstützung sollte nur für einen begrenzten Zeitraum geleistet werden, um keine Abhängigkeit zu schaffen.

2. Niemand sollte zur Teilnahme am Programm gedrängt werden. Die Berater in den regionalen Geschäftsstellen des AMS schlagen ihren Klienten keine selbstständige Erwerbstätigkeit vor. Die Klienten werden über diese Möglichkeit informiert, aber die Entwicklung einer eigenen Geschäftsidee ist ihnen selbst überlassen.

3. Es sollten Teilnehmer mit Potenzial ausgewählt werden. Die Evaluierung aus dem Jahr 2013 empfiehlt einen noch stärkeren Fokus auf die Auswahl von Start-ups, die das Potenzial besitzen, Gewinne zu erwirtschaften, da viele Teilnehmer mit der Ertragslage ihres neuen Unternehmens nicht zufrieden sind. Nur 36\% der männlichen und 26\% der weiblichen Gründer gaben an, die Einnahmen aus ihrer selbstständigen Erwerbstätigkeit seien ausreichend.

\section{Textfeld 3.1. Übertragung des österreichischen Unternehmensgründungsprogramms auf Rumänien}

Einer der beauftragten externen Beratungsdienste, die ÖsB Consulting $\mathrm{GmbH}$, machte im Rahmen des Projekts „Schaffe deinen eigenen Arbeitsplatz“, das 2010 mit Mitteln aus dem Europäischen Sozialfonds umgesetzt wurde, Erfahrungen mit der Übertragung des Programms auf den ländlichen Raum in Rumänien. Während des drei Jahre laufenden Projekts passten sie das Unternehmensgründungsprogramm an die lokalen Bedürfnisse, Einrichtungen und wirtschaftlichen Bedingungen an. Eine der größten Herausforderung bestand darin, qualifizierte Berater im ländlichen Raum zu finden, die die Unterstützung leisten würden. Einer der wesentlichen Unterschiede zwischen dem in Rumänien und dem in Österreich durchgeführten Programm ist, dass in Rumänien ein Kurs stattfand, um auf strukturiertere Weise unternehmerische Fähigkeiten zu vermitteln, da die Klienten dort weiter verstreut sind als in Österreich.

\section{Literatur}

Arbeitsmarktservice Österreich (2014a), Bundesrichtlinie Unternehmensgründungsprogramm für Arbeitslose (UGP), Wien: Arbeitsmarktservice Österreich.

Arbeitsmarktservice Österreich (2014b), Geschäftsbericht 2013: Und trotzdem: 2013 war ein schwieriges Jahr am österreichischen Arbeitsmarkt, Wien: Arbeitsmarktservice Österreich.

Bergmann, N., A. Riesenfelder, S. Schmatz und C. Sorger (2013), Evaluierung des Unternehmensgründungsprogramms (UGP), Wien: L\&R Sozialforschung/AMS Österreich.

Dornmayr, H. und B. Lenger (2006), Evaluierung der Maßnahmen der GründerInnenförderung des AMS. Wien: Institut für Bildungsforschung (ibw)/AMS Österreich. 


\section{DreamStart, Belgien (Brüssel)}

In dieser Fallstudie wird ein Programm vorgestellt, das arbeitslose junge Menschen durch Schulungen, Coaching und Mentoring bei der Unternehmensgründung unterstützt. Die Fallstudie legt die Ziele und den Hintergrund des Programms dar und beschreibt seine zentralen Aktivitäten. Weiterhin beschreibt sie die größten Herausforderungen bei der Gestaltung und Umsetzung des Programms, die erzielten Auswirkungen und die Voraussetzungen für die Übertragung dieses Programms auf einen anderen Kontext.

\section{Ziele}

Bei DreamStart handelt es sich um ein integriertes Unternehmensgründungsprogramm, dessen Ziel die Unterstützung arbeitsloser junger Menschen (unter 30 Jahre) bei der Unternehmensgründung im Raum Brüssel ist. Das 2013 ins Leben gerufene Programm ist auf die Bedürfnisse und Erwartungen junger Menschen ausgerichtet und dauert weiterhin an.

Auf der Grundlage des Programms Créajeunes, das von Adie in Frankreich entwickelt wurde, begegnet DreamStart den Herausforderungen, mit denen junge Menschen beim Erwerb unternehmerischer Fähigkeiten und beim Zugang zu Finanzierung konfrontiert sind. Es fördert den Erwerb unternehmerischer Fähigkeiten durch formelle Unternehmerschulungen vor der Unternehmensgründung sowie durch informelle Methoden wie Coaching und Mentoring. Freiwillige sowie erfahrene Geschäftsexperten aus Organisationen des öffentlichen und privaten Sektors spielen eine wesentliche Rolle bei der Bereitstellung dieser Dienste. Der Zugang zu Finanzierung wird durch Mikrofinanzierungsinstitutionen ermöglicht, am häufigsten durch MicroStart, eine der wachstumsstärksten Mikrofinanzierungsinstitutionen in Belgien.

\section{Hintergrund}

Im Raum Brüssel herrscht eine hohe Jugendarbeitslosigkeit. Im Januar 2013 war fast einer von drei Arbeitslosen im Raum Brüssel unter 30 Jahre alt. Die hohe Jugendarbeitslosigkeit stellt eine Herausforderung für die öffentlichen Arbeitsverwaltungen dar, denen es zunehmend schwerfällt, eine Beschäftigung für die jungen Menschen zu finden. Die selbstständige Erwerbstätigkeit gilt als eine potenzielle (Teil-)Lösung für dieses Problem.

Die Jugendarbeitslosigkeit muss dringend angegangen werden, denn lange Zeiten der Arbeitslosigkeit haben für junge Menschen langfristige Konsequenzen (sogenannte „Narbeneffekte“). So ist bei ihnen zum Beispiel die Wahrscheinlichkeit größer, als erwachsener Mensch arbeitslos zu sein und im Laufe ihres Lebens ein kleineres Einkommen zu haben. 


\section{Aktivitäten}

DreamStart bietet eine zweimonatige Unterstützung, darunter Schulungen vor der Unternehmensgründung sowie Coaching und Mentoring, um arbeitslose junge Menschen bei der Entwicklung einer Geschäftsidee und der Erstellung eines Geschäftsplans zu helfen.

\section{Kontaktaufnahme und Bereitstellung von Informationen}

Alle der wichtigsten Interessenträger von DreamStart werben für das Projekt. Dazu gehören die Organisation, die das Projekt durchführt (MicroStart), sowie die lokale Unternehmensagentur im Raum Brüssel. Die Werbung erfolgt verbal durch Präsentationen und Treffen sowie durch Drucksachen, die in den Büros der Unternehmensagentur ausliegen. Die Kontaktarbeit wird vor jedem neuen Zyklus verstärkt.

\section{Auswahl der Teilnehmer}

Der primäre Aufnahmemechanismus besteht bei DreamStart aus Einzelgesprächen zwischen Projektmanager und potenziellem Klienten. Die Projektmanager wählen die Teilnehmer anhand von drei Hauptkriterien aus:

- ihre Einstellung zur selbstständigen Erwerbstätigkeit und ihr Wunsch, ein Unternehmen zu gründen;

- die Tragfähigkeit ihrer Geschäftsidee und die erwarteten Resultate des Produkts bzw. der Leistung, das bzw. die vorgeschlagen wird;

- die Übereinstimmung zwischen der Geschäftsidee und dem beruflichen und technischen Fachwissen des Teilnehmers sowie seiner Erfahrung in diesem Bereich.

\section{Schulungen und Coaching}

In den von DreamStart durchgeführten Schulungen werden die folgenden vier Themenbereiche behandelt: Entwicklung des Unternehmertums (36 Stunden); Marketing und Entwicklung eines Vermarktungsplans (24 Stunden); Geschäftsplanung (18 Stunden) und Finanzplanung (48 Stunden) (siehe Abbildung 4.1). Dieser Ansatz baut auf dem Business Model Canvas, das alle Elemente eines Geschäftsund Marketingplans in einem Plan vereinigt, sowie dem Modell Clé de Réussite, das sich auf Finanzplanung konzentriert. Die Gesamtdauer der Unterstützung beträgt zwei Monate mit etwa 126 Schulungsstunden. Diese werden in einer Mischung aus Frontalunterricht, Gruppenarbeit und Einzelaufgaben durchgeführt.

\section{Abbildung 4.1. Die Bestandteile der Schulungen von DreamStart}

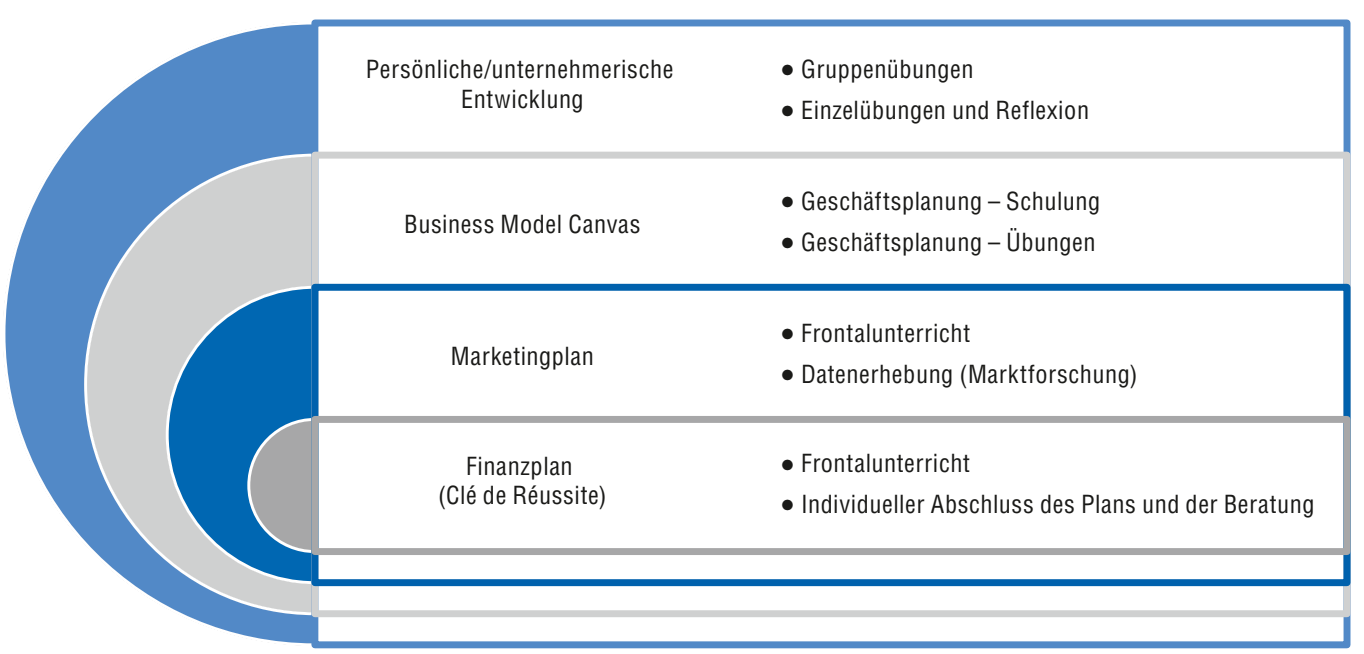

Dieser vielseitige Schulungsansatz ermöglicht dem Programm, gleichzeitig die persönliche Entwicklung als auch die Unternehmensgründung anzugehen. Dies ist beim Aufbau von Selbstvertrauen von Bedeutung und verringert die Gefahr einer sozialen Ausgrenzung. Zudem ist die Bereitstellung 
der Schulungen durch Fachkräfte aus dem privaten und dem öffentlichen Sektor praxisorientiert und zielführend. Parallel zu den Schulungen kann jeder Teilnehmer ein individuelles Coaching und Mentoring durch die Ausbilder in Anspruch nehmen.

Offiziell endet die Unterstützung, sobald die Teilnehmer ihren Geschäftsplan entwickelt und dazu eine Rückmeldung von einem externen Bewertungsgremium erhalten haben. Allerdings ist es durchaus üblich, dass die Teilnehmer informelle Unterstützungsgruppen bilden, in denen sie sich gegenseitig helfen und beraten. Diese informelle Netzwerkarbeit würden die Mitarbeiter von DreamStart künftig gern in formellerer Weise in das Programm integrieren.

\section{Mikrokredite}

Mikrokredite sind nicht direkt Teil von DreamStart; den Teilnehmern wird es freigestellt, sich bei beliebigen Finanzinstituten oder Banken um eine externe Finanzierung zu bemühen. Jedoch bietet MicroStart in der Praxis häufig denjenigen Mikrokredite an, die das Programm abgeschlossen haben. Im Durchschnitt liegt die Summe der pro Teilnehmer gewährten Kredite zwischen 15000 EUR und 25000 EUR. Diese Summe liegt dabei höher als die des üblicherweise durch MicroStart bereitgestellten Darlehens.

\section{Projektpartner}

MicroStart ist der Projektmanager. Die Organisation hat die Unterstützung entworfen und kofinanziert die Tätigkeiten zusammen mit privatwirtschaftlichen Spenden durch MicroStart Support vzw, einer Schwesterorganisation, die als gemeinnützige Organisation eingetragen ist und somit laut Gesetz solche Spenden erhalten darf.

MicroStart wurde von Adie (Frankreich) und BNP Paribas Fortis gegründet und besteht aus zwei Einheiten, die jeweils rechtlich als unabhängige Sozialunternehmen eingetragen sind: MicroStart Support vzw verwaltet die Schulungen und das Coaching, während MicroStart cvba sich um die Mikrokredite kümmert. MicroStart erhält finanzielle Unterstützung von der Europäischen Kommission im Rahmen des Progress-Mikrofinanzierungsinstruments.

Bei MicroStart sind zwei engagierte Mitarbeiter tätig, die das Programm organisieren, weiterentwickeln und umsetzen. Diese beiden erfahrenden Unternehmensberater verwalten das Programm, sind als Ausbilder tätig und überwachen und führen die Freiwilligen im Programm.

Ein besonderes Merkmal des DreamStart-Projekts ist die Mitwirkung von Freiwilligen als Ausbilder und Betreuer. Bei den meisten handelt es sich um erfahrene Geschäftsexperten, die als Unternehmensberater oder Fachkräfte im Finanzsektor (z. B. Wirtschaftsanalytiker, Kreditberater, Wirtschaftsprüfer) arbeiten, während andere Fachkräfte im Ruhestand sind. Die Freiwilligen wenden sich aktiv an MicroStart, um sich an den Aktivitäten zu beteiligen. Einige werden an dieses Programm verwiesen. Alle Freiwilligen absolvieren einen speziellen Schulungskurs, der Folgendes umfasst:

- einen Einführungskurs, in dem sie eine allgemeine Orientierung und Informationen über DreamStart erhalten;

- eine Schulung, wie sie die Unterstützung und die Schulungsmodule bereitstellen sollten; und

- Mentoring und praktische Beratung während eines Zyklus.

Nach der Mitwirkung an einem Zyklus wird von den Freiwilligen erwartet, dass sie im Programm selbstständig arbeiten und sich an die festgelegte Struktur halten. Die Freiwilligen arbeiten mit den Koordinatoren von DreamStart zusammen, um sicherzustellen, dass das Programm wie beabsichtigt durchgeführtwird.Anderean den Schulungen beteiligtePartnersind Group One (eineSchulungsorganisation), BECI (die Handelskammer) und impulse.brussels (eine Unternehmensagentur in Brüssel).

\section{Projektentwicklung}

Die einzige Veränderung, die seit dem Start des Programms vorgenommen wurde, war eine Anpassung des Aufnahmeverfahrens. Anfangs setzte DreamStart in hohem Maße auf Weitervermittlungen von Partnerorganisationen. Dies verringerte zwar die Notwendigkeit einer umfangreichen Kontaktarbeit, hatte jedoch eine sehr einheitliche Teilnehmergruppe zur Folge und 
beschränkte die Reichweite des Programms. Von dieser Methode nahm man Abstand. Nun betreibt das Programm umfassende Eigenwerbung, um seine Zielklienten besser zu erreichen.

\section{Projektfinanzierung}

Die jährlichen Kosten von DreamStart, einschließlich Organisation, Verwaltung und Schulungskursen, werden auf rund 72000 EUR geschätzt. Die Ausgaben bilden die Löhne der Projektträger und -betreuer (60000 EUR), die Kosten der Schulungseinrichtungen (5000 EUR) sowie Werbung und sonstige Kosten (5000 EUR). Durch den Einsatz freiwilliger Betreuer und Ausbilder werden Einsparungen von etwa 70000 EUR jährlich erzielt (ausgehend von geschätzten täglichen Kosten von 1000 EUR pro Freiwilligen).

Diese Kosten decken drei Programmzyklen pro Jahr; die Kosten für jeden Zyklus liegen bei 24000 EUR. An jedem Zyklus sind zwölf Teilnehmer beteiligt; die Kosten pro Teilnehmer betragen 2000 EUR.

Das Programm greift auf drei Hauptfinanzierungsquellen zurück. Öffentliche Mittel decken rund $42 \%$ der Betriebskosten und stammen vom Bundesministerium für soziale Integration und von der Region Brüssel-Hauptstadt. Privatwirtschaftliche Mittel gehen durch Maßnahmen im Bereich der sozialen Verantwortung ein und machen etwa 7\% der Betriebskosten aus. Wesentliche Beiträge erhält das Programm von der BNP Paribas Fortis Foundation und der Levi Strauss Foundation. Den größten Betrag machen Sacheinlagen von Freiwilligen aus, die schätzungsweise knapp 50\% der Betriebskosten decken.

\section{Herausforderungen}

Bei seinen Bemühungen, seine Aktivitäten auszuweiten, ist DreamStart derzeit mit verschiedenen Herausforderungen konfrontiert. Erstens setzt das Programm in sehr hohem Maße auf den Einsatz von Freiwilligen, die die Schulungen und das Coaching durchführen, sodass die Betriebskosten minimiert werden können. Jede Ausweitung der Größenordnung oder des Umfangs des Programms erhöht auch den Bedarf an Freiwilligen. Dies stellt eine Herausforderung dar, da es immer schwieriger wird, sich die Mitwirkung von Freiwilligen zu sichern.

Eine damit zusammenhängende Herausforderung für DreamStart besteht darin, einen Preis für den Dienst festzulegen, der den Teilnehmern bereitgestellt wird. Die Unterstützung wird derzeit für die Teilnehmer kostenlos geleistet. So wird sichergestellt, dass sie für arbeitslose junge Menschen zugänglich ist. Das Programm läuft stets Gefahr, öffentliche oder private Mittel oder Freiwillige zu verlieren. Außerdem wäre für eine Ausweitung der Größenordnung und/oder des Umfangs des Programms eine größere Anzahl von Freiwilligen nötig. Dies kann unter Umständen nicht erreicht werden, da es schwierig ist, Freiwillige zu gewinnen und zu behalten. Daher zieht das Programm in Erwägung, eine kleine Gebühr für die Teilnahme zu erheben, um damit einen Teil der Betriebskosten zu decken. Allerdings würde dies wahrscheinlich einige Menschen von einer Teilnahme abschrecken.

Das Programm konzentriert seine Bemühungen auf die Phase vor der Unternehmensgründung. Jedoch benötigen viele Klienten wahrscheinlich auch nach ihrer Unternehmensgründung weiterhin Unterstützung. Deshalb sucht das Programm nach einer Möglichkeit, sich besser in das Netzwerk von Wirtschaftsförderungsdiensten $\mathrm{zu}$ integrieren, damit die Teilnehmer Zugang zu weiteren Unterstützungsleistungen erhalten. $\mathrm{Zu}$ den weiteren Überlegungen bezüglich einer Ausweitung des Programmumfangs gehören die Bereitstellung eines intensiveren Coachings und Mentorings, um die Teilnehmer durch den Gründungsprozess und darüber hinaus zu führen.

In ähnlicher Weise stellt der Zugang zu Finanzierung für einige Teilnehmer eine Herausforderung dar. DreamStart kann den Zugang zu Mikrokrediten von MicroStart für einige Teilnehmer erleichtern, aber MicroStart ist eine unabhängige Organisation, die nicht alle der Klienten von DreamStart akzeptiert. DreamStart versucht daher, Partnerschaften mit anderen Finanzeinrichtungen aufzubauen, um den Teilnehmern mehr Finanzierungsoptionen und einen Zugang zu spezialisierteren Produkten zu bieten, zum Beispiel Versicherungen für diejenigen, die Schwierigkeiten beim Zugang zu allgemeinen Produkten und Dienstleistungen haben.

Und schließlich strebt DreamStart an, eine aktivere Rolle in politischen Dialogen einzunehmen, um politische Entscheidungsträger über die Herausforderungen zu informieren, mit denen DreamStarts Klienten konfrontiert sind. Zum Beispiel möchten einige Teilnehmer ihr Start-up mit anderen Aktivitäten 
kombinieren (d. h. als „hybride Unternehmer“), laufen aber das Risiko, ihre Sozialhilfeleistungen zu verlieren, weil sie unter Umständen nicht als für eine Beschäftigung verfügbar betrachtet werden.

\section{Auswirkungen}

Seit Anfang 2013 hat DreamStart vier Zyklen dieses Projekts durchgeführt. Jeder Zyklus wurde erfolgreich von zehn bis zwölf Teilnehmern abgeschlossen. Etwa die Hälfte der jungen Menschen, die an den Aufnahmegesprächen teilnehmen, werden angenommen, und die Ausstiegsquote liegt bei zwei pro Zyklus.

Rund $60 \%$ der jungen Menschen, die das Programm abschließen und dem Gremium ihren Geschäftsplan vorlegen, gründen innerhalb eines Jahres ein Unternehmen. Überwachungen zufolge gründet ein Drittel der Teilnehmer, die einen Zyklus abgeschlossen haben, im Anschluss ein Unternehmen und geht dieser Tätigkeit in Vollzeit nach. Zwei Drittel arbeiten in Teilzeit und kombinieren ihre selbstständige Erwerbstätigkeit mit einer abhängigen Erwerbstätigkeit. Dadurch können sie ihr gegründetes Unternehmen mit den Einnahmen aus der abhängigen Erwerbstätigkeit finanzieren.

Als unerwartetes Resultat schuf das Programm Netzwerke von Jungunternehmern. Die Teilnehmer bleiben in der Regel nach den Schulungen in Kontakt miteinander und unterstützen sich als Gleichgestellte gegenseitig.

\section{Voraussetzungen für die Übertragung}

Bei der Anpassung dieses Programms an einen anderen Kontext kann die umsetzende Organisation ihre Erfolgsaussichten verbessern, wenn folgende Voraussetzungen erfüllt sind:

1. Sicherstellung eines adäquaten Angebots an freiwilligen Geschäftsexperten. Die Nachbildung dieses Programms an einem anderen Standort erfordert Zugang zu freiwilligen Geschäftsexperten, die bereit sind, ihr Wissen und ihre Expertise zu teilen. Dazu ist wahrscheinlich ein Projektmanager notwendig, der in der Wirtschaftsgemeinschaft bekannt und geachtet ist und über ein großes, entwickeltes Netzwerk verfügt, das für die Suche nach Freiwilligen genutzt werden kann.

2. Bereitstellung von Schulungen für Freiwillige. Die umsetzende Organisation muss in der Lage sein, die Freiwilligen zu schulen und anzuleiten. Dies geht über die Erläuterung der Verfahren hinaus und beinhaltet Kommunikations- und Coaching-Schulungen, damit die Freiwilligen ihr Potenzial als Unterstützungsgeber ausschöpfen können.

3. Sicherung einer ausreichenden Finanzierung. Für eine Übertragung des Programms ist eine Finanzierung notwendig. Wahrscheinlich müssen mehrere Quellen herangezogen werden, wie das auch bei DreamStart der Fall ist. Die Finanzierung wird zur Entwicklung der Schulungskomponente und die entsprechende Werbung, für die Schulung und Unterstützung von Freiwilligen, zur Sicherung eines physischen Standorts für die Schulungen und zur Deckung des Gehalts des Projektmanagers benötigt.

4. Vorhandensein eines physischen Standorts. Die umsetzende Organisation muss über Einrichtungen verfügen, wo sie die Schulungen der Jungunternehmer durchführen kann. Für die Schulungen werden zwar nur ein kleiner Raum und ein Computer benötigt, doch der Standort des Schulungszentrums will gut überlegt sein. Es ist wichtig, dass die jungen Menschen das Schulungszentrum mit den öffentlichen Verkehrsmitteln erreichen können und - bei einer Durchführung des Programms in größerem Umfang (d. h. national) - dass in allen Regionen Schulungszentren eingerichtet werden.

5. Partnerschaften mit unterstützenden Interessenträgern. Partnerschaften sind wichtig für die erfolgreiche Übertragung der Erfahrung auf einen anderen Kontext. Die Zusammenarbeit mit anderen Interessenträgern verbessert die Werbung und die Kontaktarbeit, was in den ersten Projektzyklen insbesondere von Bedeutung ist. Partnerschaften können sich außerdem bei der Sicherung der Mitwirkung von freiwilligen Fachkräften als wertvoll erweisen.

6. Einstellung eines sachkundigen Projektmanagers. Für DreamStart ist es wichtig, einen starken Projektmanager zu haben, der über Fachwissen im Bereich Unternehmertum, Mittelbeschaffung und Sicherung der Mitwirkung Freiwilliger verfügt. Zudem ist ein kleiner Stab von Mitarbeitern wichtig, 
die in der Geschäftsplanung und in der Schulung von Unternehmern Wissen und Erfahrungen mitbringen und bei der Vorbereitung der Schulungen helfen können.

7. Gewährleistung der Glaubwürdigkeit der umsetzenden Organisation. Um potenzielle Projektteilnehmer erfolgreich zu erreichen, sollte die umsetzende Organisation bereits mit dieser Klientengruppe gearbeitet haben, damit sie von den Klienten und Unterstützern des Programms als glaubwürdiger Unterstützungsträger betrachtet wird. Ihre Mitarbeiter müssen Fachkräfte sein, die in der Lage sind, Aufnahmegespräche zu leiten und Schulungen zu organisieren.

8. Aufbau eines Netzwerks aus ergänzenden Organisationen. Eine starke umsetzende Organisation ist zwar wichtig, aber es ist auch von Bedeutung, dass diese von einem Netzwerk aus anderen Unterstützungsträgern umgeben ist, die Lücken im Angebot des Programms schließen können. Dazu zählen zum Beispiel Mikrofinanzierungsinstitutionen, die den Teilnehmern Startkapital und, als Hilfe im Anschluss an die Unternehmensgründung, weitere Wirtschaftsförderungsdienstleistungen bieten können.

\section{Literatur}

MicroStart (2014), Website von MicroStart, http://microstart.be/fr. 


\section{Unternehmertum funktioniert, Belgien (Flandern)}

In dieser Fallstudie wird ein einjähriges Coaching-Projekt zur Unterstützung von Arbeitslosen bei der Unternehmensgründung vorgestellt. Die Beschreibung enthält die Ziele, den Hintergrund und die Aktivitäten des Projekts. Außerdem werden die Herausforderungen bei der Umsetzung des Programms sowie die Auswirkungen des Programms vorgestellt. Auch die Voraussetzungen für die Übertragung dieses Verfahrens auf einen anderen Kontext werden erörtert.

\section{Ziele}

Unternehmertum funktioniert („Ondernemen Werkt") war ein einjähriges Coaching-Programm, das von 2008 bis 2013 in Flandern, einer niederländischsprachigen Region Belgiens, durchgeführt wurde. Sein Ziel war die Unterstützung von arbeitslosen Arbeitsuchenden bei der Gründung eines eigenen Unternehmens. Das Coaching kombinierte Unternehmensberatung, persönliche Entwicklung und die Stärkung der unternehmerischen Fähigkeiten. Im Vergleich zu Gruppenschulungskursen ermöglichte dieser individualisierte Ansatz eine bessere Betreuung während der Phase vor der Unternehmensgründung und bedarfsgerechtere Weitervermittlungen an andere Unterstützungsanbieter (z. B. Geschäftsexperten, Mikrokredite).

Das Projekt zielte darauf ab, über einen Zeitraum von vier Jahren hinweg 650 potenzielle Teilnehmer jährlich zu überprüfen und zu erreichen, dass 25\% dieser Menschen ein Unternehmen gründeten.

\section{Hintergrund}

In Flandern fanden sehr wenige arbeitslose Arbeitsuchende durch eine selbstständige Erwerbstätigkeit zurück ins Arbeitsleben. Daher wurde das Projekt ins Leben gerufen, um zwei Hindernisse anzugehen, vor denen Arbeitslose in Bezug auf die Unternehmensgründung häufig standen. Erstens mangelt es Arbeitslosen im Allgemeinen an Motivation und Fähigkeiten für die selbstständige Erwerbstätigkeit. Zweitens wurden die Arbeitsvermittlungsdienste für Arbeitslose in Flandern Mitte der 2000er-Jahre von mehreren, unabhängig agierenden Akteuren bereitgestellt. Dieses fragmentierte System bot wenig Unterstützung für Arbeitslose, die sich für eine selbstständige Erwerbstätigkeit interessierten.

\section{Aktivitäten}

\section{Erstes Screening}

Ein erstes Screening der interessierten potenziellen Unternehmer wurde durch die VDAB unter Einsatz von Geschäftsexperten (d. h. externe Berater) durchgeführt, die im Bereich selbstständige Erwerbstätigkeit 
spezialisiert waren. Potenzielle Teilnehmer wurden zu einem Gespräch eingeladen, bei dem ihre unternehmerischen Fähigkeiten, Geschäftsidee und Motivation bewertet wurden. Die Geschäftsexperten nutzten ein Instrument namens „Entre Mirror“, einen Fragebogen zur Selbstbewertung, bei dem die Befragten Punkte für unternehmerische Fähigkeiten wie Entschlussfreudigkeit, Unabhängigkeit, Überzeugungskraft, Ausdauer, Finanzwissen, Netzwerkarbeit und organisatorische Fähigkeiten erhalten. Außerdem wurde das Screening-Gespräch genutzt, um die Geschäftsidee zu bewerten, denn das Programm stand nur arbeitslosen Arbeitsuchenden mit einer konkreten, realisierbaren Geschäftsidee offen, die innerhalb eines Jahres umgesetzt werden könnte. Die Kandidaten mussten auch über die notwendigen fachlichen und berufsspezifischen Kenntnisse und das erforderliche Startkapital verfügen.

Auf der Grundlage dieses Screening-Gesprächs sprach die VDAB eine von drei möglichen Empfehlungen aus: Die Kandidaten, die das Screening bestanden, wurden zur Teilnahme an „Unternehmertum funktioniert“ an UNIZO weitergeleitet. Die Kandidaten, die das Screening bestanden, aber eine intensivere Unterstützung zu benötigen schienen, wurden an die Flemish Activity Co-operative (d. h. ein Sozialunternehmen) verwiesen, um dort vor dem Einstieg bei „Unternehmertum funktioniert“ eine Schulung zu absolvieren. Bei denjenigen schließlich, die das Screening nicht bestanden, wurde eine Zulassung zum Programm abgelehnt.

\section{Phase 1: Sondierungsphase}

Die erste Phase dauerte maximal sechs Monate und beinhaltete eine ausführliche Aufnahmeanalyse und Orientierung, um die jeweiligen unternehmerischen Fähigkeiten und den Schulungsbedarf zu ermitteln. Auf der Grundlage dieser Analyse wurde dem Bewerber ein Betreuer zur Seite gestellt, um einen persönlichen Entwicklungsplan und einen Aktionsplan zu entwickeln. So sollte die Realisierbarkeit der Unternehmensgründung innerhalb des durch das Projekt vorgesehenen Zeitrahmens gewährleistet werden.

Während der Sondierungsphase mussten die Teilnehmer die erforderlichen Schritte ergreifen, um ein betriebswirtschaftliches Zertifikat zu erwerben - dies ist in Flandern zwingende Voraussetzung für die Unternehmensgründung. Dazu mussten die Teilnehmer einen (aus 132 Stunden bestehenden) Kurs absolvieren, in dem eine breite Palette an Themen behandelt wurde, die ein Unternehmer zur Führung eines eigenen Unternehmens beherrschen muss. Ein wichtiger Schwerpunkt lag auf der Entwicklung eines Geschäftsplans. Der Kurs dauerte bis zum Abschluss drei Monate, und Teilnehmer mit geringen Fähigkeiten konnten einen 40-stündigen Vorbereitungskurs absolvieren. Nichtmuttersprachler wurden von einem Sprachtrainer betreut.

Ziel dieser Phase war die Weiterentwicklung unternehmerischer Kompetenzen und die Sondierung der Realisierbarkeit der Geschäftsidee. Am Ende dieser Phase entschieden die Teilnehmer, ob sie zur zweiten Phase von „Unternehmertum funktioniert“ übergehen oder aus dem Programm aussteigen wollten, um eine Beschäftigung auf dem Arbeitsmarkt zu suchen.

\section{Phase 2: Vorbereitungsphase}

Diese Phase mit einer Dauer von höchstens sechs Monaten beinhaltete eine intensive Betreuung mit dem Ziel der eigentlichen Einrichtung der selbstständigen Erwerbstätigkeit. In der Vorbereitungsphase erhielt der Unternehmer ein individuelles Coaching und konnte zudem an Gruppen-Workshops teilnehmen. Ziel dieser Phase war die Umsetzung des Plans zum Fähigkeitenerwerb, der während der Sondierungsphase entwickelt wurde und sich auf praktische Aufgaben konzentrierte, zum Beispiel die Beantragung eines Darlehens, die Suche nach Gewerberäumen, die Kontaktaufnahme mit Lieferanten und potenziellen Kunden und die Vorbereitung einer Marktstrategie. Außerdem wurden den Kandidaten Workshops und Informationssitzungen angeboten, aus denen sie wählen konnten, um mehr über Fallstricke für Start-ups und Möglichkeiten zu erfahren, um diese möglichst gering zu halten.

\section{Projektpartner}

Bei „Unternehmertum funktioniert“ handelte es sich um eine Partnerschaft zwischen UNIZO (der Union selbstständig Erwerbstätiger und KMU), der VDAB (der flämischen öffentlichen Arbeitsverwaltung) und Syntra (dem Netzwerk für Unternehmerausbildung). UNIZO stellte ein Team aus fünf Betreuern zur 
Verfügung, die ein Jahr lang ein kostenfreies Coaching für Arbeitslose durchführten, die sich für eine selbstständige Erwerbstätigkeit interessierten (Unternehmerkandidaten). Diese Betreuer waren in den regionalen Geschäftsstellen in jeder der fünf flämischen Provinzen tätig.

Die Geschäftsstellen der VDAB sind über ganz Flandern verteilt; alle arbeitslosen Arbeitsuchenden sind bei der VDAB gemeldet. Die VDAB hat 40 Berater für den Bereich selbstständige Erwerbstätigkeit, die die Unternehmerkandidaten überprüften, die sich für eine Teilnahme an dem Programm interessierten.

Es bestand eine intensive Zusammenarbeit mit Syntra Flandern und vier regionalen Geschäftsstellen von Syntra, um die Kompetenzen der Unternehmerkandidaten zu stärken. Unter anderem bietet Syntra den Kurs „Betriebswirtschaft“ an, der für eine Unternehmensgründung in Flandern zwingend absolviert werden muss.

\section{Teilnehmerprofil}

Tabelle 5.1 stellt Verwaltungsdaten dar, die über die Teilnehmer erhoben wurden, die in die Sondierungsphase des Projekts einstiegen. Die Tabelle zeigt eine Reihe soziodemografischer Merkmale der Teilnehmer. Zum Vergleich sind in der Tabelle außerdem Daten zu den arbeitslosen Arbeitsuchenden und selbstständig erwerbstätigen Unternehmern in Flandern enthalten. Auf diese Weise kann nicht nur geprüft werden, welche Art von Teilnehmern das Programm anzog, sondern auch untersucht werden, inwiefern die Teilnehmer typische arbeitslose Arbeitsuchende oder selbstständig Erwerbstätige repräsentieren.

\section{Tabelle 5.1. Profil der Teilnehmer an „Unternehmertum funktioniert“, 2009-2014} Anteil an der Gesamtanzahl

\begin{tabular}{|c|c|c|c|c|}
\hline \multirow[t]{2}{*}{ Merkmale } & \multicolumn{2}{|c|}{ Unternehmertum funktioniert } & \multirow{2}{*}{$\begin{array}{c}\text { Arbeitslose } \\
\text { Arbeitsuchende } \\
\text { VDAB-Bevölkerung }\left({ }^{1}\right)\end{array}$} & \multirow{2}{*}{$\begin{array}{l}\text { Flämische Bevölkerung } \\
\text { selbstständig erwerb- } \\
\text { stätiger Unternehmer }\left({ }^{2}\right)\end{array}$} \\
\hline & $\begin{array}{c}\text { (Phase 1) } \\
A=2589\end{array}$ & $\begin{array}{c}\text { (Phase 2) } \\
A=534\end{array}$ & & \\
\hline Weiblich & $39,4 \%$ & $43,6 \%$ & $47,4 \%$ & $33,7 \%$ \\
\hline Ab 50 Jahren & $7,3 \%$ & $6,7 \%$ & $25,0 \%$ & $34,8 \%$ \\
\hline Kein Sekundarschulabschluss & $33,3 \%$ & $27,0 \%$ & $49,1 \%$ & - \\
\hline Sekundarschulabschluss & $39,9 \%$ & $38,7 \%$ & $34,7 \%$ & - \\
\hline Bachelor- oder Master-Abschluss & $26,8 \%$ & $34,3 \%$ & $16,2 \%$ & - \\
\hline Erwerbsunfähige & $6,3 \%$ & $4,1 \%$ & $13,9 \%$ & $4,6 \%$ \\
\hline Ethnische Minderheiten & $18,7 \%$ & $10,6 \%$ & $24,3 \%$ & $6,4 \%$ \\
\hline Auf dem Arbeitsmarkt gefährdete Gruppen & $52,8 \%$ & $42,1 \%$ & $72,0 \%$ & - \\
\hline$<6$ Monate arbeitslos & $43,8 \%$ & $12,5 \%$ & $39,3 \%$ & - \\
\hline 6-12 Monate arbeitslos & $27,4 \%$ & $31,1 \%$ & $18,5 \%$ & - \\
\hline$>$ Ein Jahr arbeitslos & $28,8 \%$ & $56,4 \%$ & $42,3 \%$ & - \\
\hline
\end{tabular}

Hinweise: 1. Die VDAB-Daten spiegeln die Zusammensetzung der Bevölkerung der arbeitslosen Arbeitsuchenden im Beobachtungszeitraum wider. Der durchschnittliche prozentuale Anteil wurde ausgehend von Arvastat-Daten für den Zeitraum von Januar 2009 bis Juni 2014 berechnet. 2. Daten aus dem Jahr 2012 aus dem Aktionsplan Unternehmertum der flämischen Regierung (Ministerium für Wirtschaft, Wissenschaft und Innovation)

Die Daten zeigen, dass die Teilnehmergruppe sehr heterogen war und zu einem beträchtlichen Anteil $(52,8 \%)$ aus Menschen bestand, die zu mindestens einer der Gruppen gehörten, die gemäß der VDAB als auf dem Arbeitsmarkt „gefährdet“ gelten. Dazu zählen Menschen im Alter ab 50 Jahren (7,3\%), Menschen ohne Sekundarschulabschluss (33\%), Erwerbsunfähige (6,3\%), Menschen aus ethnischen Minderheitengruppen (18,7\%) sowie Frauen (39,4\%). Außerdem waren 28,8\% der Teilnehmer zu Beginn der ersten Programmphase mindestens ein Jahr lang arbeitslos. Vergleicht man diese Anteile mit der Bevölkerung der arbeitslosen Arbeitsuchenden, wird deutlich, dass Frauen, Menschen im Alter von 50 Jahren und älter, Menschen mit niedrigem Bildungsstand, Erwerbsunfähige, ethnische Minderheiten und Langzeitarbeitslose unterrepräsentiert waren. Verglichen mit der Bevölkerung der selbstständig Erwerbstätigen waren bei dem 
Programm Ältere unterrepräsentiert, Frauen, Menschen mit Behinderungen und ethnische Minderheiten jedoch leicht überrepräsentiert.

Tabelle 5.1 zeigt zudem einige Unterschiede in den Profilen der Teilnehmer der ersten und der zweiten Programmphase. Dank der genauen Überwachung der Entwicklung jedes Teilnehmers durch UNIZO konnte das Ausstiegsverhalten während des Programms analysiert werden. Nach der ersten Phase erhielten 386 Teilnehmer (15\%) eine negative Bewertung, 102 Teilnehmer (4\%) wurden an ein anderes Beratungsprojekt verwiesen, und 1117 Teilnehmer (45\%) entschlossen sich, aus dem Programm auszusteigen. Außerdem entschieden sich 371 (41\%) der übrigen 905 Teilnehmer, die die erste Phase abgeschlossen und eine positive Bewertung erhalten hatten, die zweite Programmphase nicht zu beginnen. Einelogistische Regressionsanalyse ergab, dass Erwerbsunfähige und ethnische Minderheiten mit signifikant höherer Wahrscheinlichkeit aus dem Programm ausstiegen (aufgrund einer negativen Bewertung oder der Versetzung in ein anderes Programm) (IDEA, 2014). Auch die Wahrscheinlichkeit, das Programm aus eigener Initiative abzubrechen, lag bei diesen Gruppen höher. Dies war auch der Fall bei den jungen Menschen unter 25 Jahren und Menschen mit niedrigem Bildungsstand (d. h. Teilnehmer ohne Sekundarschulabschluss).

\section{Projektfinanzierung}

Das Projekt „Unternehmertum funktioniert“ wurde durch drei aufeinanderfolgende Projekte des Europäischen Sozialfonds (ESF) entwickelt, die durch die ESF-Agentur Flandern, die flämische Regierung und zusätzliche Kofinanzierungen finanziert wurden. Tabelle 5.2 zeigt die Entwicklung des Haushalts über den gesamten Projektzeitraum, nämlich „Unternehmertum funktioniert I“ (1. November 2008 bis 30. September 2009), „Unternehmertum funktioniert II“ (1. Oktober 2009 bis 31. Oktober 2010) und „Unternehmertum funktioniert III“ (1. November 2010 bis 28. Februar 2013).

Tabelle 5.2. Finanzierung von „Unternehmertum funktioniert I, II und III“

\begin{tabular}{|c|c|c|c|c|}
\hline & & $\begin{array}{l}\text { Unternehmertum } \\
\text { funktioniert I (1 Jahr) }\end{array}$ & $\begin{array}{c}\text { Unternehmertum } \\
\text { funktioniert II (1 Jahr) }\end{array}$ & $\begin{array}{c}\text { Unternehmertum } \\
\text { funktioniert III (2,5 Jahre) }\end{array}$ \\
\hline \multirow{4}{*}{$\begin{array}{l}\text { Beantragter } \\
\text { Haushalt }\end{array}$} & ESF-Finanzierung & 270000 EUR & 292500 EUR & 585000 EUR \\
\hline & Flämische Kofinanzierung & 330000 EUR & 357500 EUR & 765000 EUR \\
\hline & Zusätzliche Mittel & 66692 EUR & 65000 EUR & 130000 EUR \\
\hline & Beantragter Gesamtbetrag & 666692 EUR & 715000 EUR & 1480000 EUR \\
\hline \multicolumn{2}{|c|}{ Gewährter Gesamtbetrag } & 648525 EUR & 710600 EUR & k. A. \\
\hline
\end{tabular}

Quelle: IDEA Consult basierend auf Daten der ESF-Agentur Flandern

Auf der Grundlage dieser Daten lassen sich die durchschnittlichen Kosten pro begonnenen Beratungsverlauf berechnen. Beim Projektaufruf für „Unternehmertum funktioniert (I, II und III)“ wurden durchschnittliche Kosten von 1100 EUR pro begonnene Teilnahme einkalkuliert. Diese Schätzung erweist sich als ziemlich zutreffend, wenn man den gewährten Gesamtbetrag (2839125 EUR) durch die Gesamtanzahl der Aufnahmen (d. h. 2611 Teilnehmer) dividiert (siehe Tabelle 5.3).

Tabelle 5.3. Durchschnittliche Kosten pro Beratungsverlauf in „Unternehmertum funktioniert“

\begin{tabular}{llll}
\hline & \multicolumn{1}{c}{$\begin{array}{c}\text { Unternehmertum } \\
\text { funktioniert I } \\
\text { (1 Jahr) }\end{array}$} & $\begin{array}{c}\text { Unternehmertum } \\
\text { funktioniert II } \\
\text { (1 Jahr) }\end{array}$ & $\begin{array}{c}\text { Unternehmertum } \\
\text { funktioniert III } \\
\text { (2,5 Jahre) }\end{array}$ \\
\hline Gewährter Gesamtbetrag & 648525 EUR & 710600 EUR & 1480000 EUR(') \\
$\begin{array}{l}\text { Anzahl der Teilnehmer, die die erste Programmphase begannen } \\
\begin{array}{l}\text { Durchschnittliche Kosten pro tatsächlich begonnenes Programm von } \\
\text { zwölf Monaten (Phase 1) }\end{array}\end{array}$ & 442 & 475 & 1184 \\
\hline
\end{tabular}

Hinweise: 1. Zum Zeitpunkt der Evaluierung lag die Abschlussrechnung für „Unternehmertum funktioniert III“ noch nicht vor. Aus diesem Grund wurde für diese Berechnung der geplante Haushalt verwendet.

Quelle: IDEA Consult basierend auf Daten der ESF-Agentur Flandern 


\section{Herausforderungen}

Da das Projekt „Unternehmertum funktioniert“ drei Generationen von ESF-Projekten umfasste, konnte bei der Entwicklung der Beratungskomponente eine Reihe von Lerneffekten berücksichtigt werden.

Erstens war es für „Unternehmertum funktioniert“ schwierig, einige der Unternehmerkandidaten zu erreichen, zum Beispiel Erwerbsunfähige und ethnische Minderheitengruppen. Die Durchführung aller Schulungskurse auf Niederländisch stellte viele Unternehmerkandidaten vor eine erhebliche sprachliche Hürde, auch wenn im Hauptkurs zur Betriebswirtschaft ein Sprachtrainer für Nichtmuttersprachler tätig war. Außerdem war es im gesamten Verlauf der drei Programmdurchführungen durchgehend schwierig, die Gruppe der Unternehmer zu erreichen, die Konkurs gemacht hatten.

Eine zweite Herausforderung bestand darin, ein professionelles Coaching für 650 Teilnehmer bereitzustellen. Ein Team bestehend aus nur fünf Betreuern war für die Durchführung der gesamten Beratungskomponente zuständig. Obwohl der Weg bis zur Gründung des neuen Unternehmens klar vorgezeichnet war, wurde jeder Unternehmerkandidat sowohl einzeln als auch in der Gruppe zusammen mit anderen Unternehmerkandidaten betreut. Da jeder Gründungsprozess sehr spezifisch ist, waren die Umsetzung des Programms und der Schulungsbedarf der Teilnehmer je nach Situation der Unternehmerkandidaten sehr unterschiedlich. Der flexible Ansatz stellte eine Herausforderung für das kleine Betreuerteam dar, insbesondere weil jeder Teilnehmer auf einfache und schnelle Weise mit seinem persönlichen Betreuer Kontakt aufnehmen können sollte.

Eine dritte Herausforderung bestand darin, eine angemessene Balance zwischen der Notwendigkeit der Förderung von Unabhängigkeit und Kreativität einerseits und der Vorgabe einer ausreichenden Struktur andererseits zu finden, um die Teilnehmer durch das Programm zu leiten. In einigen Fällen nährten die persönlichen Beziehungen eine Abhängigkeit, die die Fähigkeit des betroffenen Teilnehmers beeinträchtigte, unternehmerische Fähigkeiten zu entwickeln und andere Ressourcen zu nutzen.

\section{Auswirkungen}

Da es sich bei „Unternehmertum funktioniert“ um ein Aktivierungsprojekt handelte, wurden die Auswirkungen in erster Linie durch die Evaluierung des Übergangs der Teilnehmer in eine selbstständige Erwerbstätigkeit (und abhängige Erwerbstätigkeit) bewertet. Tabelle 5.4 zeigt die Übergangsquoten der Teilnehmer, die das Programm abschlossen, und der Teilnehmer, die das Programm abbrachen. Für die meisten Teilnehmer fällt die Messung nach sechs Monaten mit dem Ende des Programms zusammen (da die zweite Phase bis zu sechs Monate dauern konnte). Somit gibt die Messung nach 18 Monaten die Ergebnisse ein Jahr nach Abschluss des Programms an.

Das Programm hatte insofern große Auswirkungen, als dass es vielen Leuten den Weg zurück in eine Beschäftigung ermöglichte. Die durchschnittliche Quote des Übergangs in eine Beschäftigung (gemessen ein Jahr nach der Meldung bei derVDAB) lag bei den arbeitslosen Arbeitsuchenden (in den flämischen Provinzen insgesamt) im Zeitraum 2011-2013 bei 52,4\%. Das Programm erzielte Übergangsquoten von 66,6\% nach einem Jahr bei denjenigen, die eine selbstständige Erwerbstätigkeit anstrebten, und 81,3\% bei denjenigen, die nach dem Abschluss mindestens der ersten Programmphase eine Beschäftigung anstrebten.

Eine logistische Regressionsanalyse ermittelte die Faktoren, die zur Wahrscheinlichkeit, eine abhängige oder selbstständige Erwerbstätigkeit zu finden, beitrugen (IDEA, 2014). Die Analysen wurden für vier Teilnehmergruppen durchgeführt:

1. Alle Teilnehmer, die die erste Programmphase begannen;

2. Unternehmerkandidaten, die das Aufnahmegespräch nicht bestanden oder an ein anderes Beratungsprojekt verwiesen wurden;

3. Teilnehmer, die das Programm vollständig abschlossen; und

4. Teilnehmer, die das Programm nicht abschlossen. 
Tabelle 5.4. Anzahl der Teilnehmer an „Unternehmertum funktioniert“, die in eine selbstständige oder abhängige Erwerbstätigkeit übergingen

\begin{tabular}{|c|c|c|c|}
\hline & & $\begin{array}{l}\text { Monate nach } \\
\text { Abschluss der } \\
\text { ersten Phase }\end{array}$ & $\%$ \\
\hline \multirow{6}{*}{$\begin{array}{l}\text { Alle Teilnehmer, die mindestens die erste } \\
\text { Programmphase abschlossen }\end{array}$} & \multirow[t]{3}{*}{ Übergang in eine abhängige Erwerbstätigkeit } & 6 Monate & 73,6 \\
\hline & & 12 Monate & 81,3 \\
\hline & & 18 Monate & 83,7 \\
\hline & \multirow[t]{3}{*}{ Übergang in eine selbstständige Erwerbstätigkeit } & 6 Monate & 61,4 \\
\hline & & 12 Monate & 66,6 \\
\hline & & 18 Monate & 63,8 \\
\hline \multirow{6}{*}{$\begin{array}{l}\text { Gefährdete Gruppen, die mindestens die erste } \\
\text { Programmphase abschlossen }\end{array}$} & \multirow[t]{3}{*}{ Übergang in eine abhängige Erwerbstätigkeit } & 6 Monate & 74,1 \\
\hline & & 12 Monate & 79,4 \\
\hline & & 18 Monate & 82,4 \\
\hline & \multirow[t]{3}{*}{ Übergang in eine selbstständige Erwerbstätigkeit } & 6 Monate & 62,2 \\
\hline & & 12 Monate & 64,2 \\
\hline & & 18 Monate & 62,4 \\
\hline \multirow[t]{6}{*}{ Teilnehmer, die das Programm abbrachen } & \multirow[t]{3}{*}{ Übergang in eine abhängige Erwerbstätigkeit } & 6 Monate & 46,5 \\
\hline & & 12 Monate & 55,8 \\
\hline & & 18 Monate & 57,8 \\
\hline & \multirow[t]{3}{*}{ Übergang in eine selbstständige Erwerbstätigkeit } & 6 Monate & 10,7 \\
\hline & & 12 Monate & 13,8 \\
\hline & & 18 Monate & 15,0 \\
\hline
\end{tabular}

Quelle: Überwachungsdaten der PES (VDAB), verarbeitet durch IDEA Consult

Die Ergebnisse der Analysen unter allen Teilnehmern, die die erste Phase von „Unternehmertum funktioniert" begannen, zeigen deutlich, dass ein Abschluss des Programms einen starken, positiven Zusammenhang mit der Wahrscheinlichkeit hatte, eine abhängige Erwerbstätigkeit zu finden, sowie mit den Aussichten, eine selbstständige Erwerbstätigkeit aufzunehmen. Allerdings konnte durch die Analyse nicht ermittelt werden, ob dies dem Inhalt des Programms zuzuschreiben oder einem Selektionsfehler geschuldet war, denn mit diesen Daten ließ sich nicht feststellen, ob nur die stärkeren Profile Zugang zum Programm erhielten.

Die soziodemografischen Merkmale, die die Wahrscheinlichkeit eines Übergangs in eine abhängige oder selbstständige Erwerbstätigkeit bestimmen, unterschieden sich je nach Umfang der Erfahrungen mit dem Programm:

- Bei den Teilnehmern, die das Programm abschlossen, schienen die weiblichen Unternehmerkandidaten und Teilnehmer, die zu Programmbeginn mindestens ein Jahr lang arbeitslos waren, sechs bzw. zwölf Monate nach Abschluss des Programms signifikant weniger häufig den Übergang in die abhängige oder selbstständige Erwerbstätigkeit geschafft zu haben.

- Bei Unternehmerkandidaten, die ihre Teilnahme an dem Programm beendeten, stellte sich heraus, dass sie mit geringerer Wahrscheinlichkeit eine Beschäftigung fanden, wenn sie erwerbsunfähig, älter als 50 Jahre oder mindestens ein Jahr lang arbeitslos waren. Zusätzlich zu den Erwerbsunfähigen nahmen Menschen mit einem niedrigen Bildungsstand weniger häufig eine selbstständige Erwerbstätigkeit auf, wenn sie aus dem Programm ausstiegen.

- Unter den Teilnehmern, die in ein anderes Beratungsprojekt wechselten, lag die Wahrscheinlichkeit, den Übergang in eine abhängige Erwerbstätigkeit zu schaffen, bei Erwerbsunfähigen, ethnischen Minderheiten, Menschen über 50 Jahre und Arbeitslosen signifikant niedriger. Allerdings war in dieser Teilnehmergruppe nur bei den Menschen ohne Sekundarschulabschluss die Wahrscheinlichkeit der Aufnahme einer selbstständigen Erwerbstätigkeit signifikant geringer. 


\section{Textfeld 5.1. Blick in die Zukunft: Unternehmertum funktioniert - die Fortsetzung}

Nach drei Generationen von „Unternehmertum funktioniert“ wurde im Oktober 2013 ein neues Coaching-Projekt ins Leben gerufen, um arbeitslosen Arbeitsuchenden bei der Gründung eines eigenen Unternehmens zu helfen: „Bringen Sie Ihr Unternehmen zum Laufen“ („Maak werk van je zaak“). Wie „Unternehmertum funktioniert" wird auch dieses Programm durch die ESF-Agentur Flandern mit Syntra-Limburg als Träger finanziert. Wie „Unternehmertum funktioniert“ richtet es sich an arbeitslose Arbeitsuchende, aber auch an Menschen, die von Massenentlassungen bedroht sind.

Die wichtigsten Merkmale von „Unternehmertum funktioniert“ finden sich auch in diesem neuen Programm wieder: Es besteht aus zwei Phasen und dauert maximal ein Jahr. Vor dem Einstieg in das Programm werden in den regionalen Geschäftsstellen von Syntra erste Screenings und Informationssitzungen durchgeführt. Diese werden wöchentlich ausgerichtet. Bestehen Unternehmerkandidaten die Aufnahme, gelangen sie in die Orientierungsphase (bis zu sechs Monate), in der es um die Erstellung eines persönlichen Schulungs- und Aktionsplans geht. Diese Phase resultiert in einem Geschäftsplan, einer Machbarkeitsstudie, und - sofern keine Ausnahme gemacht wird - einem betriebswirtschaftlichen Zertifikat. Die zweite Phase ist die Coaching-Phase (bis zu sechs Monate). In dieser Phase geht es um die Umsetzung des persönlichen Aktionsplans mit konkreten Maßnahmen in Richtung der eigentlichen Unternehmensgründung.

Ein wichtiger Unterschied zwischen diesem Programm und „Unternehmertum funktioniert“ ist, dass jeder Teilnehmer während des ganzen Programms Anspruch auf ein maßgeschneidertes Coaching hat. Dies wird durch einen „virtuellen Rucksack“ (d. h. Finanzmittel für Schulungen) in Höhe von 500 EUR für Schulungen aus einem umfassenden Menü ermöglicht. Dieses Menü umfasst kurzfristige Schulungsinitiativen von privaten und öffentlichen Anbietern.

Die Gesamtmittel für dieses Programm betragen 600000 EUR für das Coaching von 800 Unternehmerkandidaten und 400000 EUR für die Schulungsrucksäcke (d. h. 500 EUR pro Teilnehmer). Da das Programm viele Unternehmerkandidaten für sich gewinnen konnte, wurden sowohl die Ziele als auch die Finanzierung erhöht. Seitdem die anfänglichen 800 Teilnehmer im Juni 2014 starteten, wurden weitere 334147 EUR für das Coaching von 250 zusätzlichen Teilnehmern zuzüglich 125000 EUR zur Finanzierung der Schulungsrucksäcke einkalkuliert. Diese Haushaltserhöhung wurde durch die VDAB finanziert. Wird der Haushalt für die Schulungsrucksäcke nicht voll ausgeschöpft, wird er zur Finanzierung eines Zusatzprogramms eingesetzt.

Die Aufnahmefrist für „Bringen Sie Ihr Unternehmen zum Laufen“ endete im Juni 2015. Mehr als 1600 arbeitslose Arbeitsuchende konnten erreicht werden (doppelt so viele wie ursprünglich geplant). Davon gehörten $43 \%$ Gruppen an, die auf dem Arbeitsmarkt gefährdet sind. Dank dem Coaching und der Betreuung nahmen rund 400 arbeitslose Arbeitsuchende eine selbstständige Erwerbstätigkeit auf. Das entspricht einer Erfolgsquote von $25 \%$.

Ausgehend von diesem Erfolg wurde ein neues Projekt mit dem Titel „Bringen Sie Ihr Unternehmen zum Laufen 2“ ins Leben gerufen, das vom ESF finanziert und durch die VDAB, SYNTRA Flandern und Enterprise Flandern kofinanziert wird. Von Juli 2015 bis Ende 2017 betreut der Träger SYNTRA Limburg 1250 bis 1500 arbeitslose Arbeitsuchende bei der Gründung eines Unternehmens.

\section{Voraussetzungen für die Übertragung}

Wesentliche Merkmale der Gestaltung von „Unternehmertum funktioniert“, die für die Übertragung dieses Ansatzes auf einen anderen Kontext von Bedeutung sein könnten, sind folgende:

1. Ein gut entwickeltes Netzwerk aus Partnern. Es bestand eine starke Partnerschaft zwischen mehreren Organisationen (d. h. UNIZO, VDAB und Syntra). Die enge Zusammenarbeit mit der VDAB erleichterte den Zugang zur Zielgruppe (Arbeitsuchende), während die Partnerschaft mit Syntra Möglichkeiten zur Stärkung von Kompetenzen eröffnete. Da sich Syntra in erster Linie auf unternehmerische Schulungen konzentriert, ermöglichten die gemeinsam durchgeführten Aktivitäten den Teilnehmern von „Unternehmertum funktioniert“ die Kontaktaufnahmen zu anderen Unternehmerkandidaten. Auf diese Weise stimulierte die Partnerschaft die Netzwerkarbeit und die gegenseitige Unterstützung unter Gleichgestellten. Außerdem schaffte sie direkte 
Verbindungen zu Experten. Durch die Nutzung eines solchen Expertennetzwerks konnten die Betreuer von „Unternehmertum funktioniert“ die Beratungsverläufe individuell auf die Bedürfnisse jedes einzelnen Unternehmerkandidaten zuschneiden.

2. Fortwährender Zugang zu Leistungen. Ein wichtiges Merkmal des Programms bestand darin, dass die Teilnehmer weiterhin Anspruch auf Arbeitslosenunterstützung hatten. Das finanzielle Risiko, das mit der Gründung eines Unternehmens verbunden ist, stellt eine erhebliche Hürde beim Schritt in Richtung Unternehmertum dar. Auch wenn sich die Arbeitslosenunterstützung mit der Zeit verringert, ist diese Art von finanzieller Unterstützung wesentlich, um Menschen zu ermutigen, dem Programm eine Chance zu geben, und sie im Laufe der Entwicklung ihres Unternehmens zu unterstützen.

3. Hochwertige Betreuer. Während der gesamten Evaluierung wurde die wichtige Rolle der Betreuer in dem Programm immer wieder betont. Das Wissen der Betreuer über das Unternehmertum war mindestens genauso wichtig wie ihre Erfahrungen als Berater. Dies deutet darauf hin, dass Betreuer über potenzielle Partner auf dem Markt Bescheid wissen müssen und innerhalb von Unterstützungsorganisationen über Kontakte verfügen müssen, um die Kandidaten während oder sogar nach dem Programm wirksam weiterzuvermitteln. Idealerweise werden die Teilnehmer mit den richtigen Menschen innerhalb der Organisationen zusammengebracht, die effiziente, weitergehende Hilfestellung leisten können.

\section{Literatur}

Access to finance - Meeting Flanders - 12. und 13. Mai 2011, verfügbar unter: www.cop-ie.eu/sites/ default/files/TG_Access_to_Finance-Meeting_Report_Flanders_12th_and_13th_may.pdf.

Bundesministerium für Arbeit und Soziales (2010), „Aus der Arbeitslosigkeit in die Selbstständigkeit im Aufschwung Gründungen fördern“. Internationale Konferenz, veranstaltet vom Bundesministerium für Arbeit und Soziales (BMAS) in Zusammenarbeit mit der OECD (ELSA und LEED), Berlin, 7. bis 8. Oktober 2010, verfügbar unter: http://www.bmas.de/SharedDocs/ Downloads/DE/PDF-Publikationen/a809-umbruch-aus-der-arbeitslosigkeit-in-die-selbststaendigkeit. pdf?_blob=publicationFile\&v=2.

IDEA Consult (2014), „Maatpakbegeleiding werkzoekenden naar ondernemerschap“ (Auf die Bedürfnisse der Arbeitslosen zugeschnittene unternehmerische Beratung), Arbeitsmarkt-Forschungsprogramm Viona der flämischen Regierung.

UNIZO (kein Datum), „UNIZO as an entrepreneurial organization“, verfügbar unter: www.letsmovethemarket.eu/website/images/pdf/Presentation\%20Ilse\%20Claes_7nou.pdf. 


\section{Frauen auf dem Arbeitsmarkt, Kroatien}

In dieser Fallstudie wird ein Beispiel für einen integrierten Ansatz vorgestellt, um arbeitslose Frauen bei der Unternehmensgründung als Weg zurück in den Arbeitsmarkt zu unterstützen. Die Beschreibung behandelt die Ziele und den Hintergrund des Projekts und skizziert die durchgeführten Hauptaktivitäten. Zudem werden die größten Herausforderungen und die Auswirkungen des Projekts vorgestellt. Auch die Voraussetzungen für die Übertragung dieser Erfahrung auf einen anderen Kontext werden erörtert.

\section{Ziele}

Das Projekt „Frauen auf dem Arbeitsmarkt“ wurde von der kroatischen Arbeitsverwaltung (HZZ, Hrvatski zavod za zapošljavanje) im Rahmen des Instruments für Heranführungshilfe (IPA), 4. Komponente „Förderung der Humanressourcen“ im Zeitraum 2007-2009 durchgeführt. Die Projektumsetzung begann im September 2010 und endete im März 2012. Dazu gehörte ein Beihilfeprogramm zur Unterstützung arbeitsloser Frauen bei der Unternehmensgründung als ein Weg zurück in den Arbeitsmarkt sowie ein technisches Unterstützungsprogramm für politische Entscheidungsträger.

Das übergeordnete Ziel des Projekts war die Steigerung der Beschäftigungsfähigkeit benachteiligter Frauen und die Förderung ihres Einstiegs in den Arbeitsmarkt. Die Zielgruppe dieser Beihilfe waren arbeitslose Frauen über 40 Jahre, Frauen, die seit mindestens zwölf Monaten arbeitslos waren, nicht erwerbstätige Frauen, arbeitslose Frauen aus Gespanschaften mit überdurchschnittlicher Arbeitslosenquote sowie arbeitslose Frauen aus ethnischen Minderheitengruppen, wobei der Schwerpunkt auf der Roma-Minderheit lag.

\section{Hintergrund}

Der Hauptgrund für die Einführung dieser Initiative war dieVerringerung der Benachteiligungen auf dem Arbeitsmarkt, denen arbeitslose Kroatinnen gegenüberstehen. Wichtige Arbeitsmarktindikatoren zeigen, dass die Ungleichheit zwischen den Geschlechtern zwar weniger geworden ist, jedoch nach wie vor geschlechtsspezifische Unterschiede vorhanden sind. So lag zum Beispiel im Jahr 2014 die Beschäftigungsquote der Frauen 9,1 Prozentpunkte unter der der Männer. Zudem liegen in Kroatien die Erwerbstätigkeitsquoten und Monatseinkommen bei Frauen niedriger als bei den Männern. Eine Vielzahl von Faktoren sorgt dafür, dass die Frauen größere Schwierigkeiten beim Zugang, bei der Rückkehr und beim Verbleib im Arbeitsmarkt haben. Ein überproportionaler Anteil der Frauen ist in befristeten und unsicheren Arbeitsplätzen tätig, was dazu führt, dass sie gefährdeter und stärker von Armut oder externen Schocks, zum Beispiel der aktuellen Wirtschaftskrise, betroffen sind.

Benachteiligte Frauen erleben diese Probleme noch intensiver. Arbeitslose Frauen, insbesondere diejenigen zwischen 40 und 65 Jahren, stehen vor Hindernissen in Bezug auf die Suche und die Sicherung 
einer Beschäftigung, nicht nur aufgrund der allgemein ungünstigen Konjunktur, sondern auch, weil ihnen Bildung und Fähigkeiten fehlen. In Ermangelung an Systemen zum lebenslangen Lernen verringert sich die Wahrscheinlichkeit, dass sich diese Lücke nach und nach schließen kann.

Ziel des Programmswareine Reaktion aufdiesemUmstand,indemeineReihegleichstellungsorientierter und maßgeschneiderter Arbeitsmarktpolitiken für benachteiligte Frauen finanziert wurden, darunter Frauen über 40 Jahre, Langzeitarbeitslose, Frauen, die in den Gespanschaften mit den höchsten Arbeitslosenquoten leben, und Frauen, die aus Minderheitsbevölkerungen wie die der Roma stammen.

Das Programm insgesamt band lokale Partner der HZZ bei der Gestaltung und Umsetzung der Unterstützung für Frauen auf dem Arbeitsmarkt ein, unter anderem die regionalen Geschäftsstellen der HZZ, Sozialhilfezentren, Stadtverwaltungen, die zuständigen staatlichen Agenturen, Gewerkschaften, Arbeitgeberverbände und Vertreter der Zivilgesellschaft.

\section{Aktivitäten}

Das Beihilfeprogramm wurde im August 2009 mit einem Aufruf zur Einreichung von Vorschlägen ins Leben gerufen, der auf der EuropeAid- und HZZ-Website veröffentlicht wurde. Infrage kommende Bewerberorganisationen konnten sich einzeln oder in Partnerschaften für zwölfmonatige Projekte mit einer Finanzierung von 90000 bis 140000 EUR für die folgenden Arten von Aktivitäten bewerben (die Liste war nicht erschöpfend, und geeignete Maßnahmen, die nicht aufgeführt wurden, konnten ebenfalls für eine Unterstützung in Betracht kommen):

- Bereitstellung von Hilfe und Informationen zu Möglichkeiten auf dem Arbeitsmarkt sowie Anreizmaßnahmen für Beschäftigung;

- Aktivitäten, die auf die Entwicklung neuer Methoden für Verfahren und Dienste gerichtet sind, die benachteiligten Gruppen von Frauen den Zugang zum Arbeitsmarkt erleichtern sollen;

- Aktivitäten, die auf die Erhöhung der Beschäftigungsfähigkeit von benachteiligten Gruppen von Frauen gerichtet sind;

- Aktivitäten, die auf die Schaffung von Netzwerken, Partnerschaften und neuen Methoden zur Unterstützung von benachteiligten Gruppen von Frauen gerichtet sind.

55 Projektbewerbungen gingen ein und wurden einer dreistufigen Beurteilung unterzogen: i) Einhaltung von Verwaltungsvorschriften (z. B. vollständige Bewerbung, rechtzeitiger Eingang), ii) Evaluierung des Konzepts (z. B. Relevanz, Vorzüge, Wirksamkeit, Tragfähigkeit und Nachhaltigkeit der vorgeschlagenen Maßnahme) und iii) Evaluierung der vollständigen Bewerbung (z. B. Überprüfung der Qualität anhand von vorab definierten Evaluierungskriterien, einschließlich Haushalt, Kapazität der Bewerber und ihrer Partner). Der Evaluierungsprozess dauerte neun Monate und wurde mit Unterstützung externer Beurteilender, unter anderem Vertreter der für den Schwerpunkt des Beihilfeprogramms zuständigen Fachministerien, von der ausschreibenden Stelle durchgeführt.

In diesem Prozess wurden 18 Vorschläge ausgewählt, denen im November 2010 eine Finanzierung gewährt wurde. Drei dieser Projekte waren so angelegt, dass sie die Arbeitslosigkeit benachteiligter Frauen durch die Bereitstellung von Unterstützung für eine selbstständige Erwerbstätigkeit angingen, und zwar:

- das Projekt „Sei unternehmerisch - sei wettbewerbsfähig“ in der Gespanschaft Brod-Posavina;

- das Projekt „Schritt für Schritt in den Arbeitsmarkt“ in der Gespanschaft Vukovar-Srijem;

- das Projekt „MOTS - Mehr Möglichkeiten durch neue Fähigkeiten“ in der Gespanschaft DubrovnikNeretva.

Die Hauptinstrumente, die diese Projekte zur Förderung des weiblichen Unternehmertums einsetzten, waren folgende:

- Bereitstellung von Informationen und Schulungen zu grundlegenden unternehmerischen Fähigkeiten, Geschäftsplanung, Betriebswirtschaft, Marketing, Buchhaltung, Finanzplanung, Computerkenntnisse und Kommunikation;

- Entwicklung von Veröffentlichungen, Handbüchern und Websites;

- Ausrichtung von Treffen zwischen Unternehmen, um Projektteilnehmer und potenzielle Arbeitgeber zusammenzubringen. 
Das Projekt „Sei unternehmerisch - sei wettbewerbsfähig“ (91605 EUR) wurde von der Gespanschaft Brod-Posavina in Partnerschaft mit der regionalen HZZ-Geschäftsstelle in Slavonski Brod durchgeführt. Es beabsichtigte, 48 arbeitslose Frauen bei der aktiven Stellensuche und derVerfolgungeiner unternehmerischen Tätigkeit zu unterstützen. Zur Teilnahme an dem Projekt eingeladen wurden arbeitslose Frauen, die in der HZZ-Datenbank der Gespanschaft Brod-Posavina gemeldet waren (415 Frauen). Auf der Grundlage einer Befragung der Teilnehmerinnen wählte das Projekt eine Gruppe von Frauen (55 von 131, die Interesse bekundeten) aus, an dem Bildungsprogramm zur Stärkung der Kompetenzen für den Arbeitsmarkt und der IKT-Fertigkeiten (alle 55 Teilnehmerinnen) sowie zur Unternehmensgründung (21 Teilnehmerinnen mit Geschäftsideen) teilzunehmen. Das Projekt entwickelte außerdem öffentlichkeitswirksame Maßnahmen (z. B. Flyer, Poster und eine Website) sowie die Broschüre „Praktischer Wegweiser zum Unternehmertum für Frauen“. Laut Überwachungsdaten gründeten 13 der Teilnehmerinnen ein eigenes Unternehmen oder fanden eine Beschäftigung.

Das Projekt „Schritt für Schritt in den Arbeitsmarkt“ (110268 EUR) wurde von der regionalen HZZGeschäftsstelle in Vukovar in Zusammenarbeit mit zwei lokalen zivilgesellschaftlichen Organisationen, dem Frauenverband und dem Zentrum für soziale Bildung PRONI, durchgeführt. Der Schwerpunkt des Projekts lag auf langzeitarbeitslosen Frauen in der Gespanschaft Vukovar-Srijem. Sie sollten beim Erwerb neuer Fähigkeiten und Kompetenzen, die ihnen mehr Beschäftigungsmöglichkeiten verschaffen würden, und bei der Entwicklung innovativer Geschäftsmodelle für eine selbstständige Erwerbstätigkeit unterstützt werden. Als vorbereitende Aktivität absolvierten die Betreuer in der regionalen HZZ-Geschäftsstelle eine Schulung. Darauf folgte eine Reihe von Workshops mit den Teilnehmerinnen zu den Themen Motivation für die aktive Stellensuche, IKT-Fertigkeiten und Unternehmensgründung sowie psychologische Betreuung. Insgesamt nahmen 262 Frauen an dem Projekt teil. Davon nahmen 88 an den MotivationsWorkshops, 155 an der psychologischen Betreuung, 57 an den IKT-Workshops und 28 Frauen an den Workshops zum Unternehmertum teil. Das Projekt führte zur Gründung von zwei Unternehmen (in denen 13 Teilnehmerinnen eine Beschäftigung fanden) und der Gründung einer zivilrechtlichen Organisation durch drei Teilnehmerinnen. Zusätzlich fanden 27 Frauen eine anderweitige Beschäftigung.

Das Projekt „MOTS - Mehr Möglichkeiten durch neue Fähigkeiten“ (107539 EUR) wurde von der zivilrechtlichen Organisation Deša in Zusammenarbeit mit der regionalen HZZ-Geschäftsstelle in Dubrovnik und dem Unternehmerzentrum Dubrovnik durchgeführt. Der Zweck des Projekts war die Erhöhung der Beschäftigungsfähigkeit von 120 langzeitarbeitslosen Frauen über 40 Jahren in der Gespanschaft DubrovnikNeretva. Die Aktivitäten umfassten Seminare zum Unternehmertum und zu Finanzen und Buchhaltung sowie Schulungen zu IKT und zur Entwicklung und Bewerbung authentischer lokaler Souvenirs und Produkte. Außerdem richtete das Projekt ein B2B-Treffen aus, an dem die arbeitslosen Teilnehmerinnen lokalen Arbeitgebern ihre Fähigkeiten präsentieren und ihre Lebensläufe vorlegen konnten. 233 arbeitslose Frauen nahmen an dem Projekt teil. Von diesen fanden 59 eine Beschäftigung und neun Frauen gründeten ein Unternehmen.

Der Gesamtwert der Mittel, die für die im Rahmen des Beihilfeprogramms durchgeführten Projekte ausgeschrieben waren, belief sich auf 2017480 EUR. Das Programm konnte nur für 70\% bis 85\% der förderfähigen Gesamtkosten eine finanzielle Förderung bereitstellen, sodass die Bewerber den Restbetrag aus eigenen Mitteln oder anderen Quellen von außerhalb der Europäischen Union kofinanzieren mussten. In der Praxis finanzierte das Beihilfeprogramm zwischen 77\% und 85\% jedes Projekts, dem eine Finanzierung gewährt worden war.

\section{Herausforderungen}

Alle Beihilfeempfänger durchliefen eine Reihe vorbereitender Workshops und Konsultationen, und die ausschreibende Stelle stand den Beihilfeempfängern bei Klärungsbedarf und zur Unterstützung zur Verfügung. Dennoch stießen die Träger des Projekts auf Schwierigkeiten. Von dem ausgeschriebenen Gesamtbetrag von 2017480 EUR für alle 18 Beihilfeverträge wurden nur 1853606 EUR (91,8\% des ausgeschriebenen Gesamtbetrags) auch tatsächlich ausbezahlt. Der Hauptgrund für diese Auszahlungslücke bestand in der unzureichenden Planung und Finanzverwaltung der Beihilfehaushalte seitens der Bewerber. Dies zog eine Verringerung der förderfähigen Gesamtkosten nach sich. 


\section{Auswirkungen}

Dieses Programm ermöglichte zahlreichen Einrichtungen auf nationaler, regionaler (d. $\mathrm{h}$. gespanschaftlicher) und lokaler Ebene die Entwicklung neuen Wissens und die Unterstützung von Maßnahmen für verschiedene gefährdete Gruppen, die für den Arbeitsmarkt von Bedeutung sind, insbesondere Frauen. Ein weiteres Schlüsselergebnis war, dass der Aufruf zur Einreichung von Vorschlägen für das Programm ausreichend spezifisch war, um Gebote aus allen Teilen des Landes zu stimulieren, aber auch flexibel genug, um den bietenden Einrichtungen die Bildung von Bietergemeinschaften und die Entwicklung von Geboten zu erlauben, die ihrer Ansicht nach den Bedürfnissen ihrer Gemeinschaften und Interessenträger am besten entsprachen. Dies spielte eine wichtige Rolle bei der Entwicklung und Umsetzung innovativer Vorschläge im Rahmen der vorgegebenen Kriterien. So konnte die HZZ aus den gewonnenen Erfahrungen lernen.

In Bezug auf die Klienten halfen die drei Projekte zur Unterstützung arbeitsloser Frauen bei der Unternehmensgründung 105 Frauen dabei, eine selbstständige oder abhängige Erwerbstätigkeit aufzunehmen.

\section{Voraussetzungen für die Übertragung}

Es ist wichtig anzuerkennen, dass sich Kroatien zu dem Zeitpunkt, als diese Initiative konzipiert und umgesetzt wurde, im Beitrittsprozess zur Europäischen Union befand. Die Initiative sollte die relevanten Interessenträger dafür sensibilisieren, wie wichtig es ist, sich in verschiedenen Kategorien auf arbeitslose Frauen zu konzentrieren und geeignete politische Initiativen zu entwickeln. Trotz dieses spezifischen Kontextes bietet die Erfahrung zweierlei wichtige Erkenntnisse für die Anwendung eines ähnlichen Ansatzes an anderen Orten:

1. Einsatz von Aufrufen zur Einreichung von Vorschlägen, um den Privatsektor einzubinden. Dies räumte den Bietern eine angemessene Flexibilität ein, um die Kriterien so auszulegen, dass sie den tatsächlichen Bedürfnissen ihrer Gemeinschaften und Interessenträger optimal entsprachen, und regte damit alternative Ansätze und Innovationen an. Ein derartiger Ansatz kann besonders im Kontext gefährdeter Gruppen, die äußerst schwierig zu erreichen sind, von Bedeutung sein. Allerdings sind die Vergabeverfahren der Europäischen Union für alle Beteiligten, einschließlich der Behörden und Bieter, anspruchsvoll. Entscheidend ist, dass bei der Umsetzung von Systemen mit unerfahrenen Nutzern Schulungen und Unterstützung bereitgestellt werden.

2. Vorausplanung für Überwachung und Evaluierung. Fehlende Überlegungen und fehlende Priorisierung beim Aspekt der Überwachung und Evaluierung stellten eine der größten Schwächen dieser Erfahrung dar. Bei einer so großen Anzahl von Projekten, Trägern und Nutzern war es nicht möglich, Überwachungs- und Evaluierungssysteme hinterher konsequent umzusetzen. 


\section{Literatur}

Kroatische Arbeitsverwaltung (2010), „Grant contracts awarded during November 2010”, verfügbar unter: www.hzz.hr/dfc/userdocsimages/AN-129003(GS).pdf.

Kroatische Arbeitsverwaltung, Abteilung für Finanzierung und die Vergabe von EU-Projekten (2009), „Frauen auf dem Arbeitsmarkt-Richtlinien für Antragsteller“, verfügbar unter: www.hzz.hr/dfc/userdocsimages/// GfA-129003(GS)-CRO.pdf.

Europäische Kommission (2012), „The current situation of gender equality in Croatia - Country Profile 2012“, Generaldirektion Justiz.

Europäische Kommission (2007), „Croatia: Operational Programme for Human Resources Development 2007 - 2009“, verfügbar unter: http://ec.europa.eu/enlargement/pdf/croatia/ipa/4_croatia_human_resources_ development_operational_programme_2007-2009_en.pdf .

GEM (2012), „Što čini Hrvatsku (ne)poduzetničkom zemljom? (Was macht Kroatien zu einem (nicht) unternehmerischen Land?)“, verfügbar unter: www.gemconsortium.com.

WYG International Ltd. (2012), „Final Report - Women in the Labour Market“. 


\title{
Geschäftscoaching für ethnische Minderheiten, Dänemark
}

\begin{abstract}
In dieser Fallstudie wird ein Unternehmercoaching-Projekt für Unternehmer aus ethnischen Minderheitengruppen vorgestellt. Die Fallstudie erörtert die Ziele, den Hintergrund, die Aktivitäten sowie die Auswirkungen des Projekts. Außerdem werden die bei der Durchführung des Projekts sichtbar werdenden Herausforderungen und die Voraussetzungen für die Übertragung dieses Projekts auf einen anderen Kontext präsentiert.
\end{abstract}

\section{Ziele}

Das Ziel des Geschäftscoaching-Programms im dänischenVejle besteht darin, potenzielle Unternehmer aus ethnischen Minderheitengruppen, die in der Stadt ansässig sind, zu betreuen. Der Betreuer stellt Informationen nicht nur zur Unternehmensförderung, sondern auch im Zusammenhang mit Einwanderung, Gesundheit, Unterkunft und sonstigen Aspekten des Lebens in einem fremden Land wie Dänemark zu Verfügung. Bei Bedarf findet eine Weitervermittlung an formelle Unternehmensförderungsorganisationen und andere Dienstleister statt. Das Projekt bietet keine finanzielle Unterstützung, kann Klienten jedoch an andere Anbieter weitervermitteln und Beratung zur Vorstellung von Geschäftsplänen bei Darlehensgebern leisten.

In Vejle lebt eine wachsende ethnische Minderheitengemeinschaft, die in den 1990er-Jahren vor allem aus der Türkei stammte. Andere Zuwanderer kamen aus Teilen Nordafrikas, insbesondere Tunesien, Marokko und Algerien. In den letzten Jahren und insbesondere seit 2003 kommen die Zuwanderer aus immer mehr unterschiedlichen Ländern. Immer mehr kommen aus den neuen Mitgliedstaaten der Europäischen Union, vor allem aus den baltischen Staaten und Polen.

Das Coaching-Projekt startete 2004 und unterstützt auch heute noch Unternehmer aus ethnischen Minderheitengruppen. Es begann als informelle Initiative und baute auf der Erfahrung seines Gründers Muhsin Türkyilmaz auf, der bereits Freunde und Kontakte auf informeller Basis beriet. Da er Dänisch sprach, wurde er häufig gebeten, Menschen auf Behördengängen zu begleiten, wo er entweder als Dolmetscher auftrat oder den Behörden die Situation erläuterte. In dieser Funktion verdiente er sich das Vertrauen innerhalb seiner Gemeinschaft. Er fand nach und nach heraus, dass viele Zuwanderer die Gründung eines eigenen Unternehmens anstrebten, da es so schwierig für sie war, eine allgemeine Beschäftigung zu finden. Einige von ihnen waren in ihrem Heimatland Unternehmensinhaber gewesen und hatten schon durch ihren Umzug in ein anderes Land unternehmerischen Eifer unter Beweis gestellt.

\section{Hintergrund}

Die Hintergründe der Zuwanderer sind hochgradig verschieden. Einige von ihnen kommen als Flüchtlinge, die vor Krieg und Unterdrückung fliehen. Die Migranten in dieser Gruppe sind in den zahlreichen Phasen, die sie auf dem Weg vom Asylbewerber zum Flüchtling bis zum Erwerb der Staatsbürgerschaft durchlaufen müssen, nicht gut mit ihren Rechten in Zusammenhang mit Arbeit vertraut. Es besteht eine natürliche Zurückhaltung, ihren Status aufs Spiel zu setzen, indem sie sich an eine öffentliche Verwaltung wenden. Andere kommen aus wirtschaftlichen Gründen oder durch familiäre Verbindungen, sei es als Teil einer bestehenden Familie, durch eine arrangierte Heirat oder durch andere Vereinbarungen. Diese höchst heterogene Gruppe stellt die öffentliche Verwaltung, und insbesondere Unternehmensförderungsdienste, vor eine Herausforderung. Die meisten Unternehmensberater wissen wenig über Einwanderungsfragen, Arbeitsgenehmigungen und mit der sozialen Integration verbundene 
Herausforderungen. Dies beeinträchtigt ihre Fähigkeit, zugewanderten Unternehmern geeignete Unterstützung zu leisten. Unternehmensberater müssen für diese Fragen stärker sensibilisiert werden und einigermaßen über andere Agenturen, die in diesen Bereichen tätig sind, Bescheid wissen.

Außerdem haben Zuwanderer in der Regel Schwierigkeiten, sich in dem institutionellen und Regelungsumfeld ihres neuen Landes zurechtzufinden, weil es sich von dem in ihrem Heimatland unterscheidet. Beispiele hierfür sind unter anderem das Wissen, wie man eine Unterkunft findet, zu verstehen, wie man Gesundheitsdienstleistungen in Anspruch nehmen kann, welche öffentliche Verwaltung welche Funktionen wahrnimmt sowie andere Fragen, die sich im Alltag ergeben. Selbst Bürger der Europäischen Union aus den neuen Mitgliedstaaten der Europäischen Union machen die Erfahrung, dass viele Annahmen aus ihrem Heimatland nicht auf andere Mitgliedstaaten übertragbar sind.

Im Ergebnis setzen Zuwanderer auf den Rat von Freunden und Familie (die sich unter Umständen in einem anderen Land aufhalten). Diese Ratschläge von Familie und Freunden können falsch sein, was zu Fehlern führt. Diese Fehltritte setzen dem Selbstwertgefühl und dem Selbstvertrauen des Zuwanderers zu und führen zu Depression und Hoffnungslosigkeit.

Auf der Seite der Bereitstellung der Dienste sieht es so aus, dass für die öffentlichen Verwaltungen in Dänemark nur wenige ethnische Minderheiten tätig sind. In größeren Städten beginnt sich dies allerdings zu ändern. Dies sorgt dafür, dass die öffentlichen Verwaltungen an Attraktivität für Zuwanderer verlieren, denn Letztere tun sich schwer damit, Vertrauen zu den an vorderster Linie tätigen Mitarbeitern zu fassen. In einigen Fällen ist auch die Sprache ein Hindernis. Der Mangel an ausreichenden Dolmetschdiensten in vielen öffentlichen Verwaltungen bedeutet, dass Interaktionen mit Beamten sowohl für den Zuwanderer als auch für den Beamten häufig stockend, angespannt und frustrierend sind. Es passiert nicht selten, dass letzten Endes die Kinder der Familie bei den Terminen mit Beamten dolmetschen; doch auch wenn die Kinder genug Dänisch gelernt haben, um zu kommunizieren, kennen sie häufig die Fachbegriffe nicht und tragen zusätzlich zu Missverständnissen und Verwirrung bei. Das Problem verschärft sich weiter, wenn persönliche Belange der Eltern besprochen werden, zum Beispiel Geld, Gesundheit und die ehelichen Beziehungen.

Neben der Sprache stellt auch das Vertrauen die Kommunikation vor eine Herausforderung. Die Frage des Respekts hängt häufig mit der Sprache zusammen, doch gibt es unter Umständen andere Vorurteile, die stärker eingefahren sind. Migranten reagieren sehr sensibel auf Respektlosigkeit. Allgemein gibt es die Tendenz, Menschen, die nicht die eigene Landessprache sprechen, als unintelligent zu betrachten. Doch das Problem ist meistens nicht die Intelligenz, sondern die Verständigung. Die Mehrheit der Zuwanderer kommt ohne Dänischkenntnisse nach Dänemark. Während Kinder die Sprache häufig dank des völligen Eintauchens in der Schule innerhalb von einigen Monaten lernen, ist dies für Erwachsene viel schwieriger. Manche von ihnen sprechen nur wenig Dänisch, obwohl sie schon seit Jahren in Dänemark leben.

Einfühlungsvermögen ist für den Aufbau von Vertrauen in einer Beziehung von entscheidender Bedeutung. So sind zum Beispiele viele Zuwanderer zurückhaltend, wenn es darum geht, Fragen zu ihren finanziellen Mitteln ehrlich zu beantworten, denn sie wissen häufig nicht genau, wie das Steuersystem funktioniert, und fürchten, etwas falsch gemacht zu haben. Dies kann jedoch bei einem potenziellen Geschäftsplan ein großer Stolperstein sein, weil der Berater so nicht beurteilen kann, welche Ressourcen wirklich für eine Unternehmensgründung vorhanden sind. Die Finanzkanäle in Diaspora-Gemeinschaften sind häufig ziemlich komplex. Menschen einer Diaspora haben unter Umständen Verbindungen zu anderen Menschen in einer Reihe von Ländern, bei denen sie um Geld bitten können. Viele Flüchtlinge verlassen ihr Land mit leeren Händen, können aber mit der Zeit Verbindungen und Ressourcen aufbauen. Ein sachkundiger Betreuer versteht, wie heikel diese Aspekte sind, und ist in der Lage, Fragen zu Finanzen so zu stellen, dass er eine ehrlichere Antwort erhält.

Die verringerte Wirtschaftsleistung in vielen Zuwanderergemeinschaften verstärkt die Probleme bei der Bereitstellung von Diensten und das fehlende Einfühlungsvermögen und Vertrauen. Zuwanderergemeinschaften weisen häufig im Vergleich zur Bevölkerung des Gastlandes niedrigere Beschäftigungsquoten und eine niedrigere Aktivität auf, wobei dies zwischen den Gruppen untereinander und den Geschlechtern recht unterschiedlich ausfällt. Dies trifft besonders auf Frauen zu, vor allem auf Frauen mit muslimischem Hintergrund in Dänemark. Die oben erwähnten Wirtschaftszuwanderer siedeln häufig wegen einer bestimmten Stelle in einer Fabrik um. Endet diese Stelle aus irgendwelchen Gründen, 
sind sie unter Umständen nicht in der Lage, einen gleichwertigen Arbeitsplatz zu finden, und beginnen, aus der Not heraus über eine selbstständige Erwerbstätigkeit nachzudenken.

\section{Aktivitäten}

Das Projekt stützt sich auf zwei Hauptebenen: Informationssitzungen in Gruppen und persönliches Coaching. Informationssitzungen werden das ganze Jahr über angeboten und bieten Informationen zur Unternehmensgründung, zur selbstständigen Erwerbstätigkeit und zu den verfügbaren Unterstützungsleistungen. Mit den Informationssitzungen wird außerdem Werbung für das intensivere persönliche Coaching gemacht. Beim Coaching liegt der Schwerpunkt auf der Ermittlung der Bereitschaft der potenziellen Unternehmer für die Unternehmensgründung sowie auf der Leistung von Hilfestellung, damit möglichst viele von ihnen Erfolg haben. Das Projekt konzentriert sich auf ethnische Minderheiten in der Stadt. Anfangs waren die Empfänger dieser Unterstützung zum größten Teil türkischer Herkunft, inzwischen stammen die Teilnehmer jedoch aus den unterschiedlichsten Gebieten, unter anderem aus Nordafrika, vom Horn von Afrika sowie aus Zentral- und Osteuropa.

Das in Vejle eingesetzte Modell setzt auf eine persönliche Unterstützung in Verbindung mit etwa sechs Treffen pro Jahr, die der Kontaktaufnahme zu lokalen Verbänden gewidmet sind. Die individuelle CoachingKomponente beginnt mit einem Aufnahmegespräch, das bis zu zwei Stunden dauert. Somit ist es viel länger als das typische 30-minütige Gespräch, das in allgemeinen Unternehmensförderungsorganisationen angeboten wird. Der Betreuer verfolgt dabei das Ziel, detaillierte Informationen über die Situation seines Klienten zu erlangen. In vielen Fällen hat der Klient bereits ein Unternehmen im kleinen Stil gegründet und wurde dabei über familiäre Beziehungen, vielfach von außerhalb Dänemarks, unterstützt.

Der Betreuer hat die Funktion, professionelle Beratung zu den Rechts- und Sozialnormen im neuen Land anzubieten und die sozialen Netzwerke der ethnischen Minderheiten aufzubauen und zu stärken. Dies trägt dazu bei, die Rolle des Unternehmensberaters weg von der Familie und von Freunden hin zu professionellen Betreuern und Beratern zu verlagern, um für eine wirksamere Beratung zu sorgen. Die Beratung erfolgt im Rahmen von informellen und flexiblen Abmachungen. Zunächst liegt das Hauptaugenmerk auf dem Geschäftsplan: Die zentralen Elemente der Geschäftsidee sollen ermittelt und weiterentwickelt werden, unter anderem die Möglichkeit, der Markt, der Finanzierungsbedarf und die Kompetenzen des Unternehmers. Auf der Grundlage dieser Gespräche vermittelt der Betreuer die Teilnehmer an formelle Geschäfts- und Unternehmerschulungen weiter, die von den Regionen und Kommunen angeboten werden.

Im Anschluss an die Unternehmensgründung können die Teilnehmer weiterhin Coaching und Mentoring in Anspruch nehmen, um ihr Unternehmen weiterzuentwickeln und wachsen zu lassen.

Der Ansatz des Projekts stützt sich auf Respekt und Verständnis. Bei dem in Vejle eingesetzten Modell verfolgt der Betreuer einen ganzheitlichen Ansatz, um festzustellen, ob andere, mit der Beschäftigung zusammenhängende Probleme bestehen, die einem Erfolg des Unternehmens im Wege stehen. So kann zum Beispiel ein schlechter Gesundheitszustand ein Hindernis für die Unternehmensgründung darstellen. Dies trifft insbesondere auf diejenigen zu, die als Flüchtlinge ins Land gekommen sind und in ihrem Herkunftsland schwerwiegende Traumata erlebt haben. In einigen ethnischen Gemeinschaften stehen auch Frauen vor speziellen Hindernissen in Bezug auf das Unternehmertum, vor allem wenn Religion oder Kultur ihnen eine Rolle vorschreibt, die sich auf das häusliche Leben konzentriert. 
Dieses Projekt startete in der Kommune Vejle, doch inzwischen werden Projekte in ganz Dänemark durchgeführt.

\section{Textfeld 7.1. Profil eines Teilnehmers von „Geschäftscoaching für ethnische Minderheiten" Maria, Psychologin}

Ihre Idee bestand darin, Waren aus Polen zu importieren. Eine weitere Hinterfragung ergab, dass es die Idee ihres Ehemannes war. Da dieser jedoch kein Dänisch sprach, schlug Maria dieses Geschäft vor. Muhsin fragte sie, ob sie in fünf bis zehn Jahren als Psychologin tätig sein oder das Importgeschäft betreiben wolle. Er riet ihr, das zu tun, was sie glücklich macht. Sie erwiderte: „Sind Sie hier der Psychologe oder bin ich es?"

\section{Textfeld 7.2. Profil eines Teilnehmers von „Geschäftscoaching für ethnische Minderheiten": ein geflüichteter Schuster}

Einem geflüchteten Schuster aus dem Irak sagte der dänische Unternehmensberater, den der Schuster aufgesucht hatte, dass Schuhe in Dänemark in Fabriken hergestellt würden und er nicht als Schuster arbeiten könne. Er fand jedoch eine Stelle als Orthopädie-Schuhmacher und kaufte anschließend die Firma mit der Unterstützung eines Betreuers. Dieses Unternehmen beschäftigt nun fünf Menschen. Die Rolle des Betreuers bestand darin, dem Unternehmer dabei zu helfen, seine Fähigkeiten in einem anderen Kontext anzuwenden und Möglichkeiten zu erkennen.

\section{Herausforderungen}

Die größte Herausforderung für das Projekt ist die Sicherung einer finanziellen Unterstützung. In den letzten beiden Jahren (2012-2014) wurde das Projekt im Rahmen des nationalen Programms durch den Europäischen Sozialfonds unterstützt. Zuvor war die Stadtverwaltung nicht bereit, jemanden für die Betreuung von ethnischen Minderheiten zu bezahlten, sondern zog es vor, dass diese am allgemeinen Unternehmensförderungssystem teilnahmen. Allerdings funktionierte das allgemeine System nicht gut für die ethnischen Minderheiten, was zu einer fehlenden Nachfrage seitens der Unternehmer aus ethnischen Minderheiten führte. Der Betreuer hat diese Dynamik erfolgreich geändert, indem er sich an die Gemeinschaften wandte und unter Beweis stellte, dass diese Ergänzung zu den allgemeinen Diensten funktionieren kann, ohne dass ein Parallelsystem geschaffen wird.

\section{Auswirkungen}

Dieses kostengünstige Projekt erzielt gute Ergebnisse angesichts der dafür ausgegebenen Gelder. Es hat mehrere Auszeichnungen gewonnen, unter anderem den Europäischen Unternehmerpreis im Jahr 2007. Das Programm hat mehr als 250 Unternehmer mit jeweils 2000 EUR gefördert. Schätzungen zufolge gründen rund $50 \%$ derjenigen, die betreut wurden, in der Folge ein Unternehmen. Das Programm selbst gibt an, dass beinahe alle Unternehmen der Unternehmer, die ein Coaching in Anspruch nehmen, mindestens ein Jahr lang überleben.

\section{Voraussetzungen für die Übertragung}

Dieses kostengünstige Coaching-Modell kann in einfacher Weise für verschiedene Zielgruppen und/oder Regionen angepasst werden. Die zentralen Erfolgsfaktoren für die Übertragung der Erfahrung sind folgende:

1. Einsatz von Beratern aus der Gemeinschaft des Klienten. Der Einsatz von Unternehmensberatern und Betreuern, die zur Klientengruppe gehören, verbessert die Fähigkeit, potenzielle Klienten für das Unterstützungsangebot anzuziehen. So kann außerdem schneller Vertrauen zwischen dem Betreuer und dem Klienten aufgebaut werden, da beide mit höherer Wahrscheinlichkeit dieselben 
kulturellen Werte und Einstellungen teilen. Die Beratung und das Coaching gewinnen an Relevanz, da der Betreuer die Herausforderungen, mit denen der Klient konfrontiert ist, eher versteht.

2. Einsatz von professionellen Beratern. Ziel dieses Projekts ist die Verbesserung der Qualität der Beratung, die die Unternehmer erhalten. Untersuchungen zeigen, dass Unternehmer aus ethnischen Minderheiten die Nutzung öffentlicher Wirtschaftsförderungsdienste meiden und sich stattdessen stark auf Familie und Freunde verlassen (z. B. Ram und Smallbone, 2003). Der Einsatz eines professionellen Beraters verbessert die Qualität der bereitgestellten Unternehmensberatung.

3. Die Entwicklung persönlicher Beziehungen anstreben. In diesem Projekt setzt man auf einen persönlichen Ansatz, der durch einen persönlichen Betreuer entwickelt und verfeinert wurde. Die persönlichen Beziehungen trugen wesentlich dazu bei, die Klienten zu motivieren und ein Vertrauensverhältnis aufzubauen.

4. Sicherstellung einer flexiblen Beziehung. Wird Flexibilität bei der Häufigkeit, der Dauer und dem Ort der Treffen eingeräumt, sorgt dies für einen leichteren Zugang zur Unterstützung und erhöht die Chancen, dass das Anbot in der Klientengruppe in Anspruch genommen wird. Dies gilt insbesondere für Unternehmer, die neben der Führung eines Unternehmens familiäre Betreuungsaufgaben wahrnehmen müssen.

\section{Literatur}

Ram, M. und D. Smallbone (2003), „Policies to Support Ethnic Minority Enterprise: the English Experience“, Entrepreneurship and Regional Development, Bd. 15, Nr. 2, S. 151-166. 


\section{Beihilfe zur Unternehmensgründung, Finnland}

Diese Fallstudie stellt ein Beispiel für ein Programm zur Überbrückungshilfe vor, das Arbeitslose bei der Unternehmensgründung unterstützt. Sie enthält eine Beschreibung der Ziele der Beihilfe zur Unternehmensgründung und legt die Hintergründe dar. Außerdem werden die Funktionsweise sowie die Auswirkungen des Programms beschrieben. Ferner erörtert die Fallstudie die Herausforderungen des Programms und die Voraussetzungen für seine Übertragung auf einen anderen Kontext.

\section{Ziele}

Das Ziel der Beihilfe zur Unternehmensgründung ist die Stärkung neuer Unternehmensgründungen und die Förderung der Beschäftigung unter den Arbeitslosen. Außerdem zielt das Programm darauf ab, Einzelne zur Unternehmensgründung zu ermutigen und ihr Wachstum und ihre Beschäftigung zu fördern, um so die Überlebensraten neuer Unternehmensgründungen aufrechtzuerhalten und zu verbessern (Lehto und Stenholm, 2001). Die Beihilfe bietet neuen Unternehmern ein sicheres Einkommen, um während des Unternehmensstarts und der anfänglichen Unternehmensentwicklung einen Beitrag zur Sicherung des Lebensunterhalts des Unternehmers zu leisten. Sie wird in der Regel sechs Monate lang geleistet, kann aber zweimal verlängert werden. Die Höchstlaufzeit beträgt 18 Monate.

\section{Hintergrund}

In den 1980er-Jahren wurde ein landesweites Beihilfesystem für Unternehmensgründungen ins Leben gerufen. Während der schweren Rezession Anfang der 1990er-Jahre wurde es weithin genutzt, um die selbstständige Erwerbstätigkeit und das Unternehmertum unter den Arbeitslosen zu fördern, als andere Beschäftigungsmöglichkeiten rar waren. Im Verlauf der letzten Jahre haben sich die Arbeitsmärkte verändert, und atypische Beschäftigungsformen haben im Vergleich zu StandardArbeitsverträgen an Bedeutung gewonnen (Lehto und Stenholm, 2001). Diese Tendenzen haben dazu geführt, dass das Unternehmertum und die selbstständige Erwerbstätigkeit tragfähigere und passendere Optionen werden, durch die Menschen ihren Lebensunterhalt verdienen können und Finnland seine Wettbewerbsfähigkeit verbessern kann. Allerdings ist eine der Herausforderungen, mit denen Arbeitslose in Bezug auf die Unternehmensgründung konfrontiert sind, die fehlende Finanzierung, um den Lebensunterhalt, die Betriebskosten und Investitionsausgaben zu decken.

\section{Aktivitäten}

Das Programm wurde ursprünglich 1984 als Pilotprojekt ins Leben gerufen, das 1988 im größeren Stil neu aufgelegt wurde. Es läuft bis heute. Das Programm richtete sich anfangs an Arbeitslose und 
diente der Unterstützung ihrer Unternehmensgründungen und ihrer Beschäftigung. Die Menschen, die Anspruch auf die Beihilfe zur Unternehmensgründung haben, müssen als arbeitslose Arbeitsuchende beim TE-Büro (Büro für Beschäftigung und wirtschaftliche Entwicklung) gemeldet sein. Allerdings kann die Beihilfe zur Unternehmensgründung seit 2008 auch Menschen gewährt werden, die nicht arbeitslos sind, sondern im Begriff sind, von einer abhängigen Beschäftigung, vom Studium oder von Heimarbeit in eine selbstständige Erwerbstätigkeit in Vollzeit überzugehen.

Die Beihilfe zur Unternehmensgründung besteht aus einer Grundbeihilfe, die 2014 bei 32,66 EUR pro Tag lag (und für fünf Tage die Woche auf der Grundlage der Tage, an denen der Antragsteller im Start-up tätig ist, ausgezahlt wird), und einer zusätzlichen Beihilfe, die bis zu $60 \%$ der Grundbeihilfe (d. h. 19,60 EUR pro Tag) beträgt und die für Ideen vergeben werden kann, bei denen man ein hohes Potenzial annimmt. Die Beihilfe zur Unternehmensgründung wird in der Regel für Zeiträume von je sechs Monaten gewährt, und zwar bis zu 18 Monate lang. Im Durchschnitt betrug die Beihilfe 2013 etwa 800 bis 850 EUR monatlich für 9,5 Monate. Sie wird monatlich ausbezahlt und gilt als steuerpflichtiges Einkommen.

Die Beihilfe zur Unternehmensgründung wird vom TE-Büro bereitgestellt. Die Hauptkriterien für die Beihilfe sind folgende (Ministerium für Beschäftigung und Wirtschaft, 2014):

- in Vollzeit durchgeführte selbstständige Erwerbstätigkeit;

- ausreichend Fähigkeiten und Kompetenzen für das geplante Unternehmen;

- Potenzial für ein nachhaltiges, gewinnbringendes Unternehmen;

- Notwendigkeit des Antragstellers, seinen Lebensunterhalt zu verdienen;

- das Unternehmen wird erst nach Gewährung der Beihilfe gegründet.

Die Antragsteller sind verpflichtet, in ihrem Antrag in Kürze Folgendes vorzustellen: persönliche Arbeits- und Unternehmererfahrung; betriebswirtschaftliche und auf das Unternehmertum bezogene Bildung und Ausbildung; Name des geplanten Unternehmens; Rechtsform des geplanten Unternehmens; vorgeschlagene Eigentumsstruktur; Geschäftsidee (einschließlich Stärken und Schwächen); Einzelheiten zur vorgeschlagenen Tätigkeit (z. B. Räumlichkeiten, Personalbedarf); Produktionsverfahren (z. B. Einrichtungen, Produktionsfaktoren); Kundenprofile (z. B. wer, Menge, regionale Abdeckung, Bedürfnisse); Marketing-Pläne (z. B. Mittel und Kosten); Vertriebsmethoden; Wettbewerbsanalyse (z. B. Wettbewerber und ihre Angebote, eigene Stärken im Wettbewerbsumfeld); im Vorfeld der Unternehmensgründung ergriffene Maßnahmen; Finanzierungsbedarf und -quellen (z. B. Investitionen, Umlaufkapital, Eigenkapital, Darlehen, Beihilfen); Gewinn- und Verlustrechnung (z. B. Umsatzprognose, Umsatz- und Kostenerwartungen); Organisation der Buchhaltung (z. B. Notwendigkeit einer fachlichen Beratung); sowie andere erhaltene Beihilfen. Bei der Beihilfe zur Unternehmensgründung handelt es sich um eine De-minimis-Beihilfe: Dem Antragsteller oder dem Unternehmen können höchstens 200000 EUR während des aktuellen und der zwei vorangehenden Steuerjahre gewährt werden. Der Beihilfeempfänger ist dafür verantwortlich, die Obergrenze für Deminimis-Beihilfen, die durch verschiedene öffentliche Organisationen (wie Finnvera, einen öffentlichen Risikofinanzierer), Stadtverwaltungen und Provinzverbänden zur Verfügung gestellt werden, nicht zu übersteigen.

Das TE-Büro konsultiert bei der Evaluierung der Antragsteller externe Sachverständige. Deren Funktion besteht darin, allgemeine Informationen und Beratung zur Gründung eines Unternehmens zu bieten und zu ermitteln, ob das Geschäftskonzept tragfähig und wettbewerbsfähig ist. Bei seiner Entscheidung berücksichtigt das TE-Büro außerdem die Wettbewerbssituation und die Notwendigkeit des geplanten Unternehmens in der Region. Evaluierungsstudien zufolge funktioniert das Antragsverfahren relativ gut; 70\% der Beihilfeempfänger geben an, dass die Beantragung der Beihilfe einfach sei (Stenholm und Aaltonen, 2012; Stenholm, 2007; 2006).

Ein Kriterium für die Vergabe der Beihilfe ist, dass der Antragsteller über Erfahrungen oder eine Ausbildung im unternehmerischen Bereich verfügt. Können Antragsteller dies nicht vorweisen, können sie an kostenfreien Arbeitsmarktschulungen teilnehmen, die das TE-Büro anbietet. Diese umfassen auch Unternehmerkurse, die von verschiedenen Bildungseinrichtungen ausgerichtet werden. Die Kurse dauern üblicherweise vier bis acht Wochen. Jeder Dienstleister gestaltet das Schulungsangebot auf der 
Grundlage seines eigenen Fachwissens und der speziellen für den Kurs festgelegten Ziele. Während dieser Schulungen wird vom Antragsteller erwartet, dass er die Geschäftsidee und den Geschäftsplan weiterentwickelt und ein besseres Verständnis der Maßnahmen gewinnt, die bei der Vorbereitung der Unternehmensgründung zu ergreifen sind. Die Schulungen vermitteln den Teilnehmern außerdem Grundkenntnisse im Bereich Finanzen, Buchhaltung und Steuerfragen, Marketing, Risikomanagement und Personalmanagement. Ziel der Schulungen ist die Entwicklung der Bereiche, die den Antragsteller daran hindern könnten, ein tragfähiges Unternehmen zu führen.

\section{Projektfinanzierung}

Die Bestimmungen der Beihilfe zur Unternehmensgründung sind im Gesetz zur öffentlichen Beschäftigung und zu Unternehmensdienstleistungen und in einer Regierungsverordnung enthalten (Ministerium für Beschäftigung und Wirtschaft, 2014). Der Jahreshaushalt für Beihilfen zur Unternehmensgründung beläuft sich auf etwa 38 Millionen EUR. Das Programm erhielt zwischen 2010 und 2012 etwa 2 Millionen EUR jährlich aus dem Europäischen Sozialfonds.

\section{Herausforderungen}

Das Ministerium für Beschäftigung und Wirtschaft hat mehrere Studien über die Beihilfe zur Unternehmensgründung in Auftrag gegeben (Stenholm, 2013; Stenholm und Aaltonen, 2012; Stenholm, 2007; 2006), um mehr über ihre Auswirkungen und ihre Wirksamkeit zu erfahren.

Die Evaluierungen stellen durchweg fest, dass die Beurteilung, ob der Unternehmer die Beihilfe benötigt und wie die Beihilfe die Gründungsentscheidung und das Gründungsverfahren der Unternehmer beeinflusst, eine Herausforderung darstellt (Stenholm 2006; 2007; Stenholm und Aaltonen, 2012). Es ist schwierig, das Kriterium des „Bedarfs“ einerseits gegen die Voraussetzungen für ein gewinnbringendes Unternehmen andererseits abzuwägen. Ein weiterer Beleg für diesen Konflikt ergibt sich bei der Erörterung der potenziellen Sekundärmarkteffekte des Programms - rund die Hälfte der Unternehmensgründer waren der Ansicht, sie hätten ihr Unternehmen auch ohne diese Beihilfe gegründet (Stenholm und Aaltonen, 2012).

Allgemeiner gefasst steht das Programm vor der Herausforderung, Richtlinien für die Sachverständigen vorzulegen, die die Unternehmer und das Potenzial ihrer Unternehmen beurteilen. Das Antragsverfahren stützt sich zum Teil auf Stellungnahmen von Sachverständigen, die das Potenzial des geplanten Unternehmens beurteilen, und obwohl die Beamten, die die Beihilfeentscheidungen vorbereiten und fällen, mit der Qualität und dem Inhalt der Stellungnahmen relativ zufrieden waren, basierten diese Entscheidungen auf persönlichen Wahrnehmungen. In Bezug auf diese Entscheidungen muss ein einheitliches Vorgehen gewährleistet werden.

Die Evaluierungen deuten auch darauf hin, dass mehr Unterstützung und Beratung während des Gründungsprozesses für die Antragsteller hilfreich gewesen wäre (Stenholm, 2006). Es wurde vorgeschlagen, dass öffentliche Akteure stärker zusammenarbeiten sollten, um die Effizienz der Beihilfe zu erhöhen, da sie unter Umständen dieselben potenziellen Unternehmer bedienen und es einem ineffizienten Einsatz von Mitteln gleichkommt, dieselbe Geschäftsidee und denselben Unternehmer mehrmals zu prüfen (Stenholm und Aaltonen, 2012).

Die Beihilfe zur Unternehmensgründung ist ein allgemein bekanntes und gut etabliertes Instrument zur Unterstützung von Start-ups. Obwohl es keine besonderen Sensibilisierungskampagnen gibt, ist die Beihilfe auf Internetseiten des öffentlichen Sektors sichtbar, die für Unterstützungsdienste für diejenigen werben, die das Unternehmertum als eine Option für sich betrachten. Zusätzlich informieren Beamte des TE-Büros Arbeitslose auf Arbeitssuche über die Verfügbarkeit der Beihilfe. Allerdings kennen die Beihilfeempfänger häufig einige der damit verbundenen Unterstützungsdienste und die Möglichkeit der Verlängerung der Beihilfen nicht. Daher könnten die TE-Büros besser für entsprechende Informationen werben (Stenholm und Aaltonen, 2012).

Und schließlich waren die Unternehmer, die die Beihilfe erhielten, unzufrieden mit der Höhe und Dauer der Beihilfe. Das Ministerium erkennt an, dass die Beihilfe nicht ausreicht, um das Unternehmen auf dem Markt zu etablieren, aber ihr Ziel ist es, den Antragsteller bei seinen Lebenshaltungskosten zu unterstützen (Stenholm, 2006). Am wenigsten zufrieden waren Unternehmer, die die Beihilfe 
zur Unternehmensgründung zusätzlich zu anderen öffentlichen Mitteln in Anspruch nahmen, die vor der Unternehmensgründung arbeitslos waren, die älter als 40 Jahre alt waren und die bereits unternehmerische Erfahrungen hatten (Stenholm und Aaltonen, 2013).

\section{Auswirkungen}

Die Auswirkungen der Beihilfe zur Unternehmensgründung für viele Einzelunternehmer gelten als positiv. Ausgehend von einer kürzlich durchgeführten Evaluierung hat sie es Unternehmern ermöglicht, ihren Gründungsprozess zu beschleunigen, und rund die Hälfte der Befragten betrachtete die Beihilfe als notwendig für ihren Lebensunterhalt. Außerdem empfand fast die Hälfte der Unternehmer die Beihilfe als Ermutigung durch den Staat - die Beihilfe war auf gesellschaftlicher Ebene ein positives Signal für das Unternehmertum. Die größten Auswirkungen verzeichnete man unter den Frauen und unter denjenigen, die bereits bescheidene Erfahrungen mit dem Unternehmertum oder in der Industrie hatten, was darauf hindeutet, dass die Beihilfe möglicherweise den Mangel an persönlichen Netzwerken ausgleicht (Stenholm und Aaltonen, 2012).

Die Beihilfe hat zudem positive Auswirkungen auf die Überlebensraten der Unternehmen: So waren zum Beispiel 38\% der 2005 gegründeten Unternehmen im Jahr 2012 immer noch in Betrieb. Für die 2006 bzw. 2007 gegründeten Unternehmen lag die Rate bei 41\% bzw. 61\%. Allerdings zeigen die Längsschnittstudien, dass die Überlebensrate bei den Unternehmen, die von nicht arbeitslosen Menschen gegründet wurden, höher liegt als bei den von Arbeitslosen gegründeten Unternehmen. Ferner deuten die Ergebnisse auf ähnliche Unterschiede in der Entwicklung der Umsätze und der Beschäftigung der untersuchten Unternehmen hin. Diejenigen, die ihr Vorhaben während ihrer Arbeitslosigkeit starteten, schätzen ihre Umsätze und ihre Mitarbeiterzahlen niedriger ein als ihre nicht arbeitslosen Pendants. Im Allgemeinen verzeichnete man höhere Überlebensraten bei denen mit höherem Bildungsstand, mehr Sozialkapital und bei denen, die nicht aus der Arbeitslosigkeit heraus gründeten (Stenholm, 2013).

\section{Voraussetzungen für die Übertragung}

Das Programm „Beihilfe zur Unternehmensgründung“ läuft in Finnland seit etwa 30 Jahren und die Ergebnisse mehrerer Evaluierungen werden jeweils bei der Erneuerung des Programms berücksichtigt. Eine Reihe von ermittelten Erfolgsfaktoren dürfte für die Übertragung der Erfahrung auf andere Kontexte von zentraler Bedeutung sein.

1. Bereitstellung von Mitteln zur Sicherung des Lebensunterhalts. Das Programm ist äußerst geeignet für Arbeitslose, aber auch für andere Gruppen relevant, da es den Einzelnen während der ersten Monate des neuen Unternehmens bei der Sicherung des Lebensunterhalts unterstützt. Aufgrund der bescheidenen Geldsumme, die gewährt wird, und des relativ kurzen Zeitraums, in dem diese bezogen werden kann (bis zu 18 Monate), reicht die Beihilfe nicht zur Finanzierung eines neu gegründeten Unternehmens und zur Sicherung seines Überlebens aus, doch erhöht sie den Anreiz, sich für eine Unternehmensgründung zu entscheiden.

2. Unterstützung des Zugangs zu Unternehmensfinanzierung. Da die Beihilfe zur Unterstützung des Unternehmers, nicht des Unternehmens, bereitgestellt wird, schließt es nicht die Lücken im Startkapital für das Unternehmen. Es müssen andere Finanzierungsprogramme oder Bankdarlehen ermöglicht werden, die diese Beihilfe ergänzen können. Rund die Hälfte der Beihilfeempfänger beantragte auch eine Finanzierung aus anderen Quellen, hauptsächlich bei Banken und bei Finnvera Plc, dem staatlichen Risikofinanzierer. Der Finanzierungsbedarf ist bei diesen Start-ups in der Regel relativ bescheiden, da die Unternehmen, die mit der Unterstützung der Beihilfe zur Unternehmensgründung gegründet werden, häufig im Dienstleistungssektor oder im Einzelhandel tätig sind. 
3. Definition klarer Auswahlkriterien. Einerseits könnte die Beihilfe Evaluierungen zufolge für diejenigen wichtiger sein, die aus der Arbeitslosigkeit heraus ein Unternehmen gründen. Andererseits haben die Unternehmen, deren Gründer von einer abhängigen in eine selbstständige Erwerbstätigkeit gewechselt sind, höhere Umsätze und ein größeres Potenzial für die Arbeitsplatzbeschaffung. Auch ihre Überlebensraten sind höher. Daher sollten bei der Übertragung dieser Erfahrung auf einen anderen Kontext die Ziele (d. h. die sozialen gegenüber den wirtschaftlichen Zielen) klar definiert werden, damit eine geeignete Mischung aus Kandidaten ausgewählt wird und die Kosten aufgrund von Mitnahmeeffekten minimiert werden.

4. Einbindung des privaten Sektors in die Auswahl. Die Nutzung des Fachwissens des privaten Sektors im Auswahlverfahren kann zu besseren Ergebnissen führen, da diese Vertreter wahrscheinlich mehr Fachwissen bei der Auswertung von Geschäftsvorschlägen haben als die des öffentlichen Sektors. Es ist wichtig, Beihilfeempfänger auszuwählen, die eine realisierbare Geschäftsidee und reelle Erfolgsaussichten haben. Es könnte sogar nachteilig sein, denjenigen die Beihilfe zu gewähren, bei denen ein Scheitern wahrscheinlich ist, denn diese erleiden unter Umständen persönliche finanzielle Verluste und ihr Selbstvertrauen nimmt ab. In Bezug auf die arbeitslose Bevölkerung kann dies die Wahrscheinlichkeit der sozialen Ausgrenzung und der Verarmung erhöhen.

5. Angemessene Erwartungen. Die Beihilfe zur Unternehmensgründung wird wahrscheinlich keine hochgradig innovativen oder wachstumsorientierten Unternehmen hervorbringen. Dies mindert jedoch nicht den Wert eines solchen Programms, das zu einer großen Anzahl neuer Unternehmen und neuer Beschäftigung beigetragen hat, sowohl durch die selbstständige Erwerbstätigkeit als auch durch die neuen Arbeitsplätze, die in den Start-ups geschaffen wurden.

6. Breit angelegte Werbung. Die Beihilfe zur Unternehmensgründung konnte eine durchgehend hohe Inanspruchnahme erreichen, indem weithin auf vielen öffentlichen Websites und durch öffentliche Arbeitsverwaltungen für das Programm geworben wurde.

\section{Literatur}

Lehto, J. und P. Stenholm (2001), „Yrittäjyyskoulutuksen vaikuttavuus ja starttirahayritysten menestyminen Varsinais-Suomessa“, Turun kauppakorkeakoulu, Yritystoiminnan tutkimus- ja koulutuskeskuksen julkaisuja B5/2001.

Ministerium für Beschäftigung und Wirtschaft (2014), „Starttiraha - aloittavan yrittäjän tuki“, verfügbar unter: www.te-services.fi/te/en/employers/for_entrepreneurs/services_new_entrepreneurs/startup_grant/ index.html (Zugriff am 12. Mai 2014).

Stenholm, P. (2007), „Start-up grant as a key to entrepreneurship. New experiences from the start-up pilot 2005-2007“, Forschungsstudie des Arbeitsministeriums 332.

Stenholm, P. (2006), „With help of the start-up grant to entrepreneurship - Experiences from the start-up grant trial“, Forschungsstudie des Arbeitsministeriums 320.

Stenholm, P. und S. Aaltonen (2012), „Promoting entrepreneurship through start-up grants. Impacts and functionality“, Ministerium für Beschäftigung und Wirtschaft. 


\section{Adie Mikrofranchising, Frankreich}

Diese Fallstudie stellt ein neues, innovatives Modell zur Unterstützung von Arbeitslosen bei der Unternehmensgründung vor, bei dem ihnen ein sofort einsatzbereites Unternehmens-Franchise, begleitet von Schulungen, Beratung und anderen Unterstützungsleistungen, angeboten wird. Die Ziele dieses Ansatzes sowie sein Hintergrund werden vorgestellt, und es wird die Funktionsweise des Modells beschrieben. Ferner erörtert die Fallstudie die ersten erzielten Ergebnisse, die Herausforderungen bei der Einrichtung des Programms und die Voraussetzungen für seine Übertragung auf einen anderen Kontext.

\section{Ziele}

Die Mission von Adie ist die Finanzierung und Unterstützung von Arbeitslosen, die ein eigenes Unternehmen gründen möchten und kein gewöhnliches Bankdarlehen erhalten. Die Zielklienten von Adie sind Unternehmer mit niedrigem Einkommen, die keinen Zugang zu den gängigen Finanzdienstleistungen haben, namentlich Arbeitslose, Sozialhilfeempfänger und Menschen mit niedrigem Bildungsstand. Die wichtigsten Unterstützungsprogramme von Adie bieten Mikrokredite zum Marktkurs (bis zu 10000 EUR) und Hilfe bei der Beantragung nationaler und lokaler Beihilfen zur Unternehmensgründung und zinsfreier nachrangiger Kredite. Außerdem stellen die Mitarbeiter von Adie und die dort tätigen Freiwilligen den Klienten nach der Bewilligung von Darlehen Geschäftsberatung im Bereich Betriebswirtschaft, administrative Anforderungen und Marketing sowie Rechtsberatung zur Verfügung.

Adie hat kürzlich eine neue Initiative zur Unterstützung des Unternehmertums von Unternehmern mit niedrigen Einkommen eingeführt. Dazu erhalten diese Zugang zu Franchises und damit zu einer Geschäftsidee, einem Geschäftsmodell, der möglichen Unterstützung durch den Franchisegeber oder andere gleichgestellte Franchisenehmer und $\mathrm{zu}$ einer Marke. Das Ziel des FranchisingProjekts ist es, einen risikoärmeren Zugang zum unternehmerischen Prozess zu ermöglichen. Die Mikrofranchisenehmer sollen gefördert werden, indem ihnen ein Produkt oder eine Dienstleistung sowie ein sofort einsatzbereites, bereits erprobtes Geschäftsmodell zur Verfügung gestellt werden.

\section{Hintergrund}

Forschungsstudien des Nationalen Instituts für Statistik und Wirtschaftsstudien (INSEE) haben ergeben, dass im Wesentlichen zwei Hindernisse von einer Unternehmensgründung in Frankreich abschrecken: Schwierigkeiten bei der Einhaltung administrativer Vorgaben und die Befürchtung, sich nicht ausreichend Startkapital sichern zu können (INSEE, 2011). Diese Hindernisse sind für potenzielle Unternehmer aus benachteiligten Gruppen noch höher, da diese in der Regel über wenige Ersparnisse, ein niedriges Qualifikationsniveau und schwach entwickelte Netzwerke verfügen und es 
ihnen an unternehmerischen Erfahrungen mangelt. Ziel von Adie ist es, diese Hindernisse durch die Erleichterung des Zugangs zu Finanzierung und zu einer Reihe von Unterstützungsleistungen für die Unternehmensgründung anzugehen.

Adie rief seine Mikrofranchising-Initiative ins Leben, weil immer mehr Menschen in Zweigstellen der Vereinigung kamen, die bereit waren, ein eigenes Unternehmen zu gründen, aber keine tragfähige Geschäftsidee hatten. Traditionelles Franchising ist für Unternehmer mit niedrigem Einkommen nicht erschwinglich und bietet daher für diese Gruppe keinen möglichen Weg in das Unternehmertum.

\section{Aktivitäten}

Das Mikrofranchising-Programm unterscheidet sich von den traditionellen Unterstützungsprogrammen von Adie darin, dass die Klienten nicht ihre eigenen Projekte vorschlagen. Stattdessen führt Adie die Klienten in verschiedene Mikrofranchising-Netzwerke ein, um dort ein passendes Projekt für sie zu finden. Adie bietet den Mikrofranchisenehmern ein umfassendes Spektrum an integrierten Dienstleistungen, darunter eine persönliche Bedarfsanalyse, Unterstützung bei der Entwicklung eines Geschäftsplans, Unternehmensberatungsdienste, Schulungen und Finanzierung.

Der Begriff „Mikrofranchising“ lässt sich anhand der beiden Grundwörter erklären, aus denen sich der Begriff zusammensetzt: Mikro und Franchising. Das „Mikro“ in Mikrofranchising bezeichnet den sozialen Aspekt der Unterstützung der Armen am Fuße der wirtschaftlichen Pyramide. „Franchising“ bezeichnet die Systematisierung und Vervielfältigung von Unternehmen. Somit nutzt das Mikrofranchising-Programm den Vervielfältigungsprozess eines Franchise, wendet diesen jedoch zum Zwecke der Armutslinderung auf benachteiligte Menschen an. Dieses System unterscheidet sich in einem wichtigen Aspekt vom traditionellen Franchising: Es ist unwahrscheinlich, dass Mikrofranchisenehmer ihre Anteile an einen anderen Betreiber veräußern können. Was die Mikrofranchisenehmer „besitzen“, ist nicht das Recht an bestimmten Vermögenswerten, die geringfügig sind, sondern eine bestimmte Beziehung mit dem Mikrofranchisegeber. Abbildung 9.1. veranschaulicht den allgemeinen Rahmen.

\section{Abbildung 9.1. Adie-Mikrofranchising-Rahmen}

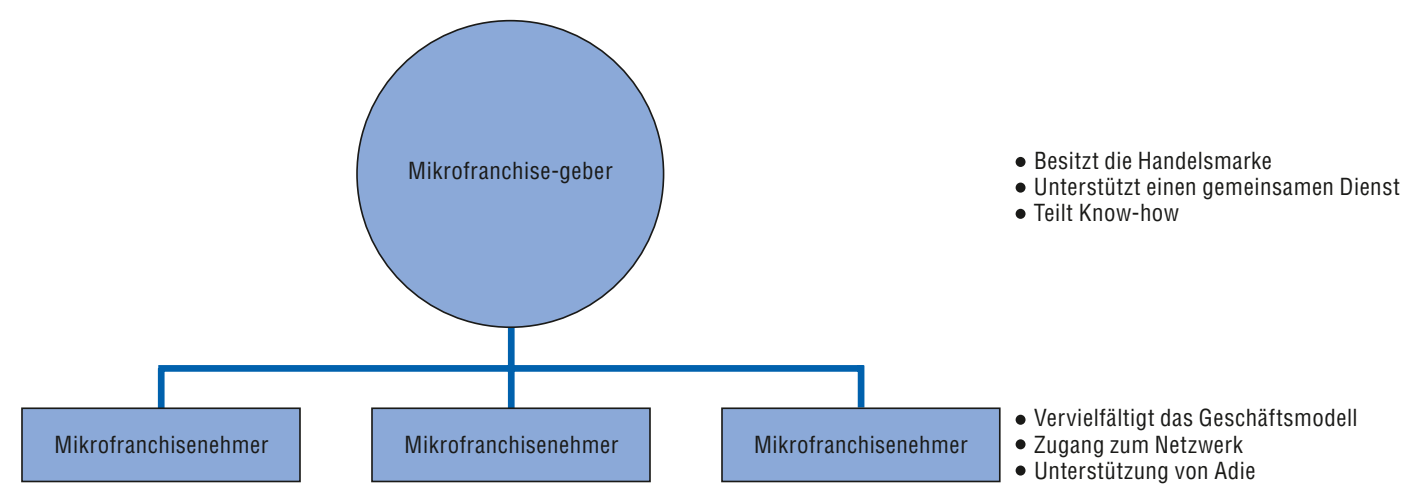

Die sofort einsatzbereiten Mikrofranchising-Geschäftsmodelle von Adie sind einfach, zugänglich und lassen sich leicht vervielfältigen. Die Mikrofranchisenehmer profitieren vom Zugang zu einer Handelsmarke, Know-how, gemeinsamen Diensten und der Unterstützung des Mikrofranchisegebers. Die Franchisegeber werden über die Beziehungen von Adie mit nationalen und internationalen Konzernen (z. B. Veolia, SAUR, L'Oréal) ausgewählt und entwickelt. Adie ist verantwortlich für die Identifizierung der verschiedenen Projekte und die Prüfung ihrer Robustheit und handelt die Franchising-Vereinbarungen aus. Bei Adie sind zwei engagierte Mitarbeiter in Vollzeit, ein Praktikant und zehn Freiwillige tätig. 
Um im Rahmen dieses Programms ein Franchiseunternehmen zu gründen, muss der Mikrofranchisenehmer das entsprechende Startkapital bereitstellen oder beschaffen. Dies beträgt in der Regel weniger als 10000 EUR und kann auch in Form eines Mikrokredits von Adie beschafft werden. Die Kandidaten werden durch Gespräche ausgewählt, in denen ihre Motivation und ihre Qualifikationen beurteilt werden. Die Franchisenehmer werden entweder direkt zur Teilnahme am Programm zugelassen, oder Adie schlägt den Kandidaten die Teilnahme an Schulungen zur Weiterentwicklung ihrer Kenntnisse vor.

Es bestehenVertragsbeziehungen zwischen dem Mikrofranchisegeber, dem Mikrofranchisenehmer und Adie. Die Mikrofranchisenehmer müssen dem Mikrofranchisegeber für das Recht, ihr Geschäftsmodell zu nutzen, eine kleine Lizenzgebühr zahlen. Die meisten Mikrofranchising-Projekte konzentrieren sich im Einzelhandel und im Dienstleistungssektor. Dazu gehören zum Beispiel Grafikdesigner, Anstreicher, Floristen, Lebensmittelhändler, Kosmetiker, Restaurants, Architekten, Landwirte und Fotografen. Es gibt zwei verschiedene Mikrofranchising-Modelle (Textfeld 9.1).

\section{Textfeld 9.1. Arten von Mikrofranchising bei Adie}

1. Partnerschaften mit Großkonzernen:

- Großes Arbeitsplatzbeschaffungspotenzial (das Ziel sind mindestens 200 Mikrofranchisenehmer pro Netzwerk).

- Beispiel: Das Mikrofranchising O2 Adie, ein Netzwerk aus Mikrofranchises im Bereich Gartenbau. Dieses 2011 ins Leben gerufene Netzwerk besteht derzeit aus 30 Gärtnern.

- Andere Projekte werden auf ihr Potenzial hin untersucht, unter anderem Verkäufer von Streetfood und Haushaltsleistungen wie Bügeln.

2. Partnerschaften mit Unternehmern:

- Mittleres Arbeitsplatzbeschaffungspotenzial (das Ziel sind etwa 50 Mikrofranchisenehmer pro Netzwerk).

- Beispiele: i) Chauffeur\&Go, ein kurzfristiger Chauffeurdienst, der 2010 startete. 2013 bestand dieses Netzwerk aus 90 Fahrern. Ziel ist es, das Netzwerk bis 2015 auf mindestens 130 Fahrer auszuweiten; ii) Mon Coursier de Quartier, ein 2015 ins Leben gerufener Fahrradtaxidienst, der Passagiere befördert und kleine Pakete liefert.

- Andere Fahrdienste werden auf ihr Potenzial als Mikrofranchise-Netzwerk hin untersucht.

Adie verwaltet die Mikrofranchise-Netzwerke über seine lokalen Agenturen. Adie bietet auch Finanzierungen an, die entweder durch seine Mikrokreditprogramme oder durch einen eigens dafür eingerichteten Mikrofranchise-Investmentfonds bereitgestellt werden. Mikrofranchisenehmer, die einen Mikrokredit benötigen, stellen einen Antrag im Rahmen der Standardverfahren für Mikrokredite. Dazu geht der Antrag an einen Kreditausschuss, der sich aus Mitarbeitern von Adie und freiwilligen Geschäftsführern, Buchhaltern und Bankern zusammensetzt. Der Investmentfonds Adie Microfranchise Solidaire Investissement hat den Auftrag, den Aufbau und die Entwicklung von MikrofranchiseNetzwerken durch den Erwerb von Kapitalanteilen an Mikrofranchise-Unternehmen zu unterstützen und zu finanzieren. Beide Finanzierungsinstrumente legen ihren Entscheidungen die Kapazität und Motivation der Mikrounternehmer sowie die Realisierbarkeit des Projekts zugrunde.

\section{Herausforderungen}

Damit das Mikrofranchising-Programm entworfen und umgesetzt werden konnte, mussten mehrere institutionelle und externe Hindernisse bewältigt werden. Das administrative Umfeld schaffte einige Herausforderungen, da komplexe einzelstaatliche Rechtsvorschriften soziale Innovationen nicht immer unterstützen. Zum Beispiel bestehen Auflagen für die Ausbildung und Zertifizierung von Fahrradreparateuren oder Heimlieferservices. Dies hat Auswirkungen für die Mikrofranchisenehmer, da für viele dieser gering qualifizierten Berufe Schulungen und eine Zertifizierung erforderlich sind. Adie schlägt Schulungen vor und steuert diese, den damit verbundenen Kosten- und Zeiteinsatz 
trägt jedoch der Mikrofranchisenehmer. Eines der Ziele des Programms ist die Bewältigung dieser Herausforderung, indem Geschäftsmodelle vorgeschlagen werden, für die keine Schulungen oder Zertifizierungen erforderlich sind.

Eine weitere Herausforderung des Programms ist die Auswahl geeigneter Klienten, die mit einiger Wahrscheinlichkeit mit Erfolg Mikrofranchises betreiben werden. Adie nutzt die Motivation und die Ausbildung als Prädiktoren für den Erfolg, aber die bisherige Erfahrung konnte die Auswahlkriterien nicht erfolgreich so verfeinern, dass ein Erfolg als Mikrofranchise-Betreiber besser vorhergesagt werden kann.

Bei der Weiterentwicklung des Programms durch Adie stößt man auf eine Reihe von strategischen Herausforderungen. Erstens stellt es eine Herausforderung dar, das Programm zu geringen Kosten auszuweiten. Das Programm stützt sich auf freiwillige Geschäftsexperten, die bei der Auswahl der Kandidaten und bei der Bereitstellung von Schulungen und Unterstützung mithelfen. Für eine Vergrößerung des Programmumfangs werden mehr Freiwillige und mehr Mitarbeiter, die diese Freiwilligen schulen, benötigt. Zweitens würden die Organisatoren gern die Verbindungen zwischen dem Mikrofranchising-Programm und anderen Unterstützungsangeboten von Adie wie Beratung und Schulungen verstärken. Drittens müssen die Verbindungen zwischen den Mikrofranchisenehmern und den Finanzinstituten verbessert werden, damit die Mikrofranchise-Netzwerke wachsen können.

\section{Auswirkungen}

Bis heute hat die Mikrofranchising-Initiative von Adie drei Mikrofranchise-Netzwerke entwickelt und mehr als 150 selbstständige Tätigkeiten geschaffen. Fünf weitere Mikrofranchise-Projekte werden derzeit untersucht oder befinden sich in der Pilotphase. Die Kosten für dieses Projekt belaufen sich auf rund 8000 EUR pro geschaffenen Arbeitsplatz und liegen damit erheblich unter den Gesamtkosten für die Arbeitslosenunterstützung eines Arbeitslosen (EMN, 2014).

Zusätzlich zur Schaffung von Arbeitsplätzen kann das Mikrofranchising-Modell die Arbeitsqualität für Geringverdiener verbessern. Im O2-Gärtnernetzwerk erreichen Mikrofranchisenehmer in der Regel nach drei Monaten Rentabilität und verdienen etwa das Anderthalbfache des Mindestlohns (EMN, 2014).

Das soziale Mikrofranchising von Adie basiert auf einem Geschäftsmodell, das sowohl auf der Ebene des Mikrofranchisegebers als auch auf der Ebene des Mikrofranchisenehmers rentabel ist. Diese Rentabilität auf zwei Ebenen soll die langfristige Nachhaltigkeit der Mikrofranchise-Netzwerke sichern. Während die Mikrofranchisenehmer in der Theorie sehr schnell ihre Kosten decken können müssen, erreichen die Mikrofranchisegeber in der Regel nach zwei bis drei Jahren Tätigkeit die Gewinnschwelle. Chauffeur\&Go, das erste Mikrofranchise-Netzwerk von Adie, startete 2010 und wurde 2013 rentabel.

\section{Voraussetzungen für die Übertragung}

Das Mikrofranchising-Programm von Adie wurde mit dem Ziel eingerichtet, sicherzustellen, dass sowohl das Geschäftsmodell des Mikrofranchisegebers als auch das des Mikrofranchisenehmers nachhaltig ist. Dieses zweistufige Ziel soll in jedem Netzwerk für langfristige Nachhaltigkeit sorgen. $\mathrm{Zu}$ den zentralen Faktoren, die bei der Vervielfältigung dieses Modells andernorts zu berücksichtigen sind, gehören unter anderem folgende:

1. Vereinfachung des unternehmerischen Prozesses. Eines der wesentlichen Merkmale des Mikrofranchising besteht darin, dass ein bewährtes Geschäftsmodell auf einen neuen Unternehmer übertragen wird, dem dabei Hilfe und Unterstützung geleistet wird. Dadurch entfällt die Notwendigkeit, dass neue Unternehmer „das Rad neu erfinden“. Viele der Vorteile des Mikrofranchising leiten sich aus der gegenseitigen Abhängigkeit zwischen Franchisegeber und Franchisenehmer ab. Die Franchisegeber möchten ihre Beziehungen zu ihren Mikrofranchisenehmern schützen, und das können sie am besten, wenn Letztere Erfolg haben. Die Mikrofranchisenehmer wiederum verdanken ihr gesamtes Geschäft der besonderen Beziehung mit dem Franchisegeber. Diese Verbindung gibt den Franchisegebern die Sicherheit, in Franchisenehmer zu investieren, die zuversichtlich sind, dass beide Gewinne erzielen werden. 
2. Sicherstellung des Zugangs der Mikrofranchisenehmer zu Wirtschaftsförderungsdiensten. Adie bietet dem Mikrofranchisenehmer ein gewisses Maß an Unterstützung, welche die Unterstützung, die direkt durch den Mikrofranchisegeber geleistet wird, ergänzt. Schulungen und eine individuelle Unterstützung vermitteln dem neuen Unternehmer ausreichend unternehmerische Fähigkeiten und Ermutigung und erhöhen so seine Erfolgschancen.

3. Nutzung des Fachwissens des Mikrofranchisegebers. Der Franchisegeber nimmt bei diesem Programm eine entscheidende Rolle ein. Es ist wichtig, dass sein Geschäftsmodell vereinfacht wird und übertragbar ist. Er muss auch bereit sein, den Mikrofranchisenehmer mit Schulungen, Unterstützung und Qualitätskontrolle zu fördern.

4. Aufbau eines kleinen Stabs von sachkundigen und engagierten Mitarbeitern. Die Programmmitarbeiter sollten nicht nur das Projekt verwalten können, sondern sowohl die Mikrofranchisenehmer als auch die Mikrofranchisegeber aktiv unterstützen. Es ist wichtig, dass die zentralen Mitarbeiter über unternehmerische Kenntnisse und Erfahrung verfügen und im Bereich Unternehmerausbildung, Finanzierung und in damit zusammenhängenden Aspekten sowie im Coaching geschult sind, technische Unterstützung leisten zu können. Auch ist es wichtig, dass sie mit Begeisterung Werbung für das Programm machen, Beziehungen zu potenziellen Mikrofranchisegebern entwickeln und sich um das Tagesgeschäft wie etwa die Durchführung von Aufnahmegesprächen kümmern.

5. Entwicklung geeigneter Auswahlkriterien. Um zum Erfolg des Mikrofranchising beizutragen, ist es wichtig, Mikrofranchise-Netzwerke um Geschäftsmodelle herum aufzubauen, die Wachstumspotenzial haben. Wichtige Überlegungen hierbei sind i) die Größe, Zugänglichkeit und Nachhaltigkeit des Marktes; ii) die Wirtschaftlichkeit des Geschäftsmodells auf Ebene des Franchisegebers und des Franchisenehmers; iii) das Fachwissen und die Unterstützung der Mikrofranchisegeber; und iv) Transparenz und Ausgewogenheit in der Zusammenarbeit zwischen den drei Partnern, sprich dem Organisator, dem Mikrofranchisegeber und dem Mikrofranchisenehmer.

\section{Literatur}

Europäisches Mikrofinanzierungsnetz (2014), „EMN Good Practices - ADIE - 2014“, verfügbar unter: www.european-microfinance.org/docs/emn_publications/good_practices/13.\%20Good\%20Practices_ADIE_ Adie\%20Social\%20Microfranchising\%20Initiative.pdf.

INSEE (2012), „Créations et créateurs d'entreprises - Première interrogation 2010, profil du créateur“, INSEE Résultats, Nr. 58, Februar. 


\section{Neuer Gründungszuschuss, Deutschland}

Diese Fallstudie stellt ein nationales Programm zur Überbrückungshilfe vor, das Arbeitslose bei der Unternehmensgründung unterstützt. Es werden die Ziele dieses Ansatzes, sein Hintergrund sowie die verschiedenen Versionen der Zuwendung, die in den letzten Jahren gewährt wurden, beschrieben. Außerdem werden die bei der Gestaltung und Umsetzung des Programms sichtbar werdenden Herausforderungen und die Voraussetzungen für die Übertragung dieser Erfahrung auf einen anderen Kontext erörtert.

\section{Ziele}

Das Ziel des neuen Gründungszuschusses besteht darin, Arbeitslose bei der Gründung eines Unternehmens finanziell zu unterstützen. Neben den üblichen Strategien bei der Stellensuche kann dies eine zusätzliche Option bieten, um der Arbeitslosigkeit zu entkommen. Außerdem schaffen einige der bezuschussten Unternehmen möglicherweise in der Zukunft neue Arbeitsplätze. Das Programm begann 1986 als Überbrückungsgeld und wurde 2003, 2006 und 2011 angepasst. Noch heute unterstützt es Arbeitslose bei der Unternehmensgründung.

\section{Hintergrund}

Ein gängiges Argument zur Rechtfertigung von Gründungszuschüssen für Arbeitslose basiert auf dem Vorhandensein von Unzulänglichkeiten der Kapitalmärkte und einer möglichen Diskriminierung von Arbeitslosen, was zu einer suboptimalen Unternehmensgründungsquote und unterkapitalisierten Unternehmen führen kann (Meager, 1996; Perry, 2006). Gründungszuschüsse zielen durch die Deckung der Lebenshaltungskosten und der Sozialversicherungsbeiträge während des entscheidenden Gründungszeitraums darauf ab, den Schwierigkeiten entgegenzuwirken, die Arbeitslose bei der Finanzierung von Unternehmensgründungen haben. Es kann argumentiert werden, dass Gründungszuschüsse nicht nur zu mehr Unternehmensgründungen anregen, sondern während der Zeit der selbstständigen Erwerbstätigkeit auch die Beschäftigungsfähigkeit, das Humankapital und die Arbeitsmarktnetzwerke derer verbessern, die diese Art der Unterstützung erhalten und ein eigenes Unternehmen gründen. Damit sind sie im Falle eines Scheiterns besser positioniert, eine reguläre Beschäftigung zu finden. Gründungszuschüsse können auch aus makroökonomischer Perspektive vielversprechend sein, da das Entstehen neuer Firmen im Allgemeinen den Wettbewerb, die Innovationskraft und die Produktivität von Firmen erhöht. Investitionen in Start-ups, die von vormals Arbeitslosen geführt werden, könnten insbesondere in Zeiten von Wirtschaftskrisen relevant sein, wenn neue Unternehmensgründungen ein wichtiger Einflussfaktor für wirtschaftliche Trendwenden und neues Wachstum werden könnten. Volkswirtschaften, die mit einer Wirtschaftskrise und hohen Arbeitslosenquoten konfrontiert sind, stehen vor Herausforderungen, 
aber auch vor Chancen für neue Tätigkeiten; während die Marktnachfrage eingeschränkt ist, gibt es ein größeres Angebot an gut ausgebildeten Arbeitskräften, die bereit für eine Unternehmensgründung oder die Aufnahme einer Tätigkeit in neuen Unternehmen sind (Fritsch et al., 2015).

Das direkte Ziel des Programms ist die Deckung der grundlegenden Lebenshaltungskosten und der Sozialversicherungsbeiträge während der Anfangsphase der Unternehmensgründung, wenn das Unternehmen möglicherweise noch nicht genug Erträge abwerfen kann. Das übergeordnete Ziel des Programms besteht darin, die Überlebenswahrscheinlichkeit der Unternehmen zu erhöhen, während sie noch anfällig sind, und den Unternehmern zu ermöglichen, mit ihrer selbstständigen Erwerbstätigkeit angemessene Einnahmen zu generieren, sobald sie die erste Phase der Unternehmensgründung überstanden haben. Mit dieser Maßnahme strebt die Regierung eine Integration dieser Menschen in den Arbeitsmarkt und eine Stärkung ihrer langfristigen Bindung an den Arbeitsmarkt an. Somit wird auch eine Rückkehr in eine abhängige Beschäftigung als Erfolg betrachtet (siehe z. B. Caliendo und Kritikos, 2009a).

Auch wenn die Unterstützung von Start-ups, die von Arbeitslosen gegründet werden, eine weltweit häufig umgesetzte Maßnahme ist, bleibt sie in der Regel eine relativ kleine wirtschaftspolitische Komponente. Diese Art von Intervention wird zuweilen sehr kritisch gesehen, da sie unter Umständen nur geringe wirtschaftliche Auswirkungen erzielen kann (Shane, 2009). Allerdings macht man in Deutschland bereits seit vielen Jahren positive Erfahrungen mit derartigen Initiativen.

\section{Aktivitäten}

Vor 2003 stellte der im Jahr 1986 eingeführte Gründungszuschuss das einzige Programm dar, das Arbeitslose unterstützte, die ein eigenes Unternehmen gründen wollten. Bezieher des Überbrückungsgelds erhielten sechs Monate lang denselben Betrag, den sie in Form der Arbeitslosenunterstützung erhalten hätten (zuzüglich einer Pauschale von 68,5\% zur Deckung der Sozialversicherungsbeiträge). Das Überbrückungsgeld wurde im Januar 2003 durch ein zweites Programm, den Existenzgründungszuschuss (Ich-AG) ergänzt. Hierbei bestand die Unterstützung aus einer Pauschalzahlung von 600 EUR monatlich im ersten, 360 EUR monatlich im zweiten und 240 EUR monatlich im dritten Jahr. Beide Programme wurden von einer nationalen Medienkampagne begleitet.

Der neue Gründungszuschuss (und das frühere Überbrückungsgeld) hat Ähnlichkeit mit Programmen in anderen Ländern, unter anderem in Australien, Neuseeland, Schweden und den Vereinigten Staaten. In den letzten Jahren wurden im Vereinigten Königreich, in Finnland und Frankreich neue Programme eingeführt. Was Deutschland im Vergleich zu ähnlichen Programmen in der Europäischen Union zu einem einzigartigen Fall macht, ist die Anzahl der Menschen, die die zwei Programme in Anspruch nehmen (wobei hier klarzustellen ist, dass man sich für eines der beiden Programme entscheiden musste). Zwischen 2003 und 2006 nahmen etwa eine Million Menschen an diesen Programmen teil. Weiterhin ist zu bemerken, dass man im Jahr 2010150000 Teilnahmen am reformierten Gründungszuschuss verzeichnete. Im Jahr 2012, nach einer weiteren Reform und niedrigerer Arbeitslosigkeit, fiel die Anzahl der Teilnehmer, die aus der Arbeitslosigkeit heraus ein Unternehmen gründeten, auf 20000.

Da die zwei Programme (d. h. der Gründungszuschuss und das Überbrückungsgeld) sich in Bezug auf die finanzielle Unterstützung und die Dauer stark unterscheiden, zogen sie auch unterschiedliche Typen von Menschen an. Die Teilnehmer am Überbrückungsgeld hatten einen höheren Bildungsstand und in der Vergangenheit ein höheres Einkommen (dadurch wurde das Programm attraktiver, wenn die Höhe des Zuschusses von der Höhe der Arbeitslosenunterstützung abhing), während die Teilnehmer am Gründungszuschuss im Durchschnitt weniger gebildet waren und eine relativ schlechte vorherige Arbeitsmarktleistung vorzuweisen hatten. 2006 wurden die beiden Programme zum neuen Gründungszuschuss zusammengelegt. Dieser wird in diesem Abschnitt noch genauer beschrieben.

Von 2002 bis 2011 verfügten diese Programme über einen Jahreshaushalt von 1,5 Milliarden EUR bis 3,5 Milliarden EUR und wurden jedes Jahr von zwischen 100000 und 250000 Teilnehmern in Anspruch genommen. Laut der offiziellen Statistik (Mikrozensus) wurden während dieses Zeitraums jedes Jahr zwischen 300000 und 400000 Unternehmen gegründet. Somit machten Unternehmensgründungen aus der Arbeitslosigkeit heraus einen bedeutenden Anteil der jährlichen Gründungsaktivitäten aus. Auch die Gründungsaktivitäten insgesamt waren überdurchschnittlich. Ferner waren während dieses Zeitraums 
zwischen 2,1 Millionen und 4,6 Millionen Menschen in Deutschland arbeitslos. Das bedeutet, dass zwischen $3 \%$ (in der Hochphase des Programms) und beinahe 10\% aller Arbeitslosen jährlich das Programm nutzten. In der Tat verfügt das Programm gewissermaßen über eine eingebaute Flexibilität, denn die Ausgaben schwanken mit der Arbeitslosigkeit: Die Kosten sind hoch (bzw. niedrig), wenn die Arbeitslosenquoten hoch (bzw. niedrig) sind. Dies lässt sich mit dem Umstand erklären, dass Gründungszuschüsse besonders attraktiv sind, wenn die Arbeitslosigkeit hoch und Beschäftigungsalternativen rar sind (Fritsch et al., 2015).

Das Programm bietet finanzielle Unterstützung für Arbeitslose (und diejenigen, denen Arbeitslosigkeit droht), die ein eigenes Unternehmen gründen möchten. Die öffentlicheArbeitslosenversicherungunterstützt die ersten sechs Monate der selbstständigen Erwerbstätigkeit (und bis zu neun weitere Monate). In den ersten sechs Monaten erhält der Empfänger denselben Betrag, den er an Arbeitslosenleistungen erhalten hätte; hinzu kommt ein monatlicher Betrag über 300 EUR zur Deckung der Sozialversicherungsbeiträge. Die Zahlung der Pauschale von 300 EUR kann um weitere neun Monate verlängert werden, wenn die selbstständige Erwerbstätigkeit die Haupttätigkeit der Person ausmacht und sie Belege für ihre Geschäftstätigkeit vorlegen kann.

Um diesen Zuschuss zu erhalten, muss die Person noch einen Anspruch auf Arbeitslosenunterstützung von mindestens 150 Tagen haben und einen Geschäftsplan vorlegen, der durch die Handelskammer oder eine ähnliche Einrichtung genehmigt wurde. Diese externe Einrichtung muss prüfen, inwiefern die in dem Plan beschriebene Geschäftsidee nachhaltig ist und das Potenzial hat, eine ausreichende Ertragsgrundlage für den Antragsteller zu schaffen. Außerdem werden Unternehmensgründungen nur dann unterstützt, wenn sie als Haupterwerb geplant sind.

Seitdem es den Zuschuss gibt, hatten die Arbeitslosen die meiste Zeit Anspruch auf den Zuschuss, sofern sie die Voraussetzungen erfüllten. Eine Änderung Ende 2011 sorgte dafür, dass der Zuschuss zu einer Ermessensleistung wurde, für den die Bewilligung eines Sachbearbeiters des lokalen Arbeitsamtes (zusätzlich zur externen Genehmigung des Geschäftsplans) erforderlich war. Der Hauptgrund für diese Reform war die Notwendigkeit, die öffentlichen Ausgaben zu senken. Nach der Reform schränkten die lokalen Arbeitsämter die Bewilligungen für den Gründungszuschuss stärker ein, sodass die Anzahl der geförderten Unternehmensgründungen von $120000 \mathrm{im}$ Jahr 2011 auf 20000 im Jahr 2012 und eine ähnlich niedrige Zahl im Jahr 2013 fiel.

\section{Projektfinanzierung}

Die durchschnittlichen Programmkosten pro Person bewegten sich von 2002 bis 2011 zwischen 11000 EUR und 13000 EUR, abhängig von der individuellen Höhe des Anspruchs auf Arbeitslosenleistungen (die wiederum vom vorherigen Arbeitseinkommen abhängen) sowie der Genehmigung der Zuschussverlängerung um neun Monate.

Das Instrument kann als relativ kostengünstig betrachtet werden, denn im Alternativfall wären die meisten der Empfänger für ungefähr denselben Zeitraum, manchmal auch länger, weiterhin arbeitslos und würden dieselbe Geldsumme als Unterstützung beziehen. Es wird aus der gesetzlichen Arbeitslosenversicherung bezahlt.

\section{Herausforderungen}

Bezüglich der finanziellen Förderung von Unternehmensgründungen durch Arbeitslose gibt es einige Bedenken. Zunächst könnten die unterstützten Personen auch ohne diese finanzielle Förderung eine selbstständige Erwerbstätigkeit aufnehmen. Dies wird als Verlust wegen Mitnahmeeffekten bezeichnet und ist in der Regel schwierig festzustellen. Weiterhin bestehen Bedenken wegen Verdrängungseffekten, wenn etablierte oder nicht bezuschusste Firmen unter Umständen durch geförderte Start-ups verdrängt werden. Und schließlich könnten Firmen Mitarbeiter mit bezuschussten Selbstständigen ersetzen (im Sinne einer Scheinselbstständigkeit). Dabei handelt es sich um Arbeitskräfte, die selbstständig erwerbstätig zu sein scheinen, in der Praxis jedoch Angestellte sind. Auf stark regulierten Arbeitsmärkten könnten die Auswirkungen einer solchen Ersetzung eine gewisse Rolle spielen.

Es wird zudem häufig argumentiert, dass Unternehmensgründungen aus der Arbeitslosigkeit heraus weniger produktiv sind und mit geringerer Wahrscheinlichkeit weitere Arbeitsplätze schaffen, da sie als Reaktion auf eine Notwendigkeit und eine sich bietende Möglichkeit erfolgen. Allerdings zeigen 
Evaluierungsstudien, dass in Deutschland rund 85\% der Start-ups entweder ausschließlich aus einer sich bietenden Möglichkeit heraus entstanden sind oder das Ergebnis einer gemischten Motivation aus Möglichkeit und Notwendigkeit waren (Caliendo et al., 2006; Caliendo et al., 2007; Caliendo und Kritikos, 2009a; 2009b; 2010; Caliendo und Künn, 2011; Caliendo et al., 2012).

Weitere Herausforderungen ergeben sich aus der Umsetzung solcher Instrumente. Als größte Herausforderung stellte sich beim deutschen Ansatz die Auswahl qualifizierter externer Einrichtungen heraus, die die Geschäftspläne der Antragsteller angemessen darauf hin überprüfen können, ob sie das Potenzial zur Schaffung einer ausreichenden Ertragsgrundlage für den Antragsteller besitzen. Im deutschen System wurden hierfür Handelskammern, Steuerberater und ähnliche Einrichtungen herangezogen. Sie müssen ihre Entscheidungen dahin gehend austarieren, dass sie einerseits nicht zu streng sind, indem sie einen Vorschlag nicht durchgehen lassen, der erfolgreich hätte sein können, und dass sie andererseits nicht streng genug sind und Vorschläge bewilligen, die wenig Aussichten haben, ein angemessenes Einkommen für den Antragsteller zu generieren.

Ein zweites Problem bei der Umsetzung ist, dass die ausgewählten Einrichtungen kein eigenes Interesse bei der Genehmigung oder Ablehnung von Geschäftsplänen haben sollten. Für Handelskammern könnte zum Beispiel der Anreiz bestehen, die bereits vorhandenen Unternehmen auf dem Markt zu „schützen“ und deshalb zu viel Strenge walten zu lassen. Steuerberater wurden häufig mit dem Vorurteil konfrontiert, nicht streng genug zu sein, da sie sich künftige Klienten sichern könnten, indem sie alle möglichen Geschäftspläne genehmigen. In Retrospektive kann in Bezug auf die deutsche Erfahrung der Schluss gezogen werden, dass die Qualität des Geschäftsplans wahrscheinlich nur eine kleine Rolle spielte. Stattdessen macht es den Anschein, dass der wichtigste Aspekt des Geschäftsplans darin bestand, die Antragsteller dazu zu bringen, sich ausreichend um die Erstellung und Genehmigung eines Geschäftsplans zu bemühen, um die Wahrscheinlichkeit von Verlusten aufgrund von Mitnahmeeffekten zu verringern.

Weiterhin bei der Umsetzung wesentlich ist die Frage, ob ein Anspruch auf den Gründungszuschuss bestehen sollte, sobald alle Voraussetzungen erfüllt sind, oder ob Sachbearbeiter (der Arbeitsagentur) Ermessensentscheidungen treffen sollten. In Deutschland sorgte die jüngste Reform dafür, dass der Anspruch auf den Gründungszuschuss von einer Ermessensentscheidung des Sachbearbeiters des lokalen Arbeitsamts abgelöst wurde. Seither ist die Anzahl der geförderten Unternehmensgründer drastisch gefallen. Allerdings zeigen empirische Belege deutlich, dass es wirksam ist, den Gründungszuschuss als Anspruch (d. h. ohne weitere Überprüfung durch einen lokalen Sachbearbeiter) anzubieten, wenn es das Ziel ist, im großen Rahmen für das Programm zu werben. Andererseits müssen die Zugangsvoraussetzungen so streng festgelegt sein, dass in dem Programm ein wirksames Selbstselektionsverfahren sichergestellt ist.

Und nicht zuletzt sollte betont werden, dass solch ein Instrument nur geringe Auswirkungen auf die Arbeitslosigkeit haben wird, wenn es als isoliertes Programm und ohne die erforderlichen ergänzenden Maßnahmen eingeführt wird. Konkret ist solch ein Programm kein Ersatz für andere Reformen. In Deutschland wurde der Gründungszuschuss zur Erfolgsgeschichte, weil er gemeinsam mit einer großen Arbeitsmarktreform eingeführt wurde.

\section{Auswirkungen}

Der neue Gründungszuschuss und seine Vorgänger waren Gegenstand umfassender Kurzzeit- und Langzeitevaluierungen (Caliendo et al., 2006; Caliendo et al., 2007; Caliendo und Kritikos, 2009a; 2009b; 2010; Caliendo und Künn, 2011; Caliendo et al., 2012). Ein erster Hinweis bei der Messung des Erfolgs von Start-ups ist ihre Überlebensrate. Laut Schätzungen kürzlich durchgeführter Untersuchungen liegen die Überlebensraten der Unternehmen 56 Monate nach der Gründung abhängig von Geschlecht und Region bei $55 \%$ bis 70\%. Frühere Überlebensanalysen (ohne Berücksichtigung des früheren Beschäftigungsstatus) zeigten eine $50 \%$ ige Überlebenschance nach fünf Jahren. Jedoch stellt nicht jede Unternehmensaufgabe automatisch ein Scheitern dar, denn ein sekundäres Ziel des Unterstützungsprogramms besteht darin, Menschen die Rückkehr auf den Arbeitsmarkt zu ermöglichen. Zwischen $20 \%$ und $23 \%$ fanden zu gegebener Zeit eine reguläre Anstellung, sodass nur ein äußerst geringer Bruchteil wieder in die Arbeitslosigkeit bzw. Nichterwerbstätigkeit zurückkehrte.

Bis zu 40\% der Gründer hatten mindestens einen Mitarbeiter. Die Anzahl der von den Gründern beschäftigten Personen schwankte im Durchschnitt zwischen 2,6 und 5,1. Durchschnittlich wurde pro 
Zuschuss ein zusätzlicher Arbeitsplatz geschaffen, d. h.,je 100000 bezuschusste Unternehmensgründungen wurden 100000 zusätzliche Arbeitsplätze geschaffen.

Vergleicht man das Abschneiden der Teilnehmer am Gründungszuschuss mit dem einer aus sonstigen Arbeitslosen bestehenden Kontrollgruppe anhand von statistischen Matching-Verfahren, zeigt das Programm äußert positive Effekte. Die Teilnehmer sind rund 20 Monate länger beschäftigt (in einer selbstständigen oder einer abhängigen Erwerbstätigkeit) und ihr monatliches Arbeitseinkommen liegt 700 EUR (Männer) bzw. 300 EUR (Frauen) höher als in der Vergleichsgruppe.

Die Verluste wegen Mitnahmeeffekten entsprechen dem Anteil der Begünstigten, die auch ohne staatliche Unterstützung ein eigenes Unternehmen gegründet und die ersten Phasen der selbstständigen Erwerbstätigkeit auch ohne diese finanzielle Förderung überlebt hätten. Laut den neuesten Schätzungen betragen die Verluste wegen Mitnahmeeffekten etwa 15\%.

Verdrängungs- und Ersetzungseffekte, die bereits existierenden Unternehmen schaden, scheinen auch begrenzt zu sein. Angesichts der Tatsache, dass das Programm den Begünstigten nur für einen kurzen Zeitraum ein Transfereinkommen garantiert, können sie nur schwerlich anderen Unternehmen schaden, indem sie Produkte zu Dumpingpreisen anbieten.

Die Längsschnittanalyse zeigt, dass die Mehrheit der Unternehmensgründer im ersten Jahr nach der Gründung niedrigere Einnahmen generieren als zuvor, als sie noch beschäftigt waren (dies entspricht den Erwartungen und stützt das Argument für das vorliegende Programm). In den darauffolgenden Jahren ihrer Selbstständigkeit erwirtschaftete mehr als die Hälfte der Unternehmer ein höheres Einkommen durch die selbstständige Erwerbstätigkeit als in ihrer vorherigen Position als Angestellte.

\section{Voraussetzungen für die Übertragung}

Die Fülle an Belegen aus Evaluierungen deutet auf eine Reihe wichtiger Erfolgsfaktoren für das Programm hin, aus denen Erkenntnisse für die Entwicklung von Gründungszuschüssen in anderen Ländern gezogen werden können:

1. Unterstützung des Lebensunterhalts. Der Zeithorizont für die finanzielle Komponente sollte weder zu kurz noch zu lang sein. Die deutsche Erfahrung sieht eine geplante Dauer von sechs bis 15 Monaten vor, wobei der finanzielle Transfer den andernfalls bezogenen Arbeitslosenleistungen entspricht.

2. Einsatz eines Screening-Mechanismus. Die Auflage, einen Geschäftsplan zu entwickeln und ihn durch Dritte genehmigen zu lassen, trägt dazu bei, sicherzustellen, dass nur diejenigen Zugang zu dem Programm erhalten, die ernsthaft eine Unternehmensgründung beabsichtigen. Darum muss gewährleistet sein, dass diese Dritten bei der Genehmigung von Geschäftsplänen objektive Gutachter sind. Der wichtigste Aspekt besteht darin, dass die Zugangsvoraussetzungen über den Geschäftsplan oder andere Elemente in dem Programm zu einer wirksamen Selbstselektion führen müssen. Bei der Vorbereitung und Genehmigung eines Geschäftsplans müssen ausreichend große Bemühungen stattfinden, um eine Selbstselektion sicherzustellen und um Verluste wegen Mitnahmeeffekten auszuschließen.

3. Einsatz zeigen. Eine weitere wichtige Voraussetzung des Programms ist, dass die Teilnehmer für den Zugang zum Programm auf ihre Arbeitslosenleistungen verzichten müssen. Laut der deutschen Erfahrung ist eine Quote von 1:1 angemessen. Dies zeigt den Einsatz der Teilnehmer und stellt sicher, dass sie nicht nur die Bezugsdauer ihrer Leistungen maximieren wollen.

4. Durchführung von Kontaktaufnahmen. Eine entscheidende Frage betrifft die Kontaktarbeit des Programms. Gründungszuschüsse werden nur in Deutschland von einer erheblichen Anzahl von Menschen in Anspruch genommen, während die Teilnehmerzahl in anderen Ländern etwa $10 \%$ oder weniger im Vergleich zu der in Deutschland beträgt. Außerdem scheinen für öffentliche Beihilfen besondere Werbemaßnahmen notwendig zu sein. In Deutschland wurde für die Förderung von Existenzgründungen aus der Arbeitslosigkeit heraus in großem Umfang in Zeitungen und anderen Massenmedien geworben. Darüber hinaus machten Arbeitslosenagenturen regelmäßig Ankündigungen über diese Programme und sorgten damit für einen hohen Bekanntheitsgrad dieser Förderung.

5. Nicht jeder ist ein Unternehmer. Es sollte klar sein, dass nicht jeder Arbeitslose geneigt ist, selbstständig erwerbstätig oder als Unternehmer tätig zu sein. Darum muss betont werden, dass für ein solches Programm restriktive Zugangsvoraussetzungen gelten müssen, damit es wirksam und nutzbringend 
ist. Um das Wachstum der neu gegründeten Unternehmen anzukurbeln und die Unternehmer zu fördern, die den Aufbau eines größeren Unternehmens anstreben, könnte solch ein Programm von einer anfänglichen und einer laufenden Unterstützung (zum Beispiel durch Coaching-Angebote) gerahmt werden.

\section{Literatur}

Caliendo, M., J. Hogenacker, S. Künn und F. Wießner (2012), „Alte Idee, neues Programm: Der Gründungszuschuss als Nachfolger von Überbrückungsgeld und Ich-AG“, Journal for Labour Market Research, Bd. 45, S. 99-123.

Caliendo, M. und A. Kritikos (2010), „Start-ups by the Unemployed: Characteristics, Survival and Direct Employment Effects“, Small Business Economics, Bd. 35, S. 71-92.

Caliendo, M. und A. Kritikos (2009a), „Die reformierte Gründungsförderung für Arbeitslose - Chancen und Risiken“, Perspektiven der Wirtschaftspolitik, Bd. 10, S. 189-213.

Caliendo, M. und A. Kritikos (2009b), „IWant to, But I also Need to: Start-Ups Resulting from Opportunity and Necessity“, Diskussionspapier Nr. 4661, IZA Bonn.

Caliendo, M., A. Kritikos, V. Steiner und F. Wießner (2007), „Existenzgründungen: Unterm Strich ein Erfolg“, IAB-Kurzbericht 10/2007.

Caliendo, M., A. Kritikos und F. Wießner (2006), „Existenzgründungsförderung in Deutschland, Zwischenergebnisse aus der Hartz-Evaluation“, Journal for Labour Market Research, Bd. 39, S. 505-531.

Caliendo, M. und S. Kuenn (2011), „Start-up subsidies for the unemployed: Long-term evidence and effect heterogeneity“, Journal of Public Economics, Bd. 95, S. 311-331.

Fritsch, M., A. Kritikos und K. Pijnenburg (2015), „Business Cycles, Unemployment and Entrepreneurial Entry“, International Entrepreneurship and Management Journal, Bd. 11, S. 267-286.

Meager, N. (1996), „From Unemployment to Self-Employment: Labour Market Policies for Business Start-up“, in International Handbook of Labour Market Policy and Evaluation, Hrsg. G. Schmidt, J. O’Reilly und K. Schomann, S. 489-519, Edward Elgar.

Perry, G. (2006), „Are Business Start-up Subsidies Effective for the Unemployed: Evaluation of Enterprise Allowance“, Working Paper, AUT: Auckland.

Shane, S. (2009), „Why encouraging more people to become entrepreneurs is bad public policy“, Small Business Economics, Bd. 33, S. 141-149. 


\section{Kiútprogram, Ungarn}

Diese Fallstudie stellt ein integriertes Projekt vor, das Roma bei der Unternehmensgründung unterstützte. Die Fallstudie erörtert die Ziele dieses Ansatzes, den Hintergrund sowie die Entwicklung der Aktivitäten und die Gründe für Änderungen des Ansatzes. Auch die Auswirkungen des Projekts werden vorgestellt. Es folgt eine Erörterung der Herausforderungen im Zusammenhang mit der Umsetzung und der Voraussetzungen für eine Übertragung.

\section{Ziele}

Kiútprogram lief von 2010 bis 2012. Das Hauptziel des Programms war die Förderung der sozialen Mobilität und der Eingliederung benachteiligter Menschen in benachteiligten Gebieten, indem diesen durch die Gründung und Eintragung eines Kleinunternehmens die Aufnahme einer selbstständigen Erwerbstätigkeit ermöglicht wurde. Außerdem Zugang zu dem Projekt hatten Menschen, die ihre informelle Geschäftstätigkeit in ein legales Unternehmen umzuwandeln wünschten. Zur Unterstützung von Unternehmensgründungen bot das Programm Mikrokredite und ergänzende Unternehmensförderungsdienste (z. B. Mentoring und Schulungen) an. Die Zielgruppe bestand aus in Armut lebenden Menschen. Das Programm richtete sich ausdrücklich, aber nicht ausschließlich, an Roma-Frauen und -Männer.

Die Gründer und Sponsoren des Programms definierten die Initiative als „soziales Mikrokreditprogramm“, was auf einen ausgewogenen Ansatz zwischen sozialer Kohäsion und finanziellen Zielen hindeutet. Mit dem Programm versuchte man, die sozialen Einstellungen gegenüber benachteiligten Gruppen wie den Roma zu verbessern und die Diskriminierung dieser Gruppen zu mindern.

\section{Hintergrund}

In Ungarn liegt der Anteil der selbstständig Erwerbstätigen Roma an der erwerbstätigen Bevölkerung insgesamt erheblich unter dem der allgemeinen Bevölkerung (5\% gegenüber $15 \%$ ). Außerdem haben von allen Roma in der gesamten Region Zentral- und Osteuropa die Roma in Ungarn die niedrigste Selbstständigenquote. Andererseits würden $24 \%$ der ungarischen Roma lieber ein Unternehmen gründen, als in einem Angestelltenverhältnis zu arbeiten (Weltbank - UNDP, 2012).

In den meisten Ländern mit einer bedeutenden Roma-Minderheit stehen die Menschen dieser Gruppe beim (Wieder-)Eintritt in den Arbeitsmarkt als selbstständig Erwerbstätige vor enormen Herausforderungen. Untersuchungen belegen, dass die größten Hindernisse bei der Unternehmensgründung ein beschränkter Zugang zu Finanzdienstleistungen (einschließlich des Zugangs zu Bankkonten und zu persönlichen Krediten und Darlehen) und begrenzte Erfahrungen mit der Führung eines Unternehmens sind. Außerdem haben die Roma in der Regel einen niedrigen Bildungsstand und verfügen über ein sehr geringes Finanzwissen (Molnár, 2012; Weltbank - UNDP, 2012). Die Situation der Roma in Ungarn wird zusätzlich erschwert durch starke negative Stereotype, die in der Gesellschaft und den Medien zum Ausdruck kommen (Bernáth-Messing, 2013). Die Vorurteile halten sich hartnäckig, und die Durchsetzung von Antidiskriminierungsgesetzen und Verwaltungsverfahren zur Bekämpfung ethnischer Diskriminierung ist schwach (Majtényi, 2009).

Das Ziel von Kiútprogram war es, sowohl Markt- als auch Staatsversagen anzugehen. Es konzentrierte sich auf abgelegene, kleine Regionen Ungarns, wo die Wirtschaftstätigkeit gering und die öffentliche Infrastruktur unterentwickelt ist und Unternehmensinvestitionen weit unter dem nationalen Durchschnitt liegen. Die Beschäftigungsmöglichkeiten in diesen Regionen beschränken sich 
in der Regel auf die Beteiligung an öffentlichen Arbeitsprogrammen, die nur befristete Arbeitsplätze bieten (BI, 2011), oder an der informellen Wirtschaft. Dieses Programm wurde ins Leben gerufen, um Roma bei der Deckung der Kosten für die Unternehmensgründung zu unterstützen und sie dazu zu ermutigen, als formelles und nicht als informelles Unternehmen zu agieren (Reszkető-Váradi, 2012; Molnár, 2012).

\section{Aktivitäten}

Die Polgár Stiftung für Möglichkeiten, eine ungarische Bürgerstiftung, initiierte das Projekt im Jahr 2008 mit der Idee, das Grameen-Modell ( $\left.{ }^{1}\right)$ an den ungarischen Kontext anzupassen. Sie gab eine Machbarkeitsstudie in Auftrag, die aus Feldforschung bestand, um die Notwendigkeit eines solchen Programms zu analysieren. Im Anschluss an die Machbarkeitsstudie reichte die Stiftung im September 2009 im Rahmen eines Ausschreibungsverfahrens der Europäischen Kommission ein Angebot für ein Pilotprojekt im Bereich Mikrofinanzierung ein. Dieser Antrag basierte auf den Ergebnissen der vorbereitenden Forschungsstudie, die die potenziellen Zielregionen und die Bedürfnisse der lokalen Roma-Gemeinschaften erfasste (Molnár, 2012; Szuhai, 2008). Im März 2010 leistete die Europäische Kommission Unterstützung beim Start des Programms als europäisches Pilotprojekt für Mikrofinanzierung im Zeitraum von Oktober 2010 bis Juni 2012. Die Polgár-Stiftung war einer der Schirmherren.

Gemäß ungarischem Finanzrecht darf die Auszahlung von Kreditprodukten nur über Finanzinstitute erfolgen. In der Folge wurde die Raiffeisen Bank Finanzverwaltungspartner des Projekts. Die Bank zahlte die Darlehen aus und verwaltete die Ausgaben und Verluste im Rahmen ihres Unternehmensprogramms für soziale Verantwortung. Die Bank bot den Klienten zudem Sparkonten an und schulte die Bankmitarbeiter in regional ausgewählten lokalen Filialen, um sie bei der Arbeit mit dieser Klientenbasis zu unterstützen.

Die Kiútprogram Microcredit Initiative Non-profit Ltd. (die Verwaltungsgesellschaft) wurde im September 2009 gegründet. Die Verwaltungsgesellschaft war für die Durchführung des Programms verantwortlich, unter anderem für die Schulung und Koordination der Außendienstmitarbeiter, die Überprüfung und Schulung von Klienten, die Bereitstellung zusätzlicher Wirtschaftsförderungsdienste und die Verwaltung der Darlehensverträge in Zusammenarbeit mit der Raiffeisen Bank. Die Weltbank und das Entwicklungsprogramm der Vereinten Nationen (United Nations Development Programme, UNDP) unterstützten das Projekt mit technischem Fachwissen bei der Überwachung, d. h. der Gestaltung der Überwachungsdatenbank und der Erhebung und Analyse der Überwachungsdaten.

Die Außendienstmitarbeiter waren die zentralen Akteure bei der Bereitstellung der Dienste des Programms. Sie waren zuständig für die Erfassung und Überprüfung der potenziellen Siedlungen und Teilnehmer, die Bereitstellung der Schulungen und individueller Unternehmensförderung auf lokaler Ebene, die Förderung und Unterstützung der Erstellung individueller Geschäftspläne und die Erleichterung der Durchführung der Darlehensgruppen. Mit fortschreitender Umsetzung waren sie zudem die Schlüsselmitarbeiter des Programms, die für die Überwachung der Tätigkeiten der Kleinunternehmen, die Organisation der nach der Auszahlung stattfindenden Sitzungen der Darlehensgruppen und für die frühzeitige Meldung potenziell ausbleibender Rückzahlungen oder sonstiger Notfälle an die Bank zuständig waren. Die meistenAußendienstmitarbeiter waren ausgebildete Sozialarbeiter und wurden aufgrund ihrer Arbeitserfahrung ausgewählt. Alle Außendienstmitarbeiter durchliefen vor ihrem Einsatz im Außendienst ein einmonatiges Schulungsmodul.

Die Klienten wurden aus Regionen mit weniger als $60 \%$ des Medianeinkommens der ungarischen Haushalte ausgewählt. Das Programm räumte Frauen Priorität ein, jedoch waren nur $40 \%$ der Bewerber und $48 \%$ der Klienten weiblich. Gespräche mit Klienten - allerdings nicht auf der Basis einer

1 Das Grameen-Modell wurde von Prof. Mohammed Yunus in Bangladesch entwickelt. Es folgt einer Methodik, bei der eine Mikrofinanzierungsinstitution in einem Dorf eingerichtet wird, das 15 bis 20 andere Dörfer versorgt. Die Mikrofinanzierungsinstitution gewährt selbst gebildeten Gruppen von jeweils etwa fünf Kreditnehmern Darlehen, für die diese gemeinsam Verantwortung tragen (wie im solidarischen Gruppenmodell). Die Darlehen werden in Wellen gewährt: Die ersten Mitglieder erhalten zunächst ihr Darlehen, dann die nächsten - sofern die ersten Mitglieder ihre Schulden zurückgezahlt haben - und so weiter. Ein Mechanismus, um die Rückzahlung zu verbessern, ist der Gruppendruck (OECD, 2015). 
repräsentativen Stichprobe - deuten darauf hin, dass die offiziellen Zahlen die Teilnahme seitens der Frauen eventuell nach oben verzerren, da in vielen Fällen der Ehemann oder Partner der eigentliche Geschäftsführer war (Weltbank - UNDP, 2012).

Das Auswahlverfahren bestand aus zwei Schritten. Zunächst überprüften die Außendienstmitarbeiter die infrage kommenden Siedlungen. Im Zeitraum zwischen 2010 und 2012 besuchten die Außendienstmitarbeiter 202 Siedlungen, um das Programm öffentlich anzukündigen und Treffen für die lokale Gemeinschaft zu organisieren, auf denen Informationen zu dem Mikrofinanzierungsprogramm und den ergänzenden Unternehmensdienstleistungen gegeben wurden. Als die Gemeinschaften großes Interesse an dem Programm zeigten, besuchten Außendienstmitarbeiter Familien, um die wirtschaftlichen und sozialen Bedingungen der potenziellen Teilnehmer vor Ort beurteilen zu können. Während dieser Besuche füllten sie außerdem den Aufnahmefragebogen zusammen mit den Familien aus, um grundlegende Informationen über die Bewerber und ihre Haushalte zu sammeln.

In dieser Phase des Auswahlverfahrens wurden Darlehensgruppen mit je acht oder neun Kandidaten gebildet. Gruppentreffen wurden ausgerichtet, und die Außendienstmitarbeiter sondierten, welche frühere Geschäftserfahrung die Gruppenmitglieder hatten. Die Gruppe musste einen Leiter wählen und die Gruppenregeln billigen. Potenzielle Kandidaten mussten außerdem eine Reihe von Auswahlkriterien erfüllen (z. B. durften sie keine Steuerschulden haben) und einen einfachen, aber realistischen Entwurf für einen Geschäftsplan erstellen. Der Kreditausschuss der Verwaltungsgesellschaft bewertete die individuellen Bewerbungspakete, die den vor Ort ausgefüllten Fragebogen, eine Evaluierung durch den Außendienstmitarbeiter, den Geschäftsplanentwurf und eine individuelle Selbstbewertung umfassten, und trafen in Abstimmung mit dem Außendienstmitarbeiter die endgültige Entscheidung.

In Anlehnung an das Grameen-Modell basierte dieses Programm auf der Gruppenkreditvergabe. Die Darlehensgruppen dienten als Ersatz für die fehlenden Sicherheiten und als Forum für Erfahrungsaustausch und gegenseitiges Lernen. Die Gruppendynamik und die Betriebsregeln sollten die Rückzahlung verstärken, da die Gruppenmitglieder ihre Darlehen der Reihe nach und unter der Voraussetzung erhielten, dass es keine Zahlungsausfälle gab. Der Gruppenleiter erhielt seinen Kredit als Letzter. Die Darlehen wurden gemäß drei verschiedenen Plänen zur Verfügung gestellt: Darlehen in Höhe von 670 EUR waren über sechs Monate, Darlehen in Höhe von 1670 EUR über zwölf Monate und Darlehen in Höhe von 3330 EUR über 18 Monate zurückzuzahlen.

\section{Entwicklung des Projekts}

Anfang 2011 wurde klar, dass eine Anpassung der Projektkonzeption vonnöten war. Im Herbst 2010 erfolgte die Rekrutierung von Klienten viel langsamer als erwartet. Bis Ende 2010 hatte das Programm elf Darlehensgruppen mit rund 70 Teilnehmern. Dies lag unter dem anfänglichen Programmziel von 100 Klienten bis Ende des ersten Jahres. Damit stand auch das Gesamtziel von 400 Klienten auf dem Spiel. Außerdem war die Ausfallquote sehr hoch: Bei $80 \%$ der Darlehen gab es einen Zahlungsverzug oder -ausfall.

Während der ersten Hälfte des zweiten Jahres wurden die folgenden Änderungen vorgenommen:

- Verstärkte Überprüfung von Klienten und Verlagerung der Zielregionen. Die Außendienstmitarbeiter konzentrierten sich verstärkt auf die Erfassung der Bonitätsgeschichte, der Geschäftsverbindungen und der Erfahrung der potenziellen Klienten und ihrer Familienmitglieder. Sie zogen sich außerdem aus den am stärksten benachteiligten kleinen Regionen zurück. Im Ergebnis hatten potenzielle Klienten, die bereits Geschäftserfahrungen oder zumindest Verbindungen zu bestehenden Unternehmernetzwerken hatten, bessere Chancen, Kandidaten zu werden. Dies führte auch dazu, dass die neue Zielgruppe aus einer etwas bessergestellten wirtschaftlichen Schicht kam.

- Erweiterung des Umfangs der unterstützten Geschäftstätigkeiten. Aufgrund einer entsprechenden Nachfrage seitens der Klienten wurde die Landwirtschaft als ein möglicher Sektor für die Unternehmensgründung ergänzt. 
- Durchsetzung der Rückzahlungen und Gruppendynamik. Das Programm gestattete die Bildung von Darlehensgruppen mit weniger als fünf Mitgliedern - eine Mindestgrenze, die während des ersten Zeitraums strikt durchgesetzt wurde. Die Darlehensgruppen setzten zudem verstärkt ihren Schwerpunkt auf die Entwicklung von Geschäftsplänen und führten strengere Evaluierungskriterien ein.Außerdem wurde die durchschnittliche Höhe der Darlehen auf1832EUR und die durchschnittliche Laufzeit auf 43 Wochen herabgesetzt und für mehr Flexibilität bei der Umschuldung von Darlehen gesorgt. Dies trug dazu bei, sicherzustellen, dass die Klienten auch nach Ende der ersten sechs Monate ihrer Geschäftstätigkeit zahlen konnten. Zusätzlich erhielten die Darlehensgruppen mehr Unterstützung beim Zugang zum staatlichen Förderprogramm Vállalkozóvá Válási Támogatás, das Start-ups während der ersten sechs Monate ihrer Geschäftstätigkeit zur Verfügung stand. Eine Teilnahme an diesem Programm ermöglichte die Refinanzierung der Steuerzahlungen und Sozialversicherungsbeiträge. (Laut den nationalen Vorschriften waren selbstständig Erwerbstätige verpflichtet, Steuern und Sozialversicherungsbeiträge auf der Grundlage des für das jeweilige Steuerjahr festgelegten Mindestlohns zu zahlen, unabhängig von ihren tatsächlichen Einnahmen).

- Verstärkung der Verwaltung und Verbesserung der Schulung von Außendienstmitarbeitern. Das Programm richtete einen Krisenausschuss ein, der Klienten, die bei ihren Zahlungen in Verzug zu kommen drohten, individuell unterstützten, und stellte neue Außendienstmitarbeiter mit mehr Erfahrungen in der Sozialarbeit ein. Auf die Rückmeldung der Klienten hin wurden die Außendienstmitarbeiter außerdem zusätzlich geschult. Ferner ernannte das Programm regionale Koordinatoren, um die Kommunikation unter den Außendienstmitarbeitern und zwischen ihnen und dem Projektbüro zu verbessern.

\section{Projektfinanzierung}

Das Projekt „Pan-European Coordination of Roma Integration Methods - Roma Inclusion: Selfemployment and microcredit" (Paneuropäische Koordination von Integrationsmethoden für Roma Einbeziehung der Roma: Selbstständige Erwerbstätigkeit und Mikrokredite) der Europäischen Union stellte die Hauptfinanzierungsquelle für das Kiútprogram dar. Der Gesamthaushalt des Projekts einschließlich Betriebskosten und Darlehensbestand - belief sich auf 1,4 Millionen EUR. Das Projekt der Europäischen Union stellte eine Vorfinanzierung (85\%) bereit. Der übrige Anteil wurde nach dem offiziellen Abschluss des Projekts gesichert. Die ungarische Regierung kofinanzierte die Projektmanagementkosten (200 Millionen HUF bzw. etwa 666670 EUR), und andere staatliche Förderungen (Refinanzierung von Steuerzahlungen und Sozialversicherungsbeiträgen) betrugen während des Projektzeitraums 135 Millionen HUF (etwa 450000 EUR). Nach Abschluss der Pilotphase wurden die Folgeaktivitäten des Projekts und einige der lokalen Projekte aus privaten Quellen, hauptsächlich von der Polgár-Stiftung, finanziert.

\section{Herausforderungen}

Das Programm war mit drei großen Herausforderungen konfrontiert. Die erste bestand darin, geeignete Klienten anzusprechen und diejenigen von ihnen zu identifizieren, die das Potenzial hatten, eine selbstständige Erwerbstätigkeit zu meistern. Die Nachhaltigkeit jeglicher Geschäftstätigkeiten in den am stärksten benachteiligten Regionen wurde durch eine eingeschränkte Nachfrage auf den regionalen Märkten und eingeschränkte Unternehmernetzwerke vor eine Herausforderung gestellt. Folglich verlagerte das Kiútprogram seinen Fokus auf "gehobene“ Klienten und auf Gebiete mit besserem Zugang zu regionalen Märkten und bestehenden Unternehmernetzwerken. Dies allerdings untergrub die sozialen Ziele des Programms.

Die zweite Herausforderung war, leistungsfähige Außendienstmitarbeiter zu rekrutieren, die gleichzeitig als persönlicher Mentor, Sozialarbeiter und Kreditsachbearbeiter mit entsprechendem Finanz- und Unternehmensfachwissen auftreten konnte. Verschiedene Menschen für die verschiedenen Aufgaben einzustellen wäre eine Möglichkeit gewesen, die jedoch durch den Projekthaushalt nicht gegeben war. Während des ersten operativen Jahres war die Fluktuation bei den Außendienstmitarbeitern äußerst hoch (rund 90\% der in den ersten Monaten der Projektdurchführung 
eingestellten Außendienstmitarbeiter verließen das Projekt im folgenden Jahr), was darauf hindeutet, wie schwierig diese Aufgabe ist. Die Verwaltungsgesellschaft startete während des zweijährigen Projektzeitraums fünf Aufrufe, um Außendienstmitarbeiter anzuwerben.

Drittens benötigen neue Unternehmen Zeit, um sich zu entwickeln und eine nachhaltige Klientenbasis zu gewinnen. Dies dauert häufig länger als ein Jahr. Das Kiútprogram konnte eine Zusammenarbeit mit den ungarischen öffentlichen Arbeitsverwaltungen aufbauen, um den Zugang neuer Unternehmer zu einem bestimmten staatlichen Beihilfeprogramm während der ersten sechs Monate ihres Betriebs zu garantieren, doch gelang es nicht, einen Partnerschaftsvertrag auf institutioneller Ebene zu schließen, der länger als sechs Monate dauern konnte. Dies stellte eine Herausforderung für die Teilnehmer dar, die einen wöchentlichen Rückzahlungsplan einhalten mussten.

\section{Auswirkungen}

Es gibt keine Ex-post-Evaluierung dieses Projekts. Allerdings wurde in Zusammenarbeit mit der Weltbank und dem Entwicklungsprogramm der Vereinten Nationen (United Nations Development Programme, UNDP) ein umfassendes Überwachungssystem eingerichtet. Überwachungsdaten zeigen, dass sich 138 der insgesamt 192 Teilnehmer einer Darlehensgruppe anschlossen. Davon erhielten 49 Menschen unter diesen Voraussetzungen Darlehen in einer Höhe von durchschnittlich 1825 EUR pro Person und einer durchschnittlichen Laufzeit von 52 Wochen (Reszket冈 und Váradi, 2012; World Bank, 2013).

Dem Projekt gelang es, Arbeitslose (86\% der Bewerber waren vor der Teilnahme mehr als ein Jahr lang arbeitslos oder als nicht erwerbstätig gemeldet) und Haushalte mit sehr niedrigem Monatseinkommen (das Medianeinkommen der Haushalte betrug 68250 HUF bzw. etwa 227 EUR) zu erreichen. Der Anteil der Bewerber mit abgeschlossener Sekundarausbildung oder einem höheren Bildungsgrad war sehr gering (13\%), und $87 \%$ von ihnen lebten im ländlichen Raum.

Die Unternehmen, die eine Finanzierung erhielten, waren entweder in der Land- und Forstwirtschaft (45\%) oder im Einzelhandel (55\%) angesiedelt. Laut der von der Weltbank durchgeführten Teilnehmerbefragung mangelte es $68 \%$ der Klienten an den nötigen finanziellen Mitteln für eine Unternehmensgründung, und $95 \%$ von ihnen gaben an, das Darlehen, das sie durch das Projekt erhalten haben, sei die Hauptquelle ihres Startkapitals gewesen. Diese Unternehmen erwirtschafteten durchschnittlich einen Monatsumsatz von 68312 HUF (etwa 227 EUR). Im Mai 2013, etwa ein Jahr nach dem offiziellen Projektabschluss, waren $45 \%$ der Unternehmen immer noch in Betrieb.

Die Ex-post-Überwachung deutet darauf hin, dass Frauen und Menschen, die sich vor dem Projekt aktiv in die Gemeinschaft einbrachten (d. h., mindestens einmal wöchentlich an irgendeiner Art von Gesprächen und Entscheidungen der lokalen Gemeinschaft mitwirkten), die Rückzahlungen am häufigsten gemäß dem vereinbarten Rückzahlungsplan leisteten (Weltbank - UNDP, 2012). Diese Ergebnisse stimmen mit internationalen Erfahrungen überein und unterstreichen die Bedeutung lokaler Netzwerke und persönlicher Beziehungen.

Ferner betonten Überwachungsstudien die Wichtigkeit eines umfassenden Ansatzes bei der Projektkonzeption; dieser wird aufgrund der Kennzeichnung des Projektes als soziales Mikrokreditprogramm vernachlässigt (Weltbank - UNDP, 2012; Reszket】-Váradi, 2012). Laut beiden Studien wäre eine längere Pilotphase (d. h. mindestens drei oder vier Jahre) von Vorteil gewesen. Außerdem hätten weniger ehrgeizige Ergebnisindikatoren zur Entwicklung hochwertigerer Dienstleistungen zur Unternehmensförderung geführt. Die Studien merken zudem an, dass man die Ausstiegsstrategien der Teilnehmer und ein klares allmähliches Auslaufen der Unterstützung in stärkerem Maße hätte berücksichtigen sollen.

In Bezug auf die Kostenwirksamkeit vergleicht die Weltbank - UNDP (2012) das Kiútprogram mit öffentlichen Arbeitsprogrammen. Sie schätzten die monatlichen Kosten ähnlich hoch ein (2010 EUR pro Monat und Klient), doch wurde angemerkt, dass es dem Kiútprogram besser gelingen könnte, die Aussichten der Klienten auf eine abhängige oder selbstständige Erwerbstätigkeit zu erhöhen. 


\section{Voraussetzungen für die Übertragung}

Dieses Projekt bietet ein Beispiel dafür, wie die am stärksten benachteiligten Mitglieder der Gesellschaft durch das Unternehmertum unterstützt werden können. Die Erfahrung enthält viele Erkenntnisse für ähnliche Initiativen:

1. Sicherstellung leistungsfähiger Außendienstmitarbeiter. Die Außendienstmitarbeiter erwiesen sich als die Schlüsselakteure in der Umsetzungsphase. Aus dem Kiútprogram lassen sich hilfreiche Erkenntnisse für die Auswahl der Außendienstmitarbeiter und die Diversifizierung ihrer Aufgaben ziehen. Die gleichzeitig übernommene Rolle eines Sozialarbeiters und eines Kreditsachbearbeiters und die Komplexität der damit zusammenhängenden Dienstleistungen könnten sich als Hindernis herausstellen. Daher ist es unter Umständen erforderlich, die Aufgaben aufzuteilen und Mitarbeiter einzustellen, die nur auf jeweils eine Rolle spezialisiert sind.

2. Nutzung einer intensiven Kontaktarbeit. Die Absicht des Programms ist es, benachteiligten Gruppen in benachteiligten Gebieten zu helfen. Der Schlüssel, um diese Gruppe zu erreichen und sie auf die verfügbare Unterstützung aufmerksam zu machen, liegt darin, sie vor Ort aufzusuchen und direkt mit ihnen zu interagieren. Dies hat außerdem den Vorteil, dass die Außendienstmitarbeiter dabei potenzielle Kandidaten identifizieren und ein Vertrauensverhältnis zu den Klienten aufbauen können.

3. Realistische Zielsetzung. Dieses Programm musste während der Pilotphase stark angepasst werden, als klar wurde, dass es zu ehrgeizig und zu selektiv war. Im Ergebnis änderte sich das Profil der Zielklienten, und viele der sozialen Ziele wurden gefährdet. Es ist wichtig, eine Ex-ante-Evaluierung durchzuführen, um sicherzustellen, dass die Projektkonzeption den Zielen entspricht.

4. Sicherstellung geeigneter Darlehen. Die Höhe des angebotenen Darlehens sollte auf das Geschäftsumfeld und die Bedürfnisse der Unternehmen der Teilnehmer zugeschnitten sein. Dies bedeutet ein höheres Startkapital in europäischen Ländern als im Kontext der Entwicklungsländer, wo diese Art von Ansatz ursprünglich entstand.

\section{Literatur}

Budapest Institute (2011), „The efficiency of municipal public work programmes“, Budapest Institute. Budapest, verfügbar unter: www.budapestinstitute.eu/uploads/BI_efficiency_publicworks_summary1. $p d f$

Majtényi, B. (Hrsg.) (2009), Lejtős pálya - Antidiszkrimináció és esélyegyenlőség, Földrész Könyvek, L'Harmattan Kiadó.

Molnár, G. (2012), „Kiútprogram - Way out from poverty: Lessons of an Employment-Generating, Social Microlending Programme“, Bratislava.

OECD (2015), „Innovation Policies for Inclusive Development: Scaling up inclusive innovations“, verfügbar unter: www.oecd.org/innovation/inno/scaling-up-inclusive-innovations.pdf.

Reszkető, P. und B.Váradi (2012), „Evaluation of the Hungarian Microfinance Programme - Kiútprogram“, Budapest Institute.

Weltbank (2013), „Kiútprogram Social Microcredit Programme, Case Study“, Weltbank.

Weltbank - UNDP (2012), „Reducing Vulnerability and Promoting the Self-Employment of Roma in Eastern Europe through Financial Inclusion“, Weltbank, verfügbar unter: http://documents. worldbank.org/curated/en/2012/09/16706486/reducing-vulnerability-promoting-self-employment-romaeastern-europe-through-financial-inclusion. 


\section{Going for Growth, Irland}

Diese Fallstudie stellt ein Projekt vor, das Rollenvorbilder einsetzt, um Unternehmerinnen bei der Festlegung und Erreichung von Wachstumszielen für ihre Unternehmen zu animieren und zu unterstützen. Die Fallstudie stellt die Ziele dieses Ansatzes und dessen Hintergrund vor und beschreibt die Aktivitäten und Auswirkungen des Projekts. Außerdem werden die Herausforderungen und die Voraussetzungen für die Übertragung dieses Ansatzes auf einen anderen Kontext erörtert.

\section{Ziele}

Going for Growth konzentriert sich auf die Ermutigung von Unternehmerinnen, in Bezug auf ihr Unternehmen Ehrgeiz zu entwickeln, und unterstützt sie dann bei der Umsetzung ihrer Wachstumsbestrebungen. Das Projekt möchte der Tatsache abhelfen, dass mehr Männer als Frauen wesentliche Wachstumsbestrebungen für ihre Unternehmen haben. Dies hat wirtschaftliche und soziale Folgen sowie Folgen für die Gleichstellung, da die unternehmerischen Bemühungen vieler Unternehmerinnen geringere Erträge generieren als die ihrer männlichen Pendants.

\section{Gründe}

Eine gezielte Maßnahme wurde als notwendig betrachtet, da Frauen Forschungsstudien zufolge nicht nur viel seltener neue Unternehmen gründen als Männer, sondern ihre Unternehmen auch seltener wachsen lassen und stattdessen selbstständig erwerbstätige Einzelunternehmerinnen oder Kleinstunternehmerinnen bleiben (GEM, 2013). Frauen sind im Unternehmertum in der Tendenz weniger selbstbewusst, nehmen unternehmerische Möglichkeiten seltener war und haben weniger entwickelte Netzwerke als Männer. Entsprechend hegt ein kleinerer Anteil der Unternehmerinnen erhebliche Wachstumsbestrebungen (GEM, 2013).

Nachdem man feststellte, dass die Auswirkungen der kleinen Anzahl von Unternehmen, die ein bedeutendes Wachstum erreichten, äußerst wesentlich fürWirtschaft und Beschäftigung sind, erkannte die Europäische Kommission in einem Grünbuch aus dem Jahr 2003 an, dass eine Unterstützung bestehender Unternehmen wichtig sei, damit sie ihr Wachstumspotenzial realisieren können (EK, 2003). Als Antwort auf diese Herausforderung wurde im Jahr 2008 Going for Growth entwickelt. Das Projekt folgte einem Aufruf zur Einreichung von Vorschlägen der Abteilung für die Gleichberechtigung der Geschlechter im Ministerium für Justiz, Gleichberechtigung und Rechtsreform, dessen Ziel in der Förderung von Initiativen im Bereich Frauen und Unternehmertum bestand. Der Pilotzyklus wurde 2009 durchgeführt, um eine größere Anzahl von Unternehmerinnen zu Wachstumsbestrebungen zu ermutigen und sie bei der Vergrößerung ihrer bestehenden Unternehmen sowie der Erreichung ihrer Wachstumsziele zu unterstützen. Die Initiative sollte die wirtschaftlichen, gesellschaftlichen und persönlichen Auswirkungen ihrer unternehmerischen Tätigkeit verstärken. Bislang wurden sechs Zyklen abgeschlossen.

\section{Aktivitäten}

Unternehmer bzw. Unternehmerinnen lernen oft am wirkungsvollsten voneinander. Entsprechend basiert Going for Growth auf interaktiven Gesprächsrunden, die nicht von Beratern, Wissenschaftlern oder professionellen Ausbildern moderiert werden, sondern von erfolgreichen Unternehmerinnen. Diese werden als „Leiterinnen“ bezeichnet. Dieser Ansatz bietet den Teilnehmerinnen eine einzigartige Lernumgebung unter der Leitung von Gleichgestellten und basiert auf dem Erfahrungsaustausch seitens der Leiterin und der anderen Teilnehmerinnen, die vor identischen Herausforderungen stehen. 
Going for Growth ist handlungs- und ergebnisorientiert. Es basiert auf der Erfahrung dessen, was im wirklichen Leben funktioniert oder nicht funktioniert hat. Die Teilnehmerinnen werden dabei unterstützt, die Entwicklung ihrer Unternehmen strategisch anzugehen, indem sie sich auf Wachstumsziele und zeitlich definierte Meilensteine konzentrieren.

\section{Leiterinnen}

Going for Growth nutzt die Beiträge erfolgreicher Unternehmerinnen, die als „Leiterinnen“ auftreten. Aktuell wirken 28 Leiterinnen an der Initiative mit, darunter zwei ehemalige Teilnehmerinnen. Jede von ihnen widmet dem Projekt freiwillig ihre Zeit. Die wichtigste Eigenschaft der Leiterinnen ist ihre Erfahrung mit der Vergrößerung eines Unternehmens. Außerdem verstehen sie es, ihre Erfahrung auf andere Unternehmen anzuwenden, haben ein offenes Ohr und verfügen über ausgezeichnete Kommunikationsfähigkeiten. Der Projektdirektor und erfahrene Leiterinnen helfen neuen Leiterinnen bei der Vorbereitung auf ihre Funktion und schulen sie in den Methoden von Going for Growth.

Bei Going for Growth stehen moderierte Gruppensitzungen im Zentrum, bei denen die Leiterinnen ihr Wissen und ihre Erfahrungen mit der Gruppe teilen. Die Leiterinnen haben auch die Funktion von Rollenvorbildern - sie sollen zeigen, dass eine Frau neben der Familie und anderen Verpflichtungen ein erfolgreiches Unternehmen aufbauen kann. Obwohl die Teilnehmerinnen keinen Einfluss auf die Auswahl der Leiterin haben, die ihre Gruppe moderiert, zeigen Feedback-Umfragen unter den Teilnehmerinnen, dass mehr als $90 \%$ von ihnen ihre Leiterin als für sie geeignet betrachteten. Zwischen dem zweiten und dem sechsten Projektzyklus lagen die angegebenen Zufriedenheitsquoten bei $90 \%, 92 \%, 98 \%, 96 \%$ und $98 \%$.

\section{Teilnehmerprofil}

Bislang nahmen an jedem der Zyklen jeweils 60 Frauen aus allen Regionen des Landes teil. Der Schwerpunkt liegt auf einer kleinen Anzahl engagierter Teilnehmerinnen, die eine klare Wachstumsbestrebung haben, die Möglichkeit zu schätzen wissen und sich zur Teilnahme an allen Gesprächsrunden und dem Nationalen Forum (siehe unten) verpflichten.

Um als Kandidatinnen für das Programm infrage zu kommen, müssen die Unternehmerinnen bereits geschäftsführende Inhaber eines Unternehmens sein, bei dem sie Hauptanteilseigner sind und für das sie hauptverantwortlich Entscheidungen treffen. Außerdem muss das Unternehmen seit mindestens zwei Jahren aktiv sein. Die Unternehmerinnen müssen zudem in Vollzeit für das Unternehmen tätig sein. Vor Kurzem kamen ausnahmsweise eine kleinere Anzahl von Bewerbungen von Kandidatinnen, die seit weniger als zwei Jahren Umsätze erzielen, in Betracht, wenn die neuen Unternehmen als besonders innovativ galten und ein erhebliches Wachstumspotenzial besaßen.

Um sich für das Programm zu bewerben, füllen die Kandidatinnen ein Bewerbungsformular aus, auf dem sie ihre aktuelle Stellung in Bezug auf Umsatz, Zahl der Beschäftigten und Marktorientierung beschreiben und angeben, wo sie in fünf Jahren sein möchten. Sie werden gefragt, was sie momentan am Wachstum hindert, wie die Teilnahme an der Initiative ihnen ihrer Ansicht nach helfen würde und was sie im Verlauf des Zyklus als Erfolg betrachten würden. Anschließend beschreiben sie in höchstens 500 Wörtern die bisherige Entwicklung ihres Unternehmens und ihre Vision für sein Wachstum und seine Entwicklung in der Zukunft. Schließlich skizzieren sie in höchstens 250 Wörtern die Gründe, warum sie Unternehmerin geworden sind.

Es gehen mehr Bewerbungen ein, als Plätze verfügbar sind. Daher findet unter Mitwirkung der Leiterinnen ein Auswahlverfahren statt. Die Leiterinnen prüfen gemeinsam die Bewerbungen, und jede von ihnen wählt acht Unternehmerinnen aus, die sie zu ihrer Gesprächsrunde einladen möchte. Die Aufnahme in das Programm basiert auf dieser schriftlichen Bewerbung; es findet kein Gespräch statt.

\section{Die Gesprächsrunden}

Eine gemeinsame Agenda, bei der Wachstum im Mittelpunkt steht, dient bei jeder der im Laufe des Zyklus stattfindenden Gesprächsrunden als roter Faden. Die Leiterin teilt ihre Erfahrungen und bringt ihre Gedanken zur jeweils besprochenen Frage zum Ausdruck. In ähnlicher Weise tauschen die anderen 
Teilnehmerinnen Erfahrungen aus und lernen voneinander. Die Leiterinnen tragen entscheidend dazu bei, das Vertrauen zu stärken, das sich in der Gruppe entwickelt. Die Gesprächsrunden finden einmal monatlich von Januar bis Juni statt. Alle besprochenen Themen werden vertraulich behandelt.

\section{Going for Growth-Website}

Die Website von Going for Growth (www.goingforgrowth.com/) bietet umfassende Informationen zu dem Programm und dem Zugang dazu. Sie enthält Informationen zu den verschiedenen Projekten von Going for Growth. In einem Mitgliederbereich wird die Netzwerkarbeit gefördert.

\section{Das Nationale Forum}

In der Mitte des Projektzyklus wird ein Nationales Forum abgehalten, an dem die Teilnahme aller Teilnehmerinnen erwartet wird. Das Nationale Forum soll den Teilnehmerinnen und den Leiterinnen Gelegenheit geben, sich eine Reihe von Themen zu erarbeiten, die alle Gesprächsrunden als wichtig für ihre aktuelle Situation erachten und die sie näher untersuchen möchten. Auf Präsentationen zu bestimmten Themen folgen von den Leiterinnen moderierte Gespräche unter den Gesprächsrunden. Bei diesen Gesprächen steht die Anwendung der aus den verschiedenen Präsentationen gewonnenen Erkenntnisse im Vordergrund.

Das Nationale Forum geht mit einem bestimmten Zyklus einher und beschränkt sich auf die Teilnehmerinnen dieses Zyklus. Die Anzahl der Teilnehmerinnen an dem Forum bewegt sich daher bei etwa 70, da zu den Teilnehmerinnen noch deren Leiterinnen hinzukommen. Am Spätnachmittag schließen sich auch Vertreter der Sponsoren an und bleiben zum Abendessen. Im Herbst wird außerdem ein Gemeinschaftsforum ausgerichtet. Die Teilnehmerzahlen sind dabei höher, da das Gemeinschaftsforum dem gesamten Plenum der Leiterinnen und allen ehemaligen Teilnehmerinnen offensteht, die Mitglied der Going-for-Growth-Gemeinschaft sind.

Im nationalen Forum können Schlüsselthemen ausführlicher behandelt werden, und die Teilnehmerinnen erhalten die Gelegenheit zur Netzwerkarbeit mit den Teilnehmerinnen und Leiterinnen anderer Gesprächsgruppen.

\section{Überwachung und Evaluierung}

Laufende Überwachung und Evaluierung ist fester Bestandteil der Struktur von Going for Growth. Sowohl die Leiterinnen als auch die Teilnehmerinnen wirken aktiv an der Bereitstellung von ausführlichen Informationen und Rückmeldungen während des gesamten Zyklus sowie bei Abschluss dessen mit.

Nach dem Anwerbe- und Auswahlverfahren wird ein Bericht angefertigt, der eine ausführliche Analyse der ausgewählten Kandidatinnen im Hinblick auf aktuellen Umsatz, Zahl der Beschäftigten und Exporttätigkeit enthält. Ihre Bestrebungen in Bezug auf jeden dieser Aspekte werden ausführlich angegeben. Diese werden mit den früheren Zyklen verglichen. Auch die Wachstumshindernisse, die die Teilnehmerinnen in ihren Bewerbungsformularen angegeben haben, werden beleuchtet. Dieser Bericht wird an die Leiterinnen und an die Sponsoren weitergegeben. Jede Leiterin erhält zudem eine ausführliche Analyse der Teilnehmerinnen ihrer Gesprächsrunde und der Bestrebungen dieser Teilnehmerinnen.

Am Ende des Zyklus wird jede Leiterin über die Fortschritte, die die Teilnehmerinnen ihrer Runde insgesamt erzielt haben, im Vergleich zur gesprächsrundenübergreifenden Norm informiert. Von jeder der Leiterinnen wird ein individuelles Feedback eingeholt. Die Leiterinnen treffen sich außerdem untereinander, um zu besprechen, was in dem jeweiligen Zyklus gut gelaufen ist und welche Aspekte verbesserungsbedürftig sind.

Am Ende jedes Zyklus wird aus allen gesammelten Informationen ein ausführlicher Analysebericht erstellt. Die qualitativen Ergebnisse werden skizziert. Da am Anfang und am Ende des Zyklus Informationen zum Umsatz, zur Zahl der Beschäftigten und zur Exporttätigkeit erfasst werden (d. h. eigene Angaben), können in Bezug auf diese Ergebnisse konkrete Auswirkungen festgestellt werden. Unterschiede zwischen den Zyklen werden festgehalten und Empfehlungen für künftige 
Zyklen ausgesprochen. Dies wird den Leiterinnen mitgeteilt, die an dem jeweiligen Zyklus beteiligt waren, und an die Sponsoren weitergegeben.

\section{Projektentwicklung}

Von Beginn an machte die gegenseitige Unterstützung von Unternehmern das zentrale Element von Going for Growth aus. Die in der Pilotphase umgesetzten Schlüsselelemente sind relativ gleich geblieben, doch im Laufe der Jahre hat sich mit zunehmender Bekanntheit von Going for Growth die Anzahl der Bewerberinnen für die Initiative stark erhöht. Es gibt nun einen viel stärkeren Wettbewerb um Plätze als noch in den Anfangsjahren.

Auf der Grundlage von Rückmeldungen von früheren Teilnehmerinnen und von den Leiterinnen wurden ergänzende Initiativen entwickelt, die kontinuierliche, für die Entwicklungsstufe der jeweiligen Unternehmerin angemessene Unterstützung bieten.

- Entwicklung einer Going for Growth-Gemeinschaft: Bei erfolgreichem Abschluss eines Zyklus erhalten die Teilnehmerinnen nun die Gelegenheit, miteinander in Kontakt zu bleiben und weiterhin das Wachstum ihres Unternehmens zu fördern, indem sie Mitglied der Going for Growth-Gemeinschaft werden. Die Mitglieder der Gemeinschaft können an einer Gesprächsrunde mit dem Titel Die Dynamik aufrechterhalten teilnehmen, themenspezifische Workshops besuchen und werden zu einem einmal jährlich im Herbst stattfindenden Gemeinschaftsforum eingeladen. Ein spezieller Bereich der Website wurde entwickelt, um den Mitgliedern die Kommunikation und die Netzwerkarbeit zu erleichtern. Die Teilnehmerinnen zahlen eine kleine Mitgliedschaftsgebühr (300 EUR im Jahr 2015), und die Gemeinschaft steht kurz davor, sich selbst tragen zu können.

- Gesprächsrunde der Leiterinnen: Da die Leiterinnen den Erfolg der Gesprächsrunden bei der Förderung des Unternehmenswachstums bei den Teilnehmerinnen aus erster Hand miterlebt haben, entschlossen sie, eine Gesprächsrunde einzurichten, mit der sie ihre gegenseitige Unterstützung strukturiert fördern können, um das Wachstum ihrer Unternehmen anzukurbeln. Diese Treffen finden dreimal jährlich statt.

- Die Initiative Starker Einstieg ist die neueste einer Reihe ergänzender Initiativen und richtet sich an ehrgeizige Unternehmerinnen, die sich am Anfang der Entwicklung ihres Unternehmens befinden. Sie wurde während des 6. Zyklus entwickelt und getestet (2014). Die anvisierten Unternehmen befinden sich in der Regel in der Phase, in der sie erst seit Kurzem oder noch keine Einnahmen genieren, doch sind sie schon weit über die Konzeptionsphase hinaus gediehen. „Starker Einstieg“ zielt auf Unternehmerinnen ab, bei denen die Dauer der Entwicklungsphase, bevor Einnahmen generiert werden, der Innovationsgrad und das Wachstumspotenzial deutlich über dem Durchschnitt liegen. Der Ansatz des gegenseitigen Lernens unter Gleichgestellten weist Ähnlichkeiten mit dem von Going for Growth auf, wurde jedoch angepasst, um den besonderen Bedürfnissen derer Rechnung zu tragen, die sich in einer frühen Entwicklungsphase befinden. Vor Kurzem konnten Sponsoren aus dem Unternehmenssektor gesichert werden, um zusätzliche maßgeschneiderte Unterstützungsleistungen wie z. B. Workshops anbieten zu können. Derzeit nehmen 24 Frauen an den drei Gesprächsrunden von „Starker Einstieg“ teil.

\section{Projektfinanzierung}

Die durchschnittlichen Kosten pro Teilnehmerin und Zyklus betragen bei Going for Growth weniger als 2000 EUR für einen sechsmonatigen Zyklus. Die Kosten der Initiative sind so niedrig, weil die Leiterinnen ihre Zeit freiwillig beisteuern und ihre eigenen Kosten tragen. Die Unternehmerinnen zahlen für die Teilnahme an Going for Growth keine Gebühr.

Going for Growth wird seit seinem Beginn durch die Maßnahme Equality for Women (Gleichstellung für Frauen) und durch Enterprise Ireland, die für die Entwicklung und das Wachstum irischer Unternehmen verantwortlichen Regierungsorganisation, finanziert. Über den Zeitraum von 2010 bis einschließlich 2014 hinweg erhielt das Projekt durchschnittlich 48000 EUR pro Jahr von der Maßnahme Equality for Women, die über das operationelle Programm für Investitionen in das Humankapitel durch den Europäischen Sozialfonds und das Ministerium für Justiz und Gleichstellung finanziert wird. Im Jahr 2015 wurde auch KPMG ein Sponsor. 


\section{Herausforderungen}

Eine Herausforderung ist die Sicherstellung einer Finanzierung für die Initiative. Going for Growth wurde zu Beginn der Wirtschaftskrise und der anschließenden Rezession konzipiert und entwickelt. Die öffentlichen Haushalte waren stark eingeschränkt. Die Initiative stützt sich weitgehend auf öffentliche Mittel, doch das Wachstum des Programms überstrapaziert einen ohnehin kleinen Haushalt. Seit 2015 ist der Unternehmenssektor stärker an der finanziellen Unterstützung beteiligt, was die Entwicklung der ergänzenden Initiativen erleichtert.

Eine weitere Herausforderung bestand darin, eine Kohorte von ehrgeizigen Unternehmerinnen zu finden. In Irland gibt es viele Netzwerke von Unternehmerinnen und Geschäftsfrauen. Daher war es schwierig, das Projekt von anderen abzuheben und Frauen mit einem angemessenen Ehrgeiz anzuziehen. Der Ruf der Leiterinnen spielte eine wichtige Rolle dabei, zu Beginn für Glaubwürdigkeit zu sorgen und Interesse an der Initiative zu erwecken, um eine geeignete Auswahl von Kandidaten zu einer Bewerbung zu ermutigen. Nun, da sich Going for Growth nach und nach einen Ruf erarbeitet hat und mehr in verschiedenen Medien über das Programm berichtet wird, melden sich während des ganzen Jahres Frauen auf der Website an. Die immer mehr werdenden ehemaligen Teilnehmer sind Botschafter für die Initiative, da sie selbst den Nutzen der Teilnahme erfahren haben. In den letzten Jahren haben sich die Anzahl und die Qualität der Bewerbungen sowie der Ehrgeiz unter den Bewerberinnen verglichen mit der Anfangsphase des Projekts erhöht.

Die Leiterinnen sind zwar von wesentlicher Bedeutung für das Projekt, stellen jedoch auch eine doppelte Herausforderung dar: Einerseits muss ihre Anzahl und ihr Kaliber, andererseits ihre Mitwirkung und Verpflichtung aufrechterhalten werden. Als das Pilotprojekt geplant wurde, wandte man sich an Unternehmerinnen mit ausgezeichnetem Ruf und Profil. Ihr nachhaltiges Engagement setzt Maßstäbe für andere Freiwillige, die angesprochen werden. Mit der Zeit wurde offensichtlich, dass die Leiterinnen von den Fortschritten begeistert sind, den sie bei den Teilnehmerinnen im Laufe des Zyklus beobachten. Sie sind maßgeblich an der Überprüfung und Weiterentwicklung der Initiative beteiligt und haben unter sich ein äußerst starkes Netzwerk aufgebaut.

\section{Auswirkungen}

Going for Growth befindet sich nun in seinem 7. Zyklus. Mehr als 400 ehrgeizige Unternehmerinnen wurden unterstützt, um damit ihre Ziele anzuheben und ihre Fähigkeit zu stärken, ein bedeutendes Unternehmenswachstum zu erreichen. Berichte der Teilnehmerinnen bestätigen den Nutzen, der aus dem Programm gezogen wird.

Ausgehend von Rückmeldungen und Berichten der Klienten geben die Leiter von Going for Growth an, dass dieTeilnehmerinnen von einer größeren Motivation, einer besseren strategischen Positionierung und einer verminderten psychologischen Isolation profitieren. Am Ende des sechsten Zyklus gaben 93\% der Teilnehmerinnen an, sie fühlten sich der Erreichung ihrer Wachstumsbestrebungen näher; $95 \%$ gaben an, die Gesprächsrunden hätten zu praktischen Veränderungen in ihrem Unternehmen geführt; $100 \%$ beabsichtigten, mit den anderen Teilnehmerinnen in ihrer Gruppe in Kontakt zu bleiben; und $100 \%$ würden das Programm weiterempfehlen.

Die Auswirkungen sind auch in Form von höheren Umsätzen, zusätzlich geschaffenen Arbeitsplätzen und anhand erstmaliger Exporttätigkeiten messbar. Mehr als 70\% der Teilnehmerinnen, die den sechsten Zyklus abgeschlossen haben, verzeichneten während des sechsmonatigen Zeitraums einen Umsatzanstieg. Durchschnittlich lag das Wachstum bei 15\%. Während dieses Zeitraums wurden zusätzlich 88 Mitarbeiter eingestellt. Dies entspricht einem Anstieg von insgesamt 13\%. Außerdem wurden zehn Praktikanten eingestellt. Die Zahl der Exporteure unter den Teilnehmerinnen stieg um $22 \%$.

Seit der Pilotphase stellt Going for Growth im Rahmen seines Ziels, Unternehmerinnen (unabhängig von einer Teilnahme an der Initiative) zu mehr Ehrgeiz zu ermutigen, seit vielen Jahren eine bedeutende Werbekampagne unter Mitwirkung der Leiterinnen und der Teilnehmerinnen auf die Beine. Durch ihre Handlungen zeigen diese Unternehmerinnen, dass ein Wachstum möglich ist, und übernehmen so für andere die Funktion von Rollenvorbildern. Sie erscheinen nicht nur in 
den traditionellen und digitalen Medien auf nationaler und lokaler Ebene, sondern stehen auch für Vorträge bei diversen Veranstaltungen zur Verfügung, zu denen sich Unternehmerinnen versammeln.

Going for Growth wurde 2015 in der Kategorie „Investition in Fähigkeiten“ mit dem Europäischen Unternehmensförderpreis ausgezeichnet.

\section{Voraussetzungen für die Übertragung}

Going for Growth ist bereits auf andere Kontexte übertragen worden (siehe Textfeld 12.1).

\section{Textfeld 12.1. Die Pilotgesprächsrunde von Going for Growth 2014 in Finnland}

Going for Growth wurde 2014 mit einer Gesprächsrunde als Pilotprojekt in Finnland durchgeführt. Dieses Pilotprojekt wurde durch den Europäischen Sozialfonds, die Zentren für Wirtschaftsentwicklung, Verkehr und Umwelt und die Universität Oulu finanziert. Anne Berner, Präsidentin des Verwaltungsrates von Oy Vallila Interior Ab, übernahm die Rolle der freiwilligen Leiterin.

Die Leiterin und acht Teilnehmerinnen aus Finnland nahmen zusammen mit den Teilnehmerinnen und Leiterinnen des 6. Zyklus aus Irland am Nationalen Forum von Going for Growth im März teil. Aus der Befragung am Ende des Zyklus lässt sich klar ablesen, dass alle finnischen Teilnehmerinnen ihre Teilnahme als sehr nutzbringend betrachteten. Im Verlauf des Zyklus steigerten $50 \%$ von ihnen ihre Umsätze um durchschnittlich 14,6\% und erhöhten die Zahl ihrer Beschäftigten um 9\%. Alle gaben an, sie fühlten sich infolge ihrer Teilnahme der Erreichung ihrer Wachstumsziele näher.

Die Erfahrung der Durchführung von Going for Growth in Finnland zeigt, dass die Initiative grenzübergreifend übertragen werden kann. Die Schlüsselfaktoren für eine erfolgreiche Übertragung dieser Initiative auf einen anderen Kontext sind folgende:

1. Einholung einer Anfangsfinanzierung, um die Projektstruktur einzurichten. Um für die Initiative zu werben, eine Kommunikationsstruktur aufzubauen und die Ausrichtung nationaler oder regionaler Foren zu ermöglichen, ist ein gewisses Maß an Finanzierung notwendig.

2. Einsetzung eines Projektkoordinators. Ein motivierter Koordinator ist entscheidend, um Leiterinnen $\mathrm{zu}$ identifizieren und zu gewinnen, die an der Initiative teilnehmen, Werbung für sie machen und die Entwicklung des Netzwerks unterstützen.

3. Identifizierung erstklassiger Leiterinnen. Die Mitwirkung erstklassiger Leiterinnen ist maßgeblich für den Erfolg des Programms. Es ist wichtig, Auswahlkriterien für die Leiterinnen zu entwickeln, um sicherzustellen, dass sie ein angemessenes Erfahrungs- und Erfolgsniveau haben. Dies sorgt dafür, dass das Programm für potenzielle Teilnehmerinnen attraktiv wird. Allerdings dürfen die Kriterien nicht so strikt sein, dass sie freiwillige Leiterinnen von einer Mitwirkung abschrecken.

4. Konzeption und Umsetzung eines angemessenen Auswahlverfahrens für Teilnehmer. Die irische Erfahrung zeigt, dass das Interesse an einer Teilnahme an der Initiative die Anzahl der verfügbaren Plätze übersteigt. Um die Auswirkungen zu maximieren, sollten die Teilnehmer anhand ihrer Motivation und des Wachstumspotenzials ihrer Unternehmen ausgewählt werden. Es ist wichtig, auch Unternehmerinnen aufzunehmen, die einem Wachstum offen gegenüberstehen, es aber noch nicht erreichen.

5. Einsatz eines oder mehrerer Champions zur Förderung des Programms. Die Initiative ist in Irland aus zwei Gründen so beliebt: Erstens übernehmen sowohl die Teilnehmerinnen als auch die Leiterinnen die Rolle von Champions und machen bei jeder sich ihnen bietenden Gelegenheit Werbung für die Initiative. Eine solche Unterstützung von den Projektbeteiligten trägt dazu bei, eine glaubwürdige Botschaft an potenziell an einer Teilnahme Interessierte auszusenden. Gleichzeitig profitiert die irische Initiative von einer umfassenden Presseberichterstattung. Daher ist es wichtig, dass ein Kommunikationsplan erstellt wird und zumindest einige der Bemühungen um Kontaktaufnahme der Champions koordiniert werden. 
6. Überwachung zum Zwecke der Analyse der Auswirkungen. Going for Growth führt eine genaue Überwachung der Geschäftsaktivitäten der Teilnehmerinnen durch und bemüht sich anhaltend um eine Verbesserung des Unterstützungsangebots. Dazu gehört eine informelle Überwachung sowie eine regelmäßige Umfrage am Ende jedes Zyklus. Es ist wichtig, die erzielten Fortschritte zu bewerten und die Ergebnisse an die Initiative zurückzumelden.

\section{Literatur}

Europäische Kommission (2003), „Unternehmergeist in Europa“, von der Europäischen Kommission vorgelegtes Grünbuch, Januar 2003 http://ec.europa.eu/transparency/regdoc/rep/1/2003/ DE/1-2003-27-DE-F1-1.Pdf

Global Entrepreneurship Monitor (2008), „GEM Report for Ireland 2008“, verfügbar unter: www.gemconsortium.org/docs/download/526 


\section{Invitalias Unterstützung für das Unternehmertum Arbeitsloser, Italien}

Diese Fallstudie stellt ein Beispiel für ein integriertes Unterstützungsprogramm für Arbeitslose vor, die sich für eine Unternehmensgründung interessieren, und erörtert die Ziele, den Hintergrund und die durchgeführten Aktivitäten. Außerdem werden Daten vorgestellt, die die Auswirkungen des Programms veranschaulichen. Es folgt eine Erörterung der Herausforderungen und der Voraussetzungen für eine Übertragung dieses Programms auf einen anderen Kontext.

\section{Ziele}

Das Ziel von Invitalias Maßnahme zur Unterstützung für das Unternehmertum Arbeitsloser besteht darin, sicherzustellen, dass alle Arbeitslosen die Möglichkeit erhalten, in das Unternehmertum einzusteigen. Arbeitslosen jungen Menschen wird beim Zugang Priorität eingeräumt und sie erhalten zusätzliche Unterstützung und Anreize. Auch Start-ups von Arbeitslosen in den Südregionen Italiens wird beim Zugang Priorität eingeräumt, da die Arbeitslosenquoten dort nach wie vor viel höher sind als in den zentralen und nördlichen Regionen.

Das Programm nutzt drei Wege, um Arbeitslose beim Einstieg in das Unternehmertum zu unterstützen: selbstständige Erwerbstätigkeit, Gründung von Kleinstunternehmen und Franchising. Existenzgründer unter den Arbeitslosen können in den folgenden Sektoren eine Unterstützung erhalten: Produktion, Dienstleistungen und Handel (die Landwirtschaft ist ausgeschlossen). Zu den Zielen des Programms gehören:

- Unterstützung der Arbeitslosen beim Einstieg in den Arbeitsmarkt;

- Förderung der Entwicklung des Unternehmertums auf lokaler Ebene;

- Verbreitung und Förderung einer Geschäftskultur;

- Stärkung der Fähigkeiten der Begünstigten, um ihre Beschäftigungsfähigkeit zu verbessern; und

- Förderung der Entwicklung einer Unternehmensinfrastruktur.

Allgemeiner gefasst besteht das Ziel des Programms darin, eine lokale, unterstützende Unternehmenskultur aufzubauen (Invitalia, 2014). Für den Erfolg von Existenzgründern wird eine förderliche Infrastruktur benötigt, um die unternehmerische Entwicklung zu stärken, zum Beispiel in Form eines Beziehungsnetzwerks, das Rat, Informationen, Finanzierung und andere Ressourcen bietet.

\section{Hintergrund}

Den Hintergrund dieser Maßnahme bilden die folgenden zwei Aspekte: Erstens liegt das allgemeine Prinzip einer Unterstützung für Existenzgründer in der Bekämpfung der Arbeitslosigkeit. Maßnahmen zur Unterstützung Arbeitsloser beim Einstieg in das Unternehmertum werden recht kontrovers diskutiert. So sind einige Autoren der Ansicht, derartige Maßnahmen führten zu geringwertigen Unternehmensgründungen (Greene et al., 2004). Jedoch stellte man durch empirische Belege aus einer Umfrage unter mehr als 3000 Existenzgründern aus zwei deutschen Beihilfeprogrammen für Arbeitslose zur Gründung neuer Unternehmen fest, dass die Überlebensraten erheblich höher liegen als diejenigen, die häufig zitiert werden: „Die Überlebensraten zweieinhalb Jahre nach der Unternehmensgründung sind recht hoch (etwa 70\%) und fallen für beide Programme und beide Geschlechter ähnlich aus“ (Caliendo et al., 2010).

Zweitens bestand (und besteht weiterhin) in Italien die Notwendigkeit, mehr Arbeitsplätze zu schaffen und Arbeitslose wieder in Arbeit zu bringen. Seit der jüngsten Wirtschaftskrise sind die Arbeitslosenquoten 
in Italien gestiegen und erreichten im November 2014 einen Höchststand von 13,4\% (ISTAT, 2015). Im zweiten Quartal 2015 erreichte die nationale Arbeitslosenquote in Italien 12,1\%. Allerdings verbirgt dieser nationale Durchschnitt eine besonders hohe Arbeitslosenquote unter jungen Menschen (15- bis 24-Jährige) von 41,1\% im zweiten Quartal 2015. Der nationale Durchschnitt verbirgt zudem erhebliche Unterschiede in den regionalen Arbeitslosenquoten: In den Südregionen liegen die Arbeitslosenquoten 6 bis 7 Prozentpunkte höher als in entsprechenden Regionen im Norden. Die höchsten Arbeitslosenquoten verzeichnen die Regionen Sizilien, Sardinien und Kampanien - im Februar 2014 lag die Arbeitslosenquote Siziliens bei 22\% (ISTAT, 2015).

\section{Aktivitäten}

Invitalia, eine nationale italienische Agentur, ist damit beauftragt, die Wettbewerbsfähigkeit Italiens, unter anderem in strategischen Sektoren, zu stärken, wobei der Schwerpunkt auf der Verbesserung der Wirtschaftsentwicklung in Italiens Südregionen liegt. Obwohl es auch zu Invitalias Aufgabe gehört, ausländische Direktinvestitionen (DI-Zuflüsse) zu fördern, hat sich die Agentur der Förderung des Wettbewerbsvorteils Italiens, der Steigerung der Innovationskraft und der lokalen industriellen Entwicklung sowie der Stärkung der lokalen Wettbewerbsfähigkeit verschrieben. Um diese allgemeinen Ziele der Wirtschaftsentwicklung und verbesserten Wettbewerbsfähigkeit zu erreichen, unterstützt sie Unternehmensgründungen mit besonderem Schwerpunkt auf Innovationen (Invitalia, 2014). Angesichts des nationalen Phänomens der hohen Jugendarbeitslosigkeit und regionaler Unterschiede in der Wirtschaftsentwicklung sind die Unternehmerprogramme von Invitalia weitgehend auf die Unterstützung der folgenden im Unternehmertum benachteiligten Gruppen ausgerichtet: junge Menschen bis 35 Jahre, Arbeitslose und Menschen aus einkommensschwachen Regionen in Süditalien.

Invitalia bietet eine Reihe von Programmen an, um Unternehmen und die Wirtschaftsentwicklung zu unterstützen, unter anderem für etablierte, wachstumsstarke und ausgereifte Unternehmen. In diesem Fallbeispiel liegt der Fokus auf Invitalias Unternehmerprogramm für Arbeitslose, das als Maßnahme für selbstständige Erwerbstätigkeit bekannt ist. Sie zielt darauf ab, Arbeitslose bei der Aufnahme einer selbstständigen Erwerbstätigkeit und bei der Gründung von Kleinstunternehmen, unter anderem neuer Franchise-Unternehmen, zu unterstützen. Zu den Kennzeichen des Programms gehören die finanzielle Unterstützung über „kostenlose“ Mikrokredite, Schulungen, Beratung und Informationen sowie individuelles Mentoring.

Das Programm wurde im November 1996 eingeführt und läuft noch heute. Dafür infrage kamen Menschen imAltervon mindestens 18Jahren und solche, die seit mindestens sechs Monaten arbeitslos waren. Zu den finanziellen Anreizen gehörten Beihilfen in Höhe von bis zu 25823 EUR für Investitionsausgaben und bis zu 5165 EUR für Betriebsmittelausgaben. Im Jahr 2000 wurde das Arbeitslosigkeitskriterium gelockert, sodass die Maßnahme auf alle (zum Zeitpunkt der jeweiligen Bewerbung für das Programm) Arbeitslosen gelten konnte, und die Maßnahme wurde auf alle Regionen Italiens ausgeweitet. Die im Rahmen des Programms geleistete Unterstützung wurde um Maßnahmen für Kleinstunternehmen und FranchiseUnternehmen erweitert.

Bei Invitalias Unternehmerprogramm für Arbeitslose konzentriert sich die Unterstützung auf das erste Jahr der Unternehmensgründung, wo das Risiko eines Scheiterns am größten ist. Während dieses entscheidenden Jahres der Unternehmensgründung werden bis zu einem Jahr lang unter anderem folgende intensive Dienstleistungen angeboten:

- Bewertung der Tragfähigkeit des Geschäftsvorschlags vor der Gründung, Durcharbeitung einer Bedarfsanalyse zusammen mit dem potenziellen Unternehmer; 
- Hilfe für den aufstrebenden Unternehmer beim Bewerbungsverfahren, um vor der Gründung in das Programm einzusteigen;

- Auszahlung finanzieller Anreize durch Beihilfen oder zinsfreie Darlehen;

- Managerschulungen, darunter Mentoring, Coaching und technische Unterstützung;

- Individuelles Mentoring, das die Entwicklung einer persönlichen Beziehung mit den Existenzgründern ermöglicht; und

- Online-Unterstützung, die für eine größere Reichweite des Programms in eher ländlichen und abgelegenen Regionen sorgt. Invitalia nutzt ein spezielles Online-Unterstützungsportal: www.autoimpiego.invitalia.it.

Die Überwachung des Programms erfolgt durch Invitalia. Dies schließt die Einhaltung der Auflagen für eine finanzielle Unterstützung seitens der Begünstigten ein, z. B. die Überwachung der Überlebensraten, die Rückzahlung von Darlehen und die Teilnahme an Schulungssitzungen.

$\mathrm{Zu}$ den Aktivitäten und Unterstützungsleistungen gehören Managerschulungen sowie technische und finanzielle Unterstützung. Es gilt zu bedenken, dass sich nicht jeder Mensch dazu eignet, durch die Aufnahme einer selbstständigen Erwerbstätigkeit in das Unternehmertum einzusteigen (Blackburn und Ram, 2006). Ein weiteres Ziel des Programms besteht daher darin, die Fähigkeiten der Begünstigten zu stärken, damit die Arbeitslosen ihre beruflichen Perspektiven auf dem italienischen Arbeitsmarkt verbessern. Tabelle 13.1 enthält eine ausführlichere Beschreibung der angebotenen Unterstützung sowie einen Vergleich der Aktivitäten in den drei Maßnahmen bzw. Kategorien selbstständige Erwerbstätigkeit, Kleinstunternehmen und Franchising.

\section{Tabelle 13.1. Invitalias Unterstützungsaktivitäten für das Unternehmertum Arbeitsloser}

\begin{tabular}{|c|c|c|c|}
\hline & Selbstständige Erwerbstätigkeit & Kleinstunternehmen & Franchising \\
\hline Sektoren & Produktion, Dienstleistungen und Handel & Produktion und Dienstleistungen & Handel und Dienstleistungen \\
\hline Max. Investitionssumme & 25823 EUR & 129114 EUR & Keine Begrenzung \\
\hline \multirow[t]{2}{*}{ Finanzielle Unterstützung (bis 2014) } & $\begin{array}{l}\text { Vollständige Deckung der Investitionen } \\
\text { im Rahmen der De-minimis-Grenze: } \\
\text { - Beihilfe } \\
\text { - Zinsverbilligtes Darlehen auf fünf } \\
\text { Jahre }\end{array}$ & $\begin{array}{l}\text { Vollständige Deckung der Investitionen } \\
\text { im Rahmen der De-minimis-Grenze: } \\
\text { - Beihilfe } \\
\text { - Zinsverbilligtes Darlehen auf fünf } \\
\text { Jahre }\end{array}$ & $\begin{array}{l}\text { Vollständige Deckung der Investitionen } \\
\text { im Rahmen der De-minimis-Grenze: } \\
\text { - Beihilfe } \\
\text { - Zinsverbilligtes Darlehen auf fünf } \\
\text { bis zehn Jahre }\end{array}$ \\
\hline & $\begin{array}{l}\text { Beihilfe zur Deckung der Betriebskosten } \\
\text { im ersten Jahr von bis zu } 5165 \text { EUR. }\end{array}$ & $\begin{array}{l}\text { Beihilfe zur Deckung der Betriebskosten } \\
\text { im ersten Jahr von bis zu } 5165 \text { EUR. }\end{array}$ & $\begin{array}{l}\text { Beihilfe zur Deckung der Betriebskosten } \\
\text { für die ersten drei Jahre. }\end{array}$ \\
\hline \multirow[t]{2}{*}{ Aktivitäten } & Geschäftsplanung & Geschäftsplanung & Geschäftsplanung \\
\hline & $\begin{array}{l}\text { Technische Unterstützung und } \\
\text { Unterstützung bei der Geschäftsführung }\end{array}$ & $\begin{array}{l}\text { Technische Unterstützung und } \\
\text { Unterstützung bei der Geschäftsführung }\end{array}$ & $\begin{array}{l}\text { Technische Unterstützung und } \\
\text { Unterstützung bei der Geschäftsführung }\end{array}$ \\
\hline
\end{tabular}

Quelle: Invitalia, 2014

Für alle drei Maßnahmen wählen die vor der Gründung stehenden Kandidaten die ersten zu erbringenden Dienstleistungen aus. Dazu gehören die Bewertung derTragfähigkeit des Geschäftsvorschlags, die Durcharbeitung einer Bedarfsanalyse zusammen mit dem Unternehmer und Hilfe für den aufstrebenden Unternehmer beim Bewerbungsverfahren, um in eine der drei Maßnahmen einzusteigen.

Es ist anzumerken, dass die Höhe der Unterstützungsbeihilfen der De-minimis-Grenze der Europäischen Union unterworfen war und dass man damit rechnete, dass nach einer Überarbeitung des Programms die Unterstützungsbeihilfe ab 2015 durch ein neues Programm mit zinsfreien Darlehen ersetzt werden würde. Workshops werden mit individuellen Treffen mit Bewerbern kombiniert, um sie bei der Entwicklung ihrer Geschäftsidee, ihres Geschäftsmodells und ihrer Geschäftspläne zu unterstützen.

Das Franchising wird in Zusammenarbeit mit akkreditierten Franchisegebern durchgeführt. Diese Maßnahme bietet Unterstützung bei der Unternehmensgründung für Menschen, die durch eine Investition in ein Franchise-Unternehmen in das Unternehmertum einsteigen möchten. Für einzelne Investitionen ist keine Grenze festgelegt, aber die Sektoren sind auf den Handel und Dienstleistungen beschränkt. 


\section{Projektmanagement}

Das Bewerbungsverfahren für das Programm gliedert sich in einzelne Stufen, die in Abbildung 13.1 dargestellt sind. In der ersten Phase geht es um Werbemaßnahmen für das Programm mit Seminaren zur Kontaktaufnahme und Workshops für Bewerber. In der zweiten Phase findet ein spezielles Evaluierungsverfahren für Programmbewerber statt. Die Bewerber sind verpflichtet, einen Geschäftsplan zu erstellen, der von Invitalia anhand einer Reihe von Kriterien bewertet wird, unter anderem:

- Stimmigkeit zwischen den Eigenschaften und Kompetenzen des Bewerbers und der Art des Geschäftsvorschlags;

- Angemessenheit der Marktbedingungen; und

- Tragfähigkeit des Vorschlags unter Berücksichtigung finanzieller, wirtschaftlicher und technischer Aspekte.

Lokale Partner übernehmen die Prüfung der Eignung der Bewerbung, die Beurteilung des Geschäftsplans und die Treffen mit den Kandidaten. Diese lokalen Partner sind lokale Entwicklungsagenturen und gehören zu den italienischen Regionalverwaltungen. Invitalia übernimmt jedoch den endgültigen Abschluss und die Genehmigung.

\section{Abbildung 13.1. Betriebsprozess, Verwaltung und Aktivitäten von Invitalias Programm zur Unterstützung des Unternehmertums von Arbeitslosen}

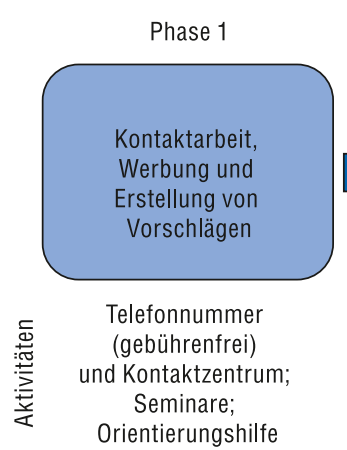

Quelle: Invitalia, 2014

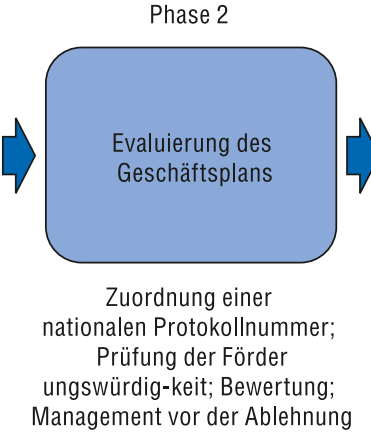

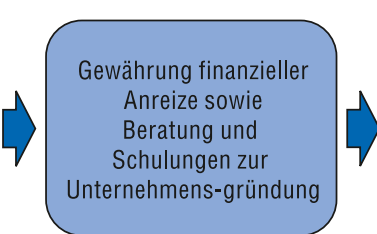

Auszahlung von Beihilfen und zinsverbilligten Darlehen; Seminare; Workshops; individuelles Mentoring; webbasierte Unterstützung
Phase 4

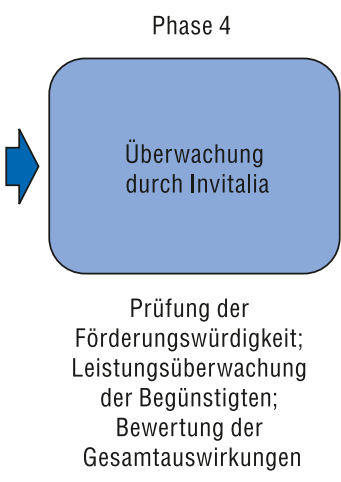

Sobald ein Bewerber von Invitalia genehmigt wird, steigt der Unternehmer nach einer Evaluierung seines Geschäftsplans in eine der drei Maßnahmen ein (Tabelle 13.1).

Somit werden den Begünstigten in Stufe 3 für ihre genehmigten Vorschläge finanzielle Anreize gewährt, unter anderem Beihilfen und bezuschusste Darlehen, die durch eine intensive Gründungsberatung durch individuelles Mentoring, Seminare und webbasierte Online-Unterstützung ergänzt werden. Außerdem wertet Invitalia die Auswirkungen des Programms durch eine dauerhafte Überwachung der finanzierten Unternehmen und die Erhebung von Daten dieser Unternehmen aus (Stufe 4). Dazu gehört eine Website zur selbstständigen Erwerbstätigkeit für Begünstigte, die eine Online-Unterstützung anbietet und die Erzeugung eines webbasierten Überwachungssystems auf der Basis eigener Angaben ermöglicht (www.Invitalia.autoimpiego.it).

\section{Projektfinanzierung}

Der Jahreshaushalt des Programms liegt durchschnittlich bei 120 Millionen EUR, abhängig von der Anzahl der eingereichten Vorschläge, deren Finanzierung genehmigt wurde. Im Haushalt sind Mittel für Beihilfen, zinsverbilligte Darlehen und technische Unterstützung inbegriffen. In den letzten acht Jahren wurde das Programm durch die nationale Regierung und den Europäischen Fonds für regionale Entwicklung finanziert. 


\section{Herausforderungen}

Das Programm wurde vor 17 Jahren eingerichtet. In der Zwischenzeit wurden vor dem Hintergrund der auftretenden Herausforderungen, der gezogenen Erkenntnisse und der gesammelten Erfahrungen Anpassungen und Änderungen vorgenommen.

Eine der Herausforderungen, mit denen man konfrontiert war, ist die große Anzahl der Bewerber während des gesamten Zeitraums, in dem das Programm bislang durchgeführt wurde. Kennzeichnend für diese Maßnahme ist ihre breit gefasste soziale Zielgruppe, bei der keine besonderen Prioritäten in Bezug auf das Geschlecht, die geschaffenen Arbeitsplätze oder die erzielten Umsätze bestehen. Darum blieb die Anzahl der Bewerbungen konstant hoch, selbst im Wirtschaftsklima nach der Krise. Die Anzahl der Bewerbungen betrug durchschnittlich 10000 pro Jahr im Zeitraum 2008-2011 und 5700 pro Jahr im Zeitraum 2012-2014 ( ${ }^{2}$ ). Dies hat zu einer Überstrapazierung der verfügbaren Ressourcen geführt, die das Unterstützungsniveau entsprechend einschränken. Die Seminare und Workshops sind beliebt und gut besucht. Bei der Ausweitung des Programms durch die Kleinstunternehmen- und die FranchisingMaßnahme wurden die Ressourcen zusätzlich beansprucht. Allerdings trug diese Ausweitung auch zur Erreichung beeindruckender Überlebensraten bei.

Neben der finanziellen Unterstützung und den Schulungen hat sich die Beratung der Bewerber durch individuelles Mentoring als wichtig für das Überleben der Unternehmen erwiesen und zu den beachtlichen Auswirkungen des Programms in Bezug auf die Schaffung von Arbeitsplätzen beigetragen.

Partnerschaften sind wichtig für die Kontaktaufnahme und Werbemaßnahmen für das Programm. Das Programm wird in Zusammenarbeit mit lokalen Entwicklungsagenturen (in Besitz der Regionen) verwaltet, die den Begünstigten technische Unterstützung leisten. Die Werbetätigkeiten, die auf die besonderen Bedürfnisse auf lokaler Ebene angepasst sind, werden zusammen mit verschiedenen lokalen Akteuren durchgeführt, unter anderem mit der italienischen Handelskammer.

\section{Auswirkungen}

Tabelle 13.2 enthält Informationen zu den Auswirkungen des Programms von 1996 bis 2013.

\section{Tabelle 13.2. Ergebnisse von Invitalias Programm zur Unterstützung des Unternehmertums von Arbeitslosen, 1996-2013}

\begin{tabular}{lc}
\hline \multicolumn{1}{c}{ Kriterien } & Ergebnis \\
\hline Eingereichte Bewerbungen $\left(^{*}\right)$ & 327280 \\
Neu gegründete Unternehmen & 111124 \\
Wert der Beihilfen und Darlehen & 4032,7 Millionen EUR \\
Zugewiesene Mittel & 5299 Millionen EUR \\
Geschaffene Arbeitsplätze & 193084 \\
\hline
\end{tabular}

Quelle: Diese Information legte Invitalia der OECD im Februar 2015 vor.

Hinweise: (*) Von den eingereichten Bewerbungen bezogen sich 278062 (85\%) auf eine selbstständige Erwerbstätigkeit, $45511(13,9 \%)$ auf ein Kleinstunternehmen und $3624(1,1 \%)$ auf ein Franchising. Die Bewerbungen für Kleinstunternehmen und Franchising sind für den Zeitraum 2003-2013 angegeben.

Eine weitere Aufschlüsselung der Merkmale der Programmteilnehmer findet sich in Tabelle 13.3. Es zeigt sich, dass die Begünstigten überwiegend junge Menschen unter 36 Jahren mit relativ gutem Bildungshintergrund (mit einem Sekundarschul- oder höherem Abschluss) waren und dass ein beachtlicher Anteil Frauen waren, die in dem Programm gut repräsentiert sind.

2 Diese Information legte Invitalia der OECD im Februar 2015 vor. 


\section{Tabelle 13.3. Persönliche Merkmale der Teilnehmer an Invitalias Programm zur Unterstützung des Unternehmertums von Arbeitslosen}

\begin{tabular}{ll}
\hline \multicolumn{1}{c}{ Merkmal } & Prozentsatz \\
\hline $\begin{array}{ll}\text { Geschlecht } \\
\text { Männlich }\end{array}$ & 56 \\
Weiblich & 44 \\
Alter & \\
$18-25$ & 21 \\
$26-35$ & 38 \\
$36-45$ & 26 \\
$46+$ & 15 \\
Bildung & \\
Grundbildung & 26 \\
Sekundarschulabschluss & 57 \\
Hochschulabschluss & 17 \\
\hline
\end{tabular}

Quelle: Diese Information legte Invitalia der OECD im Februar 2015 vor.

Die Überlebensraten sieben Jahre nach Unternehmensgründung werden mit $75 \%$ angegeben (Invitalia, 2014) - eine beeindruckende Überlebensrate für ein Programm, das das Ziel verfolgt, Arbeitslose beim Einstieg ins Unternehmertum zu unterstützen. Wie zuvor bereits erwähnt liegen die Misserfolgsquoten während des ersten Betriebsjahres neuer Unternehmen, die von vormals Arbeitslosen gegründet wurden, häufig bei 33\% oder höher (Greene et al., 2004). Die offiziellen Zahlen zum Scheitern von Unternehmen können irreführend sein, da sie alle Gewerbeabmeldungen berücksichtigen. Allerdings stellen diese nicht unbedingt alle einen Misserfolg dar; der Unternehmer hat sich unter Umständen zur Schließung eines tragfähigen Unternehmens entschlossen, um wieder in den Arbeitsmarkt einzusteigen.

\section{Voraussetzungen für die Übertragung}

Die Maßnahme für selbstständige Erwerbstätigkeit ist ein nationales Programm, das in allen Regionen Italiens umgesetzt wird. Somit handelt es sich um ein groß angelegtes Programm. Invitalia hat mit der Zeit ein Profil aufgebaut und Partnerschaften geschaffen, um das Programm durchzuführen. Die Bereitstellung von Programmen zur Unterstützung des Unternehmertums von Arbeitslosen geht mit zahlreichen Herausforderungen einher, insbesondere beim Herausfiltern geeigneter Bewerber und bei der Sicherstellung der Tragfähigkeit der Vorschläge. Wenn politische Entscheidungsträger Überlegungen zur Übertragbarkeit der Grundsätze des Programms anstellen, sollten sie eine Reihe von zentralen Erfolgsfaktoren berücksichtigen. Dazu gehören:

1. Vorgelagerte Unterstützung. Dabei wird den Bewerbern Hilfestellung bei der Erstellung von Geschäftsplänen und bei ihren geschäftlichen und unternehmerischen Fähigkeiten geleistet. Es ist wichtig zu gewährleisten, dass die Geschäftsvorschläge zu den Kompetenzen der Bewerber für das Programm passen.

2. Bereitstellung einer Kombination aus Finanz- und Beratungsunterstützung. Eine finanzielle Unterstützung kann eine wichtige Funktion als Anreiz erfüllen, damit die Bewerber an dem Programm teilnehmen und sich tragfähige Geschäftsideen einfallen lassen. Allerdings muss sie durch eine Beratung ergänzt werden, für die wahrscheinlich ein individuelles Mentoring vonnöten ist. Da Mentoring mit hohen Kosten verbunden sein kann, ist es wichtig, diejenigen Bewerber herauszufiltern, die es benötigen.

3. Entwicklung starker Partnerschaften. Partnerschaften dank der Zusammenarbeit mit lokalen Entwicklungsagenturen (in Besitz der Regionen) sind maßgeblich für die Kontaktarbeit und die Zugänglichkeit für potenzielle Kandidaten aus den Reihen der Arbeitslosen. Ein so entstehendes 
Netzwerk spielt eine wichtige Rolle bei der konsequenten nationalen Bereitstellung der Unterstützungsleistungen.

4. Nutzung von Technologien. Die integrierte und vollständig interaktive Website ist eine wichtige Entwicklung, um ein groß angelegtes Programm zugänglich zu machen. Sie ermöglicht Invitalia mehr Effizienz bei der Zuweisung der Ressourcen, die dem Programm gewidmet sind. Die Anzahl der Bewerbungen ist zwar gestiegen, doch kann das Programm die erhöhte Anzahl und die größere Bandbreite der Bewerber weiterhin bewältigen.

\section{Literatur}

Blackburn, R. und M. Ram (2006), „Fix or fixation? The contributions and limitations of entrepreneurship and small firms to combating social exclusion“, Entrepreneurship and Regional Development, Bd. 18, Nr. 1, S. 73-89.

Caliendo, M. und A. Kritikos (2010), „Start-ups by the unemployed: characteristics, survival and direct employment effects“, Small Business Economics, Bd. 35, S. 71-92.

Greene, F., K. Mole und D. Storey (2004), „Does more mean worse? Three decades of enterprise policy in the Tees Valley“, Urban Studies, Bd. 41, Nr. 7, S. 1207-1228.

Invitalia (2014), Invitalia-Website, verfügbar unter: www.invitalia.it (Zugriff am 23. Oktober 2014).

ISTAT (2015), Italienisches Nationales Institut für Statistik, Pressemeldung, Januar 2015, verfügbar unter: www.istat.it/en/archive/PressRelease (Zugriff am 9. Februar 2015).

ISTAT (2014), „Employment and unemployment: provisional estimates, August 2014“, Italienisches Nationales Institut für Statistik, verfügbar unter www.istat.it/en/files/2014/04/201402_PressRelease (Zugriff am 8. Oktober 2014).

SviluppoBasilicata (2015), Einrichtungen von Invitalia für selbstständige Erwerbstätigkeit, verfügbar unter: www.suiluppobasilicata.it (Zugriff am 20. Februar 2015). 


\section{Massnahme zur Aufnahme einer gewerblichen Tätigkeit oder selbstständigen Erwerbstätigkeit, Lettland}

In dieser Fallstudie wird ein integriertes Programm vorgestellt, das Arbeitslose durch Schulungen, Beihilfen und eine Zuwendung bei der Unternehmensgründung unterstützt. Diese Beschreibung stellt die Ziele, die Hintergründe und die im Rahmen dieses Projekt durchgeführten Aktivitäten vor. Außerdem werden die bei der Durchführung des Projekts sichtbar werdenden Herausforderungen und die notwendigen Voraussetzungen für die Übertragung dieses Projekts auf einen anderen Kontext erörtert.

\section{Ziele}

Dieses Projekt wurde 2008 als Pilotprojekt ins Leben gerufen und läuft bis heute. Es zielt darauf $\mathrm{ab}$, qualifizierte Arbeitslose durch Unternehmensberatungsdienste, eine kleine Beihilfe und ein Überbrückungsgeld bei der Unternehmensgründung zu unterstützen. Das Ziel ist es, sicherzustellen, dass die von Arbeitslosen geführten Start-ups mindestens zwei Jahre lang überleben.

Um im Rahmen dieser Maßnahme eine Unterstützung in Anspruch nehmen zu können, müssen potenzielle Kandidaten bei der staatlichen Arbeitsagentur (Nodarbinātības valsts aǵentūra, NVA) arbeitslos gemeldet sein und über betriebswirtschaftliche Kenntnisse und Erfahrungen verfügen oder eine der folgenden Bildungsvoraussetzungen erfüllen:

- Sie verfügen über eine berufliche Sekundarausbildung oder Hochschulbildung im Bereich Betriebswirtschaft bzw. haben in dem Bereich abgeschlossen, in dem sie eine gewerbliche oder selbstständige Erwerbstätigkeit aufzunehmen beabsichtigen;

- sie haben in einer Berufsausbildung Qualifikationen erworben, die die im Bereich Betriebswirtschaft erforderlichen Kenntnisse vermitteln, oder

- sie verfügen über eine berufliche Sekundarausbildung oder Hochschulbildung und haben ein informelles (von der NVA angebotenes) Ausbildungsprogramm in einem mit Betriebswirtschaft zusammenhängenden Bereich abgeschlossen. 


\section{Hintergrund}

Die NVA hat dieses Pilotprojekt ins Leben gerufen, um sein Potenzial zu testen, Arbeitslose durch eine selbstständige Erwerbstätigkeit wieder in Arbeit zu bringen. Die Arbeitslosigkeit in Lettland stieg nach Beginn der Krise drastisch und stellte eine Belastung für die öffentlichen Arbeitsverwaltungen dar. Die Arbeitslosenquote erreichte 2010 einen Höchststand von 19,8\%. Der relative Anstieg der Arbeitslosenquote nach Beginn der Wirtschaftskrise fiel in Lettland viel höher aus als im Durchschnitt der Europäischen Union. 2009 setzte die lettische Regierung eine neue Vision für die Unterstützung von selbstständig Erwerbstätigen und Kleinstunternehmen in die Tat um. Zusätzlich zu der Fortführung der verringerten Einkommensteuersätze und der Sonderbedingungen für die Zahlung von Sozialversicherungsbeiträgen durch selbstständig Erwerbstätige wurden Änderungen am Unternehmensrecht vorgenommen, um das Regelungsumfeld für Kleinstunternehmen günstiger zu gestalten.

\section{Aktivitäten}

Diese Maßnahme bietet Unterstützung in zwei Phasen. Zunächst nehmen die Teilnehmer an einer Reihe von Beratungsterminen teil, bei denen sie individuelle Unterstützung bei der Vorbereitung und Entwicklung eines Geschäftsplans erhalten. Jeder Teilnehmer kann innerhalb von sechs Wochen an bis zu 20individuellen Beratungssitzungen teilnehmen.Das Hauptaugenmerk derSitzungenliegtaufderErmittlung nachhaltiger Geschäftsideen und dem Entwurf eines Plans auf dieser Grundlage. Diese Beratungssitzungen werden von Geschäftsexperten durchgeführt, die von der NVA unter Vertrag genommen werden. Sobald die Beratungstermine abgeschlossen sind und die Teilnehmer einen Geschäftsplan entwickelt haben, können sie sich für die zweite Unterstützungsphase bewerben, in der ihnen eine zusätzliche individuelle Unternehmensberatung und finanzielle Beihilfen angeboten werden.

DieNVA evaluiert die Bewerber, bevor diese eine intensivere Unterstützungerhalten, um sicherzustellen, dass deren Projekte realisierbar sind. Diese Evaluierungen werden von einem Ausschuss vorgenommen, der aus Branchenexperten und Experten im Bereich Unternehmensgründung und -entwicklung besteht. Der Ausschuss prüft das Potenzial der Geschäftspläne und die Wahrscheinlichkeit, mit der der Unternehmer diese erfolgreich umsetzt. Die wichtigsten Faktoren für ihre Entscheidung sind die Einzigartigkeit und der Innovationsgrad des Produkts bzw. der Dienstleistung und die potenzielle Nachfrage nach dem Produkt bzw. der Dienstleistung.

Projekte, die für die zweite Unterstützungsstufe ausgewählt werden, können folgende Fördermaßnahmen in Anspruch nehmen:

- Beratung nach der Unternehmensgründung im ersten Jahr nach der Aufnahme der selbstständigen Erwerbstätigkeit bzw. der Unternehmensgründung (20 Beratungstermine);

- eine Beihilfe von bis zu 3000 EUR für die Umsetzung des Geschäftsplans; und

- eine monatliche Zuwendung in Höhe des Mindestlohns (etwa 320 EUR) während der ersten sechs Monate nach der Unternehmensgründung.

Die NVA wählt die Anbieter der Beratungs- und Expertendienstleistungen durch ein öffentliches Vergabeverfahren aus.

Zwischen 2008 und 2014 nahmen insgesamt 1228 Arbeitslose an den anfänglichen Beratungen zur Vorbereitung eines Geschäftsplans teil (Tabelle 14.1). Etwa ein Viertel dieser Teilnehmer (377) unterzeichneten eineVereinbarung über ihren Übergang in die zweite Unterstützungsphase (d. h. zusätzliche Unternehmensberatung, Beihilfe und Überbrückungsgeld). Der beträchtliche Teilnehmerschwund zwischen der ersten und der zweiten Phase erklärt sich durch die große Anzahl von Menschen, die ihre Geschäftsidee nicht weiterverfolgen wollten, weil die Expertenevaluierung sie nicht als realisierbaren Plan für ein nachhaltiges Unternehmen unterstützte. 
Tabelle 14.1. Anzahl der Teilnehmer an der Maßnahme zur Aufnahme einer gewerblichen Tätigkeit oder selbstständigen Erwerbstätigkeit

\begin{tabular}{|c|c|c|c|c|c|c|c|c|c|c|c|c|c|c|c|}
\hline \multirow{3}{*}{$\begin{array}{l}\text { Beginn im } \\
\text { Jahr } \\
\\
2008\end{array}$} & \multicolumn{5}{|c|}{$\begin{array}{c}\text { Anzahl der Arbeitslosen, die } \\
\text { Beratungstermine } \\
\text { in Anspruch nahmen }\end{array}$} & \multicolumn{5}{|c|}{$\begin{array}{c}\text { Anzahl der Arbeitslosen, die eine } \\
\text { Vereinbarung über die Umsetzung eines } \\
\text { Geschäftsplans unterzeichneten (d. h. in } \\
\text { der zweiten Phase) }\end{array}$} & \multicolumn{5}{|c|}{$\begin{array}{c}\text { Anzahl der Unternehmer (vormals } \\
\text { Arbeitslose), } \\
\text { die zwei Jahre lang einen Geschäftsplan } \\
\text { umsetzten } \\
\text { (d. h. das Programm abschlossen) }\end{array}$} \\
\hline & \multirow{2}{*}{$\begin{array}{c}\text { Gesamt } \\
\mathbf{8 5}\end{array}$} & \multicolumn{2}{|c|}{ Männlich } & \multicolumn{2}{|c|}{ Weiblich } & \multirow{2}{*}{$\begin{array}{c}\text { Gesamt } \\
\mathbf{2 0}\end{array}$} & \multicolumn{2}{|c|}{ Männlich } & \multicolumn{2}{|c|}{ Weiblich } & \multirow{2}{*}{$\frac{\text { Gesamt }}{\mathbf{2 0}}$} & \multicolumn{2}{|c|}{ Männlich } & \multicolumn{2}{|c|}{ Weiblich } \\
\hline & & 33 & $38,8 \%$ & 52 & $61,2 \%$ & & 6 & $30,0 \%$ & 14 & $70,0 \%$ & & 6 & $30,0 \%$ & 14 & $70,0 \%$ \\
\hline 2009 & 256 & 101 & $39,5 \%$ & 155 & $60,5 \%$ & 83 & 33 & $39,8 \%$ & 50 & $60,2 \%$ & 75 & 28 & $37,3 \%$ & 47 & $62,7 \%$ \\
\hline 2010 & 139 & 50 & $36,0 \%$ & 89 & $64,0 \%$ & 37 & 12 & $32,4 \%$ & 25 & $67,6 \%$ & 35 & 12 & $34,3 \%$ & 23 & $65,7 \%$ \\
\hline 2011 & 234 & 67 & $28,6 \%$ & 167 & $71,4 \%$ & 56 & 13 & $23,2 \%$ & 43 & $76,8 \%$ & 54 & 12 & $22,2 \%$ & 42 & $77,8 \%$ \\
\hline 2012 & 265 & 59 & $22,3 \%$ & 206 & $77,7 \%$ & 85 & 23 & $27,1 \%$ & 62 & $72,9 \%$ & 83 & 23 & $27,7 \%$ & 60 & $72,3 \%$ \\
\hline 2013 & 140 & 22 & $15,7 \%$ & 118 & $84,3 \%$ & 53 & 11 & $20,8 \%$ & 42 & $79,2 \%$ & & & & & \\
\hline $\begin{array}{l}2014 \text { (die } \\
\text { ersten zehn } \\
\text { Monate) }\end{array}$ & 109 & 32 & $29,4 \%$ & 77 & $70,6 \%$ & 43 & 15 & $34,9 \%$ & 28 & $65,1 \%$ & & & & & \\
\hline Gesamt & 1228 & 364 & $29,6 \%$ & 864 & $70,4 \%$ & 377 & 113 & $30,0 \%$ & 264 & $70,0 \%$ & 267 & 81 & $30,3 \%$ & 186 & $69,7 \%$ \\
\hline
\end{tabular}

Quelle: NVA, 2014

Die Mehrheit der Programmteilnehmer seit 2008 waren Frauen (etwa 70\%). Die meisten Programmteilnehmer hatten eine höhere Bildungsstufe abgeschlossen (75,2\%). Diejenigen mit einer Berufsausbildung bzw. abgeschlossener Sekundarstufe machten 19,4\% bzw. 5,4\% der Teilnehmer aus. 67,4\% der Teilnehmer waren zwischen 30 und 49 Jahre alt (und stellten somit die Mehrheit dar), während Menschen über 50 Jahre 17,8\% und Menschen zwischen 20 und 29 Jahren 14,8\% derTeilnehmer ausmachten.

Ende 2014 hatten 267 Unternehmer das Zwei-Jahres-Programm abgeschlossen, und 129 Arbeitslose erhielten weiterhin Unterstützung. Langzeitarbeitslose machten fast ein Drittel dieser Teilnehmer aus (27,9\%). Weitere Schlüsselgruppen, die diese Unterstützung Ende 2014 in Anspruch nahmen, waren Menschen mit Behinderungen (6,2\%) und solche, die nach der Elternzeit wieder eine Erwerbstätigkeit aufnahmen (2,3\%). Junge Menschen (15- bis 24-Jährige) machten nur 4,7\% der Teilnehmer und damit einen geringeren Anteil als die Menschen aus, die kurz vor dem Ruhestand standen (5,4\%).

\section{Projektfinanzierung}

Im Jahr 2014 betrugen die Kosten des Programms 288300 EUR. Die Kosten pro Person für die verschiedenen Bestandteile werden in Tabelle 14.2 dargestellt.

Tabelle 14.2. Kosten der Maßnahme zur Aufnahme einer gewerblichen Tätigkeit oder selbstständigen Erwerbstätigkeit pro Person aufgeschlüsselt nach Bestandteilen, 2014

\begin{tabular}{|c|c|}
\hline Unterstützungsmaßnahme & Kosten pro Person \\
\hline Individuelle Beratungssitzung & 14,66 EUR \\
\hline Evaluierung von Geschäftsplänen & 32,71 EUR \\
\hline Überwachung von Geschäftsplänen & 30,59 EUR \\
\hline Einmalige Beihilfe für die Umsetzung des Geschäftsplans & 2845,74 EUR (maximal) \\
\hline Monatliche Zuwendung & 320 EUR (für sechs Monate) \\
\hline Maximale Finanzierung pro Teilnehmer & 5628,83 EUR \\
\hline
\end{tabular}

Quelle: NVA, 2014 


\section{Herausforderungen}

Die größte Herausforderung, mit der das Pilotprojekt konfrontiert war, war die Entwicklung eines Verfahrens zur Auswahl geeigneter Teilnehmer. Zwar führte die Berücksichtigung von Teilnehmerqualifikationen für das Programm zu guten Ergebnissen, doch die strengen Auswahlkriterien hinderten das Programm auch daran, größere Auswirkungen auf die Beschäftigung zu haben. Das Programm ist sehr klein, und eine größere Anzahl von Arbeitslosen könnte von der finanziellen Unterstützung und den Schulungen, die im Rahmen des Programms angeboten werden, profitieren. Eine Lockerung der Zugangsvoraussetzungen könnte das Programm für mehr Menschen öffnen und zur Schaffung von mehr Unternehmen und Arbeitsplätzen führen.

Eine zweite Herausforderung war die Unsicherheit, ob der Programmhaushalt aufgrund der kleinen Teilnehmerzahl während der Wirtschaftskrise aufrechterhalten werden würde. Obwohl das Programm als kleines Pilotprojekt konzipiert war, konnten die Beihilfen und Zuwendungen erwiesenermaßen mit Erfolg Unternehmensgründungen durch Arbeitslose fördern. Daher ist nun eine günstige Gelegenheit, eine Ausweitung des Programms in Betracht zu ziehen, um damit seine Auswirkungen auf die Beschäftigung zu vergrößern. Dazu muss die Inanspruchnahme steigen. Dies macht eine zielgerichtete Werbung, eine leichte Lockerung der Auswahlkriterien und die Verbesserung einiger Details der Bereitstellung der Unterstützung notwendig.

Und schließlich konkurriert der informelle Sektor zu einem gewissen Grad mit öffentlichen Unterstützungsprogrammen zur Unterstützung von Arbeitslosen. Laut wissenschaftlichen Forschungsarbeiten liegt die Quote informeller Erwerbstätigkeit recht hoch: Etwa 10,3\% der Arbeitslosen in Lettland arbeiteten 2013 ohne Vertrag (Putnins und Sauka, 2014). Dies deutet darauf hin, dass die informelle Erwerbstätigkeit in Lettland weiter verbreitet ist als in den Nachbarländern Litauen oder Estland. Daher muss eine öffentliche Unterstützung für Arbeitslose, und damit auch dieses Pilotprojekt, gegen die soziale Akzeptanz der informellen Erwerbstätigkeit und die Attraktivität einer informellen Tätigkeit ankommen.

\section{Auswirkungen}

Die NVA hat ein Überwachungssystem eingerichtet, um die von den Teilnehmern erzielten Ergebnisse zu verfolgen. Bei den Kohorten von 2012 bis Mitte 2014 wurden die folgenden Resultate erreicht:

- 5,6\% der durch die Teilnehmer gegründeten Unternehmen wurden von der NVA als sehr erfolgreich bewertet. Anders ausgedrückt waren die Umsätze dieser Unternehmen erheblich höher als ursprünglich geplant.

- Bei der Hälfte der gestarteten Unternehmen (53\%) konnten durch die Umsetzung des jeweiligen Geschäftsplans die prognostizierten Umsatzerwartungen während des ersten Jahres erfüllt werden, blieben jedoch im zweiten Jahr hinter den Umsatzerwartungen zurück. Allerdings haben sich viele dieser Unternehmen einen stabilen Kundenstamm erarbeitet und haben weiterhin das Potenzial, sich zu einem nachhaltigen Unternehmen zu entwickeln.

- Bei $17 \%$ der gestarteten Unternehmen blieben die Umsätze im zweiten Jahr erheblich hinter den Erwartungen zurück. In den meisten Fällen waren die schlechten Umsatzzahlen durch externe Faktoren zu erklären, z. B. Elternzeit oder Preiserhöhungen beim Anlagevermögen.

- Die Umsetzung des Geschäftsplans wurde bei 9,4\% der gegründeten Unternehmen als erfolglos betrachtet. In diesen Fällen waren die Teilnehmer nicht in der Lage, ihre Geschäftsidee wie geplant umzusetzen, und die Fachberater zogen in Zweifel, ob das Unternehmen nach Auslaufen der Unterstützung weiterbestehen könnte.

- $14 \%$ der Teilnehmer gründeten kein Unternehmen. 


\section{Voraussetzungen für die Übertragung}

Wenngleich das Projekt einen kleinen Umfang hat, konnte es eine Reihe von Arbeitslosen mit Erfolg bei der Entwicklung nachhaltiger Unternehmen unterstützen. Während seiner siebenjährigen Laufzeit sind einige zentrale Erfolgsfaktoren zu erkennen, die für eine erfolgreiche Übertragung des Projekts auf einen anderen Kontext von entscheidender Bedeutung wären.

1. Entwicklung klarer Auswahlkriterien. Für dieses Projekt wurden äußerst klare und strenge Auswahlkriterien festgelegt, die die Teilnehmer erfüllen müssen, um für eine Teilnahme infrage zu kommen, unter anderem Berufserfahrung und Qualifikationen. Diese Kriterien werden potenziellen Teilnehmern mitgeteilt und durch den Auswahlprozess bestätigt. Darüber hinaus durchlaufen die für die zweite Unterstützungsphase ausgewählten Unternehmer einen zweiten Screening-Prozess, bei dem das Potenzial ihres Geschäftsplans sowie die Wahrscheinlichkeit beurteilt werden, dass sich dieser zu einem nachhaltigen Unternehmen entwickeln lässt. Dieses klare Auswahlverfahren stellt sicher, dass die unterstützten Unternehmer auch mit der größten Wahrscheinlichkeit Erfolg haben.

2. Nutzung des Fachwissens des privaten Sektors im Auswahlverfahren. Die Auswahl der Teilnehmer erfolgt durch einen Ausschuss aus Branchenexperten und Geschäftsexperten, die die Geschäftsideen bewerten. Der Vorteil dieses Ansatzes gegenüber einer Bewertung durch die Programmleiter ist der, dass diejenigen, die am Markt tätig sind, besser in der Lage sind, zu verstehen, welche Projekte wohl gelingen werden.

3. Angebot einer stufenweisen Unterstützung. Die Unterstützung wird in dieser Maßnahme in zwei Phasen geleistet. Vor jeder Unterstützungsphase wird eine Evaluierung durchgeführt, und die zweite Phase ist viel intensiver als die erste. Dank diesem aus verschiedenen Phasen bestehenden Ansatz kann eine breitere Gruppe potenzieller Unternehmer bei der Entwicklung ihrer Idee von grundlegender Schulung und Unterstützung profitieren. Die zweite Phase stellt intensivere (und mit höheren Kosten verbundene) Unterstützung für eine kleine Zahl von Teilnehmern bereit, die während der ersten Phase Erfolg und Potenzial vorweisen konnten. Dieser Ansatz ermöglicht es politischen Entscheidungsträgern, vielen Unternehmern grundlegende Schulungen bereitzustellen und denjenigen eine intensivere Unterstützung zu bieten, die das größte Erfolgspotenzial haben.

4. Vielfältige Unterstützung. Diese Maßnahme bietet Unternehmensberatung, eine Beihilfe und eine Zuwendung. Die Bereitstellung eines Bündels ergänzender Unterstützungsmaßnahmen ermöglicht den Teilnehmern die Entwicklung unternehmerischer Fähigkeiten sowie ein gewisses Maß an Finanzierung, um ihre Idee umzusetzen und ihre Fähigkeiten in der Praxis anzuwenden. Die Zuwendung ist ein wichtiges Element des Programms, da sie den Unternehmern während eines befristeten Zeitraums, während sie ihr Unternehmen entwickeln, bei der Deckung ihrer Lebenshaltungskosten hilft.

\section{Literatur}

Putnins, T. und A. Sauka (2014), „Measuring the Shadow Economy Using Company Managers“, Journal of Comparative Economics, Bd. 43(2), Mai 2015, S. 471-490. http://dx.doi.org/10.1016/j.jce.2014.04.001

NVA (2014), NVA-Website, www.nva.gov.lv (Zugriff am 5. Januar 2015). 


\section{Förderungsfonds für das Unternehmertum, Litauen}

Diese Fallstudie beschreibt ein Beispiel für ein allgemeines Unternehmerprogramm, das vorrangig jungen Menschen, Älteren, Arbeitslosen und Menschen mit Behinderungen Zugang gewährt. Zu den verfügbaren Unterstützungsleistungen zählen Mikrokredite, Darlehensgarantien, Unternehmerschulungen und Unternehmensberatung. Die Ziele dieses Ansatzes sowie dessen Hintergrund werden beschrieben und die Funktionsweise des Programms erläutert. Zusätzlich werden Belege für die Auswirkungen des Programms angeführt. Ferner werden in der Beschreibung die Herausforderungen bei der Umsetzung des Programms und die Voraussetzungen für seine Übertragung auf einen anderen Kontext erörtert.

\section{Ziele}

Der Förderungsfonds für das Unternehmertum (Entrepreneurship Promotion Fund, EPF) wurde Ende 2009 eingerichtet und läuft noch heute. Der Fonds wurde initiiert durch das Ministerium für soziale Sicherheit und Arbeit, das Finanzministerium und INVEGA, einer Agentur, die für die Entwicklung kleiner und mittlerer Unternehmen in Litauen und die Erleichterung ihres Zugangs zu Finanzierung zuständig ist. INVEGA ist die Verwaltungsbehörde des EPF. Die Hauptziele des EPF sind folgende (INVEGA, 2015):

- die Erreichung der Bereitstellung kleiner Darlehen für selbstständig erwerbstätige Existenzgründer und KMU zu besseren Konditionen als den marktüblichen;

- die Verbesserung des Zugangs zu Startkapital für benachteiligte Zielgruppen, unter anderem für:

- Arbeitslose

- junge Unternehmer unter 29 Jahren

- ältere Unternehmer über 50 Jahre

- Menschen mit Behinderungen;

- die Erreichung der Zuweisung von mindestens 30\% der Schulungsplätze und 15\% der Darlehen an Menschen aus den prioritären Gruppen;

- der Anstieg der Zahl der selbstständig erwerbstätigen Existenzgründer und Unternehmensgründer in der litauischen Wirtschaft;

- die Erhöhung der Zahl der Unternehmer und selbstständig Erwerbstätigen in den Zielgruppen;

- die Ermutigung der Unternehmensgründer und derer, die eine selbstständige Erwerbstätigkeit aufnehmen möchten, Schulungen und Beratung in Anspruch zu nehmen; 
- die Erhöhung der Zahl der nachhaltigen neuen KMU und der neuen selbstständig Erwerbstätigen;

- die Verringerung der Arbeitslosigkeit und die anhaltende Aktivität von Menschen in der Geschäftswelt und auf dem Arbeitsmarkt; und

- die Leistung eines Beitrags zur Entwicklung einer tragfähigen Unternehmerkultur in Litauen.

\section{Hintergrund}

Ziel des Programms ist es, bei Existenzgründern Finanzierungs- und Wissenslücken zu schließen und damit auf die hohen Arbeitslosenquoten und die schwierigen wirtschaftlichen Bedingungen nach der Krise in allen Regionen Litauens zu reagieren. Indem es Arbeitslosen, jungen Menschen, Älteren und Menschen mit Behinderungen vorrangig Zugang zu den angebotenen Unterstützungsleistungen gewährt, trägt es dem Umstand Rechnung, dass die Menschen aus diesen Gruppen häufig vor größeren Problemen stehen.

Der EPF kombiniert Mikrokredite mit Schulungen für potenzielle Unternehmer, die eine Unternehmensgründung oder die Aufnahme einer selbstständigen Erwerbstätigkeit beabsichtigen. Die Mikrokreditprogramme sollen Unternehmen, die aufgrund ihrer geringen Größe, ihrer fehlenden Bonitätsgeschichte, ihres Risikos und nicht bankfähiger Projekte von den kommerziellen Kreditmärkten ausgeschlossen wären, einen Zugang zu kleinen Darlehen gewähren (Parker, 2002). Unternehmer und Inhaber kleiner Unternehmen müssen Darlehen zu Zinssätzen zurückzahlen, die ungefähr bei oder unter den marktüblichen Sätzen festgelegt sind. Mikrokreditprogramme können gute Verfahren darstellen, da sie, anders als Beihilfen, den Unternehmern eine Verpflichtung und kaufmännische Disziplin auferlegen und voraussetzen, dass die Leistungen überwacht werden (Hirsch und Wlaz, 2011). Existenzgründer können nicht die Bonitätsgeschichte vorweisen, die Finanzinstitute bei der Beantragung eines Kredits verlangen, da hierfür üblicherweise eine Marktpräsenz seit mindestens zwei Jahren erforderlich ist. Somit können Mikrokreditprogramme eine Finanzierungslücke schließen, indem sie bis zur Erreichung einer erfolgreichen Marktpräsenz Zugang zu Startkapital bieten (Ayayi, 2012). Die Bereitstellung ergänzender Schulungen und Beratung ist eine wirksame Vorgehensweise, um die Erfolgsaussichten und die Wahrscheinlichkeit der Rückzahlung durch die unterstützten Unternehmen zu erhöhen (OECD/EK, 2013).

\section{Aktivitäten}

In Zusammenarbeit mit der Litauischen Zentralen Kreditgenossenschaft (LZK), die die Funktion eines Finanzintermediärs bei der Beurteilung der Bewerbungen von Kandidaten übernimmt, bietet das EPF-Programm Mikrokreditfinanzierung und Schulungen für erfolgreiche Antragsteller. Die LZK unterhält Partnerschaften mit 15 Schulungsanbietern, um kostenfreie Schulungen und Unternehmensberatungsdienste für die Darlehensempfänger durchzuführen. Die LZK ist die Anlaufstelle für die Programmteilnehmer, wenn es um Beratung, Informationen und ergänzende Schulungen geht. Die Schlüsselelemente des EPF sind unter anderem:

- unabhängige finanzielle Bewertung der Anträge von Unternehmensgründern und selbstständig Erwerbstätigen, die seit weniger als einem Jahr tätig sind, durch die Kreditgenossenschaftsmitglieder der LZK;

- kurz- und langfristige Mikrokredite von bis zu 86000 LTL (etwa 25000 EUR) pro KMU. Somit fallen diese Kredite unter die De-minimis-Grenze (d. h. unter den Höchstbetrag von 200000 EUR über drei Jahre für ein Einzelunternehmen) (INVEGA, 2014);

- für die gewährten Darlehen ist keine Höchstlaufzeit festgelegt, allerdings müssen alle Darlehen von den Darlehensempfängern bis Ende Dezember 2018 zurückgezahlt werden;

- die meisten Darlehen werden für Investitionen zur Verfügung gestellt, aber auch Darlehen für Betriebsmittel sind möglich;

- die Zinssätze, die Existenzgründern für Mikrokredite berechnet werden, liegen unter den marktüblichen Sätzen;

- die Zinsmarge der Kreditgenossenschaft beträgt weniger als 3,49\%; 
- umfangreiche kostenlose Schulungen und Beratung für erfolgreiche Antragsteller, unter anderem zu den folgenden Aspekten der Führung eines kleinen Unternehmens:

- Geschäftsplanung (ein Pflichtbestandteil für alle Antragsteller);

- Finanzbuchhaltung einschließlich Steuerplanung;

- Rechtliche Aspekte des Unternehmensrechts;

- Betriebswirtschaft einschließlich Personalmanagement; sowie

- Marketing;

- Darlehensempfänger, die über keine vorherigen Geschäftserfahrungen verfügen, erhalten eine allgemeine Schulung zur Gründung eines Unternehmens.

Die Teilnahme an allen Schulungssitzungen und Beratungsterminen ist zwar nicht verpflichtend, doch erfreuen sich diese Berichten zufolge einer großen Beliebtheit unter den letztendlichen Empfängern (INVEGA, 2014). Neben diesem umfassenden Unterstützungspaket besteht ein Merkmal des Erfolgs des EPF darin, dass die Teilnehmer auch auf zusätzliche Finanzierungsinstrumente von INVEGA zurückgreifen können (INVEGA, 2014):

- Garantien von bis zu $80 \%$ des Darlehenswertes. Dieses Element ist von besonderer Bedeutung für Antragsteller, die nur begrenzte Sicherheiten haben, zum Beispiel die Mitglieder der zwei Zielgruppen junge Menschen und Arbeitslose.

- Zwei Kategorien von finanziellen Zuschüssen:

- Zinszuschüsse von bis zu 95\%, wodurch die Zinsrückzahlungen auf ein Minimum reduziert werden; und

- Zuschüsse zu Mitarbeitergehältern. Beschäftigen die Begünstigten Mitarbeiter, kommen sie unter Umständen für Teilzuschüsse zu Mitarbeitergehältern infrage.

Die Hauptbestandteile des EPF, seine Prozesse und seine Managementstruktur sind in den Abbildungen 15.1 und 15.2 dargestellt. Das Verfahren der Finanzintermediation, im Rahmen dessen Anträge von aufstrebenden Unternehmern und selbstständig Erwerbstätigen geprüft werden, wird von den Kreditgenossenschaftsmitgliedern der LZK durchgeführt. Erfolgreiche Antragsteller erhalten kleine kurzfristige Darlehen für Betriebsmittel oder langfristigere Darlehen für Kapitalanlagen. Alle Darlehensempfänger müssen Mitglieder oder Anteilseigner der Kreditgenossenschaft werden. Dazu ist eine Einlage bei der Kreditgenossenschaft in Höhe von mindestens 10\% der Darlehenssumme erforderlich. Diese wird dem Empfänger nach Rückzahlung des Darlehens erstattet.

\section{Abbildung 15.1. Umsetzung des Förderungsfonds für das Unternehmertum}

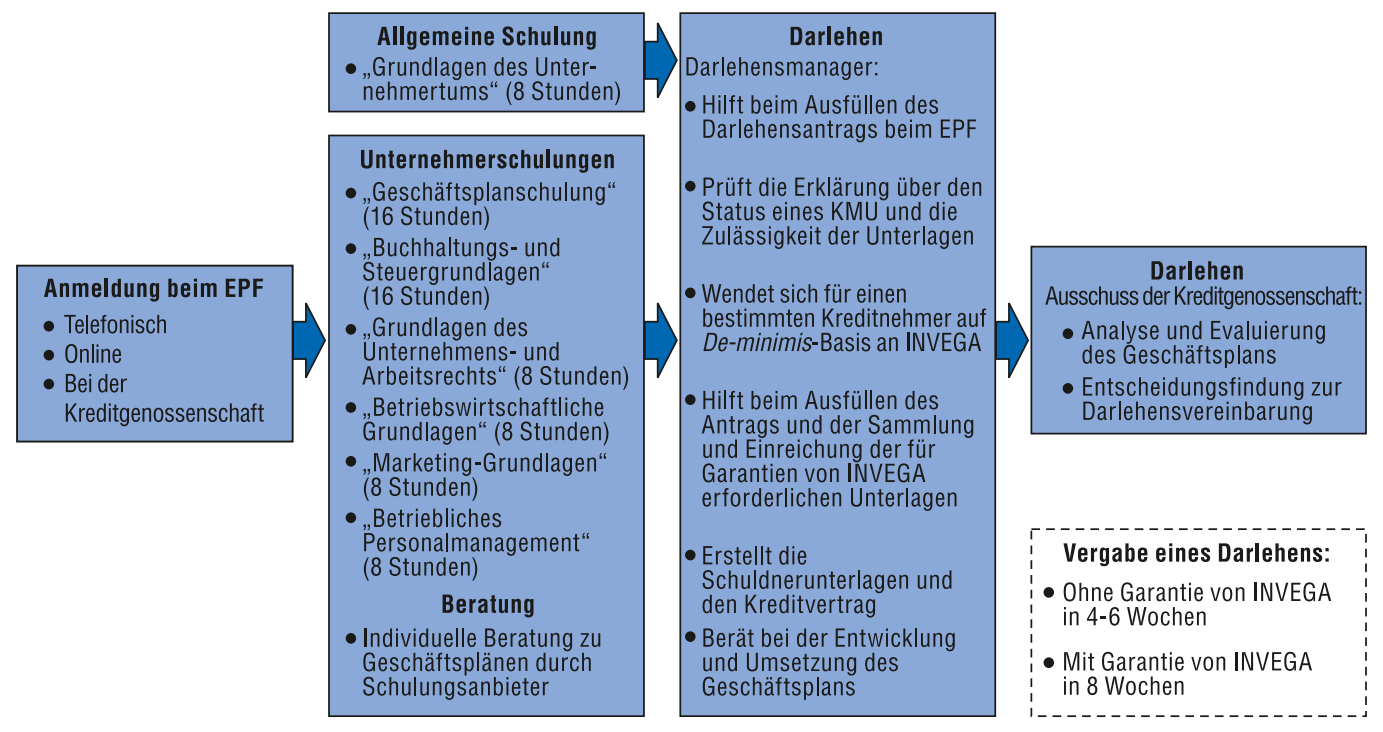




\section{Abbildung 15.2. Bestandteile des Programms „Förderungsfonds für das Unternehmertum“, 2009-2015}

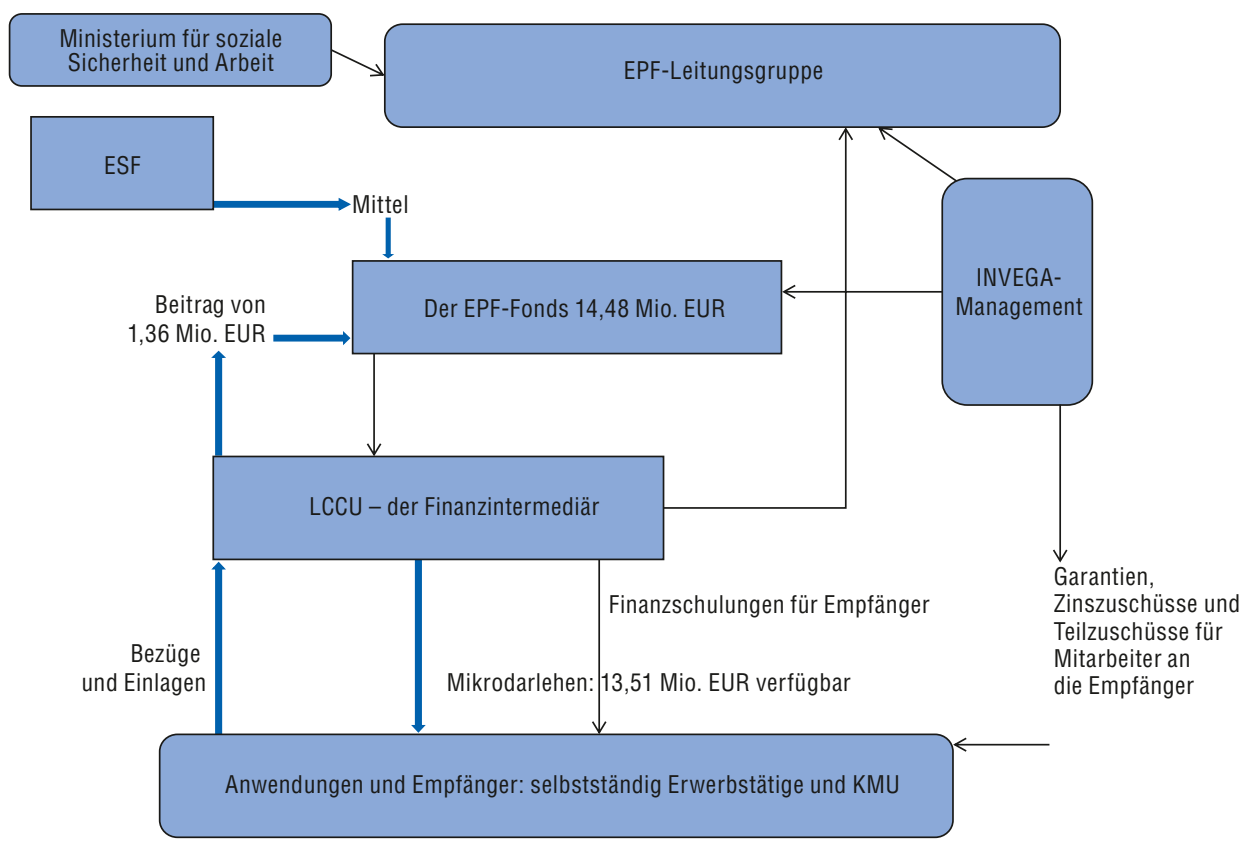

Jede Kreditgenossenschaft hat ihren eigenen Darlehensausschuss, und jeder Antragsteller wird einem Darlehensmanager zugewiesen, der eine Analyse der Geschäftsbedürfnisse und der persönlichen Fähigkeiten vornimmt. Vor Antragstellung wird eine Schulung zur Erstellungvon Geschäftsplänen angeboten. Der Antragsteller erhält Hilfestellung bei der Anfertigung des Darlehensantrags, des Geschäftsplanes und der Beantragung von Garantien von INVEGA. Die LZK bewertet den Antrag und den Geschäftsplan, und es wird eine Darlehensentscheidung getroffen. Ohne Garantien können Darlehen innerhalb von vier bis sechs Wochen gewährt werden; wird eine Garantie zur Absicherung des Darlehens benötigt, wird das Darlehen innerhalb von acht Wochen gewährt. (3) Derzeit werden 30\% der Vorschläge durch den Darlehensausschuss der Kreditgenossenschaft abgelehnt. $\left(^{4}\right)$

\section{Herausforderungen}

INVEGA hat eine Reihe von Herausforderungen bei der Einrichtung, der Verwaltung und der Durchführung des EPF festgestellt (INVEGA, 2014). Einige dieser Herausforderungen machten eine neue Ausrichtung und eine flexible Strategie notwendig, unter anderem:

- Die Bereitstellung einer finanziellen Unterstützung durch die Teilumwandlung von Darlehen in eine Beihilfe (zu 30\%) war eine Strategie, die zu Beginn des EPF im Jahr 2009 ursprünglich vorgesehen war (INVEGA, 2014). Allerdings verhindern Verordnungen der Europäischen Union die Umwandlung eines Teils des Darlehens in eine Beihilfe. Darum wurden andere Möglichkeiten der finanziellen Unterstützung untersucht. Das Wirtschaftsministerium einigte sich mit dem Ministerium für soziale Sicherheit und Arbeit auf die Einführung von Zinszuschüssen, die schließlich 2012 in den EPF aufgenommen wurden.

- Aufgrund der Komplexität derVerordnungen der Europäischen Union und der Finanzierungsbedingungen des Europäischen Fonds für regionale Entwicklung (EFRE) nahm die Einrichtung des EPF-Programms mehr Zeit in Anspruch als erwartet.

- Die Wirtschaftslage der jüngsten Zeit bedeutete, dass die Bedingungen für Unternehmensgründungen schwieriger waren als vorausgesehen und in der Folge die anfängliche Nachfrage nach Darlehen nicht so hoch war wie erwartet.

3 Diese Information ist einer Präsentation zu entnehmen, die INVEGA vor der OECD und internationalen Experten im November 2014 hielt.

4 Diese Information legte INVEGA der OECD im Februar 2015 per E-Mail vor. 
Diese Herausforderungen deuten auf die Notwendigkeit hin, die wirtschaftlichen Bedingungen regelmäßig zu überwachen und Programme flexibel zu gestalten, in diesem Fall etwa durch die Ermöglichung zusätzlicher Finanzierungsinstrumente. Der Kooperationsgrad zwischen den verschiedenen Einrichtungen spielte für den Erfolg des Programms eine wichtige Rolle, z. B. zwischen INVEGA und den Kreditgenossenschaftsmitgliedern der LZK.

Die Sicherung von Mitteln stellt ebenfalls eine Herausforderung für den EPF dar. Tabelle 15.1 veranschaulicht, dass die meisten Mittel, die zur Einrichtung des zentralen Fonds eingesetzt wurden, aus Geldern des Europäischen Sozialfonds (ESF) stammen. Von den 14,48 Millionen EUR wurden 2,32 Millionen EUR für Verwaltungsgebühren und die Schulungen der Empfänger bereitgestellt. Weiterhin hat das Konsortium der Kreditgenossenschaften, die LZK, 1,35 Millionen EUR in den zentralen Fonds eingezahlt, sodass dort 13,51 Millionen EUR zur Zuteilung an erfolgreiche Antragsteller zur Verfügung stehen. Der Beitrag der LZK (10\%) wurde in Dokumenten des öffentlichen Auftragswesens vereinbart und wird auf der Ebene der letztendlichen Empfänger ausgezahlt. Diese Auflage stellt sicher, dass neben dem zentralen Fonds zusätzlich private Ressourcen genutzt werden.

\section{Tabelle 15.1. Mittel für den zentralen Fonds des Förderungsfonds für das Unternehmertum, 2009-2015}

\begin{tabular}{lc}
\hline & Millionen EUR \\
\hline ESF-Gelder & 14,48 \\
Abzüglich Verwaltungsgebühren und Schulungsausgaben & 2,32 \\
Zuzüglich Beitrag der LZK & 1,35 \\
Insgesamt für Mikrokreditvergaben verfügbar & $\mathbf{1 3 , 5 1}$ \\
\hline
\end{tabular}

Quelle: INVEGA (2014b)

Die Mittel für die zusätzliche finanzielle Unterstützung in Form von Zuschüssen und Garantien im Rahmen des EPF-Programms stammen aus dem EFRE, dem ESF und nationalen Fonds. Diese tragen zu drei Fonds für Zinszuschüsse und Garantien (EFRE), Teilzuschüsse für Mitarbeiter (ESF) und finanzielle Garantien bei:

- Ein globaler Beihilfefonds über 16,2 Millionen EUR (EFRE) wurde für Zinszuschüsse an die Begünstigten des EPF eingerichtet. Bis zu 95\% der Darlehenszinsen sind bezuschusst.

- Ein globaler Beihilfefonds über 2,9 Millionen EUR (ESF) wurde für Teilzuschüsse für Mitarbeiter der Begünstigten eingerichtet. Der Wert der Lohnzuschüsse kann individuell bis zu 7240,50 EUR betragen, sofern der Begünstigte einer prioritären Gruppe angehört (Menschen unter 29 Jahren, über 50 Jahre, Arbeitslose oder Menschen mit Behinderungen).

- Ein Garantiefonds über 37,4 Millionen EUR (EFRE) wurde für Garantien eingerichtet. Garantien in Höhe von bis zu 80\% werden gewährt, falls die Antragsteller keine Sicherheiten haben. Diese Garantien können für Menschen aus prioritären Gruppen wie Arbeitslose und junge Menschen wichtig sein.

Grundsätzlich sollte sich ein erfolgreiches Mikrokreditprogramm durch die Zinsrückzahlungen der Begünstigten selbst tragen können. Bis Ende September 2014 lag derWert der mit Empfängern geschlossenen Darlehensverträge dank der Reinvestitionen um 24\% höher als die Gesamtmittel des EPF (INVEGA, 2014).

\section{Auswirkungen}

Der EPF wurde Ende 2009 eingerichtet; der erste Mikrokredit wurde im November 2010 vergeben. Die Zinszuschüsse wurden ab Januar 2012 und die Teilzuschüsse für Mitarbeiter ab September 2013 eingeführt. Das aktuell laufende Programm wurde bis 2018 verlängert.

Die wesentlichen Ergebnisse des Programms im Vergleich zu den Zielvorgaben für den Zeitraum von 2009 bis 2015 werden in Tabelle 15.2 aufgeführt (bis 31. September 2014). Die Daten zeigen auf, dass seit Ende 2010 mehr als 1000 Mikrokredite gewährt und insgesamt 1758 Arbeitsplätze geschaffen wurden. Die 
Überlebensrate der Begünstigten nach einem Jahr beträgt derzeit 97\% $\left(^{5}\right)$, was die Strenge widerspiegelt, mit der die Projekte der Antragsteller bewertet wurden.

\section{Tabelle 15.2. Auswirkungen des Programms „Förderungsfonds für das Unternehmertum“, 2010-2014}

\begin{tabular}{lcc}
\hline \multicolumn{1}{c}{ Indikator } & Ziel & $\begin{array}{c}\text { Ergebnisse bis zum } \\
31 . \text { September 2014 }\end{array}$ \\
\hline Teilnehmer an Schulungen & & 4205 \\
Teilnehmer, die Schulungen abgeschlossen haben & 5000 & 4117 \\
Teilnehmer an Schulungen aus prioritären Gruppen & 4500 & 2607 \\
Anzahl der gewährten Darlehen & 1500 & 1017 \\
Anzahl der an prioritäre Gruppen vergebenen Darlehen & 1200 & 479 \\
Anzahl der neu geschaffenen Arbeitsplätze & 180 & 1758 \\
\hline
\end{tabular}

Quelle: INVEGA (2014a)

Dem Programm gelang es außerdem, Menschen aus seinen prioritären Gruppen anzuwerben, $d . h$. Menschen unter 29 Jahren, Menschen über 50 Jahre, Menschen mit Behinderungen und Arbeitslose. Diese Gruppen erhielten $47 \%$ der gewährten Darlehen. Erreicht werden konnte dies durch die Nutzung der 154 Verkaufspunkte (d. h. durch das LZK-Netzwerk), mit denen die Zugänglichkeit des EPF insbesondere im ländlichen Raum gewährleistet wurde (INVEGA, 2014).

Darüber hinaus konnten die gegründeten Unternehmen, die durch den EPF gefördert wurden, einfacher kommerzielle Bankdarlehen auf den allgemeinen Kreditmärkten beantragen und nutzen, da sie durch den vom EPF gewährten Mikrokredit eine Bonitätsgeschichte aufgebaut hatten.

Ferner schienen die Kreditgenossenschaftsmitglieder der LZK einer Zusammenarbeit mit Kleinunternehmern und selbstständig Erwerbstätigen dank der Erfahrung mit dem EPF positiver gegenüberzustehen (INVEGA, 2014).

\section{Voraussetzungen für die Übertragung}

Mikrokreditprogramme haben das Potenzial, Gruppen mit niedrigem Einkommen und benachteiligten Gruppen bei der Unternehmensgründung zu helfen. Eine Reihe von Kriterien und Bedingungen muss erfüllt sein, damit ein ähnliches Programm wie der litauische EPF Erfolg hat:

1. Risikostreuung. Die Auswahl der Kandidaten für Darlehen muss ein zuverlässiger und angesehener Intermediär übernehmen, der das notwendige Finanzfachwissen besitzt, um eine Risikobewertung der Kandidaten vorzunehmen. Im Fall von Litauen war das Konsortium der Kreditgenossenschaften, die LZK, eine einzigartige und zuverlässige Stelle, die zur Ausübung dieser Funktion fähig war.

2. Nutzung von Glaubwürdigkeit. Der zentrale oder Holding-Fonds sollte unabhängig sein und durch eine renommierte und angesehene Verwaltungsstelle verwaltet werden. Im Fall von Litauen übernahm INVEGA, eine in der Wirtschaftsgemeinschaft hoch angesehene Einrichtung, diese Verwaltungsfunktion.

3. Ermöglichung des Zugangs zu nicht finanzieller Unterstützung. Mikrokreditprogramme allein bieten wahrscheinlich nicht ausreichend Unterstützung, um erfolgreiche Unternehmensgründungen zu garantieren. Optimalerweise werden sie durch zusätzliche Schulungen oder Mentoring für die Begünstigten ergänzt. Wenn diese Unterstützungsangebote kostenlos oder gegen nominale Gebühren bereitgestelltwerden sollen, sind zusätzliche Mittelerforderlich.Allerdings kann ein Mikrokreditprogramm zusammen mit Mentoring eine größere Inanspruchnahme solcher Beratungen und Schulungen durch im Unternehmertum benachteiligte Gruppen fördern.

5 Diese Information legte INVEGA der OECD im Februar 2015 per E-Mail vor. 
4. Sicherstellung der Zugänglichkeit. Es ist wichtig, sicherzustellen, dass das Programm für prioritäre oder Zielgruppen zugänglich ist. Dies kann bedeuten, dass eine Sensibilisierungskampagne durchgeführt werden muss, damit das Programm gut angenommen wird. In Litauen kümmerten sich Partner und Einrichtungen um das Marketing und die Öffentlichkeitsarbeit.

5. Einräumung von Flexibilität bei der Umsetzung. In den Vorkehrungen ist möglicherweise Flexibilität vonnöten, um strategischen Änderungen, der Erreichung der Zielgruppen und Veränderungen der wirtschaftlichen Bedingungen Rechnung zu tragen.

\section{Literatur}

Ayayi, A.G. (2012), „Micro-credit and Micro-equity: the David and Goliath of Micro-enterprise Funding“, Economic Papers, Bd. 31, Nr. 2, S. 244-254.

Bank von Litauen (2014), „Macroeconomic Forecast, December 2014“, Vilnius, Litauen, Bank von Litauen, verfügbar unter: www.lb.lt/macroeconomic_forecast (Zugriff am 10. Februar 2015).

EMN (2011), Microfinance: An economic integration tool for young people in Europe, Paper Nr. 9, Dezember, Brüssel, Europäisches Mikrofinanzierungsnetz.

Grameen Bank (2015), monatliches Update der Grameen Bank, verfügbar unter: www.grameen.com (Zugriff am 12. Februar 2015).

Hirsch, J. und U. Wlaz (2011), „Financing Decisions along a Firm's Life-cycle: debt as a commitment device“, European Financial Management, Bd. 17, Nr. 5, S. 898-927.

INVEGA (2015), „Purposes of Activities“, Vilnius, Litauen, INVEGA, verfügbar unter: www.invega.lt/en/invega/ about-invega.htm (Zugriff am 10. Februar 2015).

INVEGA (2014), Aus Materialien, die für eine Fallstudie für ,fi-compass“ zum „Förderungsfonds für das Unternehmertum, Litauen“ erstellt wurden, Brüssel, Europäische Investitionsbank, Dezember (siehe auch die Website von fi-compass www.fi-compass.eu).

OECD/Europäische Kommission (2013), Die fehlenden Unternehmer: Politiken für ein integrationsförderndes Unternehmertum in Europa, Paris, OECD Publishing. http://dx.doi.org/10.1787/9789264188419-de

Parker, S. (2002), „Do Banks Ration Credit to New Enterprises? And should Governments Intervene?“ Scottish Journal of Political Economy, Bd. 49, S. 162-195. 


\title{
Sozialhilfe für selbstständig Erwerbstätige (BBZ), Niederlande
}

\begin{abstract}
Diese Fallstudie stellt ein integriertes Unterstützungsprogramm für Arbeitslose vor, die eine Unternehmensgründung anstreben. Das Programm bietet Schulungen, Coaching und Mentoring, Beihilfen, Unternehmensberatung und eine Überbrückungshilfe. In der Beschreibung werden die Ziele, der Hintergrund sowie die Aktivitäten und Auswirkungen des Programms behandelt. Außerdem werden die bei der Bereitstellung dieser Unterstützung sichtbar werdenden Herausforderungen und die Voraussetzungen für die Übertragung dieser Art von Programm auf einen anderen Kontext erörtert.
\end{abstract}

Ziele

Bijstandverlening aan zelfstandigen (BBZ) („Sozialhilfe für selbstständig Erwerbstätige“) wurde ins Leben gerufen, um Sozialhilfeempfängern bei der Gründung eines kleinen Unternehmens und der Aufnahme einer selbstständigen Erwerbstätigkeit zu helfen. Es handelt sich um ein Instrument, durch das kommunale Regierungen neue Unternehmer durch die Bereitstellung von Schulungen, Coaching und finanzieller Unterstützung unterstützen. Das übergeordnete Ziel des Projekts besteht darin, die Beschäftigung zu steigern und die Anzahl der Menschen zu verringern, die Arbeitslosenleistungen beziehen.

\section{Hintergrund}

BBZ wurde 1996 von der nationalen Regierung als potenzielle Lösung zur Bekämpfung der hohen Arbeitslosigkeit eingerichtet und läuft noch heute. BBZ wird von den Stadtverwaltungen verwaltet, die für die Bereitstellung der Unterstützung private Unternehmensförderungsdienstleister, Unternehmensberater und Betreuer hinzuziehen. Die Stadtverwaltungen setzen dieses Instrument gelegentlich ein, um bestimmte Zielgruppen unter den Langzeitarbeitslosen zu erreichen. Dies geschieht häufig auf lokaler Ebene auf Projektbasis, wenn Dritte sich bereit erklären, solche Initiativen mitzufinanzieren (d. h. häufig im Rahmen von Projekten des Europäischen Sozialfonds).

\section{Aktivitäten}

BBZ bietet eine umfassende Palette an Interventionen, die alle darauf ausgerichtet sind, Arbeitslose oder andere Sozialhilfeempfänger bei der Gründung eines eigenen Unternehmens zu unterstützen. Der Prozess besteht aus acht einzelnen Schritten (Abbildung 16.1). Die Teilnehmer müssen nicht unbedingt alle der Dienste in Anspruch nehmen, doch die große Mehrheit tut dies. Teilnehmer benötigen etwa neun Monate, um das Aufnahmeverfahren zu durchlaufen und ihr Unternehmen zu gründen. 
Abbildung 16.1. Der BBZ-Prozess

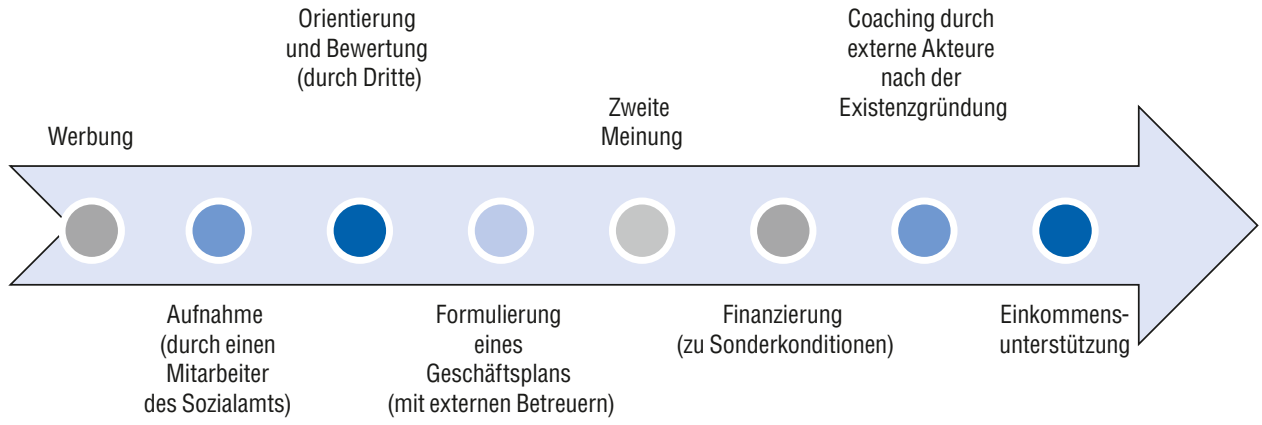

Der erste Schritt des Prozesses ist Werbung. Über die Stadtverwaltungen (d. h. das Sozialamt) erhalten die Zielgruppen allgemeine Informationen zu dem Programm. Die nationale Regierung stellt Websites, Prospekte und Broschüren bereit, durch die das Programm, seine Verfahren und seine Bedingungen erläutert werden. Die Stadtverwaltungen können flexibel entscheiden, wie die Informationen an potenzielle Klienten verbreitet werden. Häufig werden Partnerschaften mit halbstaatlichen Einrichtungen (z. B. Handelskammern, Unternehmensagenturen) genutzt, um die Kontaktarbeit auszuweiten.

Das Auswahlverfahren wird auf kommunaler Ebene von den Sozialämtern durchgeführt. Die Kommunalbeamten führen anhand eines systematisierten und dokumentierten Verfahrens, das den potenziellen Klienten in einem Leitfaden erläutert wird, Gespräche durch (Divosa, 2010). Ziel dieser Gespräche ist eine Einschätzung des Potenzials für eine Unternehmensgründung und eine selbstständige Erwerbstätigkeit. Der Sozialamtsmitarbeiter kann den Klienten an spezialisierte (private und halbstaatliche) Agenturen verweisen, bei denen er Schulungen und Beratung erhält. In einigen Stadtverwaltungen werden die Sozialamtsmitarbeiter speziell darin geschult, wie diese Gespräche durchzuführen sind, jedoch wird dies nicht landesweit konsequent vorgenommen.

DernächsteSchrittfür dieTeilnehmerbesteht aus einer OrientierungundUnternehmerschulungen. Die Agenturen, die die Schulungen und Unternehmensberatungsdienste bereitstellen, nehmen eine ausführlichere Analyse des unternehmerischen Potenzials der Klienten vor, bei der sie sich auf ihre Erfahrungen, Fähigkeiten und Bedürfnisse konzentrieren. Für diese Analysen entwerfen die Agenturen eigene Prozesse. Durchschnittlich dauert das Orientierungsprogramm zwölf Stunden verteilt auf vier bis sechs Tage. Der Orientierungsprozess kann eine kurze einführende Unternehmerschulung beinhalten. Ein weiteres denkbares Ergebnis ist, dass der Klient merkt, dass eine selbstständige Erwerbstätigkeit keine realisierbare Option für ihn darstellt. In diesem Fall kann er aus dem Programm aussteigen. Nach dem Orientierungsprozess geben die Agenturen gegenüber dem Sozialamtsmitarbeiter eine Empfehlung darüber ab, ob der Teilnehmer das Programm fortsetzen sollte.

Die Klienten, die das Unterstützungsprogramm fortsetzen, gehen in die Phase über, in der ein Geschäftsplan formuliert wird. Dort erhalten sie individuelle Unterstützung bei der Entwicklung von Geschäfts- und Finanzplänen. Diese Unterstützung wird durch privatwirtschaftliche Einrichtungen bereitgestellt, die von den Stadtverwaltungen beauftragt werden. In dieser Phase kommen die Klienten für einen kleinen vorbereitenden Kredit über bis zu 2000 EUR infrage, um spezielle Aktivitäten wie Marktforschung oder weitere technische oder Managerschulungen zu finanzieren. Dieser Kredit wird in die Gesamtdarlehenssumme eingerechnet, sobald die Geschäfts- und Finanzpläne genehmigt werden.

Wenn ein potenziell realisierbarer Geschäftsplan abgeschlossen ist, kann ihn die Stadtverwaltung einem Dritten vorlegen, um eine zweite Meinung einzuholen. Dies ist obligatorisch für beantragte Darlehenssummen von über 10000 EUR. Bei Darlehen unter 10000 EUR holen die Stadtverwaltungen in der Regel auch eine zweite Meinung bei Dritten ein, sind aber nicht dazu verpflichtet. Für diese Konsultation wendet man sich fast immer an die private nationale KMU-Agentur IMK Advies. 
Fällt die eingeholte zweite Meinung positiv aus, kommen die Klienten für eine Finanzierung durch die Stadtverwaltung infrage. Bei dem verwendeten Instrument handelt es sich um ein nachrangiges Darlehen von bis zu 35130 EUR zu 8\% Zinsen p. a. seit dem 1. Juli 2009. Davor lag der Zinssatz bei $5 \%$. Inhaber bestehender Kleinunternehmen, die vor dem Konkurs stehen, können ein Darlehen von bis zu 189000 EUR erhalten. In den meisten Fällen erhalten die Teilnehmer Darlehen von weniger als 20000 EUR. Die nachrangigen Darlehen werden zum Teil durch das Betriebsvermögen besichert, und für die Rückzahlung wird üblicherweise eine Laufzeit von fünf Jahren festgelegt. Voraussetzung für die Gewährung dieses Darlehens ist, dass der Klient keine Finanzierung von sonstigen formellen Finanzinstituten oder Banken erhalten konnte. Die Darlehen werden durch die Stadtverwaltungen verwaltet und die Buchhaltung erfolgt in bar, nicht auf Transaktionsbasis. Die nationale Regierung erstattet den Stadtverwaltungen (über das Ministerium für Soziales und Beschäftigung) jährlich bis zu $75 \%$ der ausstehenden Darlehenssummen.

Nach der Unternehmensgründung kommen Klienten für eine Einkommensunterstützung infrage. Die Stadtverwaltungen schließen in der Regel Sondervereinbarungen über die fortgesetzte Zahlung ihrer Sozialhilfeleistungen für ein Jahr ab. Diese können auf bis zu drei Jahre verlängert werden. Diese Überbrückungshilfe wird von den Nettoerträgen abgezogen, die das Unternehmen erwirtschaftet. In ähnlicher Weise können Teilnehmer, die Arbeitslosenleistungen beziehen, für denselben Zeitraum unter Umständen 54,9\% ihrer Arbeitslosenleistung erhalten.

Klienten, deren Geschäftspläne genehmigt wurden - und die somit entweder ein Darlehen oder weiterhin eine Einkommensunterstützung erhalten -, können außerdem während der Unternehmensgründung und bis $\mathrm{zu}$ zwölf Monate lang danach Coaching und Unternehmensberatungsdienste in Anspruch nehmen. Diese individuelle Unterstützung wird durch den Unternehmensberater gesteuert und beinhaltet in der Regel die Themenbereiche Kostenrechnung und Preisbildung, die Erfüllung von Verwaltungsauflagen, Umsatz- und Einkommensteuer, Liquiditätsplanung sowie Vertrieb und Marketing. Sie wird von Fachleuten bereitgestellt, die entweder für private Wirtschaftsförderungsdienste oder halbstaatliche Einrichtungen tätig sind, die durch die Stadtverwaltungen beauftragt werden. Die Stadtverwaltungen zahlen für diese Dienstleistungen eine im Voraus festgelegte Pauschale.

\section{Projektpartner}

Das nationale Ministerium für Soziales und Beschäftigung überwacht die Gesamtumsetzung der politischen Maßnahme, sorgt für die Finanzierung und nimmt regelmäßige Evaluierungen und Qualitätskontrollen vor.

Die kommunalen Abteilungen für Soziales und Beschäftigung sind in erster Linie für die Umsetzung des Programms zuständig. Ihre Mitarbeiter spielen bei der Umsetzung eine Schlüsselrolle. Sie identifizieren potenzielle Klienten und genehmigen diejenigen unter ihnen, die in das Programm aufgenommen werden sollen. Die Finanzierung, einschließlich der Vergabe und Verwaltung der Darlehen, erfolgt direkt durch die Stadtverwaltungen. In einigen wenigen Fällen arbeiten die Stadtverwaltungen mit Kommunalbanken zusammen, die Unterstützung bei der Verwaltung der Darlehen leisten.

Privatwirtschaftliche Wirtschaftsförderungsdienste und halbstaatliche Einrichtungen werden durch die Stadtverwaltungen mit der Bereitstellung der Dienstleistungen beauftragt. Die Stadtverwaltungen entscheiden nach eigenem Ermessen, wie sie die Dienstleister für Schulungen und Unternehmensberatung beauftragen. Viele Stadtverwaltungen nutzen Festaufträge (durch beschränkte und auch manchmal offene Ausschreibungsverfahren) mit einem oder zwei Dienstleistern für einen festgelegten Zeitraum.

Externe privatwirtschaftliche Wirtschaftsförderungsdienste (z. B. IMK Advies) und halbstaatliche Akteure (z. B. Handelskammern) werden mit der Validierung potenzieller Klienten und ihrer Geschäftspläne beauftragt. 


\section{Projektentwicklung}

In den letzten Jahren wurde eine Reihe von großen Veränderungen vorgenommen. Erstens wurde das Programm immer weiter dezentralisiert, und die Stadtverwaltungen erhielten mehr Kontrolle über die Programmumsetzung. Dies hat dazu geführt, dass mehr Partner an dem Programm mitwirken, sodass es zu einer verstärkten Abstimmung zwischen den Stadtverwaltungen kam, um ein bestimmtes Maß an Skaleneffekten zu erreichen und die lokal verfügbaren Kenntnisse und Erfahrungen besser auszuschöpfen.

Im Zeitraum 2009-2011 wurde ein Pilotprojekt durchgeführt, um eine Unterstützung für Arbeitslose zu testen, im Rahmen derer ein Garantieprogramm zur Anregung der Kreditvergabe an Arbeitslose mit Schulungen und Unternehmensberatung kombiniert wurde. Diesem Pilotprojekt gelang es nicht, Banken zu ermutigen, Kredite an diese Klientengruppe zu vergeben.

Und schließlich werden die für Schulungen und Unterstützungsdienste gezahlten Beträge regelmäßig an die Marktentwicklungen und die veränderten Staatshaushalte angepasst.

Außerdem wird das Programm eingesetzt, um etablierte ältere Unternehmer (Unternehmer über 55 Jahre, die seit mindestens zehn Jahren keine Sozialleistungen bezogen haben) zu unterstützen, die vorübergehende Schwierigkeiten haben, und um etablierten Unternehmern mit nicht tragfähigen Unternehmen bei der Liquidation ihres Geschäfts zu helfen.

\section{Projektfinanzierung}

Im Jahr 2012 betrugen die Gesamtkosten des BBZ 40,4 Millionen EUR (Tabelle 16.1). Fast drei Viertel der Mittel wurden für die Einkommensunterstützung aufgewendet. 21\% wurden für Umsetzungskosten (d. h. Kosten für Verwaltungs- und Wirtschaftsförderungsdienste) und 7\% für die Finanzierung der Darlehenstransaktionen verwendet.

Tabelle 16.1. Finanzierung von BBZ durch die nationale Regierung, 2012

\begin{tabular}{lcc}
\hline \multicolumn{1}{c}{ Kategorie } & Betrag in Euro & $\begin{array}{c}\% \text { des } \\
\text { Gesamtbetrags }\end{array}$ \\
\hline $\begin{array}{l}\text { BBZ-Einkommensunterstützung für Langzeitarbeitslose, die ein Kleinunternehmen } \\
\text { gründen bzw. eine selbstständige Erwerbstätigkeit aufnehmen }\end{array}$ & 29000000 EUR \\
& \\
BBZ-Finanzierung - Nettoauszahlung nach Abzug der Rückzahlungen im selben Jahr \\
von Darlehen aus früheren Jahren
\end{tabular}

Die Mittel für BBZ stammen aus dem Haushalt des Ministeriums für Soziales und Beschäftigung. Über die Jahre haben verschiedene Stadtverwaltungen spezielle Projekte für besondere Zielgruppen gestartet (z. B. junge Menschen, Frauen, Zuwanderer), die durch die BBZ-Mittel und den ESF oder andere Fonds der Europäischen Union kofinanziert wurden. Diese kofinanzierten Projekte waren fast alle lokale Projekte. BBZ erhält nicht laufend Mittel aus Fonds der Europäischen Union.

Eine Aufschlüsselung der Kosten für die Unterstützungsdienste ergab, dass die Gesamtkosten für die Bereitstellung der Wirtschaftsförderungsdienste (d. h. Coaching und Beratung) 9650 EUR betrugen (Tabelle 16.2). 


\section{Tabelle 16.2. Kosten der einzelnen Wirtschaftsförderungsdienste des BBZ und Klientenanzahl pro Jahr, 2012}

\begin{tabular}{lcc}
\hline \multicolumn{1}{c}{ Art des Wirtschaftsförderungsdienstes } & $\begin{array}{c}\text { Kosten pro Wirtschaftsförderungsdienst } \\
\text { und Klient }\end{array}$ & $\begin{array}{c}\text { Relative Kosten } \\
\text { (im Durchschnitt) }\end{array}$ \\
\hline Orientierung vor Existenzgründung, Coaching & 3050 EUR & $31,61 \%$ \\
Coaching für die Formulierung eines Geschäftsplans & 1800 EUR & $18,65 \%$ \\
Zweite Meinung & 2000 EUR & $20,73 \%$ \\
Coaching nach der Existenzgründung & 2800 EUR & $29,02 \%$ \\
Gesamtkosten für Wirtschaftsförderungsdienste & $\mathbf{9 6 5 0 ~ E U R ~}$ & 1200 \\
\hline
\end{tabular}

\section{Herausforderungen}

Die über die Jahre aufgetretenen Herausforderungen werden im Folgenden erörtert. Erstens gestaltet sich die Festlegung eines angemessenen Preis/Kosten-Verhältnisses bei den Wirtschaftsförderungsdiensten als schwierig. Die festgelegten Preise, die für diese Dienstleistungen gezahlt werden können, werden regelmäßig angepasst, aber die Änderungen sind eher marginal. Sie machen derzeit fast $40 \%$ der durchschnittlichen Darlehenssummen aus, was im Vergleich zu anderen Programmen hoch ausfällt; diese Kosten betragen in der Regel 25\% der Investitionen. Die privaten Dienstleister bringen in regelmäßigen Abständen vor, die Zahlungen seien zu gering, und setzen die Behörden unter Druck, die Zahlungen zu erhöhen, ohne dass sich die erbrachten Dienstleistungen erheblich ändern (Bobbezoo, 2010).

Zudem ist es schwierig, Wirtschaftsförderungsdienste $\mathrm{zu}$ finden und Beziehungen mit ihnen aufzubauen. Während der Anfangsjahre des Programms (d. h. Ende der 1990er-Jahre) waren mehr als 20 Dienstleister an dem Programm beteiligt. Viele wurden beauftragt, lokale oder regionale Projekte, die durch BBZ und den ESF kofinanziert wurden, zusammen mit den Stadtverwaltungen umzusetzen (z. B. Emploi). Allerdings gelang es vielen im Laufe der Zeit nicht, sich Aufträge von anderen Parteien zu sichern, und als die Projektmittel ausliefen, entschlossen sich viele von ihnen, die Tätigkeit aufzugeben. In der Folge ist die Zahl der Dienstleister stark zurückgegangen. Dies wird sich wahrscheinlich in der Zukunft negativ auf die Qualität der Dienstleistungen auswirken.

Die Verwaltung und Buchhaltung des Programms erfolgte hauptsächlich auf der Grundlage von Barmitteln, was für die staatliche Haushaltsplanung üblich ist. Den Stadtverwaltungen wurden die ausstehenden Nettobeträge jährlich erstattet (d. h. gewährte Darlehen abzüglich der Rückzahlungen). Das macht es recht schwierig und umständlich, die Leistung einzelner Klienten zu verfolgen. Derzeit werden die Stadtverwaltungen angeregt, ein genaueres Verwaltungssystem einzurichten.

Zudem hat das Programm einige Schwierigkeiten bei der Anpassung an die Bedürfnisse der Unternehmer. Immer häufiger kombinieren Unternehmer ihre selbstständige Erwerbstätigkeit mit einer abhängigen Erwerbstätigkeit und anderen Tätigkeiten. Dies hat Folgen für die Arbeitslosen, denn eine in Teilzeit ausgeübte selbstständige Erwerbstätigkeit kann zu einer Kürzung ihrer Leistungen führen, selbst wenn ihr Unternehmen keine oder geringe Erträge erwirtschaftet.

Die gesellschaftliche Vielfalt in den Niederlanden nimmt zu, weshalb öffentliche Arbeitsverwaltungen und Wirtschaftsförderungsdienste dazu gezwungen sind, ihre Dienstleistungen und ihre Kontaktarbeit anzupassen. Beide müssen diese Herausforderungen bewältigen, indem sie ihre Geschäftsmodelle neu definieren und ihren Mitarbeitern neue Schulungen zur Verfügung stellen.

Sehr wenige Mitarbeiter der Stadtverwaltungen haben einen ökonomischen Hintergrund oder Erfahrungen mit der Geschäftswelt. Daher ist es für sie schwierig, die unternehmerischen Kompetenzen von Klienten angemessen zu bewerten oder ihre Geschäftsideen zu beurteilen. Auch tendieren sie dazu, die Aufsicht und Überwachung der Leistung der gegründeten Unternehmen zu übergehen. Es wurden noch keine systematischen Mitarbeiterschulungen in diesen Bereichen eingeführt. 


\section{Auswirkungen}

Pro Jahr bekunden rund 5000 Menschen Interesse an dem Programm, und etwa die Hälfte nimmt am Aufnahmeverfahren teil (SEO, 2008). Während des Aufnahmeverfahrens steigen etwa $20 \%$ wieder aus, und zwischen 1800 und 2400 Menschen pro Jahr beginnen das Programm. Die Anzahl der Unternehmensgründungen reicht von 900 bis 1200 im Jahr. Die durchschnittliche Darlehenssumme liegt zwischen 17000 EUR und 19000 EUR und ist vergleichbar mit dem Durchschnitt der Darlehen, die Qredits, die führende Mikrofinanzierungsinstitution in den Niederlanden, vergibt.

Eine kürzlich durchgeführte Evaluierung (Ecorys, 2011) zeigt, dass während der vergangenen fünf Jahre $72 \%$ der Menschen, die im Rahmen von BBZ unterstützt wurden, nach drei Jahren immer noch selbstständig erwerbstätig (oder angestellt) waren. Bei der Evaluierung wurde eine KostenNutzen-Analyse des Programms vorgenommen. Dabei wurde festgestellt, dass der Nettonutzen für die Gesellschaft pro Unternehmensgründung 18900 EUR betrug (Tabelle 16.3).

Tabelle 16.3. Schätzung der Kosten und Nutzen von BBZ pro Existenzgründer

\begin{tabular}{|c|c|c|c|}
\hline \multicolumn{4}{|c|}{ Kosten/entgangene Einnahmen der Zentralregierung } \\
\hline & $\begin{array}{l}\text { \% der Existenzgründer/ } \\
\text { Korrekturfaktor }\end{array}$ & Durchschnittliche Kosten pro Person & $\begin{array}{l}\text { Durchschnittliche Kosten pro } \\
\text { Existenzgründer }\end{array}$ \\
\hline $\begin{array}{l}\text { Einkommensunterstützung während der Schulungen/der } \\
\text { Vorbereitung }\end{array}$ & 160 & 8800 EUR & 14080 EUR \\
\hline Betreuer vor der Existenzgründung & 160 & 3050 EUR & 4880 EUR \\
\hline Vorbereitender Kredit & 15 & 2000 EUR & 300 EUR \\
\hline Vorbereitung/Coaching für die Existenzgründung & 160 & 1800 EUR & 2880 EUR \\
\hline Zweite Meinung & 200 & 2000 EUR & 4000 EUR \\
\hline $\begin{array}{l}\text { Fortgesetzte Zahlung der Einkommensunterstützung/der } \\
\text { Sozialleistungen }\end{array}$ & 100 & 56000 EUR & 56000 EUR \\
\hline Unternehmensdarlehen & 54 & 18000 EUR & 9720 EUR \\
\hline Coaching nach der Existenzgründung & 45 & 1200 EUR & 540 EUR \\
\hline Umsetzungskosten während der Existenzgründung & 100 & 2800 EUR & 2800 EUR \\
\hline $\begin{array}{l}\text { Abzüglich Einkommensteuern bei abhängiger } \\
\text { Erwerbstätigkeit der Person }\end{array}$ & 35 & 25092 EUR & 8782 EUR \\
\hline Gesamtkosten & & & 103982 EUR \\
\hline \multicolumn{4}{|l|}{ Einnahmen. Einsparungen } \\
\hline Einsparungen Sozialleistungen & 100 & 97334 EUR & 97334 EUR \\
\hline Einkommensteuern - Unternehmergehalt & 49 & 31939 EUR & 15650 EUR \\
\hline Einkommensteuern - angestellte Personen & 4 & 75276 EUR & 3011 EUR \\
\hline Rückzahlung Sozialleistungen & & & 2266 EUR \\
\hline Rückzahlung Darlehen & 54 & 8574 EUR & 4630 EUR \\
\hline Leistungen gesamt & & & 122891 EUR \\
\hline Nettonutzen & & & 18909 EUR \\
\hline
\end{tabular}

Quelle: Ecorys, 2011. 


\section{Voraussetzungen für die Übertragung}

Um eine ähnliche Maßnahme in anderen Ländern einzuführen, müssen die nationalen Behörden sicherstellen, dass die folgenden Voraussetzungen erfüllt sind:

1. Sicherstellung eines soliden Rechtsrahmens. Für die wirtschaftlichen Aktivitäten, die die Klienten des Programms aufzunehmen beabsichtigen (d. h. selbstständige Erwerbstätigkeit, Kleinst- oder Kleinunternehmen), muss ein Rechtsrahmen gegeben sein, der verhindert, dass die Klienten sofort bestraft werden (durch den Verlust des Anspruchs auf Sozialleistungen), sobald sie sich für die Aufnahme einer selbstständigen Erwerbstätigkeit entscheiden.

2. Bereitstellung von ausreichend Ressourcen für eine umfassende Unterstützung. Die Maßnahme muss durch dieZuteilungvon ausreichend finanziellen Mitteln unterstützt werden.Es müssen beträchtliche Mittel für alle Bestandteile zugeteilt werden: Einkommensunterstützung, Wirtschaftsförderungsdienste, Kosten der Umsetzung der Geschäftspläne, Finanzierung der selbstständigen Erwerbstätigkeit.

3. Kapazitätsaufbau bei den Durchführungspartnern. Werden Stadtverwaltungen oder externe lokale Organisationen mit der Programmverwaltung betraut, ist darauf zu achten, dass diese entsprechend geschult sind und die nötigen Kapazitäten besitzen. Die Partner sollten eine klare Vorstellung von den Personen haben, die von den Dienstleistungen profitieren könnten, und praktische Kenntnisse über die Qualität möglicher (privatwirtschaftlicher und halbstaatlicher) Agenturen besitzen, die die Wirtschaftsförderungsdienste bereitstellen. Sie sollten außerdem in der Lage sein, Ausschreibungsverfahren zur Beauftragung lokaler Dienstleister (transparent) zu verwalten. Allerdings ist es wichtig, Höchstpreise für die Dienstleistungen festzulegen, die zentral bereitgestellt werden, um Ausschreibungsverfahren und Verhandlungsprozesse zu erleichtern. So könnten sich die lokalen Akteure auf die Qualität der zu erbringenden Dienstleistungen konzentrieren.

4. Entwicklung eines umfassenden Überwachungssystems. Da viele verschiedene Partner beteiligt sind, ist es wichtig, Buchhaltungs- und Verwaltungssysteme zu organisieren und einzurichten, um die Tätigkeiten $\mathrm{zu}$ überwachen und die Darlehensvergabe $\mathrm{zu}$ beaufsichtigen. Dieses Überwachungssystem sollte auch Klientenverfolgungssysteme beinhalten.

5. Zusammenarbeit mit dem privatwirtschaftlichen (und halbstaatlichen) Sektor. Lokale Wirtschaftsförderungsdienstleister sollten genutzt werden, um Unternehmerschulungen und andere Dienstleistungen für die Unternehmensgründung und -entwicklung anzubieten. Dabei ist die Entwicklung eines Screening-Mechanismus von Bedeutung, um zu prüfen, ob diese Dienstleister auch über die erforderlichen Kompetenzen verfügen.

\section{Literatur}

Bobbezoo, M. (2010), „De kosten van Bedrijfsadvisering in Nederland, Een verkennend onderzoek“.

Ecorys, (2011), Bbz 2004: uit het startblok - Eindrapport, in Auftrag gegeben durch das Ministerium für Soziales und Beschäftigung, Rotterdam, Niederlande.

Ministeries van Economische Zaken, Landbouw \& Innovatie en Sociale Zaken en Werkgelegenheid (2011), „Evaluatie Microkredietpilots Eindrapport Opdrachtgever“, Rotterdam.

Werkwijzer Levensuatbaarheidsonderzoek en begeleiding Bbz (2013), Stichting Divosa, verfügbar unter www.divosa.nl (auf Niederländisch). 


\section{Reifer Unternehmer, Polen}

Diese Fallstudie stellt ein lokales Projekt vor, das Menschen zwischen 50 und 64 Jahren mit Schulungen, Coaching und Mentoring sowie Beihilfen bei der Unternehmensgründung unterstützte. Die Ziele des Projekts sowie dessen Hintergrund werden beschrieben und seine Funktionsweise erläutert. Zudem werden Daten zu seinen Auswirkungen dargelegt. Außerdem werden in der Beschreibung die Herausforderungen, mit denen die Projektleiter konfrontiert waren, sowie die Voraussetzungen für die Übertragung dieser Art von Projekt auf einen anderen Kontext erörtert.

\section{Ziele}

Dieses Projekt wurde von 2009 bis 2010 in Gdansk als Pilotprojekt durchgeführt. Sein Ziel war es, arbeitslose oder nicht erwerbstätige Ältere (50- bis 64-Jährige) bei der Unternehmensgründung zu unterstützen. Zudem unterstützt wurden Ältere, die von einer abhängigen in eine selbstständige Erwerbstätigkeit wechseln wollten.

Ziel des Projekts war die Verringerung der Arbeitslosigkeit in Gdansk unter den Menschen im Alter zwischen 50 und 64 Jahren, die Erhöhung der Anzahl der Unternehmensgründungen in Gdansk und die Förderung der Entwicklung einer unternehmerischen Einstellung in der lokalen Gemeinschaft.

\section{Hintergrund}

Dieses Projekt bot eine Gelegenheit zur unternehmerischen Initiative für Menschen zwischen 50 und 64 Jahren, die immer noch gern einen Beitrag zur Gesellschaft und zum Arbeitsmarkt leisten wollten, jedoch entlassen wurden oder in den Ruhestand getreten sind. Eine Unternehmensgründung bietet diesen Menschen eine Möglichkeit, zur Gesellschaft beizutragen und aktiv in ihrer Gemeinschaft mitzuwirken. Außerdem können sie auf diese Weise etwas Geld verdienen, um ihre Altersvorsorge zu ergänzen.

Für dieses Projekt bestanden drei Hauptgründe. Erstens sind Ältere unter den Arbeitslosen in Polen in der Tendenz überrepräsentiert - Menschen über 50 Jahren machen etwa $20 \%$ der Arbeitslosen aus. Diese Überrepräsentation ist mehreren Faktoren geschuldet, unter anderem strukturellen Veränderungen, die dazu führen, dass erfahrene Arbeitskräfte aufgrund ihres höheren Gehalts entlassen werden. Zweitens sind die Ersparnisse für den Ruhestand bei vielen Älteren nicht ausreichend. Daher müssen diese wieder erwerbstätig werden, nachdem sie eine Zeit lang aus dem Erwerbsleben ausgeschieden sind. Dies kann für Ältere schwierig sein, da sie häufig auf dem Arbeitsmarkt diskriminiert und zugunsten von jüngeren Kandidaten bei der Stellensuche übersehen werden. Drittens zielte das Projekt auch darauf ab, gegen Stereotype gegenüber Älteren in der Gesellschaft anzugehen. Es stellte unter Beweis, dass sich Ältere zu helfen wissen und Geld verdienen können und sich nicht auf die staatliche Sozialhilfe stützen müssen. 


\section{Aktivitäten}

Das Pilotprojekt wurde vom kommunalen Arbeitsamt in Gdansk ins Leben gerufen. Es stellte eine Reihe integrierter Angebote bereit, unter anderem Schulungen, Coaching und Mentoring, eine Beihilfe, Unternehmensberatung, ein Überbrückungsgeld und Möglichkeiten zur Netzwerkarbeit. Die Koordination des Projekts übernahmen Beamte im lokalen Arbeitsamt, während die Schulungen, das Coaching und die Beratungsleistungen von Unternehmensberatern aus dem Privatsektor bereitgestellt wurden.

Das Aufnahmeverfahren für das Projekt bestand aus einem schriftlichen Bewerbungsformular, einem Gespräch und einer fünfminütigen mündlichen Präsentation vor einem Ausschuss, der aus Beamten des Arbeitsamtes Gdansk bestand. Die schriftliche Bewerbung und die Kurzpräsentation sollten die Geschäftsidee beschreiben und skizzieren, wie der Bewerber seine Idee in ein nachhaltiges Unternehmen umzusetzen beabsichtigte. Der Ausschuss bewertete jede Bewerbung und Präsentation unter den Aspekten Kreativität, Innovationsstärke und Potential zur Entwicklung in ein nachhaltiges Unternehmen. Außerdem wurde ein Gespräch durchgeführt, um die entsprechende Berufserfahrung sowie die Qualifikationen des Bewerbers zu prüfen und zu bewerten.

Während der Dauer des Projekts hielten 120 Bewerber eine Präsentation vor dem Ausschuss, und 60 wurden für die Teilnahme an dem Projekt ausgewählt. Diese 60 Teilnehmer hatten unterschiedliche Hintergründe und wurden in drei Gruppen aufgeteilt: Arbeitslose, Nichterwerbstätige und Beschäftigte. Jede Gruppe erhielt 150 Stunden Schulung bestehend aus acht Modulen. In diesen Modulen wurden die Themen Finanzierung, Marketing, Buchhaltung, rechtliche Fragen, Unternehmensplanung und rechtliche Voraussetzungen für eine Unternehmensgründung behandelt. Außerdem enthalten waren Computerschulungen und psychologische Workshops. Die Schulungen wurden von privatwirtschaftlichen Schulungsorganisationen durchgeführt.

Während ihrer Teilnahme an den Schulungen konnten sich die Teilnehmer an professionelle Unternehmensberater wenden, die ihnen bei der Entwicklung ihrer Geschäftspläne halfen. Konkret boten die Berater den Teilnehmern Unterstützung bei der Ausarbeitung einer ausführlichen Beschreibung ihres Produkts bzw. ihrer Dienstleistung, einer Einschätzung des Marktpotenzials und konjunktureller Einflüsse sowie eines Investitions- und Marketingplans. Die Teilnehmer konnten jeweils bis zu vier Stunden an individueller Beratung erhalten.

Nach Abschluss der Schulung konnten die Teilnehmer eine finanzielle Unterstützung beantragen, sofern sie an mindestens $80 \%$ der Schulungstermine teilgenommen hatten. Die finanzielle Unterstützung umfasste eine einmalige Beihilfe von bis zu 40000 PLN (etwa 9650 EUR) sowie ein Überbrückungsgeld in Form von sechs monatlichen Zahlungen über 1250 PLN (etwa 300 EUR) zur Deckung der Lebenshaltungskosten, während das Unternehmen erste Einnahmen abwarf. Das Überbrückungsgeld konnte um weitere sechs Monate verlängert werden. Diese finanzielle Unterstützung wurde von einem Komitee vergeben, das aus Vertretern von Arbeitgeberorganisationen bestand. Während des gesamten Projekts erhielten 26 Teilnehmer eine finanzielle Unterstützung. Die Empfänger dieser Unterstützung konnten außerdem während der sechs Monate, in denen sie das Überbrückungsgeld bezogen, weitere zwölf Stunden an individueller Unterstützung durch die Mentoren und Ausbilder erhalten.

Zudem entwickelte das Arbeitsamt eine Unterstützung in Form von Coaching und Mentoring für eine individuelle Betreuung der Teilnehmer. Die Betreuer und Mentoren wurden durch Arbeitgeberorganisationen ausgesucht und erhielten einen kleinen Lohn. Anfangs beabsichtigte man, für das Projekt Betreuer und Mentoren in etwa demselben Alter wie die Projektteilnehmer einzusetzen. Allerdings war es nicht möglich, aus dieser Altersgruppe einen ausreichenden Pool an Betreuern und Mentoren aufzubauen. Die Betreuer und Mentoren wurden auf der Grundlage ihres Fachwissens den Teilnehmern zugeordnet. In einigen Fällen hatten die Betreuer und Mentoren an den Schulungen mitgewirkt und bereits eine Beziehung zu den Teilnehmern aufgebaut.

Die Schulungen führten zudem zur Entwicklung von Unternehmernetzwerken für die Teilnehmer. Die Teilnehmer der einzelnen Schulungskohorten verbrachten viel Zeit miteinander, was dazu führte, dass sie untereinander Verbindungen aufbauten. Während der Schulungen und auch im Anschluss unterstützten sich die Teilnehmer als Gleichgestellte gegenseitig. Die Ausbilder und Betreuer förderten 
zudem die Entwicklung der Netzwerke durch Weitervermittlungen während der Schulungs- und Beratungssitzungen.

\section{Projektfinanzierung}

Die Kosten für das zwei Jahre laufende Projekt betrugen insgesamt 1,99 Millionen PLN (etwa 480500 EUR). Finanziert wurde es durch den Europäischen Sozialfonds (ESF) (85\%) und die nationale Regierung (15\%).

\section{Herausforderungen}

Dieses Projekt stand drei großen Herausforderungen gegenüber. Erstens gestaltete sich die Auswahl der Teilnehmer schwierig, da viele von ihnen Geschäftsideen vorgelegt hatten, die nicht realisierbar waren. Grund dafür waren zum Teil unrealistische Geschäftsideen, aber auch eine Diskrepanz zwischen den Erfahrungen und Qualifikationen der Personen auf der einen und der vorgeschlagenen Geschäftsidee auf der anderen Seite.

Ferner war ursprünglich beabsichtigt, aus jeder der drei anvisierten Klientengruppen dieselbe Anzahlvon Projektteilnehmern aufzunehmen, d. h. ein Drittel Arbeitslose, ein Drittel Nichterwerbstätige und ein Drittel Wechsler von einer abhängigen in eine selbstständige Erwerbstätigkeit. Jedoch war die große Mehrheit der anfänglichen Bewerber nicht erwerbstätig. Darum passten die Projektträger ihre Auswahlmethoden an, um die Arbeitslosen zu erreichen.

Zweitens machten Schulungen einen erheblichen Anteil am Unterstützungsangebot aus. Obwohl alle Teilnehmer das in den Schulungsmodulen erworbene Wissen zu schätzen wussten, waren viele erpicht, sofort ihr Unternehmen zu gründen, und fühlten sich durch die Länge der Schulungskomponente behindert, die durchlaufen werden musste, um eine finanzielle Unterstützung sowie Coaching und Mentoring in Anspruch zu nehmen. Dies stellte die Projektleiter vor eine Herausforderung, da sie die Teilnehmer überzeugen mussten, sich an das Programm zu halten.

Und schließlich stützte sich das Projekt auf Mittel der Europäischen Union und wurde eingestellt, sobald diese Mittel ausliefen. Die Projektmanager hätten es gern weitergeführt, konnten allerdings keine andere Finanzierung finden.

\section{Auswirkungen}

Von den 120 Bewerbern des Projekts wurden 60 für eine Teilnahme an der Schulungskomponente ausgewählt. Aus dieser Gruppe erhielten 26 Menschen eine Beihilfe und ein monatliches Überbrückungsgeld. Weitere sieben Teilnehmer erhielten eine kleine Beihilfe aus einem lokalen Beschäftigungsfonds. Zusammengenommen gründeten die Projektteilnehmer 33 Unternehmen. Sämtliche der neu gegründeten Unternehmen waren 2014 immer noch aktiv, und einige von ihnen hatten weitere Vollzeitstellen geschaffen.

Das Projekt wurde für seine Innovationsstärke mit mehreren Preisen ausgezeichnet. Im Jahr 2010 erhielt das Projekt den Europäischen Unternehmerpreis in der Kategorie „Beste Investition in Menschen“ und 2011 in der Kategorie „Förderung des Unternehmergeistes“.

Das Projekt diente zudem als Grundlage für eine zwölfteilige Fernsehsendung. Dazu wurde jede einzelne Projektphase gefilmt. Diese Fernsehsendung sollte ältere Unternehmer inspirieren. Außerdem wurde das Programm in einem 30-minütigen Film zusammengefasst.

\section{Voraussetzungen für die Übertragung}

Das Projekt „Reifer Unternehmer“ ist ein Beispiel für die Wirkung, die eine lokale Initiative auf den Unternehmergeist einer Gemeinschaft haben kann. Um diese Erfahrung auf einen anderen Kontext zu übertragen, sollten die folgenden zentralen Erfolgsfaktoren bei der Anpassung und Umsetzung berücksichtigt werden:

1. Ernennung eines starken Projektleiters. Der Projektleiter ist für den Erfolg des Projekts von zentraler Bedeutung, denn er ist verantwortlich für die Entwicklung der Unternehmerschulungen, den Aufbau eines Ausbilderpools, die Einbeziehung von Betreuern und Mentoren, die Werbung für das Angebot, 
um Teilnehmer anzuziehen, und die Koordination der Teilnehmer durch alle Unterstützungsphasen hindurch. Er ist außerdem für die Mittelbeschaffung zuständig, um sicherzustellen, dass ein gewisses Maß an finanzieller Unterstützung (d. h. Beihilfe und eine Zuwendung) bereitgestellt werden kann.

2. Entwicklung des Schulungsprojekts. Reifer Unternehmer umfasst 150 Stunden an Unternehmerschulungen für die Teilnehmer. Diese Schulungen müssen unbedingt an den lokalen Wirtschaftskontext und die Bedürfnisse der Teilnehmer angepasst werden. Würde dieses Projekt beispielsweise als "Junger Unternehmer" angeboten, müsste der Schulungsbestandteil an die Bedürfnisse und Herausforderungen junger Menschen angepasst werden.

3. Sicherung der finanziellen Unterstützung. Das, was dieses Projekt unter anderem attraktiv macht, ist das Angebot einer Beihilfe für einige der Kandidaten, um ihnen bei der Unternehmensgründung zu helfen, sowie einer Zuwendung, um sie während der ersten sechs Monate ihrer selbstständigen Erwerbstätigkeit bei der Deckung der Lebenshaltungskosten zu unterstützen. Um eine solche finanzielle Unterstützung anbieten zu können, müssen beträchtliche finanzielle Ressourcen vorhanden sein, vor allem bei einer großen Teilnehmeranzahl. Der Projektmanager muss Mittel von der nationalen Regierung beschaffen und auf EU-Ebene nach Möglichkeiten suchen, unter anderem beim ESF.

4. Aufbau eines Pools von Ausbildern, Beratern, Betreuern und Mentoren. Die Ausbilder, Betreuer und Mentoren sind die Fachleute, die die Unterstützung bereitstellen. Der Projektmanager muss ein Verfahren festlegen, mit dem privatwirtschaftliche (und halbstaatliche) Organisationen ermittelt werden, die die Schulungen durchführen können. Dieses Verfahren könnte in Form einer offenen Ausschreibung erfolgen. Darüber hinaus müssen die Berater, Betreuer und Mentoren nach der Unternehmensgründung maßgeschneiderte, individuelle Unterstützung leisten. Ausbilder könnten diese Rolle auch übernehmen, oft ist es jedoch wirksamer, hierzu Unternehmer zu engagieren. Zwar zahlte „Reifer Unternehmer“ seinen Beratern, Betreuern und Mentoren ein Gehalt, doch viele Projekte in der Europäischen Union sichern sich die Mitarbeit Freiwilliger, die ihrer Gemeinschaft „etwas zurückgeben“ möchten.

5. Nutzung einer kreativen Kontaktaufnahme. Die Kontaktaufnahme kann häufig für den Erfolg des Projekts entscheidend sein, denn die Öffentlichkeit muss unbedingt über das Angebot informiert werden, um die Zielklienten dafür zu gewinnen. „Reifer Unternehmer“ setzte sowohl die üblichen Werbemöglichkeiten (z. B. Websites) als auch innovativere Ansätze der Mediennutzung (z. B. Fernsehsendungen) ein. Es ist für Projekte wichtig, Arten der Kontaktaufnahme zu nutzen, die ihre Zielklienten erreichen. Häufig besteht eine wirksame Methode darin, gemeinschaftsbasierte Medien und Champions einzusetzen, die unter den Zielklienten angesehen sind.

\section{Literatur}

Arbeitsamt in Gdansk (2011), „Der Reife Unternehmer - Zusammenfassung des Projekts zur Förderung des Unternehmertums unter Menschen über 50 Jahre“, verfügbar unter: www.pup.gda.pl. 


\title{
Unternehmerisch in die Geschäftswelt, Slowenien
}

\author{
Diese Fallstudie stellt ein Projekt vor, das junge Menschen bei der Unternehmensgründung \\ unterstützt. Die Ziele des Projekts sowie dessen Hintergrund werden vorgestellt und \\ seine Aktivitäten beschrieben, darunter Unternehmerschulungen, Coaching und \\ Mentoring, Unternehmensberatung und eine Zuwendung. Zudem werden Daten zu \\ seinen Auswirkungen vorgestellt. Ferner erörtert die Fallstudie die Herausforderungen \\ bei der Gestaltung und Durchführung des Programms und die Voraussetzungen für \\ seine Übertragung auf einen anderen Kontext.
}

\section{Ziele}

„Unternehmerisch in die Geschäftswelt“ (Podjetno v suet podjetnistva) wurde ins Leben gerufen, um der hohen Arbeitslosenquote unter den Menschen unter 35 Jahren mit einem abgeschlossenen grundständigen oder postgradualen Studium (unabhängig von der Hochschule, dem Studiengang oder der Art des Studiums) entgegenzuwirken. Es sollte ausgewählten Teilnehmern ermöglichen, eine Geschäftsmöglichkeit zu erkennen und zu nutzen und eine selbstständige Erwerbstätigkeit aufzunehmen, ein eigenes Unternehmen zu gründen oder eine Stelle bei einem sonstigen Arbeitgeber zu finden. Das Projekt wurde mit den folgenden wichtigen Zielen im Blick gestartet:

- Verringerung der Jugendarbeitslosigkeit;

- Erweiterung der unternehmerischen Fähigkeiten; und

- Schaffung hochwertiger Arbeitsplätze.

Das Projekt zahlte über die regionalen Entwicklungsagenturen sechs Monate lang ein Gehalt aus, während die Teilnehmer ein intensives Schulungsprogramm zu den Themen Unternehmertum, Marketing, Vertrieb, Buchhaltung und Finanzverwaltung durchliefen oder Coaching und Mentoring in Anspruch nahmen, um eine bestimmte Geschäftsidee weiterzuentwickeln. Am Ende des Programms wurden den Teilnehmern noch ein weiteres Jahr lang Beratungsdienste zur Verfügung gestellt. Das Projekt wurde zwischen 2009 und 2012 in der Region Zasavje als Pilotprojekt getestet und in den Jahren 2013 und 2014 national umgesetzt.

\section{Hintergrund}

Das Projekt wurde anfangs auf regionaler Ebene in Zasavje, der kleinsten Region Sloweniens, umgesetzt. Das ehemals hoch entwickelte Bergbau- und Industriegebiet sah sich aufgrund nachteiliger Wettbewerbs- und Marktentwicklungen mit einem rapiden Verlust von Arbeitsplätzen konfrontiert. Angesichts eingeschränkter Investitionen in neue Unternehmen und eines begrenzten Wachstums 
der aktiven Unternehmen bot das Unternehmertum junger Menschen eine potenzielle Lösung, um die Entwicklung der Region wieder neu aufleben zu lassen.

Junge Menschen in Slowenien stehen nach wie vor beim Einstieg in den Arbeitsmarkt vor Herausforderungen, die sie daran hindern, eine angemessene Beschäftigung zu finden. Es werden zwar Ressourcen und Energie in ihre Bildung investiert, doch eine anhaltende Arbeitslosigkeit schadet langfristig ihren sozialen und wirtschaftlichen Aussichten und drängt sie in verschiedene Formen prekärer Arbeit. Zu den Hauptgründen für ihre Schwierigkeiten beim Einstieg in den Kernarbeitsmarkt zählen:

- fehlende Arbeitserfahrung;

- eine Unausgewogenheit zwischen dem Angebot an gebildeten Arbeitskräften und der Nachfrage nach gebildeten Arbeitskräften auf dem Stellenmarkt;

- die Vorherrschaft kurzfristiger oder sporadischer lokaler Beschäftigungsangebote; und

- ein Mangel an neuen Arbeitsplätzen.

Entsprechend wollte man mit der Initiative die unzureichende Übereinstimmung zwischen den im Hochschulsystem generierten und den seitens der Arbeitgeber benötigten Fähigkeiten angehen und eine politische Lücke (d. h. die geringe Anzahl von Arbeitsmarktmaßnahmen für junge Absolventen) schließen.

\section{Aktivitäten}

Das Projekt stellte Schulungen, Mentoring und individuelle Unternehmensberatung bereit, um den Teilnehmern beim Erwerb der Kernkompetenzen zu helfen, die sie für die Entwicklung und Umsetzung einer Geschäftsidee benötigten. Ziel war es, dass die Teilnehmer innerhalb eines Jahres nach Abschluss der Schulungen in der Lage sein sollten, ein Unternehmen zu gründen, eine selbstständige Erwerbstätigkeit aufzunehmen oder eine abhängige Erwerbstätigkeit zu finden. Um infrage zu kommen, mussten potenzielle Kandidaten die folgenden Kriterien erfüllen:

- Sie waren als arbeitslose Arbeitsuchende gemeldet;

- sie waren jünger als 35 Jahre;

- sie hatten einen Hochschul- oder Masterabschluss oder einen Doktorgrad;

- sie hatten eine Geschäftsidee; und

- sie hatten einen dauerhaften Wohnsitz in der Region.

Das Projekt wurde in drei Phasen umgesetzt: i) als Pilotprojekt in der Region Zasavje von 2009 bis 2012, ii) als nationales Projekt, das 2013 von jeder der zwölf regionalen Entwicklungsagenturen umgesetzt wurde, und iii) als größeres nationales Projekt, das 2014 von jeder der zwölf regionalen Entwicklungsagenturen mit einer größeren Teilnehmerzahl umgesetzt wurde. Die ursprüngliche Zielgruppe für das Pilotprojekt in der Region Zasavje umfasste 40 Menschen. Die Zielgruppe des nationalen Projekts umfasste 240 Menschen im Jahr 2013 und 360 Menschen im Jahr 2014.

Die erste Phase wurde ausschließlich von der regionalen Entwicklungsagentur Zagorje in der Region Zasavje umgesetzt, die durch die Stadtverwaltung Zagorje ob Savi unterstützt wurde. Das nationale Projekt wurde mit Unterstützung des slowenischen Ministeriums für Arbeit, Familie, Soziales und Gleichstellung von allen zwölf regionalen Entwicklungsagenturen in Slowenien umgesetzt. Obwohl jede regionale Entwicklungsagentur bei der Umsetzung des Projekts in ihrer jeweiligen Region ein gewisses Maß an Flexibilität hatte, übernahm die regionale Entwicklungsagentur in Zagorje ob Savi als leitender Umsetzungspartner eine übergeordnete Überwachungsfunktion. Sie war auch für die Berichterstattung und die finanzielle Rechenschaftspflicht verantwortlich.

In allen Projektphasen wurde derselbe Ansatz verfolgt, der in der anfänglichen Umsetzungsphase gestaltet wurde und folgende Hauptphasen umfasste:

1. Aufforderung zur Bewerbung und Auswahlverfahren;

2. Beschäftigung in den regionalen Entwicklungsagenturen und umfassende Schulungen während eines bestimmten Zeitraums; und

3. unterstützende Beratung während eines Jahres nach Programmende. 
Die Aufforderungen zur Bewerbung wurden zeitgleich von allen regionalen Entwicklungsagenturen im slowenischen Amtsblatt und online veröffentlicht, um ausreichend Interesse zu wecken, damit für jede Umsetzungsrunde zehn Teilnehmer ausgewählt werden konnten. In der Anfangsphase ergingen vier Aufforderungen zur Bewerbung und für jedes sechsmonatige Beschäftigungs- und Schulungsprogramm wurden zehn Teilnehmer ausgewählt. Das Beschäftigungs- und Schulungsprogramm wurde im Rahmen der nationalen Projekte in den Jahren 2013 bzw. 2014 auf fünf Monate (zwei Aufforderungen pro Jahr) bzw. vier Monate (drei Aufforderungen pro Jahr) verkürzt. Diese Verkürzung des Beschäftigungs- und Schulungsprogramms war das Ergebnis der Entscheidung, mit den denselben finanziellen Ressourcen weitere Teilnehmergruppen aufzunehmen.

Um sich für das Projekt zu bewerben, mussten die Bewerber ein Bewerbungsformular einreichen, einen Fragebogen ausfüllen und ihren Lebenslauf, ihre Abschlusszeugnisse und eine Bestätigung ihrer Arbeitslosigkeit von der Arbeitsverwaltung vorlegen. Zusätzlich zu den grundlegenden persönlichen Informationen mussten die Bewerber in dem Fragebogen Angaben zu ihren Fähigkeiten und Interessen, beruflichen Zielen, ihrer unternehmerischen Motivation und ihren Geschäftsideen sowie zu Möglichkeiten für deren Entwicklung machen. Die Anzahl der Bewerber war von Region zu Region unterschiedlich, wobei in kleineren Gemeinschaften das Interesse am geringsten war und größere Städte wie Ljubljana und Maribor am meisten Interesse zeigten. Die Evaluierungsgremien der regionalen Entwicklungsagenturen bestanden jeweils aus zwei Mitarbeitern, einem externen Mentor und einem Mitarbeiter der Arbeitsverwaltung, der zwischen 20 und 50 Kandidaten (pro Region) zu einem Gespräch einlud. Die Hauptkriterien für die Auswahl der Projektteilnehmer waren das unternehmerische Potenzial, das Wissen, die Motivation und die Interessen der Kandidaten und vor allem ihre Geschäftsidee und der Handlungsspielraum für ihre Verwirklichung.

Die zehn in jeder Region ausgewählten Teilnehmer wurden von den regionalen Entwicklungsagenturen als öffentliche Bedienstete eingestellt und erhielten für die Dauer des umfassenden Schulungsprogramms, an dem sie teilnahmen und das von Mitarbeitern der Agenturen und externen Mentoren geleitet wurde, einen VollzeitvertragmitMindestlohn(789EUR monatlich imJahr 2014).Jede der regionalen Entwicklungsagenturen setzte Ausbilder, in der Regel erfahrene Agenturmitarbeiter, ein, um die Schulungsmodule zu koordinieren und durchzuführen, die mit ihrem Fachbereich zusammenhingen. Außerdem engagierte jede Agentur einen externen Mentor für jede Teilnehmergruppe, der bis zu 28 Stunden pro Monat während des gesamten Schulungsprogramms jeder Gruppe spezielle Schulungen durchführte. Üblicherweise handelte es sich dabei um einen erfolgreichen lokalen Unternehmer oder einen Rechtsanwalt mit Erfahrungen im Handelsrecht. Externe Mentoren wurden für ihre Dienste mit 900 EUR pro Monat vergütet.

Das Schulungsprogramm war so angelegt, dass es den Teilnehmern täglich Wissen in allen mit dem Unternehmertum zusammenhängenden Themen vermittelte, darunter Einführung in das Unternehmertum, Erstellung eines Geschäftsmodells, Geschäftspläne, Finanzierung, Handelsrecht, Beschäftigung, Buchhaltung, Marketing, Vertrieb, IKT, Netzwerkarbeit und soziales Unternehmertum. Schwerpunkte des Schulungsprogramms waren zu Anfang allgemeine Themen und Gruppenarbeit. Mit der Zeit wurden verstärkt individuelle Schulungen und Coaching für jeden Teilnehmer und seine Geschäftsidee durchgeführt. Zusätzlich luden die regionalen Entwicklungsagenturen abhängig von den Bedürfnissen der jeweiligen Teilnehmergruppe andere lokale Unternehmer und Fachleute ein, die Schulungsmodule durchführten und darin ihre Erfahrungen und Kenntnisse weitergaben.

Sowohl die internen Mitarbeiter der regionalen Entwicklungsagenturen als auch die externen Mentoren stehen den Teilnehmern noch ein Jahr nach dem erfolgreichen Abschluss des Schulungsprogramms mit Beratungsdiensten zur Entwicklung ihrer Geschäftsidee zur Verfügung. Allerdings ist für diese Unterstützung keine Struktur vorgegeben - sie hängt von den besonderen Bedürfnissen des Teilnehmers und der Verfügbarkeit der Mentoren ab.

\section{Projektentwicklung}

Die Projektkonzeption hat sich seit dem anfänglichen Pilotprojekt in Zasavje geringfügig verändert:

- Die Dauer der Beschäftigung und der Schulungen hat sich von ursprünglich sechs Monaten je Gruppe während der ersten landesweiten Phase auf fünf Monate und schließlich bei der Durchführung im Jahr 2014 auf vier Monate je Gruppe verkürzt, um in jeder Durchführungsphase mehr Teilnehmer 
aufnehmen zu können und die Kosten überschaubar zu halten. Der Umfang der Schulungen und der Unterstützung ist gleich geblieben.

- In den anschließenden Projektphasen wurde das Schulungsprogramm in der Art umgestaltet, dass es mehr spezifische und individuelle Schulungen enthielt, die stärker auf die Bedürfnisse der Teilnehmer zugeschnitten waren.

- Die Schulungsprogramme wurden von den jeweiligen regionalen Entwicklungsagenturen abhängig von ihren jeweiligen Teilnehmergruppen verändert und so gestaltet, dass sie Veränderungen in der wirtschaftlichen Entwicklung, den IKT und der Entwicklung von Crowdfunding usw. berücksichtigten.

- Die regionalen Entwicklungsagenturen bemühen sich um die Einbindung von mehr externen Experten (Unternehmern und verschiedenen Fachleuten) in das Schulungsprogramm, die ehrenamtlich tätig sind.

- Nach Abschluss des Schulungsprogramms können sich die Teilnehmer bei bestehenden Unterstützungsprogrammen, die die slowenische Regierung eingerichtet hat, um eine finanzielle Unterstützung für ihr Start-up bewerben, doch das Projekt selbst stellt Teilnehmern nach Abschluss des Schulungsprogramms keine solche Unterstützung bereit.

Seit das Projekt 2013 auf alle regionalen Entwicklungsagenturen ausgeweitet wurde, hat das Umsetzungsteam außerdem eine nationale Projektkonferenz für alles Teilnehmer ausgerichtet, auf der sie Erfahrungen austauschen, Netzwerkarbeit betreiben und potenziell neue Kooperationen in die Wege leiten können.

\section{Projektfinanzierung}

Das anfängliche Pilotprojekt wurde gemeinsam von der regionalen Entwicklungsagentur Zagorje (57\%), dem Europäischen Fonds für regionale Entwicklung (28\%) und der Stadtverwaltung von Zagorje ob Savi (15\%) mit einem Haushalt von 604900 EUR finanziert.

Das erste nationale Projekt wurde unter dem Titel „Unternehmerisch in die Geschäftswelt 2013“ in der Zeit vom 1. November 2012 bis zum 30. April 2014 mit einem Gesamthaushalt von 3816534 EUR durchgeführt. Es wurde über den Europäischen Sozialfonds (ESF) (85\%) von der Europäischen Union sowie vom Ministerium für Arbeit, Familie, Soziales und Gleichstellung (15\%) finanziert. Das Projekt wurde im Rahmen des operationellen Programms zur Förderung der Humanressourcen für den Zeitraum 2007-2013, Prioritätsachse 1: „Förderung von Unternehmertum und Anpassungsfähigkeit“, 1.2 politische Priorität „Schulungen und Bildung für Wettbewerbsfähigkeit und Beschäftigungsfähigkeit" durchgeführt.

Die zweite Phase des nationalen Projekts wurde in der Zeit vom 15. November 2013 bis zum 30. August 2015 umgesetzt. Der Projekthaushalt wird auf 4702038 EUR geschätzt und über den ESF (85\%) von der Europäischen Union sowie vom slowenischen Ministerium für Arbeit, Familie, Soziales und Gleichstellung (15\%) kofinanziert.

\section{Herausforderungen}

Im Laufe seiner Entwicklung war das Projekt mit einigen Herausforderungen konfrontiert. Erstens ist es zuweilen schwierig, potenzielle Kandidaten für das Projekt zu gewinnen. Um eine ausreichende Anzahl von Kandidaten mit hochwertigen Geschäftsplänen zu erreichen, kann das Evaluierungsgremium entschließen, während der Evaluierung von Bewerbungen einige der Zugangsvoraussetzungen zu lockern, wenn eine außergewöhnliche Geschäftsidee vorgelegt wird. Außerdem werden, wenn jede Gruppe weniger als zehn erfolgreiche Bewerber fasst, Bewerber in die aktuelle Teilnehmergruppe eingeladen, die bei einer früheren Aufforderung keinen Erfolg hatten, um die Gruppe zu vergrößern.

Zudem stellt es eine Herausforderung dar, in den Schulungen eine angemessene Themenauswahl für die jeweiligen Unternehmergruppen anzubieten. Jede regionale Entwicklungsagentur kann das Schulungsprogramm flexibel an ihre jeweilige Teilnehmergruppe anpassen. Dies sorgt für Flexibilität und ermöglicht die Anpassung an die Anforderungen des regionalen Arbeitsmarktes. 


\section{Auswirkungen}

Die Erfolgsquote wird über ein Datenerhebungsformular der Arbeitsverwaltung und eine Anmeldebestätigung von der Agentur für öffentliche Register und Dienstleistungen überwacht. Angesichts der relativ kleinen Teilnehmergruppen pro Aufforderung zur Bewerbung entwickelte das Projekt außerdem Online-Kommunikationstools für die Teilnehmer und die Umsetzungspartner, und das Projektteam steht interessierten Projektteilnehmern mit Unterstützung und Fortschrittsverfolgung zur Verfügung, nachdem diese offiziell aus dem Projekt ausgestiegen sind.

Während der ersten Phase von 2009 bis 2012 haben sich 97 arbeitslose junge Menschen aus der Region Zasavje für eine Teilnahme an dem Projekt beworben. Das Projekt wählte 40 Teilnehmer aus, und man rechnete damit, dass es zur Gründung von zwei neuen Unternehmen führen und zehn weiteren Menschen bei der Sicherung einer abhängigen Beschäftigung unterstützen würde. Leider bestand in diesem Zeitraum nicht die Verpflichtung, sich mit einem Beschäftigungsnachweis bei der regionalen Entwicklungsagentur zurückzumelden. Auf der Grundlage von Befragungen schätzte das Projektumsetzungsteam, dass die erste Phase zur Gründung von zehn neuen Unternehmen in der Region Zasavje führte und zehn Teilnehmer (von 40) einen Arbeitsplatz als Angestellte finden konnten.

Im Rahmen der ersten Aufforderung zur Bewerbung an dem nationalen Projekt im Januar 2013 bewarben sich 754 Arbeitslose um eine Teilnahme. Im Anschluss an die zweite Aufforderung zur Bewerbung gingen bis Ende 2013 insgesamt 1246 Bewerbungen ein. Nach der ersten Aufforderung wurden 130 und nach der zweiten 120 Teilnehmer ausgewählt (d. h. insgesamt 250). Laut den verfügbaren Daten (Stand 31. Dezember 2014) haben 134 (62,8\%) der Teilnehmer an der ersten landesweiten Gruppe eine Beschäftigung gefunden oder ein Unternehmen gegründet.

Im Jahr 2014 konnte das Projekt drei Aufforderungen zur Bewerbung veröffentlichen, da die Schulungsdauer auf vier Monate reduziert worden war. Es nahmen 360 Menschen teil.

Die entsprechenden Ergebnisse sind in Tabelle 18.1 zusammengefasst.

\section{Tabelle 18.1. Schlüsselkennzahlen für die drei Projektphasen von „Unternehmerisch in die Geschäftswelt“}

\begin{tabular}{lcccc}
\hline \multicolumn{1}{c}{ Umsetzungsphase } & Haushalt in Euro & Teilnehmer & Kosten pro Teilnehmer & $\begin{array}{c}\text { Erfolgsquote } \\
\text { (d. h. selbstständige oder } \\
\text { abhängige Erwerbstätigkeit) }\end{array}$ \\
\hline $\begin{array}{l}\text { Lokales Projekt } \\
\begin{array}{l}2009-2012 \\
\text { Nationales Projekt }\end{array}\end{array}$ & 604900 & 40 & 15122 EUR & $47 \%$ \\
$\begin{array}{l}2013 \\
\text { Nationales Projekt }\end{array}$ & 3816534 & 250 & 15266 EUR & $63 \%$ \\
2014 & 4702038 & 360 & 13061 EUR & $60 \%$ \\
\hline
\end{tabular}

\section{Voraussetzungen für die Übertragung}

Dieses Projekt begann als lokales Pilotprojekt, das erfolgreich in ein nationales Projekt ausgeweitet wurde, nachdem es sich vielversprechend entwickelte. Die Erfahrung bringt viele Erkenntnisse und deutet darauf hin, dass beim Versuch, einen ähnlichen Ansatz in anderen Kontexten einzuführen, die folgenden kritischen Aspekte berücksichtigt werden sollten:

1. Lokale Entwicklung von Unterstützung, um die Klientenbedürfnisse zu erfüllen. Dieses Projekt begann als Pilotprojekt in einer Region Sloweniens. Es konnte den nationalen Einrichtungen zeigen, was erreicht und wie es erreicht werden konnte. Diese lokale Herangehensweise ermöglichte nicht nur, dass eine Unterstützung entwickelt werden konnte, die die Klientenbedürfnisse erfüllte, sondern reduzierte auch das Risiko, beträchtliche öffentliche Mittel für erste Versuche mit einem nationalen Ansatz auszugeben. Damit dieser Ansatz erfolgreich übertragen werden kann, müssen die nationalen Behörden diesem „Bottom-up“-Ansatz offen gegenüberstehen und über Systeme 
verfügen, um Initiativen auszuwerten und sie nach der Möglichkeit einer Ausweitung auf nationale Ebene zu filtern.

2. Sicherstellen, dass die Ziele angemessen sind. Da das Programm gleichmäßig auf die regionalen Entwicklungsagenturen aufgeteilt war, war es für einige Regionen schwieriger als für andere, die zugewiesenen Plätze zu besetzen. Das führte dazu, dass in Regionen mit weniger Bewerbern erfolglose Bewerber aus vorherigen Runden aufgenommen und die Altersgruppen nach unten und nach oben ausgeweitet werden mussten. Dies hat wahrscheinlich Einfluss auf die letztendlichen Auswirkungen, könnte aber ausgeglichen werden, indem die Zuteilung pro Region auf Basis der Einwohnerzahl erfolgt.

3. Sicherung finanzieller Ressourcen, um Gehälter/Zuwendungen zu zahlen. Ein wichtiges und neuartiges Merkmal dieses Projekt bestand darin, dass die regionalen Entwicklungsagenturen dieTeilnehmer vier bis sechs Monatelangzum Mindestlohn einstellen konnten. Die Notwendigkeit dieses Merkmals hängt von den Eigenschaften der Teilnehmer und der Verfügbarkeit anderer Unterstützungsleistungen im Sozialhilfesystem ab. Dieses Merkmal sorgte einerseits für eine gewisse Einkommensunterstützung und unterstützte darüber hinaus die Entwicklung starker Arbeitsgewohnheiten und erleichterte den Kontakt mit einem breiten Spektrum an Geschäftsexperten und Unternehmern. Dies steigert die Motivation und stärkt Unternehmernetzwerke.

4. Einräumung von Flexibilität bei der Umsetzung. Die nationalen Einrichtungen müssen der Möglichkeit von Abweichungen offen gegenüberstehen, die einen Mehrwert für die Teilnehmer generieren, statt auf einem Universalkonzept zu bestehen, das häufig von Finanzierungsmechanismen gefordert wird. Allerdings hat der Grad an Flexibilität Folgen für das Überwachungs- und Evaluierungssystem und muss bei der Gestaltung dieser Systeme berücksichtigt werden.

5. Bereitstellung einer gewissen finanziellen Unterstützung. In den ersten zwei Runden des Projekts war keinerlei finanzielle Unterstützung der potenziellen Unternehmer vorgesehen. Allerdings wäre die Erfolgsquote der ersten zwei Runden vermutlich etwas höher ausgefallen und überhaupt die Qualität der Bewerber besser gewesen, hätte es bereits die Beihilfe in Höhe von 3500 EUR gegeben, die für die dritte Runde eingeplant wurde.

\section{Literatur}

GEM (2013), „Spregledan podjetniški potencial mladih“ (slowenischer Nationalbericht), verfügbar unter: www.gemconsortium.org.

Jauni poziv za uključitev v operacijo Podjetno v suet podjetništva (2014), verfügbar unter: www.rcr-zasavje.si/ uploads/razpisi\%202014/jauni\%20poziv\%20poprauki/drugi\%20jauni\%20poziv\%20PVSP\%202014.doc.

Projekti: Podjetno v suet podjetništua 2014 (2014), verfügbar unter: www.rcr-zasavje.si/si/projekti/17-podjetno-vsuet-podjetnistua-2013.html.

Projekti: Podjetno v suet podjetništva (2014), verfügbar unter: www.rcr-zasavje.si/si/projekti/13-podjetno-vsuet-podjetnistva.html. 


\title{
Startrampen für Beschäftigung und Unternehmertum, Spanien
}

\begin{abstract}
DieseFallstudiestelltein Beispielfür eineUnterstützungfür dieUnternehmensentwicklung für Arbeitslose vor, die durch eine Unternehmensgründung für sich selbst einen Arbeitsplatz schaffen möchten. Es werden die Ziele des Projekts und dessen Hintergrund sowie seine Funktionsweise beschrieben und die Auswirkungen des Projekts anhand von Daten zu ersten Ergebnissen illustriert. Außerdem werden die bei der Bereitstellung dieser Unterstützung sichtbar werdenden Herausforderungen und die Voraussetzungen erörtert, die für die Übertragung dieses Ansatzes auf einen anderen Kontext gegeben sein müssen.
\end{abstract}

Ziele

Das Programm „Startrampen für Beschäftigung und Unternehmertum“ (Las Lanzaderas de Empleo y emprendimiento solidario) zielt darauf ab, Langzeitarbeitslosen (d. h. Menschen, die seit mindestens zwölf Monaten arbeitslos sind) durch Unternehmer-„Startrampen“ („lanzaderas“) beim Einstieg in eine selbstständige Erwerbstätigkeit oder bei der Rückkehr in die Beschäftigung zu helfen. Es handelt sich um ein integriertes Programm, das Orientierungshilfen gibt, Teilnehmer an Geschäftsexperten weitervermittelt und Schulungen, Coaching und Mentoring sowie psychologische Unterstützung anbietet. Seine Ziele sind folgende:

1. Die Verbesserung und Stärkung der Beschäftigungsfähigkeit der Teilnehmer durch eine Diagnose und Analyse ihrer Fähigkeiten und die Erstellung eines Entwicklungsplans, der auf die Bedürfnisse des jeweiligen Teilnehmers zugeschnitten ist.

2. Die Wiederherstellung oder Stärkung des Selbstwertgefühls durch gegenseitige Unterstützung, die die Entwicklung von emotionaler Intelligenz ermöglicht. Dazu wird ein Schwerpunkt auf Teamarbeit gelegt, eine der gefragtesten Meta-Kompetenzen auf dem Arbeitsmarkt und eine wichtige Grundlage für die persönliche Entwicklung.

3. Die Entwicklung eines Beziehungsnetzwerks, um die Unternehmensgründung zu unterstützen und nach Möglichkeiten Ausschau zu halten.

4. Die Weiterentwicklung der unternehmerischen Fähigkeiten, damit die Teilnehmer ihre eigenen Projekte erarbeiten können. Das Ziel besteht darin, sie dazu zu ermutigen, die Möglichkeit einer Tätigkeit als Unternehmer in Erwägung zu ziehen, aber auch als Angestellte ein kreatives und unternehmerisches Denken einzusetzen.

5. DieIntensivierungderVerbindungenzwischendenWirtschaftsförderungsdienstleistern.Die,Startrampen“ bilden ein offenes System, das Organisationen verbindet. Geschäftsexperten und Unternehmer können einen Beitrag leisten, indem sie ihr Wissen teilen und an den Programmveranstaltungen mitwirken. 


\section{Hintergrund}

In vielen südlichen Ländern der Europäischen Union, insbesondere in Spanien, ist die Arbeitsmarktlage in jüngster Zeit durch eine allgegenwärtige Arbeitslosigkeit gekennzeichnet. Im Jahr 2014 erreichte die Arbeitslosenquote in Spanien 24,5\% (Eurostat, 2015).

Dieses laufende Projekt wurde 2013 von dem sozialen Unternehmer José María Pérez gestartet, der der Ansicht war, dass die aktive Arbeitsmarktpolitik nicht ausreiche, um mit der Größenordnung des Problems umzugehen, und dass Arbeitslose stärker an der Gesellschaft teilhaben müssten, um wieder in den Arbeitsmarkt einzusteigen. Die „Startrampen“ sind den Berufsschulworkshops nachempfunden, die Pérez 1985 gründete, um Handwerksmeistern dabei zu helfen, arbeitslosen jungen Menschen handwerkliche Fähigkeiten zu vermitteln und ihnen zu ermöglichen, Arbeit bei der Restaurierung historischer Stätten zu finden. Dieses Projekt ist gewachsen und wird aktuell über den Europäischen Sozialfonds (ESF) finanziert. Es breitete sich auch nach Lateinamerika aus, wo die Berufsschulworkshops nun in mehr als 17 Ländern durchgeführt werden. Heute haben fast 500000 Schüler in Spanien und Lateinamerika dieses Programm durchlaufen, und $80 \%$ von ihnen konnten mit Erfolg einen Arbeitsplatz für sich schaffen oder eine Beschäftigung finden.

Dieses Programm möchte dem Stigma der Arbeitslosigkeit entgegenwirken und sie als Möglichkeit gestalten, die eigenen Fähigkeiten zu stärken und eine unternehmerische Denkweise zu entwickeln. Es verfährt nach einem kooperativen, unterstützenden Modell, bei dem die persönliche Entwicklung und Teamarbeit im Mittelpunkt stehen. Getreu der Auffassung, dass Isolation eine schädliche Wirkung auf den Menschen hat und seinen Antrieb und seine Kreativität untergräbt, zielt das Programm auch darauf ab, Arbeitslose von ihrer Isolation, Einsamkeit, Entmutigung und Unsichtbarkeit zu befreien und sie in die Lage zu versetzen, sichtbar und proaktiv zu sein. Es kombiniert Aktivitäten rund um die Stellensuche mit Unternehmerworkshops und stützt sich auf die kollektive Befähigung der Teilnehmer und eine gemeinsame soziale Vision. Seine Philosophie lautet: „Jede Bürde wird leichter, wenn sie von vielen getragen wird, und gute Ideen ziehen gute Gesellschaft an."

\section{Aktivitäten}

Das Programm basiert auf einer partnerschaftlichen Einstellung und wird in Anlehnung an ein kooperatives Gesellschaftsmodell durchgeführt. Es wird von einem Team aus 20 Freiwilligen verwaltet, die anhand von zwei Hauptkriterien ausgewählt werden: i) Sie sind seit mindestens einem Jahr arbeitslos, und ii) sie beweisen einen starken persönlichen Antrieb und Interesse, sich neuen Herausforderungen zu stellen.

Um sich bei dem Programm anzumelden, müssen die Teilnehmer zustimmen, in Gruppen zusammenzuarbeiten, sich selbst zu organisieren und als Hauptakteure hinter den verschiedenen Tätigkeiten zu stehen, die im Rahmen der „Startrampe“ organisiert werden. Es sind zwar keine besonderen Berufserfahrungen oder ein bestimmter Bildungshintergrund notwendig, um bei dem Programm mitzuwirken, doch die Teilnehmer sagen zu, sich gegenseitig zu schulen und ihre beruflichen Qualifikationen und unternehmerischen Fähigkeiten gemeinsam zu verbessern.

Die „Startrampen“ laufen zwischen fünf und neun Monaten (abhängig von der Teamgröße sowie von den Auflagen der Finanzierungseinrichtungen) und sind in vier Phasen aufgeteilt.

1. Die erste Phase besteht aus der „Diagnose“. Dabei ermitteln die Teilnehmer die Gefühle, die sie mit der Arbeitslosigkeit verbinden, und drücken diese aus, sie verstehen Veränderungen auf dem Arbeitsmarkt und lernen sich gegenseitig kennen, um eine Grundlage für die Teamarbeit zu schaffen.

2. In der zweiten Phase werden auf persönlicher und auf Teamebene spezifische Aktionspläne entworfen. Die Teilnehmer beginnen, an Schlüsselkompetenzen zu arbeiten, die sie für die Unternehmensgründung oder die Stellensuche benötigen, und fangen an, die Umgebung zu analysieren und Geschäftsideen hervorzubringen, während sie an Schulungssitzungen teilnehmen.

3. In der dritten Phase soll die Interaktion mit der äußeren Umgebung gefördert werden. Die Teilnehmer nehmen an Netzwerkveranstaltungen teil und besuchen Firmen und Unternehmer, um die in der zweiten Phase entwickelten Ideen zu testen und weiterzuentwickeln. In dieser Phase arbeiten die Teilnehmer außerdem an ihren Geschäftsplänen. 
4. In der vierten Phase liegt der Schwerpunkt auf der Umsetzung der Einzel- und Gruppenpläne. Die einzelnen Teilnehmer konzentrieren sich auf ihre Geschäftstätigkeiten, und die Gruppen unterstützen sich gegenseitig und machen Pläne für die Fortführung des Netzwerks nach dem Ende der „Startrampe“. Außerdem erstelltjede Gruppe ein Handbuch bewährterVerfahren, das einer nachfolgenden „Startrampe“ zur Verfügung gestellt wird. Für alle „Startrampen“ wird eine Abschlussveranstaltung ausgerichtet.

Die Aktivitäten der „Startrampen“ finden fünfmal die Woche am Vormittag statt. Die ersten drei Wochentage sind Gruppensitzungen gewidmet, die von dem Betreuer geleitet werden. Diese Sitzungen konzentrieren sich auf die Entwicklung unternehmerischer Fähigkeiten, um den Teilnehmern bei der Vorbereitung auf die Unternehmensgründung zu helfen und ihre Beschäftigungsfähigkeit zu verbessern. $\mathrm{Zu}$ den Aktivitäten zählen Grundsatzreden, Firmenbesuche, Fallstudien, Evaluierungen geschäftlicher Möglichkeiten und Kreativitätsworkshops, bei denen Brainstorming, die Anpassung von Ideen und kreatives Denken im Mittelpunkt stehen. Das Ziel dieser Aktivitäten besteht darin, die Teilnehmer bei der Entwicklung einer unternehmerischen Denkweise zu unterstützen.

Die letzten beiden Wochentage sind Einzel-Coachings gewidmet.Jeder Teilnehmer hat die Gelegenheit, mit dem Betreuer an der Entwicklung seiner Geschäftsidee zu arbeiten und Unterstützung bei der Erfüllung administrativer Auflagen für die Unternehmensgründung zu erhalten.

Zusätzlich zu den „Startrampen“ werden optionale Vorkehrungen für diejenigen getroffen, die sich für eine Unternehmensgründung interessieren. Diese umfassen einen Workshop zur Entwicklung eines Geschäftsmodells, Zugang zu Coworking-Räumen am Nachmittag, Besuche bei kommunalen und privaten Unternehmer- und Innovationszentren (z. B. das Eutokia-Zentrum in Bilbao) und diverse mit dem Unternehmertum zusammenhängende Veranstaltungen, wie etwa die „Tage des Unternehmertums“, die einmal im Jahr in den meisten spanischen Städten ausgerichtet werden.

Jede „Startrampe“ funktioniert als teilautonome Einrichtung. Jede von ihnen trägt die Verantwortung für dieVerwaltung ihrer eigenen Projekte, die Aufrechterhaltung, die Teilnehmerauswahl und die Einsetzung von mit bestimmten Funktionen betrauten Ausschüssen. Überwacht werden die „Startrampen“ durch das Programmleitungsteam, das die „Startrampen“ initiiert, für jede von ihnen eine Anfangsfinanzierung beschafft, die Methodik für die Kurse bereitstellt, die Ausbilder und Betreuer schult, spezielle Curricula entwickelt, Unterstützung bei der Verwaltung und pädagogischen Fragen leistet, die Qualität der erbrachten Dienstleistungen überwacht, Netzwerkveranstaltungen und wöchentliche Skype-Treffen für Betreuer organisiert, um den Austausch bewährter Verfahren und Innovationen zu erleichtern, und die Marke des Programms zur Verfügung stellt. Diese klare Rollendefinition bewirkt Skaleneffekte und verringert die Kosten des Systems.

Die Koordination der durch die Teilnehmer durchgeführten Arbeiten fällt unter die Verantwortung eines professionellen Betreuers. Dieser ist im Rahmen des Projekts in Vollzeit angestellt. Betreuer müssen die folgenden Kriterien erfüllen:

- Hochschulabschluss in den Sozialwissenschaften;

- Erfahrungen mit der Teamleitung;

- IKT-Fähigkeiten und digitale Kompetenzen; sowie

- ausgezeichnete Kommunikationsfähigkeiten.

Die ausgewählten Betreuer erhalten insgesamt 140 Schulungsstunden zu den Themen CoachingTechniken, Gruppendynamik, Personalmanagement, Unternehmertum, Führungsfähigkeiten, persönliches Branding und Entwicklung von Beschäftigungsfähigkeit. 40 Stunden dieser Schulungen finden als Präsenzveranstaltungen und 100 Stunden als Online-Schulungskurse statt.

\section{Projektfinanzierung}

Jede „Startrampe“ kostet etwa 40000 EUR, wobei die jeweiligen Kosten abhängig vom Umfang und der Dauer der geleisteten Unterstützung (d. h. fünf oder neun Monate) geringfügig abweichen. Die Kosten des Programms werden in Tabelle 19.1 dargestellt. 
Tabelle 19.1. Kosten pro Startrampe

\begin{tabular}{|c|c|c|}
\hline \multirow{2}{*}{ Ausgabe } & \multicolumn{2}{|c|}{ Betrag in EUR } \\
\hline & Minimum & Maximum \\
\hline 1. Betreuergehalt & 20000 & 20000 \\
\hline 2. Technische Kosten & 11000 & 12000 \\
\hline 3. Materialien & 4500 & 5000 \\
\hline 4. Reisekosten & 2000 & 2500 \\
\hline Gesamt & 37500 & 39500 \\
\hline
\end{tabular}

Den größten Ausgabeposten macht das Betreuergehalt aus. Für „Startrampen“ mit einer Laufzeit von sechs Monaten muss der Betreuer für mindestens acht Monate eingestellt werden, damit er angemessen geschult werden, das Programm vorbereiten und eine nachbereitende Evaluierung durchführen kann. Die technischen Kosten umfassen die Ausgaben für die Schulung und Unterstützung des Betreuers, die Gestaltung und Durchführung der Überwachung und die Nachbereitung mit den Kandidaten. Zu den Materialien gehören Bücher, Moderationskarten, die Kommunikation und eine Webseite. Reisekosten entstehen bei Besuchen bei Firmen oder Unternehmern, speziellen Veranstaltungen und Netzwerkveranstaltungen mit anderen „Startrampen“.

In das Programm ist eine breite Palette an Interessenträgern eingebunden, und es können einige Ressourcen durch Sacheinlagen gesichert werden. So werden zum Beispiel die Räumlichkeiten für die Programme in der Regel von den Stadtverwaltungen gestellt, und einige Betriebskosten werden von lokalen Firmen oder Stiftungen übernommen. Viele der Gastredner sind Freiwillige, und private Unternehmen bieten Mentoring für die Teilnehmer an. Die lokalen Hochschulen tragen zur Entwicklung von Indikatoren für die Wirkungsmessung bei.

Das Programm erhält Mittel aus verschiedenen Quellen, unter anderem:

- Die Regionalregierung von Kantabrien, die im Jahr 2013 vier Pilot-„Startrampen“ in Santander, Astilleros, Castro Urdiales und Torrelavega finanziert hat. Diese Investition betrug 240000 EUR.

- Die Stiftung Profesor Uría, die mit der spanischen Anwaltskanzlei Uría y Menéndez assoziiert ist (www.fundacionprofesoruria.org/), finanzierte 2014 gemeinsam mit der Stiftung Santa María die zweite „Startrampe“ in Aguilar de Campoo. Diese Investition betrug 33000 EUR.

- Die Barclays Bank stellte etwa 70\% der Mittel bereit, die für die Einrichtung und die Durchführung von drei „Startrampen“ (zwei in Madrid und eine weitere in Barcelona) benötigt wurden. Die "Startrampe" in Barcelona wird von Acción Contra el Hambre (www.accioncontraelhambre.org/ quienes_somos.php), einer der größten Nichtregierungsorganisationen Spaniens, geführt. Die Nichtregierungsorganisation stellte außerdem die übrigen 30\% der Mittel, die für die Durchführung des Programms benötigt wurden, über eine Finanzierung durch das operationelle Programm „Kampf gegen die Diskriminierung“ des ESF. Im Rahmen dieses Programms besteht der Beitrag des Programmteams hauptsächlich darin, die Methodik, technische Unterstützung, Auswahl und Schulung der Betreuer zu übertragen und diese drei „Startrampen“ in das größere „Startrampen“Netzwerk zu integrieren.

- Vor Kurzem hat der ESF dem Programm etwa 240000 EUR zur Verfügung gestellt - dies entspricht $80 \%$ des Betrags, der zur Finanzierung von acht neuen „Startrampen“ in der Region Andalusien im Jahr 2014 notwendig ist.

\section{Herausforderungen}

Eine der Herausforderungen für dieses Programm besteht darin, seine Auswirkungen zu bewerten und aufzuzeigen. Das Programmleitungsteam führt derzeit einen dreigliedrigen Ansatz zur Wirkungsmessung und Überwachung ein. Erstens verbessert das Team die Verfolgung der quantitativen Auswirkungen, d. h. der Anzahl der Begünstigten, die ein Unternehmen gründen oder einen festen 
Arbeitsplatz finden. Zweitens arbeitet es daran, die Messung der qualitativen Auswirkungen zu verbessern. Das Leitungsteam hat in Zusammenarbeit mit der Abteilung für Psychologie der Universidad Nacional de Educación a Distancia eine Forschungsumfrage entwickelt, mit der die „transformativen Auswirkungen“ des Programms auf seine Begünstigten bewertet werden sollen. Dazu gehören Verhaltensindikatoren wie Grad des persönlichen Antriebs, Gefühl der Befähigung, Kreativitätsniveau und Haltung gegenüber Unsicherheit, Rückschlägen oder neuen Möglichkeiten. Die Umfrage, die 2013 bei einer Stichprobe von Absolventen des Pilotprojekts „Startrampen“ validiert und vorab getestet wurde, verteilte man an die Teilnehmer der neuen „Startrampen“, die im September 2014 begannen. Ziel ist es, ihre Entwicklung im zeitlichen Verlauf zu verfolgen, indem die Umfrage zu Anfang des Programms, am Ende und sechs Monate nach Abschluss des Programms durchgeführt wird. Darüber hinaus wird im Rahmen des Programms ein Verfolgungssystem in Echtzeit erarbeitet. Eine mobile Anwendung wurde entwickelt, um Partnerorganisationen und/oder Kapitalgebern zu ermöglichen, die Entwicklung der „Startrampe(n)“, die sie unterstützen, in Echtzeit zu mitzuverfolgen. Die im Oktober 2014 entstandene Anwendung ermöglicht eine Überwachung der durchgeführten Aktivitäten (z. B. Unternehmerbesuche), von Ergebnissen (z. B. Konferenzen oder gemeinsam mit anderen Organisationen ausgerichtete Veranstaltungen) und von quantitativen Indikatoren.

Eine weitere Herausforderung für das Programm besteht darin, dass der Betreuer allein für die Bereitstellung der Unterstützung zuständig ist - andere Mitarbeiter gibt es nicht. Diese Aufgabe könnte für einen einzelnen Betreuer zu groß sein, und es besteht die Gefahr, dass der Betreuer sich verausgabt und nicht mehr in der Lage ist, seinen Pflichten nachzukommen. Angesichts des kleinen Haushalts versucht das Programmleitungsteam dieses Problems Herr zu werden, indem es Partnerschaften mit Hochschulen aufbaut, im Rahmen derer jeder „Startrampe“ ein Praktikant zur Verfügung gestellt wird. Dieser Praktikant würde den Betreuer bei seinen alltäglichen Aufgaben unterstützen.

\section{Auswirkungen}

Eine Abschätzung der Auswirkungen der Pilotphasen (April 2013 bis Dezember 2013) dauert an und umfasst fünf „Startrampen“. Laut Überwachungsdaten gründeten von den 114 Arbeitslosen (die Betreuer ausgenommen), die an der Pilotphase teilnahmen, $25 \%$ ein eigenes Unternehmen und $63 \%$ fanden einen festen Arbeitsplatz. Die übrigen $22 \%$ suchten entweder immer noch eine Beschäftigung oder hatten sich zu einer Anmeldung bei einer formellen Ausbildung entschlossen, um ihre Fähigkeiten und Kenntnisse zu verbessern.

Ende 2014 liefen im Rahmen des Programms insgesamt 40 „Startrampen“ mit 800 Teilnehmern. Wenn derselbe Anteil an Teilnehmern ein eigenes Unternehmen gründet (25\%), würde dies dazu führen, dass etwa 200 Arbeitslose Unternehmer würden. Man rechnet mit einer Verdoppelung der Anzahl der „Startrampen“ bis Ende 2015.

\section{Voraussetzungen für die Übertragung}

Eine der Stärken dieses Programms ist seine schnelle und kosteneffektive Skalierbarkeit. Zu den zentralen Voraussetzungen für eine wirksame Übertragung auf andere Kontexte zählen folgende:

1. Eine Philosophie der Befähigung. Das Programm betrachtet Arbeitslose als wertvolles Humankapital statt als Bürde oder Belastung für die Gesellschaft. Das pädagogische Modell des Programms betont die Bedeutung der Ausbildung der Menschen, damit sie die Fähigkeit entwickeln, sich selbst zu helfen. Die Teilnehmer werden nicht als Opfer einer Arbeitslosigkeitskrise angesehen, sondern als aktive Teilnehmer, die ihr eigenes Schicksal gestalten. Die Kernentscheidungen über die Konzeption des Modells gründen sich auf der Vorstellung der Befähigung als Mechanismus zur Einbeziehung, Selbstorganisation als Struktur und Unternehmertum als geistige Haltung.

2. Entwicklung einer starken Netzwerkstruktur. Das Organisationsmodell des Programms folgt einem kooperativen Gesellschaftsmodell, bei dem die Teilnehmer sich unter der Leitung des Betreuers selbst organisieren und zusammenarbeiten, um die Aktivitäten der „Startrampen“ in Eigenregie durchzuführen. Das Motto „Einer für alle und alle für einen“ erhöht das Engagement der Teilnehmer, verleiht ihnen ein Gefühl der Verantwortung und schafft eine starke Gemeinschaft. Außerdem 
steigert die Mitwirkung ganz unterschiedlicher Interessenträger, die sich ehrenamtlich einbringen, die Kostenwirksamkeit des Programms.

3. Einsatz eines klaren Betriebsmodells. Das Programm nutzt ein Modell von teilautonomen „Startrampen“, die von einer zentralen Einheit überwacht werden. Die klare Definition der Rollen der „Startrampen“ auf der einen und des Programmleitungsteams auf der anderen Seite sorgt für Skaleneffekte und verringert die Kosten des Systems. Allerdings brachte dieser flexible Ansatz zuweilen auch Probleme mit sich. Es muss sichergestellt werden, dass beide Parteien den Ansatz akzeptieren. In einigen Fällen entschieden die lokalen „Startrampen“ sich für einen autonomen Betrieb. Infolgedessen konnten die Teilnehmer nicht an anderen Programmveranstaltungen teilnehmen, und die Ergebnisse waren schwächer als in anderen „Startrampen“.

4. Auswahl der Teilnehmer. Da die Befähigung der Teilnehmer und ihre Fähigkeit zur Selbstorganisation von großer Wichtigkeit sind, ist eine geeignete Auswahl von Teilnehmern entscheidend. Ein Mindestmaß an Kompetenzen und persönlichem Antrieb sollte als Zugangsvoraussetzung festgelegt werden. Mehr als 90\% der Teilnehmer an dem Pilotprojekt 2013 hatten einen Hochschulabschluss oder eine abgeschlossene Berufsausbildung. Dies ist ein Indikator für Fähigkeiten und das Vermögen, sich einem Projekt zu verschreiben.

5. Nutzung von Partnerschaften mit Experten. Das Programm baut auf den Stärken der Stiftung Santa María la Real auf, die über mehr als 28 Jahre Erfahrung mit der Ausrichtung von Berufsbildungsworkshops mit benachteiligten Menschen und Arbeitslosen verfügt. Zudem hat die Stiftung ein sehr starkes partnerschaftliches Netzwerk aus öffentlichen und privaten Institutionen. Die Chancen für eine erfolgreiche Übertragung des Programms auf einen anderen Kontext dürften durch die Beteiligung einer solchen Organisation erhöht werden. Die Zusammenarbeit mit einer starken Organisation verleiht dem Projekt Glaubwürdigkeit, sowohl in den Augen potenzieller Teilnehmer als auch in denen potenzieller Partner (z. B. öffentliche Institutionen, Kapitalgeber).

\section{Literatur}

Eurostat (2015), Beschäftigung und Arbeitslosigkeit (AKE), verfügbar unter: http://ec.europa.eu/eurostat/ web/lfs/data/main-tables

LEES (2014), LEES-Website, verfügbar unter: www.lanzaderasdeempleo.es.

Resumen I Edición Del Programa „Lanzaderas de Empleo y Emprendimiento Solidario- LEES“, Fundación Santa María la Real, April 2014. 


\section{Botschafterinnen für Frauen, Schweden}

Diese Fallstudie stellt einen Ansatz vor, der Frauen dazu inspirieren soll, eine Unternehmensgründung und eine selbstständige Erwerbstätigkeit in Betracht zu ziehen. Die Ziele des Projekts und die Gründe für seine Schaffung werden beschrieben. Die Fallstudie beschreibt, wie das Projekt funktioniert, und stellt Daten vor, die seine Auswirkungen belegen. Neben den bei der Umsetzung des Projektes auftretenden Herausforderungen werden die Voraussetzungen für seine Übertragung auf einen anderen Kontext erörtert.

\section{Ziele}

Während der 1990er-Jahre starteten in Schweden mehrere kleine und mittlere Initiativen zur Förderung des weiblichen Unternehmertums. Die meisten dieser Initiativen wurden von der nationalen Regierung finanziert und koordiniert, die häufig Mittel und Schulungen für potenzielle Unternehmer und Unternehmensberater bereitstellte, die in den lokalen Kommunen tätig waren. Als die Regierung 2007 beschloss, politischen Initiativen zur Förderung des weiblichen Unternehmertums über einen Zeitraum von drei Jahren jährlich 100 Millionen SEK (etwa 10 Millionen EUR) zuzuteilen, erfuhren diese Initiativen eine Umgestaltung. Das Botschafterinnen-Programm wurde als zentrale Komponente dieses integrierten Ansatzes eingerichtet.

Das Programm setzt Unternehmerinnen als Rollenvorbilder ein, die ihre Erfahrungen teilen. Das Ziel besteht darin,

- die Sichtbarkeit des weiblichen Unternehmertums zu steigern;

- durch persönliche Geschichten und Rollenvorbilder zum weiblichen Unternehmertum zu inspirieren und es zu fördern;

- es den Frauen leichter zu machen, sich mit unternehmerischen Rollenvorbildern zu identifizieren;

- mehr Frauen dazu zu ermutigen, das Unternehmertum als potenzielle berufliche Laufbahn zu betrachten; und

- Frauen durch den Erfahrungsaustausch bei der Bewältigung ihrer unternehmerischen Herausforderungen zu helfen.

Die primäre Zielgruppe waren Schülerinnen an Mittelschulen, Studentinnen, Frauen in der Berufsausbildung, Karriereberaterinnen und Frauen mit Migrationshintergrund.

\section{Hintergrund}

Die Hauptmotivation hinter dem Projekt bestand darin, die Verhaltensnormen im Zusammenhang mit der Berufswahl zu beeinflussen, indem das allgemeine Bild des „Unternehmers“ als Tätigkeit für Männer hinterfragt wurde. Als die Öffentlichkeit in einer nationalen Umfrage gebeten wurde, einen 
Unternehmensinhaber oder Unternehmer zu benennen, gaben nur 6\% eine Frau an, obwohl 30\% der schwedischen Unternehmen von Frauen geleitet werden.

Um dieser Wahrnehmung entgegenzuwirken, wurden 880 Unternehmerinnen als Botschafterinnen ausgewählt, die zur Förderung des weiblichen Unternehmertums und zur Erhöhung der Attraktivität und Realisierbarkeit des Unternehmertums unter Frauen beitragen sollten. Die wahrgenommene Attraktivität ist „der Grad, zu dem man die Aussicht einer Unternehmensgründung anziehend findet, und spiegelt im Wesentlichen die eigenen Gefühle gegenüber dem Unternehmertum wider" (Krueger, 1993). Sie hängt von den Werten des Einzelnen ab, die wiederum aus seinem sozialen und kulturellen Umfeld herrühren (Shapero und Sokol, 1982). Tendiert das soziale und kulturelle Umfeld dazu, das Unternehmertum als „männlichen“ Beruf darzustellen, kann die wahrgenommene Attraktivität dieser Berufswahl unter den Frauen niedriger ausfallen (Bruni et al., 2004). Die wahrgenommene Realisierbarkeit hingegen steht für den Grad, zu dem sich jemand zu einer Unternehmensgründung fähig fühlt (Krueger et al., 2000). Sie rührt von dem Wissen und der Ausbildung des Einzelnen her sowie von der Verfügbarkeit von Rollenvorbildern, mit denen er sich identifizieren kann (Wilson et al., 2007).

Politische Initiativen wie das Botschafterinnen-Projekt zielen darauf ab, sowohl direkt - durch die Bereitstellung und Verbreitung von Rollenvorbildern, die die wahrgenommene Realisierbarkeit unter den Frauen beeinflussen („Ich kann das auch!“) - als auch indirekt auf Menschen einzuwirken, indem die wahrgenommene Attraktivität des Unternehmertums als Berufswahl für Frauen im Allgemeinen („Das ist ein guter und angemessener Beruf“) beeinflusst wird.

\section{Aktivitäten}

Das Botschafterinnen-Projekt wurde 2008 ins Leben gerufen und ursprünglich bis 2010 verlängert. Daraufhin wurde es für den Zeitraum 2011-2014 verlängert. Ende Dezember 2014 wurde das Projekt abgeschlossen.

Die schwedische Agentur für wirtschaftliches und regionales Wachstum war für die Koordination und Umsetzung des Projekts auf nationaler Ebene verantwortlich. Regionale Koordinatoren konnten Mittel beantragen, um das Botschafterinnen-Projekt in ihrer jeweiligen Region zu koordinieren. Die meisten der regionalen Koordinatoren kamen aus Unternehmens- oder Arbeitgeberorganisationen und brachten somit Erfahrungen aus der Leitung ähnlicher Projekte mit. Die regionalen Koordinatoren arbeiteten eng mit der schwedischen Agentur für wirtschaftliches und regionales Wachstum zusammen, um die politische Kohärenz und korrekte Umsetzung sicherzustellen. Beispielsweise waren die regionalen Koordinatoren für die Ausrichtung von Treffen und Möglichkeiten zur Netzwerkarbeit für die Botschafterinnen in ihrer Region zuständig und informierten die lokalen Behörden und Interessengruppen über das Projekt.

Zentral für den Erfolg des Programms war die Auswahl der Botschafterinnen, die durch ein Bewerbungsverfahren auf regionaler und nationaler Ebene ermittelt wurden. Für das Bewerbungsverfahren wurdein öffentlichen Radiosendungen, online und durch zahlreicheverschiedene Nichtregierungsorganisationen geworben. Interessierte Unternehmerinnen konnten sich online bewerben. Bei der Bewertung der Bewerbungen als Botschafterin wurde vor allem darauf geachtet, dass die zustande kommende Gruppe in Bezug auf Ethnizität, Alter, Branche, Unternehmensgröße und geografische Region vielfältig war. Frauen, die seit mehr als zwei Jahren ein Unternehmen führten, wurden bevorzugt. Die schwedische Agentur für regionales und wirtschaftliches Wachstum stellte Nachforschungen über die von den potenziellen Botschafterinnen geführten Unternehmen an, um sicherzustellen, dass diese noch aktiv waren.

Die Aufgabe der Botschafterinnen bestand darin, ihre eigene Geschichte zu erzählen und zu berichten, mit welchen Herausforderungen sie konfrontiert waren, wie sie sie bewältigt haben und warum sie gern als Unternehmerin tätig sind. Beispiele für die Aktivitäten der Botschafterinnen sind Vorträge an Schulen, der Erfahrungsaustausch mit Frauen, die an einer Unternehmensgründung interessiert sind, und die Entwicklung von Netzwerken für Geschäftsfrauen. Seit 2008 haben die Botschafterinnen im Rahmen des Projekts rund 170000 Menschen getroffen und waren an etwa 11000 Aktivitäten beteiligt. 
Eine weitere wichtige Funktion der Botschafterinnen ist die allgemeinere Förderung des weiblichen Unternehmertums, indem sie Informationen und Statistiken über das Unternehmertum von Frauen sichtbarer machen. Ziel der schwedischen Agentur für wirtschaftliches und regionales Wachstum ist es, „die Öffentlichkeit zu inspirieren“, gleichzeitig aber auch „ein realistisches Bild der Unternehmensführung zu vermitteln“. Indem Wissen über die Auswirkungen von Unternehmen in Frauenhand verbreitet wird, soll hervorgehoben werden, wie wichtig es ist, mehr Frauen für das Unternehmertum zu gewinnen.

Außerdem funktionieren einige der Botschafterinnen als Resonanzboden für andere Frauen, die ein eigenes Unternehmen gründen. Auf dieses Weise stellt das Botschafterinnen-Programm Frauen, die eine Unternehmensgründung anstreben, eine unmittelbare Beratung und Coaching zur Verfügung. Ab und zu geben die Botschafterinnen interessierten Frauen zudem Gelegenheit, ihnen bei der alltäglichen Arbeit zu folgen, um praktische Erfahrungen im Alltag einer Unternehmensinhaberin zu sammeln. Das Projekt bringt damit die Bemühungen um die Förderung des Unternehmertums und den Erfahrungsaustausch mit potenziellen Unternehmerinnen unter einen Hut.

Es verfügte über eine eigene Website, die verwendet werden konnte, um Treffen mit der Botschafterin $\mathrm{zu}$ vereinbaren und öffentlich Informationen $\mathrm{zu}$ verbreiten. Außerdem wurde ein Podcast erstellt, für den einige Botschafterinnen interviewt wurden.

Die Gruppe der Botschafterinnen bestand stets aus rund 800 Frauen, und über die Gesamtlaufzeit des Projekts betrachtet waren etwa 2000 Frauen als Botschafterinnen tätig. Einige dieser Frauen wurden auch zu Botschafterinnen der Europäischen Union ernannt und nahmen an Workshops und Treffen mit Botschafterinnen aus ähnlichen Programmen aus der gesamten Europäischen Union teil. So haben zum Beispiel Deutschland und Schottland ähnliche politische Initiativen ins Leben gerufen.

Das Botschafterinnen-Projekt war Teil eines größer angelegten Programms zur Förderung des weiblichen Unternehmertums. Das Gesamtprogramm erhielt während seiner beiden Phasen von 2007 bis 2010 und von 2011 bis 2014 eine staatliche Unterstützung von etwa 600 Millionen SEK, von denen 29 Millionen SEK (etwa 2,9 Millionen EUR) anfänglich in das Botschafterinnen-Projekt investiert wurden. Das Geld wurde für die Umsetzung und Koordination des Projekts auf nationaler und regionaler Ebene verwendet, indem Treffen für die Botschafterinnen arrangiert, Informationskampagnen durchgeführt und Evaluierungen vorgenommen wurden.

\section{Herausforderungen}

Das Botschafterinnen-Projekt stützte sich in hohem Maße auf freiwillige Botschafterinnen, um das Unternehmertum unter Frauen zu fördern. Zwar hielt das die Betriebskosten gering, doch bestand die Herausforderung darin, für ein anhaltendes Engagement unter den Botschafterinnen zu sorgen, insbesondere im ländlichen Raum, wo sie im Rahmen ihrer Aufgaben viel reisen mussten, um an Veranstaltungen teilzunehmen. Zum Beispiel musste eine Botschafterin unter Umständen mehrere Stunden zu einer Veranstaltung reisen, um dort eine kurze Präsentation zu halten. Auf die Botschafterinnen kommen also zuweilen hohe Opportunitätskosten zu. Dieses Problem wurde sowohl von den Botschafterinnen als auch von den regionalen Koordinatoren angesprochen.

Außerdem erwies sich die Auswahl der Botschafterinnen als schwierig. Ein Evaluierungsbericht übte Kritik an dem Verfahren der Selbstselektion, das zur Festlegung von Botschafterinnen eingesetzt wurde, denn einige der Botschafterinnen verfügten nicht über die nötigen Erfahrungen oder Fähigkeiten, um als Rollenvorbilder aufzutreten. In vielen Fällen beschäftigten die Unternehmerinnen keine Mitarbeiter in ihrem Unternehmen. Botschafterinnen mit großen, wachstumsstarken Unternehmen wären als inspirierende Rollenvorbilder unter Umständen besser geeignet gewesen. Die regionalen Koordinatoren erkannten diesen Umstand an, konnten aber keine Gründe vorbringen, um potenzielle Botschafterinnen abzulehnen, weil das Programm nicht mit einem ScreeningMechanismus ausgestattet ist.

Auch die Auswirkungen und die Kostenwirksamkeit des Programms werden zuweilen hinterfragt. Die grundsätzliche Frage lautet, ob „weiche“ politische Maßnahmen mit dem Unternehmertum zusammenhängende Verhaltensnormen beeinflussen können. Während staatliche Interventionen zur Förderung von Verhaltensnormen in Bezug auf die Gesundheit, Verbrauchersicherheit und 
Umweltfragen an Akzeptanz gewonnen haben (Thaler und Sunstein, 2008), sind der Frage, wie der Staat auf das wirtschaftliche Verhalten seiner Bürger, etwa in Bezug auf das Unternehmertum, Einfluss nehmen könnte und sollte, offensichtlich soziopolitische und ethische Grenzen gesetzt.

Darüber hinaus hat der schwedische Rechnungshof angemerkt, dass auf soziale Zielgruppen (z. B. Frauen und Zuwanderer) ausgerichtete Inspirations- und Mentoring-Programme zwar von ihren Teilnehmern geschätzt werden, in der Tendenz jedoch insgesamt eine sehr kleine Wirkung haben. Dies ist auch der internationale Eindruck, insbesondere in Bezug auf die Einflussnahme auf allgemeinere kulturelle Probleme, die die von Frauen wahrgenommene Attraktivität des Unternehmertums beeinträchtigen (z. B. Marlow und Patton, 2005).

\section{Auswirkungen}

Vom Start des Programms (Mai 2008) bis zu seinem Abschluss (Dezember 2014) erreichten die Botschafterinnen des Programms mehr als 170000 Menschen mit etwa 11000 Aktivitäten. Für jede Erfahrung wurden mittels eines Fragebogens die Ansichten der Teilnehmerinnen und Botschafterinnen erfasst.

Im Rahmen der Befragung sollten die Teilnehmerinnen die Botschafterinnen auf einer Skala von 1 bis 5 hinsichtlich des Inhalts und der Ausführung ihrer Präsentation bewerten. Insgesamt kommentierten $70 \%$ der Zuhörerinnen den Inhalt und die Ausführung der Präsentation mit „befriedigend“, „gut“ oder „sehr gut“. Die Teilnehmerinnen wurden zudem zu ihrem „Interesse am Unternehmertum“ befragt, und 50\% derjenigen, die einer Botschafterin zugehört hatten, bekundeten ein „größeres Interesse“ am Unternehmertum nach dem Treffen mit der Botschafterin.

Im Rahmen ihrer Selbstbewertung der in dem Projekt gesammelten Erfahrungen betrachteten $50 \%$ der Botschafterinnen ihre Rolle als hilfreich für sich als Unternehmerinnen. $\mathrm{Zu}$ den als hilfreich betrachteten Faktoren zählten eine stärkere persönliche Marke, ein erweitertes soziales Netzwerk und verbesserte Kompetenzen. Bei ihrer Bewertung der Programmverwaltung waren 70\% der Botschafterinnen „zufrieden“ oder „sehr zufrieden“ mit der Koordination des Programms auf nationaler und regionaler Ebene.

Im Jahr 2013 wurden besondere Anstrengungen unternommen, unter Lehrern an weiterführenden Schulen für das Programm zu werben, da Sekundarschülerinnen eine der primären Zielgruppen des Programms waren. Umfragedaten zufolge kannten 55\% der Lehrer, die mit Betriebswirtschaft und Unternehmertum für Sekundarschüler zu tun hatten, das Botschafterinnen-Programm.

Laut Statistiken der schwedischen Agentur für Wachstumspolitik und -analyse verzeichnet die Projekt-Website (www.ambassadorer.se) seit ihrem Bestehen rund 800 individuelle Besucher wöchentlich. Das Programm war während seiner Laufzeit etwa 200-mal jährlich in den Medien zu sehen.

\section{Voraussetzungen für die Übertragung}

Im Jahr 2009 rief die Europäische Union ein europäisches Netzwerk für Botschafterinnen für das weibliche Unternehmertum ins Leben, das durch das schwedische Programm und andere Pioniere wie das Vereinigte Königreich inspiriert wurde. ( $\left.{ }^{6}\right)$ Das Botschafterinnen-Konzept wird inzwischen in 21 anderen Ländern der Europäischen Union umgesetzt. Die wichtigsten Erfolgsfaktoren für ein solches Programm können wie folgt definiert werden:

1. Aufbau eines Pools engagierter Botschafterinnen. Das Engagement und die Bereitschaft der Botschafterinnen, einen freiwilligen Beitrag zu leisten, sind für eine erfolgreiche Umsetzung von wesentlicher Bedeutung. Allerdings ist auch ein Auswahlverfahren wichtig, um sicherzustellen, dass die Botschafterinnen qualifiziert sind.

6 Weitere Informationen erhalten Sie unter: http://ec.europa.eu/growth/smes/promoting-entrepreneurship/we-work-for/ women/support-networks/index_en.htm. 
2. Sicherstellung, dass die Botschafterinnen repräsentativ sind. Da sich die Verhaltensnormen verschiedener Bevölkerungsgruppen und Untergruppen unterscheiden, müssen die Botschafterinnen in Bezug auf ihr Alter, ihre Geschäftserfahrung, ihren kulturellen Hintergrund usw. vielfältig vertreten sein, um eine glaubwürdige Wirkung auf die Zuhörer (sowohl Frauen als auch Männer) zu erzielen.

3. Intensive Werbemaßnahmen. Es müssen unbedingt Mechanismen zur Kontaktaufnahme wie Websites, Social Media usw. genutzt werden, um Bürger, insbesondere die jungen unter ihnen, zu erreichen.

4. Übermittlung positiver Botschaften. Die Aktivitäten der Botschafterinnen sollten so weit wie möglich so gestaltet sein, dass sie das Potenzial haben, die in der Theorie ermittelten Mechanismen der wahrgenommenen Attraktivität („Das ist eine angemessene und potenziell attraktive Berufswahl“) und der wahrgenommenen Realisierbarkeit („Ich kann das auch“) unter den Teilnehmern zu bewirken.

5. Bewertung der Auswirkungen. Den Evaluierungen sollten verschiedene Indikatoren zugrunde gelegt werden, unter anderem die Selbstbewertung der Erfahrungen durch die Botschafterinnen, die Auswirkungen auf die Teilnehmer und sekundärstatistische Angaben wie die Medienberichterstattung. Die Evaluierungen sollten wie im schwedischen Beispiel durch unabhängige Parteien vorgenommen werden.

\section{Literatur}

Bruni, A., S. Gherardi und B. Poggio (2004), „Entrepreneur-mentality, gender and the study of women entrepreneurs", Journal of Organizational Change Management, Bd. 17, S. 256-268.

Krueger, N. (1993), „The Impact of Prior Entrepreneurial Exposure on Perceptions of New Venture Feasibility and Desirability“, Entrepreneurship: Theory and Practice, Bd. 18, S. 5-21.

Krueger, N., M. D. Reilly und A.L. Carsrud (2000), „Competing models of entrepreneurial intentions“, Journal of Business Venturing, Bd. 15, S. 411-432.

Marlow, S. und D. Patton (2005), „All credit to men? Entrepreneurship, finance, and gender“, Entrepreneurship Theory and Practice, Bd. 29, S. 717-735.

Shapero, A. und L. Sokol (1982), „The Social Dimensions of Entrepreneurship“ in Kent, C. A. und D. L. Sexton und K. E. Vesper (Hrsg.), The Encyclopaedia of Entrepreneurship, Englewood Cliffs: Prentice-Hall, S. 72-90.

Thaler, R. H. und C. R. Sunstein (2008), Nudge, Yale University Press.

Wilson, F., J. Kickul und D. Marlino (2007), „Gender, entrepreneurial self-efficacy, and entrepreneurial career intentions: Implications for entrepreneurship education“, Entrepreneurship Theory and Practice, Bd. 31, S. 387-406. 


\section{Access to Work, Vereinigtes Königreich}

Diese Fallstudie stellt ein Beispiel dafür vor, wie Menschen mit Behinderungen bei der Unternehmensgründung mit Beihilfen aus einem allgemeinen Unterstützungsprogramm unterstützt werden können. Die Ziele des Programms werden erörtert und die Gründe für die Bereitstellung dieser Unterstützung vorgestellt. Die Fallstudie beschreibt, wie die Unterstützung funktioniert und wie man Zugang zu ihr erhält. Zudem werden Daten zu den erreichten Ergebnissen vorgestellt. Neben den größten bei der Bereitstellung dieser Unterstützung auftretenden Herausforderungen werden die Voraussetzungen für die Übertragung dieses Ansatzes auf einen anderen Kontext vorgestellt.

\section{Ziele}

Access to Work ist ein Programm im Vereinigten Königreich, dessen Zielgruppe Menschen sind, deren Gesundheitszustand oder Behinderung sich auf ihre Erwerbsfähigkeit auswirkt. Es richtet sich an Menschen mit chronischen gesundheitlichen Problemen oder Einschränkungen, die eine zusätzliche praktische Unterstützung benötigen, um erwerbstätig zu werden oder zu bleiben. Ziel des Programms ist die Verringerung von Ungleichheiten am Arbeitsplatz zwischen Menschen mit und Menschen ohne Behinderungen, indem verschiedene Hindernisse beseitigt werden.

Es läuft seit 1994 im gesamten Vereinigten Königreich (einige seiner Bestandteile sind jedoch schon viel länger vorhanden) und ist noch heute in England, Schottland und Wales verfügbar. Es gewährt Beihilfen, um Personen (oder Arbeitgebern) bei der Bezahlung einer Unterstützung oder Ausstattung zu helfen, die notwendig ist, um eine Stelle anzutreten oder zu behalten oder um ein Unternehmen zu gründen. Die Beihilfen können von Angestellten, selbstständig Erwerbstätigen oder von Menschen, die eine Unternehmensgründung anstreben, in Anspruch genommen werden. Diejenigen, die sich für eine Unternehmensgründung interessieren, können Access to Work in Kombination mit der New Enterprise Allowance (Zuwendung für neue Unternehmen) nutzen, die 26 Wochen lang eine wöchentliche Zuwendung auszahlt, um den Unternehmer während der Frühphase der Unternehmensentwicklung zu unterstützen. Die Höhe der von Access to Work gewährten Beihilfe hängt von den jeweiligen Umständen ab. Sie muss nicht zurückgezahlt werden und hat keinen Einfluss auf andere Leistungen, die Empfänger eventuell beziehen. ${ }^{7}$

\section{Hintergrund}

Die Selbstständigenquoten unter den Menschen mit Behinderungen sind häufig höher als unter den Menschen ohne Behinderung, da eine selbstständige Erwerbstätigkeit die nötige Flexibilität erlauben kann, mit der Behinderung zurechtzukommen, während man gleichzeitig auf

7 Bezieht der Antragsteller jedoch bestimmte andere Leistungen, kommt er unter Umständen nicht für eine gleichzeitige Unterstützung durch Access to Work infrage. 
dem Arbeitsmarkt aktiv bleibt (z. B. Meager und Higgins, 2011; Pagán, 2009). Dies unterstreicht das wachsende Arbeitsmarktpotenzial von Menschen mit Behinderungen, denen eine selbstständige Erwerbstätigkeit ermöglicht wird. Die Absicht von Access to Work ist es, Aktivitäten auf dem Arbeitsmarkt (d. h. sowohl die abhängige als auch die selbstständige Erwerbstätigkeit) für Menschen mit Behinderungen zu fördern. Das Programm unterstützt außerdem die selbstständige Erwerbstätigkeit und Unternehmensgründungen durch formelle Verbindungen zur New Enterprise Allowance.

Das Grundprinzip hinter Access to Work lautet, dass Menschen mit Behinderungen eventuell ihren Arbeitsplatz (z. B. die Ausstattung) oder ihre Arbeitsregelungen (z. B. die Arbeitszeiten) anpassen müssen, um das Ausmaß ihrer Benachteiligung im Rahmen ihrer Erwerbstätigkeit zu minimieren. Abhängig von der Art der Behinderung sind mögliche Anpassungen unter anderem Schulungsmöglichkeiten, der Kauf neuer Geräte (z. B. eine spezielle Tastatur bei Arthritis), die Vornahme physischer Veränderungen am Arbeitsplatz (z. B. der Einbau einer Rampe für Rollstuhlfahrer) oder die Verbesserung der Arbeitsplatzregelungen (z. B. ein eigener Schreibtisch statt zeitversetzt gemeinsam genutzte Schreibtische). Access to Work finanziert kosteneffektive Lösungen für Angestellte und selbstständig Erwerbstätige.

Dieses Programm steht auch im Einklang mit allgemeinen sozialpolitischen Maßnahmen, die dafür sorgen sollen, dass Bevölkerungsgruppen, die von sozialer Ausgrenzung bedroht sind, aktiv bleiben. Das Armutsrisiko in der Europäischen Union ist bei Menschen mit Behinderungen erheblich höher als bei Menschen ohne Behinderungen (21,1\% gegenüber 14,9\%), was vor allem durch die niedrigen Beschäftigungsquoten der Menschen mit Behinderungen verursacht wird (Hauben et al., 2012).

\section{Aktivitäten}

Access to Work wird von Jobcentre Plus durchgeführt. Das Programm bietet Beratung und praktische Unterstützung für Menschen mit Behinderungen und ihre Arbeitgeber, um ihnen dabei zu helfen, Arbeitshindernisse zu bewältigen, die durch eine Behinderung verursacht werden. Neben Beratung und Informationen für Menschen mit Behinderungen und Arbeitgeber zahlt das Programm über Jobcentre Plus eine Beihilfe aus, um die Kosten für „angemessene Anpassungen“ von Arbeitsplätzen zu decken. Diese leisten einen Beitrag zur Beseitigung der mit der Behinderung zusammenhängenden Herausforderung für Angestellte oder selbstständig Erwerbstätige (das Accessto-Work-Programm). Konkret kann das Programm folgende Zuzahlungen leisten (Dewson et al., 2009):

- Beihilfen für spezielle Hilfsmittel und Ausstattung, die Menschen mit Behinderungen bei der Bewältigung von mit ihrer Behinderung zusammenhängenden Hindernissen am Arbeitsplatz helfen.

- Beihilfen für Anpassungen an Räumlichkeiten und Ausstattung, die einen Teil der Kosten für die Zugänglichmachung von Räumlichkeiten und Ausstattung decken.

- Beihilfen für den Arbeitsweg, die zur Verfügung stehen, um Zusatzkosten für den Arbeitsweg oder Wege innerhalb des Arbeitsplatzes für Menschen zu decken, die keine öffentlichen Verkehrsmittel nutzen können.

- Beihilfen für Betreuer, die dem Antragsteller erlauben, die Dienste eines Betreuers in Anspruch zu nehmen. Beispiele für die Betreuung am Arbeitsplatz sind etwa das Vorlesen für sehbehinderte Menschen, Kommunikation durch Gebärdensprache für Hörbehinderte (außer beim Vorstellungsgespräch - diese Kosten werden durch die Beihilfe zur Kommunikationsunterstützung bei Vorstellungsgesprächen gedeckt), die Bereitstellung eines speziellen Coachings für Menschen mit Lernschwierigkeiten oder Hilfe bei der Körperpflege.

- Beihilfen für verschiedene Ausgaben, um mit einer Behinderung verbundene Hindernisse zu beseitigen, die nirgendwo sonst erfasst sind, z. B. Parkhauskosten, weil der Betroffene wegen seiner Behinderung einen bequemen, aber kostenpflichtigen Platz in einem Parkhaus benötigt.

- Beihilfen zur Kommunikationsunterstützung bei Vorstellungsgesprächen, die die Kosten für den Einsatz eines Dolmetschers vollständig decken, um die Kommunikationsbarrieren bei Vorstellungsgesprächen zu beseitigen. 
Jegliche Unterstützung durch Access to Work gilt als zusätzlich zu den angemessenen Anpassungen durch Arbeitgeber gemäß dem Disability Discrimination Act (Gesetz zu Diskriminierungen aufgrund einer Behinderung). Um für Access to Work infrage zu kommen, müssen die Antragsteller die Definition eines Menschen mit Behinderung gemäß dem Disability Discrimination Act erfüllen oder eine Behinderung haben, die sie am Arbeitsplatz beeinträchtigt (Thornton und Gordon, 2002; Dewson et al., 2009). Das Programm steht Menschen offen, die mindestens 16 Jahre alt sind, eine Behinderung haben, in England, Schottland oder Wales wohnhaft sind und in Kürze eine Stelle oder eine Probearbeit antreten werden oder abhängig oder selbstständig erwerbstätig sind. Meistens werden zwar Unternehmen unterstützt, die Menschen mit Behinderungen beschäftigen, doch auch selbstständig Erwerbstätigen und Unternehmensgründern steht diese Unterstützung zur Verfügung. Access to Work bietet Beihilfen für einzelne Menschen mit Behinderungen oder ihre Arbeitgeber zur Erstattung genehmigter Kosten und geht flexibel auf individuelle Bedürfnisse ein. Die Beihilfe wird jedoch nicht für ehrenamtliche Tätigkeiten geleistet.

Die Behinderung oder gesundheitlichen Probleme müssen die Erwerbsfähigkeit des Antragstellers beeinträchtigen oder bedeuten, dass der Antragsteller arbeitsbezogene Kosten zu tragen hat. Bezieht der Antragsteller andere Leistungen, beispielsweise Invaliditätsunterstützung, Zuschuss für eingeschränkt Erwerbsfähige, Schwerbehindertengeld, Einkommensunterstützung und anrechenbare Zeiten zur Rentenversicherung, kommt er unter Umständen nicht für eine gleichzeitige Unterstützung durch Access to Work infrage. Die Beihilfe von Access to Work kann bei einem Stellenwechsel auch übertragen werden.

Selbstständig Erwerbstätige und Unternehmensgründer werden von Access to Work insbesondere durch Spezialausstattung, Betreuer und bei den Reisekosten unterstützt. Ziel ist es, dass Menschen mit Behinderungen sich genau wie alle anderen Menschen für eine Unternehmensgründung entscheiden können. Access to Work strebt an, die Zahl der selbstständig erwerbstätigen Menschen mit Behinderungen weiter zu erhöhen. Diese liegt bei etwa 500000 Menschen bzw. 15\% der Menschen mit Behinderungen im erwerbsfähigen Alter. Im Vergleich dazu sind 3,2 Millionen Menschen ohne Behinderung selbstständig erwerbstätig.

Für die Beihilfe von Access to Work ist kein bestimmter Betrag vorgegeben - die Höhe hängt von den individuellen Umständen ab (z. B. Dauer der Erwerbstätigkeit, Unternehmensgröße des Arbeitgebers, Unterstützungsbedarf und selbstständig erwerbstätig oder nicht). Die genaue Kostenaufteilung wird zwischen dem Arbeitgeber (oder dem selbstständig Erwerbstätigen) und dem Programmberater vereinbart. Bei selbstständig Erwerbstätigen und Unternehmensgründungen kann die Beihilfe zur Erstattung von Unterstützungskosten bis zu $100 \%$ der genehmigten Kosten betragen (Access to Work, 2014).

Berater im Bereich Beschäftigung von Menschen mit Behinderungen in den lokalen Zweigstellen des Jobcentre Plus bieten Rat bei der Beantragung einer Beihilfe des Programms. Der Antragsteller kann sich außerdem an die regionalen Kontaktzentren von Access to Work wenden, um herauszufinden, ob er für eine Beihilfe infrage kommt. Operative Unterstützungseinheiten übernehmen die Verwaltung von Access to Work (Dewson et al., 2009).

\section{Projektfinanzierung}

Der Haushalt von Access to Work wird innerhalb der Ausgabengrenzen des Ministeriums für Beschäftigung und Renten verwaltet. Der Haushalt ist von 14,6 Millionen GBP (etwa 21,9 Millionen EUR) in den Jahren 1997-1998 auf 69 Millionen GBP (etwa 78,3 Millionen EUR) in den Jahren 20082009 gestiegen und auf diesem Niveau geblieben. (Access to Work, 2014).

\section{Herausforderungen}

Access to Work leidet darunter, dass es unter seinen potenziell Begünstigten recht wenig bekannt ist. Es wird keine umfassende Werbung für das Programm gemacht, und viele Begünstigte erfuhren durch einen glücklichen Zufall oder „versehentlich“ davon, meist durch inoffizielle Quellen oder von Behindertenorganisationen. Größere und öffentliche Organisationen und Wohltätigkeitsorganisationen wussten mit größerer Wahrscheinlichkeit von dem Programm als kleine Unternehmen und 
selbstständig Erwerbstätige. Darüber hinaus deuten Evaluierungen darauf hin, dass das schriftliche und auf der Website befindliche Werbematerial verbessert werden könnte, indem anhand von echten Fallbeispielen mehr Einzelheiten zu den Arten der verfügbaren Unterstützung und zur Zielgruppe aufgenommen werden. Außerdem wurden Bedenken über den Informationsfluss zwischen Access to Work und anderen Sachbearbeitern von Jobcentre Plus sowie anderen Fachleuten (z. B. medizinischen Fachkräften) geäußert. Laut diesen Ergebnissen muss diesen wichtigen Kommunikationskanälen Priorität eingeräumt werden, damit Mitarbeiter, die in Kontakt mit den Klienten kommen, diese an das Programm verweisen können.

Wenngleich die meisten Begünstigten des Programms sich positiv zum Antragsverfahren äußerten, haben sie einige Bedenken aufgeworfen (Dewson et al., 2009):

- Schwierigkeiten, den Sachbearbeitern die Art ihrer Behinderung(en) oder ihres Beschäftigungsstatus zu erklären oder verständlich zu machen. Dies ist besonders für selbstständig Erwerbstätige schwierig;

- die Antragsformulare sind etwas unflexibel und nicht in alternativen Formaten verfügbar. Menschen mit Lernschwierigkeiten hatten am meisten Probleme mit den bürokratischen Verfahren des Programms;

- Verzögerungen beim Abschluss des Antragsverfahrens und der Bereitstellung der Unterstützung.

Im Allgemeinen hatten die Klienten mit mehreren Behinderungen bei dem Verfahren mit den größten Herausforderungen zu kämpfen. Dasselbe gilt für das Bewertungsverfahren. Unzufriedenheit mit dem Bewertungsverfahren kam zwar relativ selten vor, wurde jedoch von denjenigen vorgebracht, deren Unterstützungsbedarf komplex oder vielfältig war. Außerdem wurden in der Evaluierung mitunter Bedenken in Bezug auf die Objektivität der Bewertung sowie über die Einheitlichkeit der Entscheidungen geäußert, die von den verschiedenen Sachbearbeitern und in den verschiedenen Regionen gefällt wurden. Die Schlüsselbotschaft lautete demzufolge, dass die Kommunikation zwischen allen am Bewertungs- und Entscheidungsfindungsverfahren Beteiligten verbessert werden muss, um sicherzustellen, dass beim Vorschlag von Lösungen den Bedürfnissen der Kunden in vollem Umfang Rechnung getragen wird. Und schließlich berichteten einige Nutzer über Schwierigkeiten, wenn ihr Unterstützungsbedarf aufgrund von Veränderungen ihrer Umstände (z. B. wegen einer Verschlechterung ihres Gesundheitszustands, eines Stellenwechsels oder weil sie bei der Beibehaltung und Aufrechterhaltung ihrer Unterstützung durch Access to Work Hilfe benötigten) überprüft werden musste. Hier wurde wiederum eine intensivere Kommunikation zwischen den Sachbearbeitern und Kunden sowie Nachbetreuungsdienste als Lösung vorgeschlagen (Dewson et al., 2009).

Bei den selbstständig Erwerbstätigen zeigen sich diese Herausforderungen noch deutlicher. Für Jobcentre Plus ist es eine anspruchsvolle Aufgabe, kleine Unternehmen und insbesondere die selbstständig Erwerbstätigen zu erreichen. Dies geschieht häufig über Vermittler wie Buchhalter, Rechtsanwälte und Handelskammern. Die Mitarbeiter von Jobcentre Plus sind geschult worden und haben mehr Informationen über das Programm erhalten, um es besser bei kleinen Unternehmen und selbstständig Erwerbstätigen vermarkten zu können (Access to Work, 2014).

\section{Auswirkungen}

Im Jahr 2012 half Access to Work mehr als 30000 Menschen mit Behinderungen, Arbeitsplätze zu finden oder beizubehalten. Von diesen Menschen waren 4500 in Kleinunternehmen tätig. Untersuchungen zeigen außerdem, dass $45 \%$ der Klienten arbeitslos wären, hätten sie keine Unterstützung durch das Programm erhalten (Ministerium für Arbeit und Renten, 2013). Auf der Grundlage der Evaluierungen gibt es klare Belege dafür, dass die Art der von dem Programm geleisteten Unterstützung einige positive Beschäftigungsergebnisse herbeiführt.

Die Betreuerbeihilfen waren wesentlich, um einen Arbeitsplatz zu finden und eine Beschäftigung aufrechtzuerhalten. Die Evaluierung deutet ferner darauf hin, dass ein Betreuer für selbstständig Erwerbstätige von zentraler Wichtigkeit sein könnte, um ein Unternehmen auf die Art und Weise zu führen, die einem Menschen ohne Behinderung offen steht. Der Beistand eines Betreuers zeigte Kollegen wie Kunden, dass Menschen mit Behinderungen gleichberechtigt arbeiten können, und 
trug zum Abbau von Vorurteilen bei. Außerdem brachte der Einsatz eines Betreuers erhebliche gesundheitliche Vorteile, die den Unterschied zwischen der Aufrechterhaltung der Erwerbstätigkeit und dem Aufgeben ausmachten. Allerdings nahmen die Empfänger diese Unterstützung seltener in Anspruch als andere Unterstützungsleistungen wie Beihilfen für spezielle Hilfsmittel und Ausstattung (Dewson et al., 2009).

Trotz des Potenzials von Access to Work zur Förderung von selbstständig Erwerbstätigen ist die Inanspruchnahme relativ gering, weshalb sich die Evaluierungen auch nicht auf diese Klienten konzentrieren. Im Jahr 2012 gründeten etwa 8000 Klienten von Access to Work ein Unternehmen mittels der New Enterprise Allowance (Ministerium für Arbeit und Renten, 2013). Beispiele für die gegründeten Unternehmen sind unter anderem ein mobiler Hundesalon, eine Edelschokoladenfirma und ein Tapas-Restaurant.

Die Klienten sind im Allgemeinen mit der Unterstützung, die sie erhalten, zufrieden (Thornton und Corden, 2002; Dewson et al. 2009). Klienten mit weniger komplexen Bedürfnissen, z. B. einer Hilfe bei den Kosten für den Arbeitsweg, waren häufiger zufrieden, während diejenigen mit komplexeren Bedürfnissen häufig ein gewisses Maß an Unzufriedenheit mit dem Access-to-Work-Programm äußerten (Dewson et al., 2009). Das Antragsverfahren wurde überwiegend als zügig, bedarfsgerecht und professionell empfunden, und die Antragsteller berichteten, dass sie während des Verfahrens ein hohes Maß an Unterstützung erhielten, zum Beispiel telefonisch. In ähnlicher Weise wurde das Bewertungsverfahren als gründlich und umfassend betrachtet. Die Gutachter wurden überwiegend als Experten in ihrem Bereich angesehen, und die Kunden waren beeindruckt, wie detailliert und professionell die Leistungen erbracht wurden. Viele Begünstigte berichteten, sie hätten viel von den Begutachtern gelernt, sowohl in Bezug auf die gesundheitlichen Probleme oder Behinderungen, mit denen sie konfrontiert waren, als auch hinsichtlich der verfügbaren Lösungen (Dewson et al., 2009).

Bei der Bewertung der Auswirkungen von Access to Work müssen unbedingt die Kosten aufgrund von Mitnahmeeffekten berücksichtigt werden. Die Evaluierungen zeigen, dass die verschiedenen Unterstützungsarten des Programms unterschiedliche Verluste aufgrund von Mitnahmeeffekten mit sich bringen. Die Beihilfe für den Arbeitsweg ist ein Beispiel für eine fast vollständige Zusätzlichkeit, d. h., die geleistete Unterstützung wäre andernfalls, etwa durch den Arbeitgeber, nicht geleistet worden. Teilweise Mitnahmeeffekte sind relativ üblich bei Access to Work, weil die Unterstützung für Menschen mit Behinderungen verfügbar gewesen wäre, jedoch länger gedauert hätte oder nicht so umfassend gewesen wäre wie die Unterstützung, die Access to Work leistet. Im Access-to-WorkProgramm wurden keine Fälle von vollständigen Mitnahmeeffekten berichtet, was darauf hindeutet, dass für die Klienten immer ein gewisses Maß an Mehrwert entsteht (Dewson et al., 2009). Insgesamt gilt das Programm als kosteneffektiv und erzielte für das Finanzministerium eine klare Kapitalrendite: 1,48 GBP für jedes britische Pfund, das für das Programm ausgegeben wurde (Ministerium für Arbeit und Renten, 2013; Access to Work, 2014).

\section{Voraussetzungen für die Übertragung}

Die folgenden zentralen Erfolgsfaktoren sind wahrscheinlich wichtig bei dem Versuch, einen ähnlichen Ansatz zur Unterstützung von Menschen mit Behinderungen bei der Aufnahme einer selbstständigen Erwerbstätigkeit umzusetzen:

1. Sicherung von erheblichen Finanzmitteln. Zusätzlich zur Finanzierung der verschiedenen Bestandteile des Programms müssen die Kontaktarbeit und die Anpassung der Antragsformulare für die unterschiedlichen Behinderungen finanziert werden.

2. Umfassende Kommunikation und Nutzung anderer Unterstützungsprogramme, um Bekanntheit zu erzielen. Auf der Grundlage der Evaluierungen scheint es, dass Access to Work nicht in vollem Umfang von Menschen mit Behinderungen und von Arbeitgebern genutzt wurde, und dass das nicht ausgeschöpfte Potenzial bei den selbstständig Erwerbstätigen und kleinen Unternehmen noch größer ist. Im Allgemeinen ist der Grund für die geringe Durchdringung des Programms seine bescheidene und lückenhafte Bekanntheit aufgrund unzureichenden Marketings und unzureichender Kommunikation unter den Interessenträgern. 
3. Vorauszahlungen. Kleine Unternehmen und (potenzielle) Unternehmer stehen vor der Gewährung der Beihilfe in Bezug auf die Bezahlung von Ausstattung, Betreuern und Reisekosten womöglich vor Liquiditätsproblemen. Um dem Unterstützungsbedarf dieser besonderen Zielgruppe durch dieses (oder ein ähnliches) Programm zu entsprechen, muss das Erstattungsverfahren neu überdacht werden.

4. Kombination mit anderen Unterstützungsleistungen für selbstständig Erwerbstätige. Diese Erfahrung hat gezeigt, dass es nützlich sein kann, Beihilfen für Ausstattung, Betreuer und Reisen mit anderen Programmen zu kombinieren (z. B. der New Enterprise Allowance), um Unternehmensgründungen bei Menschen mit Behinderungen zu fördern.

5. Schulung der Programmmitarbeiter. Es wurde berichtet, dass die Programmmitarbeiter Schwierigkeiten hatten, die verschiedenen Formen von Behinderungen $\mathrm{zu}$ verstehen. Dies erschwerte vielen Antragstellern, insbesondere denen mit mehrfachen Behinderungen, das Antrags- und Evaluierungsverfahren. Die Bereitstellung von Sensibilitätsschulungen für die an vorderster Linie tätigen Mitarbeiter kann dazu beitragen, diese Schwierigkeiten zu bewältigen.

\section{Literatur}

Access to Work (2014), „The Access to Work Scheme“, verfügbar unter: www.publications.parliament.uk/ pa/cm200809/cmselect/cmworpen/158/15807.htm (Zugriff am 27. Juni 2014).

Access to Work (2014b), Website von Access to Work, verfügbar unter: www.gov.uk/access-to-work, (Zugriff am 3. Juni 2014).

Ministerium für Arbeit und Renten und The Rt Hon Esther McVey (2013), „New Year - new start up support for disabled entrepreneurs", www.gov.uk/government/news/new-year-new-start-up-supportfor-disabled-entrepreneurs (Zugriff am 27. Juni 2014).

Dewson, S., D. Hill, N. Meager und R. Willison (2009), „Evaluation of Access to Work: Core Evaluation. Ministerium für Arbeit und Renten“, Forschungsbericht Nr. 619.

Hauben, H., M. Coucheir, J. Spooren, D. McAnaney und C. Delfosse (2012), „Assessing the impact of European governments' austerity plans on the rights of people with disabilities“, in European Foundation Centre (Hrsg.), Europäischer Bericht, S. 19-24.

Meager, N. und T. Higgins (2011), „Disability and Skills in a Changing Economy, UK Commission for Employment and Skills“, Briefing Paper Series, verfügbar unter: www.oph.fi/download/140962_ equality-disability.pdf

New Enterprise Allowance (2014), Website der New Enterprise Allowance, verfügbar unter: www.gov.uk/new-enterprise-allowance (Zugriff am 3. Juni 2014).

Pagán, R. (2009), „Self-employment among people with disabilities: Evidence for Europe“, Disability and Society, Bd. 24, Nr. 2, S. 217-229.

Thornton, P. und A. Corden (2002), „Evaluating the impact of Access to Work: A case study approach“, Social Policy Research Unit, The University of York. 


\section{Prowess, Vereinigtes Königreich}

Diese Fallstudie stellt ein Beispiel vor, wie Organisationen zur Unternehmensförderung akkreditiert werden können, um sicherzustellen, dass sie eine hochwertige Unterstützung leisten. Die Ziele dieses Ansatzes sowie sein Hintergrund werden beschrieben und seine Funktionsweise erläutert. Zur Veranschaulichung der Auswirkungen dieses Projekts werden Daten präsentiert. Ferner erörtert die Fallstudie die Herausforderungen bei der Durchführung dieser Art von Maßnahme und die Voraussetzungen für ihre Übertragung auf einen anderen Kontext.

\section{Ziele}

Bei Prowess handelt es sich um ein Mitgliedschaftsnetzwerk aus Organisationen, die eine frauenfreundliche Unterstützung für die Unternehmensgründung im gesamten Vereinigten Königreich leisten. Es wurde 2002 mit Unterstützung der Regierung als National Association for the Promotion of Women's Enterprise (NationalerVerband zur Förderung des weiblichen Unternehmertums), eine Gesellschaft mit beschränkter Nachschusspflicht, im Vereinigten Königreich gegründet und war in dieser Form bis 2011 tätig. Prowess wurde damit beauftragt, die erste nationale Strategie für weibliches Unternehmertum und Qualitätsstandards für die Unterstützung frauengeführter Unternehmen zu erarbeiten. In seiner Hochphase hatte Prowess mehr als 350 Mitgliedsorganisationen, die insgesamt über 100000 weibliche Unternehmensinhaber unterstützten.

Außerdem wirkte Prowess bei der Lobbyarbeit bei der Regierung für die Einrichtung eines Ausschusses und einer Arbeitsgruppe für frauengeführte Unternehmen mit. Dies trug dazu bei, dass in einer Zeit häufiger politischer Führungswechsel im Regierungsministerium für Unternehmen das weibliche Unternehmertum im Fokus der Politik blieb. Dabei wurde auch eine Reihe von Schulungsmaterialien, Berichte über bewährte Verfahren und Qualitätsstandards für Dienstleistungen zur Unternehmensförderung von Unternehmerinnen erstellt. Der bekannteste Qualitätsstandard war der Flagship Award, der die Qualität der Unternehmensförderung aus einer „frauenfreundlichen“ Perspektive messen soll.

Die Gesellschaft Prowess wurde 2012 aufgrund von Kürzungen im Staatshaushalt geschlossen, doch der ehemalige Geschäftsführer erwarb ihre Vermögenswerte und legte Prowess anschließend als Sozialunternehmen neu auf. Es hat sich seither mit einem stärkeren Fokus auf die Unterstützung durch privatwirtschaftliche Akteure neu erfunden. So hat es zum Beispiel die Charta für Frauen in der Geschäftswelt entwickelt. Dabei handelt es sich um einen Verhaltenskodex und ein Schulungs- und Marketinginstrument für Organisationen, die Frauen dabei unterstützen möchten, erfolgreiche Unternehmen zu gründen, aufrechtzuerhalten und wachsen zu lassen. Auf diese Weise funktioniert es nach wie vor als Netzwerk, das lokale Anbieter einer frauenfreundlichen Unternehmensförderung verbindet. 


\section{Hintergrund}

Anfang der 2000er-Jahre waren im Vereinigten Königreich die Unternehmerinnen für etwa ein Drittel der Unternehmensgründungen verantwortlich. Sie waren unter den neuen Unternehmensinhabern klar unterrepräsentiert. Obwohl sich das im Laufe des letzten Jahrzehnts deutlich gewandelt hat - Frauen sind nun für mehr als 50\% der neuen Unternehmensgründungen verantwortlich -, sind frauengeführte Unternehmen in der Tendenz unterkapitalisiert und agieren in einem recht eingeschränkten Bereich von Sektoren mit niedriger Wertschöpfung (Eurostat, 2015).

Anfang der 2000er-Jahre erkannte der Small Business Service (Dienst für Kleinunternehmen), dass Dienstleistungen zur Unternehmensförderung nicht genügend Frauen und andere soziale Zielgruppen (z. B. junge Menschen, Zuwanderer, Menschen mit Behinderungen) erreichten. Den Dienstleistungen zur Unternehmensförderung gelang es häufig nicht, Frauen zur Aufnahme einer selbstständigen Erwerbstätigkeit zu ermutigen, und sie erkannten das wirtschaftliche Potenzial der Frauen nicht.

Prowess wurde als Netzwerk von Organisationen zur Unternehmensförderung für das weibliche Unternehmertum eingerichtet. Das Programm wurde während eines Zeitraums ins Leben gerufen, als die Regierung eine Politik verfolgte, gemäß der spezialisierte Agenturen zur Unterstützung bestimmter Zielgruppen entwickelt werden sollten. Die Frauen bildeten die größte dieser Zielgruppen. In dieser Zeit entstanden eine Reihe lokaler und nationaler Agenturen. Einige von ihnen sind zwar heute noch tätig, darunter die Wirtschaftsförderungsagentur für Frauen, doch viele der Aktivitäten wurden entweder wieder in allgemeine Programme und Agenturen eingegliedert, zurückgefahren oder endeten. Dennoch existiert immer noch eine bedeutende Gruppe von Organisationen zur Unternehmensförderung, die am Austausch von Materialien und bewährten Verfahren für die Unterstützung des weiblichen Unternehmertums interessiert sind.

\section{Aktivitäten}

Auch wenn Prowess an vielfältigen Aktivitäten mitwirkt, unter anderem Fürsprache und Lobbyarbeit auf nationaler und regionaler Ebene sowie Bereitstellung von Unterstützungsdiensten für die Mitgliedsorganisationen (z. B. jährliche Veranstaltungen), war einer seiner größten Beiträge der Prowess Flagship Award. Mit dieser Auszeichnung sollten frauenfreundliche Dienstleistungen zur Unternehmensförderung als Teil eines über den Phoenix Entwicklungsfonds finanzierten Projekts anerkannt werden. Dabei handelt es sich um einen nationalen Fonds, der sich auf die Unternehmensförderung in benachteiligten Gebieten und in Gruppen, die im Unternehmertum unterrepräsentiert sind, konzentriert.

Der Flagship Award ist eine Auszeichnung auf der Grundlage von zwölf Kriterien, anhand deren allgemeine und spezialisierte Anbieter einer Unternehmensförderung für Frauen bewertet werden können. Die Geschäftsleitung von Prowess legte die Kriterien fest und versuchte darin die bewährten Verfahren für die Förderung des weiblichen Unternehmertums festzuhalten. Die zwölf Standards für Unternehmensförderungsanbieter decken die vier Schlüsselbereiche Einbeziehung, Gleichstellung, Klientenfokus und Qualität ab (Tabelle 22.1).

Prowess führte Bewertungen aller seiner Mitgliedsorganisationen durch. Zu jeder Bewertung gehörte ein Standortbesuch, bei dem ein kleines Team von Prowess sich mit der Organisation und einigen ihrer Klienten traf, um die Aktivitäten der Organisation anhand der zwölf Kriterien zu bewerten.

Von den 68 Organisationen, die in der ersten Runde bewertet wurden, erhielten sechs den Status „Flagship-Mitglied“. Ferner handelte es sich bei neun Mitgliedsorganisationen um Netzwerke, die anhand von leicht unterschiedlichen Kriterien bewertet wurden. Zwei Mitgliedern wurde der Status „FlagshipNetzwerkmitglied“ verliehen. Aktuell gibt es 24 Organisationen, die einen Flagship Award erhalten haben.

Im Jahr 2011 wurde der Flagship Award unter Prowess 2.0 eingestellt und durch die Charta für Frauen in der Geschäftswelt ersetzt. Dabei handelt es sich um einen Verhaltenskodex und ein Marketing- und Schulungsinstrument für Anbieter von Dienstleistungen zur Unternehmensförderung und Dienstleistungen, die Frauen bei der Gründung eines Unternehmens und bei dessen Wachstum unterstützen. Die Charta baut auf den Prowess Flagship Awards und der Arbeit anderer führenden Initiativen in diesem Bereich (z. B. der Initiative für die Entwicklung kleiner Firmen, dem internationalen Standard für Unternehmerinnenzentren) auf, gehtjedoch noch weiter, indem sie zusätzlich zu einer optionalen Akkreditierung einen Verhaltenskodex für Mitarbeiter und Schulungen vorsieht. 


\section{Tabelle 22.1 Prowess-Flagship-Standards}

Standard

Der Anbieter erbringt regelmäßig Dienstleistungen durch persönliche Treffen.

2 Der Anbieter erbringt seit mindestens einem Jahr zielgerichtete Unterstützungsleistungen für Frauen.

3 Der Anbieter hilft mindestens 50 Unternehmensinhaberinnen pro Jahr (30 in abgelegenen Regionen)

4 a. Der Anbieter gliedert statistische Daten zu seinen Klienten nach Geschlecht, Ethnizität, Behinderung und sonstigen relevanten Merkmalen auf, durch die benachteiligte und unterrepräsentierte Gruppen ermittelt werden können, und der Anbieter erreicht eine angemessene Anzahl dieser Gesellschaftsgruppen.

b. Der Anbieter engagiert sich für vielfältige Gemeinschaften.

5 Der Anbieter ist kundenorientiert und versucht stets, seine Dienstleistungen zu verbessern und die Bedürfnisse der Klienten zu erfüllen. Er verfügt über Methoden, um sich die Erfahrungen und Bedenken der Klienten anzuhören.

6 Der Anbieter verfügt über ein aktives Kontaktaufnahmeprogramm (z B. Vorträge/Besuche an Orten, die Frauen frequentieren)

$7 \quad$ Mindestens ein Drittel des Fachpersonals des Unternehmensförderungsanbieters muss aus Frauen bestehen.

8 Der Anbieter bemüht sich aktiv darum, sicherzustellen, dass Frauen Zugang zu den Unterstützungsleistungen erhalten.

Mindestens $40 \%$ der Klienten sind Frauen.

10 Falls der Anbieter zur Bereitstellung eines kohärenten Dienstleistungsangebots mit Partnern zusammenarbeitet, hat er Einfluss auf diese und wird über deren Tätigkeiten informiert.

11 Der Anbieter gewährt direkt oder durch Partnerschaften Zugang zu den folgenden Unterstützungsleistungen für Frauen:

- Netzwerkarbeit

- Schulungen oder Beratung vor der Unternehmensgründung

- Unternehmerschulungen oder -beratung

- Hilfe beim Zugang zu Finanzierung (d. h. Beihilfen und/oder Darlehen für die Unternehmensgründung und -entwicklung, und/oder fundierte Orientierungshilfen zu geeigneten Finanzierungsquellen und Unternehmensberatung, um sicherzustellen, dass die Frauen auf eine Investition vorbereitet sind)

- IKT-Schulungen

12 Der Anbieter hilft Klienten dabei, für ihre Unternehmen zu werben und sie bei sich bietenden Gelegenheiten zu feiern (z. B. indem sie sie ermutigen, an Wettbewerben teilzunehmen, Mitgliederverzeichnisse zu erstellen oder sich um die Veröffentlichung von Fallstudien in der Presse zu bemühen).
Gründe

Fehlendes Selbstvertrauen stellt für viele Frauen, die ein Unternehmen gründen, ein Problem dar. In den Anfangsphasen sind persönliche Dienstleistungen wesentlich. Regelmäßige persönliche Dienstleistungen bauen zudem Vertrauen auf und tragen dazu bei, dass die Unternehmen wissen, dass sie die Dienste mitgestalten können.

Die Reflexion über Erfahrungen und Feedback der Klienten stellt einen wichtigen Teil des Prozesses zur Gestaltung eines hochwertigen Unterstützungsprogramms für frauengeführte Unternehmen dar.

Hochwertige Programme müssen einer effektiven Programmgestaltung und -durchführung verpflichtet sein. Dies ist wichtig, um die Zielklienten zu erreichen.

Um Marktsegmente wirksam und gezielt zu erreichen, ist es wichtig, die Klienten zu verstehen und zu wissen, wie sie erreicht werden können.

Zur Sicherstellung von nachhaltigen Dienstleistungen ist eine strategische Mission notwendig.

Organisationen, die bewährte Verfahren einsetzen, hören auf ihre Klienten und handeln nach deren Feedback. Es ist wichtig, dass Verfahren wie Sozialaudits oder Klientenbefragungen vorhanden sind.

Viele Frauen sehen sich nicht als „Geschäftsfrauen“, daher müssen das traditionelle Marketing und Kontaktaufnahmen sensibel auf den Klientenstamm ausgerichtet sein

Unterstützungsorganisationen müssen sich bemühen, die Merkmale ihrer Klienten widerzuspiegeln. Weibliche Fachkräfte können bei der Unternehmensförderung starke Rollenvorbilder für Klienten sein, und weibliche Klienten fühlen sich mit anderen Frauen zuweilen wohler.

Betreuungsaufgaben müssen berücksichtigt werden - Frauen mit Betreuungsaufgaben benötigen Zugang zu einer Kinderkrippe oder Unterstützung bei den Kinderbetreuungskosten ohne ein Übermaß an Einschränkungen. Ältere oder Unterhaltsberechtigte mit einem sonstigen Pflegebedarf stellen zunehmend ein Problem dar, denn Frauen übernehmen am ehesten die Hauptverantwortung für diese. Unbedingt zu bedenken ist auch die Frage der Kultur, denn in manchen Kulturen nehmen Frauen nicht an gemischtgeschlechtlichen Kursen teil.

Innerhalb des strategischen Rahmens für frauengeführte Unternehmen liegt die Zielquote der Regierung für Frauen, die staatlich finanzierte Dienstleistungen zur Unternehmensförderung in Anspruch nehmen, bei 40\%. Diese Zielvorgabe müssen allgemeine Unternehmensförderungsagenturen erreichen.

Beziehungen zu Partnern, an die Klienten weitervermittelt werden können, sind wichtig, doch Organisationen die bewährte Verfahren einsetzen, bemühen sich um engere Beziehungen zu Organisationen, die ihnen die Bereitstellung kohärenter Dienstleistungen ermöglichen. Außerdem legen sie dieselben hohen Maßstäbe an die Partnerorganisationen wie an sich selbst an.

Frauen sind häufig offener, wenn nur Frauen an Sitzungen beteiligt sind. Dies führt zu umfassenderen Diskussionen. Diese Sitzungen können Gelegenheit bieten, um Kontakte zu knüpfen, und dadurch die Frauen zu Netzwerken verbinden.

Diese Sitzungen stärken das Selbstvertrauen und die persönliche Effizienz (z. B. Zeitmanagement und Verhandlungsgeschick). Dies sind essenzielle Grundlagen für Menschen, die nach einer langen Arbeitslosigkeit ein Unternehmen gründen.

Unternehmen von Frauen sind unverhältnismäßig oft unterkapitalisiert. Unternehmensförderungsanbieter können Hilfestellung leisten, indem sie einen direkten Zugang zu Darlehen und Beihilfen oder zu Beratern ermöglichen, die die Situation von Unternehmerinnen verstehen.

Laut einigen Berichten machen Frauen in ihrem Unternehmen mit geringerer Wahrscheinlichkeit Gebrauch von IKTFähigkeiten. Unternehmerische IKT-Fähigkeiten sollten ein Kernbestandteil der Unternehmerschulungsprogramme sein, und Klienten sollten ermutigt werden, IKT-Fähigkeiten ggf. für Recherchen oder Werbemaßnahmen für ihr Unternehmen einzusetzen.

Viele Geschäfte werden über informelle und formelle Netzwerke durchgeführt - traditionell sind Frauen jedoch mit geringerer Wahrscheinlichkeit an Unternehmer- oder sonstigen Netzwerken beteiligt. Netzwerke, die ausschließlich aus Frauen bestehen, können ein Sprungbrett für die Beteiligung an verschiedenen Netzwerken sein und bieten selbst Möglichkeiten zur wichtigen gegenseitigen Unterstützung unter Frauen in derselben Lage.

Werbung hat den doppelten Vorteil, dass für das Unternehmen geworben wird (und für viele Frauen sind Vertrieb und Marketing ein besonderes Problem) und relevante Rollenvorbilder für andere Frauen, die eine Unternehmensgründung anstreben, geschaffen werden. 


\section{Projektfinanzierung}

Der Phoenix Entwicklungsfonds der Regierung des Vereinigten Königreichs und die Gemeinschaftsinitiative EQUAL der Europäischen Union unterstützten Prowess bis 2005. Im Anschluss wurde Prowess autark. Prowess erwirtschaftet derzeit jährlich etwa 450000 EUR mit Mitgliedsgebühren. Sonderveranstaltungen tragen weitere 150000 EUR bei. Eine weitere kleine Einnahmenquelle ist die Beratung.

\section{Herausforderungen}

Die größte Herausforderung, mit der Prowess konfrontiert war, bestand in der Sicherung der Finanzierung und einer stabilen Basis an Wirtschaftsförderungsorganisationen angesichts der sich ändernden Prioritäten der Regierung in Bezug auf die Unterstützung von Unternehmen. Sein ursprünglich wichtigster Partner war die staatliche Einrichtung Small Business Service (Dienst für Kleinunternehmen), der jedoch ab 2004 allmählich aufgelöst wurde.

2005 wurde die Organisation Business Link (der nationale, mit öffentlichen Mitteln finanzierte Unternehmensberatungs- und -betreuungsdienst) auf die neun englischen regionalen Entwicklungsagenturen übertragen. In der Folge gab es auf nationaler Ebene keine einzelne Einrichtung mehr, die für Unternehmen zuständig war. Aus diesem Grund entschloss Prowess, mit jeder der neun regionalen Entwicklungsagenturen über die Fortsetzung der Finanzierung zu verhandeln, um seine Tätigkeit aufrechtzuerhalten. Fünf der regionalen Entwicklungsagenturen, unter anderem die Entwicklungsagentur in den East Midlands, die die Bewegung der regionalen Entwicklungsagenturen für Frauen und Unternehmen anführte, setzten die Arbeit mit Prowess fort.

Die regionalen Entwicklungsagenturen wurden 2010 bei einem Regierungswechsel abgeschafft. Zwar wurden mehr als 30 lokale Unternehmenspartnerschaften in ganz England mit der Aufgabe der Unternehmensförderung betraut, doch verfügten diese nur über begrenzte Ressourcen, um Wirtschaftsförderungsdienste speziell für Frauen zu unterstützen. Im Vergleich zu der Lage im Jahr 2000, als der einheitliche Small Business Service und eine einzige Marke das Land abdeckten, hatte das allgemeine Unternehmensförderungssystem in England bis 2010 eine Fragmentierung erfahren. Prowess arbeitet noch heute mit diesen Strukturen. Die lokale Unternehmenspartnerschaft New Anglia hat eine Mitgliedschaft an der neuen Charta für Frauen in der Geschäftswelt erworben und wird seine Dienste 2014 mittels des Selbstbewertungstools überprüfen.

Prowess stand zudem vor einer Reihe finanzieller Probleme. Insbesondere das Ende der Finanzierung aus dem Phoenix Fonds im Jahr 2006 führte zu einer sofortigen Verringerung seiner Mittel. Außerdem wurden häufig spezialisierte Agenturen, die beim Flagship-Award-Programm eine zentrale Rolle spielten und oft zahlende Mitglieder von Prowess waren, im Rahmen der Vereinfachung und Konsolidierung der Dienstleistungen zur Unternehmensförderung abgewickelt. Prowess war auch vom Ende der Unterstützung durch die Gemeinschaftsinitiative EQUAL des Europäischen Sozialfonds (ESF) und der Schwierigkeit betroffen, eine Nachfolgefinanzierung aus dem allgemeinen ESF-Programm zu finden. Zudem war Prowess nach dem Zusammenbruch von Lehman Brothers von einer Verringerung der Unterstützung durch die Banken gefährdet. Mehrere Banken, u. a. RBS, NatWest und Lloyds TSB, hatten Prowess aus ihren Haushalten für soziale Unternehmensverantwortung finanziert.

\section{Auswirkungen}

Prowess wurde im Rahmen einer eingehenden Evaluierung des Phoenix Entwicklungsfonds als Fallstudie bewertet, die 2005 veröffentlicht wurde. Die Fallstudie validierte die Relevanz, die der Ansatz von Prowess dank der Flagship Awards besitzt.

Auch die Auswirkungen von Prowess in den East Midlands sind ein Beweis für die Errungenschaften des Programms. Die Aufnahme des weiblichen Unternehmertums wurde in der regionalen Entwicklungsstrategie für die East Midlands hervorgehoben. Das weibliche Unternehmertum wurde als eine der „Hauptauszeichnungen“ ausgewählt, die vom Gremium für spezielle Unterstützungsdienste festgelegt wurden (insgesamt waren es zehn Hauptauszeichnungen). Dies war auch eine Gelegenheit, um für Bekanntheit und zusätzliche finanzielle Unterstützung zu sorgen. 
Auch auf nationaler Ebene konnte Prowess Auswirkungen erzielen. Einer nachhaltigen Strategie folgend, um seine Dienstleistungen frauenfreundlicher zu gestalten (unter anderem durch den Flagship Award), erhöhte der nationale Dienst Business Link den Frauenanteil unter seinen Klienten zwischen 2003 und 2006 von einem Fünftel auf ein Drittel (Women's Enterprise Task Force, 2009). Prowess arbeitete eng mit dem Netzwerk Business Link zusammen, um ihm bei der Umsetzung dieser Strategie zu helfen, und mehreren Partnern von Business Link wurde ein Flagship Award verliehen.

Ein Teil des Vermächtnisses ihres Ansatzes lebt außerdem in den Bewertungskriterien der Initiative für die Entwicklung kleiner Firmen weiter, die zur Akkreditierung von Initiativen zur Unternehmensförderung genutzt werden. Die Kriterien beziehen sich darauf, wie gut die Organisation die Bedürfnisse diverser Klienten mit unterschiedlichem Hintergrund erfüllen können.

\section{Voraussetzungen für die Übertragung}

Auch wenn die Prowess Flagship Awards für frauenfreundliche Unternehmensförderung während ihres zehnjährigen Bestehens viel Lob ernteten, ist es bemerkenswert, dass aus anderen Teilen der Europäischen Union keine Entsprechungen bekannt sind. Im Prinzip gibt es nichts, was Ministerien auf nationaler oder regionaler Ebene oder Organisationen, die das weibliche Unternehmertum fördern möchten, daran hindern würde, diesen Ansatz zu übernehmen. Jedoch funktionieren solche Akkreditierungsprogramme am besten, wenn die nationale Politik starken Druck ausübt, um Dienste für frauengeführte Unternehmen zu verbessern. Dies ist wesentlich, wenn ein Akkreditierungsmarkt tragfähig sein soll. Für eine erfolgreiche Übertragung gelten wahrscheinlich die folgenden Voraussetzungen:

1. Ein starker Druck aus den Ministerien, die niedrigen Quoten weiblicher Unternehmen anzugehen. Dafür ist eine Forschungsgrundlage notwendig sowie die Anerkennung des Umstands, dass niedrigere Gründungsund Überlebensraten unter frauengeführten Unternehmen ein Problem für die Wirtschaft und die Gesellschaft darstellen. Außerdem müssen die politischen Entscheidungsträger erkennen, dass eine Verbesserung der Qualität der Unternehmensförderung notwendig ist.

2. Sicherstellung einer Mindestdauer, um Glaubwürdigkeit aufzubauen. Während der ersten Jahre ihrer Tätigkeit benötigen Organisationen wie Prowess öffentliche Unterstützung, um Instrumente wie den Flagship Award einzurichten und weiterzuentwickeln. Idealerweise sollte diese Unterstützung mindestens fünf Jahre zur Verfügung gestellt werden.

3. Es muss Nachfrage bestehen. Damit die Auszeichnungen Erfolg haben können, muss es Organisationen geben, die sich akkreditieren lassen möchten. Der Flagship Award war am besten für einen durch die öffentliche Hand finanzierten Unternehmensförderungsansatz geeignet, bei dem eine Reihe allgemeiner Unternehmensförderungsorganisationen und spezialisierter Anbieter, die Unternehmerinnen fördern, beteiligt sind (Gründungszentren, Unternehmenszentren, Unternehmensberatungs- und CoachingProgramme, finanzielle Unterstützung). Die anschließend entwickelte Charta eignet sich eher für ein privatwirtschaftliches Modell.

4. Unterstützung von Organisationen, die für Verbesserungen offen sind. Damit die Auszeichnung funktioniert, muss sie den Unternehmensförderungsorganisationen als positiver Prozess verkauft werden, in dem sie sich verbessern können. Dafür müssen die Unternehmensförderungsorganisationen bereit sein, sich anhand von objektiven Kriterien bewerten zu lassen, und die Kapitalgeber der Unternehmensförderung darauf bestehen, dass die Unterstützung auf frauenfreundliche Weise erbracht wird.

\section{Literatur}

Eurostat (2015), Beschäftigung und Arbeitslosigkeit (AKE), verfügbar unter: http://ec.europa.eu/eurostat/web/ lfs/data/main-tables

Women's Enterprise Task Force (2009), „Greater Return On Women's Enterprise (GROWE): The UK Women's Enterprise Task Force's Final Report and Recommendations“, verfügbar unter: https://pure.strath.ac.uk/ portal/files/17970755/D130_WETF_Growe_Report_2.4_1_.pdf. 

OECD PUBLISHING, 2, rue André-Pascal, 75775 PARIS CEDEX 16 (842016015P1) ISBN 978-92-6426138-9 - 2016 


\title{
Integrationsfördernde Unternehmensgründung KOMPENDIUM BEWÄHRTER VERFAHREN
}

\author{
Inhaltsverzeichnis \\ Zusammenfassung \\ Leseleitfaden
}

Teil I. Herausforderungen, Möglichkeiten und Erfolgsfaktoren von Politiken für integrationsfördernde Unternehmensgründungen

Kapitel 1: Zentrale politische Fragen und Ansätze für integrationsfördernde Unternehmensgründungen Kapitel 2: Bestandteile erfolgreicher Programme für integrationsfördernde Unternehmensgründungen

Teil II. Beispiele für bewährte Verfahren anhand von Programmen für ein integrationsförderndes Unternehmertum

Unternehmensgründungsprogramm, Österreich

DreamStart, Belgien (Brüssel)

Unternehmertum funktioniert, Belgien (Flandern)

Frauen auf dem Arbeitsmarkt, Kroatien

Geschäftscoaching für ethnische Minderheiten, Dänemark

Beihilfe zur Unternehmensgründung, Finnland

Adie Mikrofranchising, Frankreich

Neuer Gründungszuschuss, Deutschland

Kiútprogram, Ungarn

Going for Growth, Irland

Invitalias Unterstützung für das Unternehmertum Arbeitsloser, Italien

Massnahme zur Aufnahme einer gewerblichen Tätigkeit oder selbstständigen Erwerbstätigkeit, Lettland

Förderungsfonds für das Unternehmertum, Litauen

Sozialhilfe für selbstständig Erwerbstätige (BBZ), Niederlande

Reifer Unternehmer, Polen

Unternehmerisch in die Geschäftswelt, Slowenien

Startrampen für Beschäftigung und Unternehmertum, Spanien

Botschafterinnen für Frauen, Schweden

Access to Work, Vereinigtes Königreich

Prowess, Vereinigtes Königreich

Diese Veröffentlichung steht Ihnen online zur Verfügung unter http://dx.doi.org/10,1787/9789264261402-de.

Dieses Werk wird in der OECD iLibrary veröffentlicht, die eine Sammlung sämtlicher Bücher, Zeitschriften und statistischen Datenbanken der OECD enthält. Besuchen Sie www.oecd-ilibrary.org für weitere Informationen.

OECDpublishing www.oecd.org/publishing
ISBN 978-92-6426138-9

842016015 P1
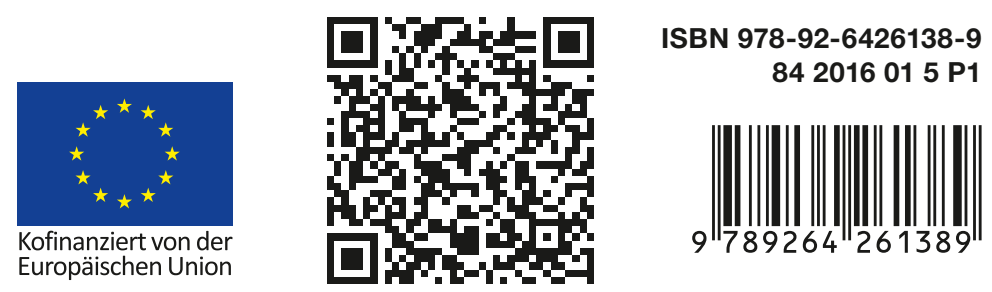